\title{
Light-Driven N-Heterocyclic Carbene Catalysis Using Alkylborates
}

\author{
Yukiya Sato, ${ }^{1}$ Yamato Goto ${ }^{1}$ Kei Nakamura, ${ }^{1}$ Yusuke Miyamoto, ${ }^{1}$ Yuto Sumida, ${ }^{1 *}$ \\ and Hirohisa Ohmiya ${ }^{1,2 *}$
}

${ }^{1}$ Division of Pharmaceutical Sciences, Graduate School of Medical Sciences, Kanazawa University, Kakuma-machi, Kanazawa 920-1192, Japan

2 JST, PRESTO, 4-1-8 Honcho, Kawaguchi, Saitama 332-0012, Japan

*E-mail: Yuto Sumida: sumida@p.kanazawa-u.ac.jp
Hirohisa Ohmiya: ohmiya@p.kanazawa-u.ac.jp

\section{Table of Contents}

$\begin{array}{ll}\text { 1. Instrumentation and Chemicals } & \mathrm{S} 2\end{array}$

2. General Procedure for Synthesis of Boracene-Alkylborates S5

3. General Procedure for Synthesis of PDP-Alkylborates S6

4. Detailed Optimization Conditions for Two-Component Coupling S7

5. General Procedure for Two-Component Coupling S9

6. Cyclic Voltammetry of Acyl Azolium Intermediates S10

7. UV/Vis specta of Reaction Substrates $\quad$ S11

8. Photo-quenching Experiment: Stern-Volmer Plot S13

$\begin{array}{ll}\text { 9. Stoichiometric Experiment } & \text { S14 }\end{array}$

$\begin{array}{ll}\text { 10. Characterization Data for Acylation Products } & \text { S21 }\end{array}$

$\begin{array}{ll}\text { 11. Optimization of Vicinal Alkylacylation of Alkenes } & \text { S28 }\end{array}$

12. General Procedure for Three-Component Coupling $\quad$ S29

13. Characterization Data for Alkylacylation Products $\quad$ S30

$\begin{array}{ll}\text { 14. References } & \text { S46 }\end{array}$

- NMR Spectra 


\section{Instrumentation and Chemicals}

NMR spectra were recorded on Bruker AVANCE NEO 400N spectrometer, operating at 400 $\mathrm{MHz}$ for ${ }^{1} \mathrm{H} \mathrm{NMR}, 61.4 \mathrm{MHz}$ for ${ }^{2} \mathrm{H} \mathrm{NMR}, 100.6 \mathrm{MHz}$ for ${ }^{13} \mathrm{C} \mathrm{NMR}, 376.5 \mathrm{MHz}$ for ${ }^{19} \mathrm{~F}$ NMR and 128.4 MHz for ${ }^{11} \mathrm{~B}$ NMR. Chemical shifts were reported in $\delta \mathrm{ppm}$. Chloroform- $d_{1}\left(\mathrm{CDCl}_{3}\right)$ containing $0.03 \%$ tetramethylsilane (TMS) $(>99.8 \% \mathrm{D}$, Cambridge Isotope Laboratories, Inc., Cat. No. DLM-7), acetone- $d_{6}(99.96 \% \mathrm{D}$, Cambridge Isotope Laboratories, Inc., Cat. No. DLM-38) and dimethyl sulfoxide- $d_{6}(99.9 \% \mathrm{D}$, Cambridge Isotope Laboratories, Inc., Cat. No. DLM-10) were used as solvents for NMR measurements at ambient temperature. Chemical shifts $(\delta)$ for ${ }^{1} \mathrm{H}$ NMR are given in parts per million (ppm) relative to relative to TMS $\left(\delta 0.00 \mathrm{ppm}\right.$ in $\left.\mathrm{CDCl}_{3}\right)$, or residual acetone $(\delta$ $2.07 \mathrm{ppm})$. Chemical shifts $(\delta)$ for ${ }^{13} \mathrm{C}$ NMR are given in ppm relative to $\mathrm{CDCl}_{3}(\delta 77.0 \mathrm{ppm})$, or residual acetone $(\delta 30.6 \mathrm{ppm})$ or residual dimethyl sulfoxide $(\delta 39.5 \mathrm{ppm})$. Chemical shifts $(\delta)$ for ${ }^{19} \mathrm{~F}$ NMR are given in ppm relative to $\alpha, \alpha, \alpha$-trifluorotoluene $\left(\delta-63.0 \mathrm{ppm}\right.$ in $\left.\mathrm{CDCl}_{3}\right)$ used as the external standard. Chemical shifts $(\delta)$ for ${ }^{11} \mathrm{~B}$ NMR are given in ppm relative to $\mathrm{BF}_{3} \cdot \mathrm{OEt}_{2}\left(\delta 0.0 \mathrm{ppm}\right.$ in $\left.\mathrm{CDCl}_{3}\right)$ used as the external standard. Chemical shifts $(\delta)$ for ${ }^{2} \mathrm{H}$ NMR are given in ppm relative to $\mathrm{CHCl}_{3}(\delta$ $7.26 \mathrm{ppm}$ ). The abbreviations s, d, t, q, br s, and m signify singlet, doublet, triplet, quartet, heptet, broad singlet, and multiplet, respectively.

DART-Mass (HRMS) were measured with JMS-T100TD (JEOL Ltd.).

TLC analyses were performed on commercial glass plates bearing 0.25-mm layer of Merck Silica gel 60F 254 . Silica gel (Kanto Chemical Co., Silica gel 60 N, spherical, neutral) was used for column chromatography.

IR spectra were measured with a Thermo Scientific iD7 ATR Accessory for the Thermo Scientific Nicolet iS5 FT-IR Spectrometer.

Melting points were measured on an OptiMelt MPA100 automated melting point apparatus (Stanford Research Systems).

CV measurements were recorded with a $\mathrm{CH}$ Instruments: BAS Model 600E Series Electrochemical Analyzer.

UV-Vis absorption spectra were recorded on a Shimadzu UV-1900.

Fluorescence spectra were recorded on a Shimadzu RF-6000.

\section{Reaction set-up and materials}

Kessil PR160L $440 \mathrm{~nm}$ (highest blue and intensity setting) was used as a light source. TEKNOS MG9 was used as a fan.

All reactions were carried out under nitrogen atmosphere. Materials were obtained from commercial suppliers listed as below and stored under nitrogen, and used as received or prepared according to standard procedures unless otherwise noted. 8,9-Dioxa-8a-borabenzo[fg]tetracene (boracene) was prepared by the reported procedure ${ }^{1,2}$ Triazolium salts (N1, N5-N11) were prepared according to the literature. ${ }^{3}$ Imidazolium salt $\mathbf{N 3}$ was prepared according to tha literature. ${ }^{4}$ Thiazolium salt N4 was prepared according to tha literature. ${ }^{5}$ Acyl donors $\left(\mathbf{A} \mathbf{1}^{6}\right.$ and $\left.\mathbf{A 3 ^ { 7 }}\right)$ were prepared according to the literatures. Alkenes $(\mathbf{5 e}, \mathbf{5} \mathbf{f}$ and $\mathbf{5 g})$ were prepared according to the literature. ${ }^{8}$ 


\section{Fujifilm Wako Pure Chemical Co.}

Benzoic Acid, 1,1'-carbonyldiimidazole, cesium carbonate, tetrahydrofuran (Super Dehydrated), 1,4dioxane (Super Dehydrated), Boron Tribromide, o-dichlorobenzene, acetic acid, 2-propanol, dichloromethane (Super Dehydrated), acetone (Super Dehydrated), distilled water, diethylether (Super Dehydrated), 3,5- bis(trifluoromethyl)bromobenzene, bromobenzene, 3-bromobenzoic acid, 4-(4,4,5,5-tetramethyl-1,3,2-dioxaboran-2-yl)benzoic acid, methanol (Super Dehydrated), potassium carbonate, hexane, ethylacetate, ethanol (Super Dehydrated), toluene (Super Dehydrated), benzene (Super Dehydrated), cyclooctane (Super Dehydrated), [1,3-bis(diphenylphosphino) propane]nickel(II) dichloride, bis(pinacolato)diboron, manganese(IV) oxide, tetrahydrofuran-borane (tetrahydrofuran solution), sodium tert-butoxide, acrylonitrile, copper(I) iodide, 4-benzoylbutyric acid, $p$-methoxystyrene, 2-naphthaldehyde, formic acid, paraformaldehyde, sodium perchlorate, sodium hydroxide, N-bromosuccinimide, and N,N'-dicyclohexylcarbodiimide.

\section{Tokyo Chemical Industry Co., Ltd.}

Styrene, $p$-toluic acid, 3,5-dimethylbenzoic acid, 2-naphthoic Acid, 1,3-dibromobenzene, 2,2,6,6tetramethylpiperidine, 1,4dimethyl-1,2,4-triazolium Iodide, 2-bromomesitylene, 2-pyrrolidone, trimethyloxonium tetrafluoroborate, $p$-anisic acid, biphenyl-4-carboxylic acid, sodium tetrafluoroborate, hydrogen chloride (ca. $4 \mathrm{~mol} / \mathrm{L}$ in 1,4-dioxane), 4-chlorobenzoic acid, tetrabutylammonium hexafluorophosphate, bromocyclohexane, triethyl orthoformate, 1,10phenanthroline, 2,6-diethylaniline, 2,6-diisopropylaniline, hydrobromic acid (47\% in water), 5bromo- $m$-xylene, pentafluorophenylhydrazine, indole-2-carboxylic acid, 3-phenylpropionic acid, lithium carbonate, 1-bromo-2-methoxybenzene, pyridine hydrochloride, potassium hydrogenfluoride, 2,3-dimethyl-2-butene, 1-bromoadamantane, ethyl acrylate, ethyl crotonate, ethyl 3-methylcrotonate, 1,4-dibromobutane, potassium 1,3-dioxoisoindolin-2-ide, N,N-dimethylacrylamide, benzyl bromide, estrone, 1,3-dibromopropane, triphenylphosphine, 2,6-dibromopyridine, loxoprofen, cyclohexanecarboxylic acid, pyridine-2-carboxylic acid, 3-thiophenecarboxylic acid, $m$-anisic acid, 4-(trans-4-ethylcyclohexyl)benzoic acid, gemfibrozil, 1-methyl-1-cyclohexanecarboxylic acid, 4chlorostyrene (stabilized with TBC), 4-bromostyrene, methyltriphenylphosphonium bromide, 4(trifluoromethyl)benzaldehyde, methyl terephthalaldehydeate, 2,4,6-trimethylaniline, glyoxal, chlorotrimethylsilane, cycloheptanone, $p$-toluenesulfonic acid, hydrogen peroxide, and (diethylamino)sulfur trifluoride.

\section{Kanto Chemical Co.}

Methyllithium (in cyclopentyl methyl ether $1.09 \mathrm{~mol} / \mathrm{L}$ ), sec-butyllithium (in cyclohexane, $n$-hexane, $1.2 \mathrm{~mol} / \mathrm{L}$ ), $n$-butyllitium (in $1.59 \mathrm{M}$ hexane), tert-butyllithium (in $n$-pentane, $1.6 \mathrm{~mol} / \mathrm{L}$ ), N,Ndimethylformamide (Deoxidized), acetonitrile (Dehydrated), copper(I) chloride, and pentafluorophenol.

\section{Merk Sigma-Aldrich Japan Inc.}

[1,3-Bis(2,6-diisopropylphenyl)imidazol-2-ylidene](3-chloropyridyl)palladium(ii)dichloride, methyl $-d_{3}$-magnesium iodide solution (1.0 M in diethyl ether, 99 atom \% D), benzotrifluoride (Super 
Dehydrated), [(2-biphenyl)dicyclohexylphosphine], di- $\mu$-methoxobis(1,5-cyclooctadiene)diiridium (I), lithium methoxide, and methyl acrylate.

Nacalai Tesque Inc.

1,1'-Carbonyldi(1,2,4-triazole), di-tert-butyl azodicarboxylate, and 2,4,6-trichlorophenylhydrazine.

Chem-impex International.

2-Bromoanisole.

\section{BLD Pharmatech Ltd.}

1,1'-Carbonyldi(1,2,4-triazole), di-tert-butyl azodicarboxylate, 2,4,6-trichlorophenylhydrazine, 1-NBoc-pyrrolidin-2-ylboronic acid, tetrahydroxydiboron, tetrahydropyran-4-carboxylic acid, 3-furoic acid, chromone, and 2-vinylthiophene. 


\section{General Procedure for Synthesis of Boracene-Alkylborates}

\section{Synthesis of tert-Butylborate 1 a as a representative ${ }^{9}$}
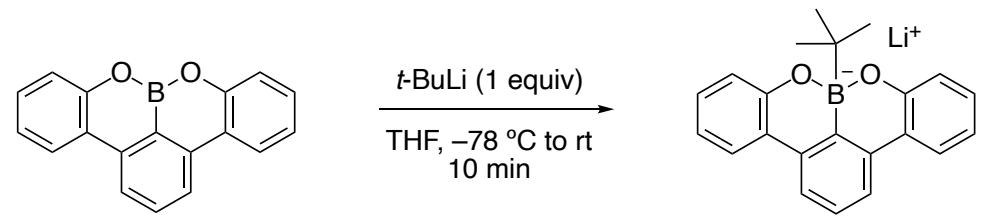

To a suspension of boracene $(270 \mathrm{mg}, 1.00 \mathrm{mmol})$ in THF $(10 \mathrm{~mL})$ was added $t$-butyllithium $(1.6$ $\mathrm{M}$ in $n$-pentane, $0.6 \mathrm{~mL}, 1.0 \mathrm{mmol}$ ) at $-78^{\circ} \mathrm{C}$. After warming to room temperature, the mixture was stirred for $10 \mathrm{~min}$ at the same temperature. The mixture was concentrated under reduced pressure to give $\mathbf{2 a}(475 \mathrm{mg}, 993 \mu \mathrm{mol}, 99.3 \%)$ as a colorless solid.

The molecular weight of 2 a was calculated as [t-Bu(boracene) $] \mathrm{Li} \cdot 2 \mathrm{THF}$ (MW: 478.36) determined from ${ }^{1} \mathrm{H}$ NMR and the X-ray structure, for the use of the experiments. 


\section{General Procedure for Synthesis of PDP-Alkylborates}

Method A (synthesis of 2 a as a representative) ${ }^{10}$
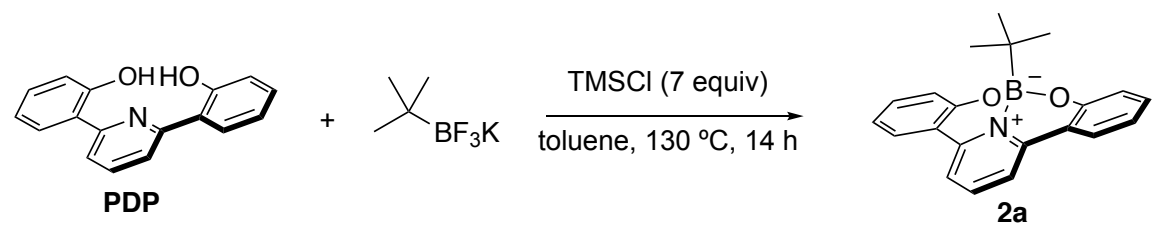

Under an ambient atmosphere, to an oven-dried screw-top $20 \mathrm{~mL}$ vial with a stirring bar was added 2,2'-(pyridine-2,6-diyl)diphenol (263.3 mg, $1.0 \mathrm{mmol}$ ), $t$-BuBF $3 \mathrm{~K}$ (164.0 mg, $1.0 \mathrm{mmol}$ ). The vial was brought to a globebox, and added toluene $(10 \mathrm{~mL})$ and $\mathrm{TMSCl}(884 \mu \mathrm{L}, 7.0 \mathrm{mmol})$. After the vial was removed from the glovebox and sealed with Teflon tape, the vial was placed in aluminum heating block at $130{ }^{\circ} \mathrm{C}$ and stirred for $14 \mathrm{~h}$. After cooling to room temperature, the reaction crude was passed through a short pad of $\mathrm{N}-\mathrm{H}$ silica gel with toluene followed by evaporation and vacuum pump to remove the solvent to give PDP-borate 2a as a yellow solid (315.9 mg, $0.960 \mathrm{mmol}, 96 \%$ yield).

Method B (synthesis of $2 \mathrm{c}$ as a representative) ${ }^{10}$
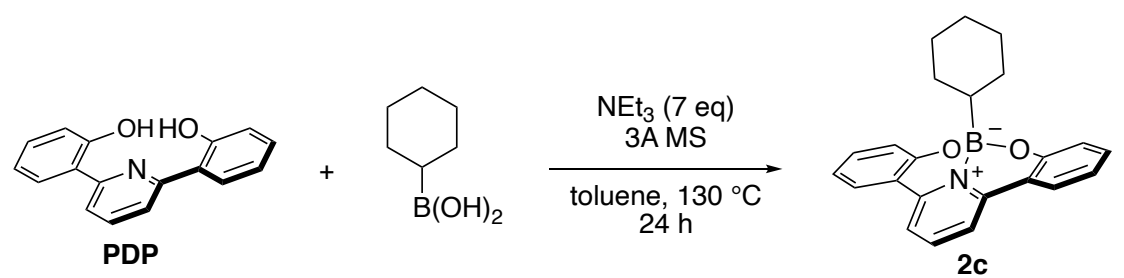

Under an ambient atmosphere, to an oven-dried screw-top $20 \mathrm{~mL}$ vial with a stirring bar was added 2,2'-(pyridine-2,6-diyl)diphenol (263.3 mg, $1.0 \mathrm{mmol}$ ), cyclohexylboronic acid (114.0 mg, $1.0 \mathrm{mmol}$ ). The vial was brought to a globebox, and added molecular sieve $3 \mathrm{~A}(100 \mathrm{mg})$, toluene $(3.3 \mathrm{~mL})$ and triethylamine $(976 \mu \mathrm{L}, 7.0 \mathrm{mmol})$. After the vial was removed from the glovebox and sealed with Teflon tape, the vial was placed in aluminum heating block at $130{ }^{\circ} \mathrm{C}$ and stirred for $24 \mathrm{~h}$. After cooling to room temperature, the reaction crude was passed through a short pad of Celite followed by evaporation and vacuum pump remove the solvent to give PDP-borate $\mathbf{2 c}$ as a yellow solid (344 mg, $0.968 \mathrm{mmol}, 97 \%)$. 


\section{Detailed Optimization Conditions for Two-Component Coupling}

Table S1. Screening of NHC catalysts

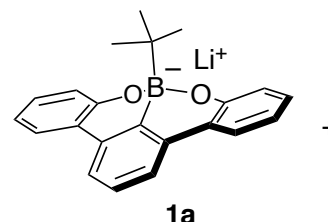

$1 a$<smiles>Cc1ccc(C(=O)n2ccnc2)cc1</smiles>

3a

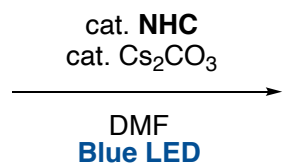

Blue LED<smiles>Cc1ccc(C(=O)C(C)(C)C)cc1</smiles>

4aa

\begin{tabular}{ccc} 
Entry & NHC catalyst & ${\text { Yield of 4aa }(\%)^{a}}^{a}$ \\
\hline $\mathbf{1}$ & $\mathbf{N 1}$ & $\mathbf{5 8}$ \\
2 & $\mathbf{N 2}$ & 14 \\
3 & $\mathbf{N 3}$ & 17 \\
4 & $\mathbf{N 4}$ & 15 \\
5 & $\mathbf{N 5}$ & 40 \\
6 & $\mathbf{N 6}$ & 38 \\
7 & $\mathbf{N 7}$ & 17 \\
8 & $\mathbf{N 8}$ & 29 \\
9 & $\mathbf{N 9}$ & 19 \\
10 & $\mathbf{N 1 0}$ & 8 \\
11 & $\mathbf{N 1 1}$ & 7 \\
$12^{b}$ & $\mathbf{N 1}$ & 46 \\
$13^{c}$ & $\mathbf{N 1}$ & 31 \\
14 & w/o NHC & 16
\end{tabular}

${ }^{a}$ Reaction was carried out with $1 \mathbf{a}(0.12 \mathrm{mmol}), \mathbf{3 a}(0.1 \mathrm{mmol})$, NHC catalyst $(0.015 \mathrm{mmol})$ and $\mathrm{Cs}_{2} \mathrm{CO}_{3}(0.015 \mathrm{mmol})$ in DMF (1 mL) under $440 \mathrm{~nm}$ blue LED (Kessil lamp) irradiation at ambient temperature for 6 h. ${ }^{b} \mathbf{N} \mathbf{1}(0.010 \mathrm{mmol})$ and $\mathrm{Cs}_{2} \mathrm{CO}_{3}(0.010 \mathrm{mmol})$ were used. ${ }^{c} \mathbf{N} 1(0.030 \mathrm{mmol})$ and $\mathrm{Cs}_{2} \mathrm{CO}_{3}(0.030 \mathrm{mmol})$ were used.

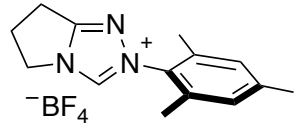

N1

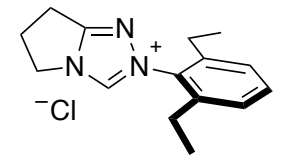

N5

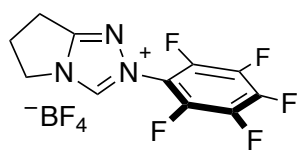

N9<smiles>Cn1cn[n+](C)c1</smiles>

N2

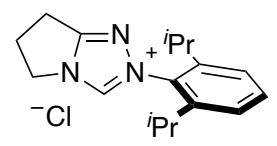

N6

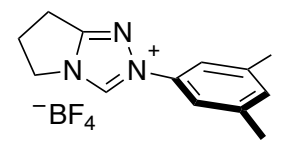

N10

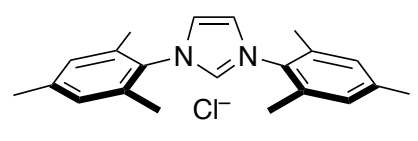

N3

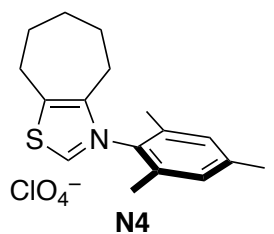

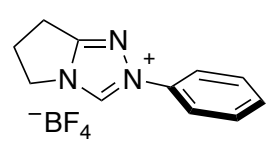

N7

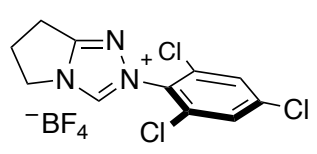

N8

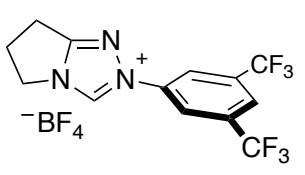

N11 
Table S2. Effect of base

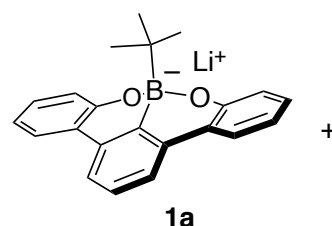<smiles>Cc1ccc(C(=O)n2ccnc2)cc1</smiles>

3a

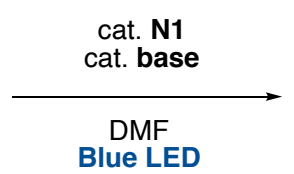<smiles>Cc1ccc(C(=O)C(C)(C)C)cc1</smiles>

4aa

\begin{tabular}{ccc} 
Entry & Base & Yield of 4aa $(\%)^{a}$ \\
\hline $\mathbf{1}$ & $\mathrm{Cs}_{2} \mathrm{CO}_{3}$ & $\mathbf{5 8}$ \\
2 & $\mathrm{Li}_{2} \mathrm{CO}_{3}$ & 29 \\
3 & $\mathrm{Na}_{2} \mathrm{CO}_{3}$ & 26 \\
4 & $\mathrm{~K}_{2} \mathrm{CO}_{3}$ & 26 \\
5 & $\mathrm{CsOAc}$ & 28 \\
\hline
\end{tabular}

${ }^{a}$ Reaction was carried out with $1 \mathbf{a}(0.12 \mathrm{mmol}), \mathbf{3 a}(0.1 \mathrm{mmol}), \mathbf{N 1}(0.015 \mathrm{mmol})$ and base $(0.015 \mathrm{mmol})$ in DMF $(1 \mathrm{~mL})$ under $440 \mathrm{~nm}$ blue LED (Kessil Lamp) irradiation at ambient temperature for $6 \mathrm{~h}$.

Table S3. Effect of solvent

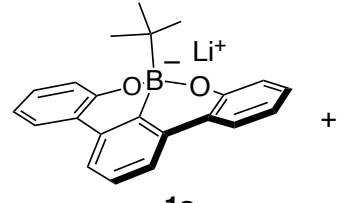

$1 \mathrm{a}$<smiles>Cc1ccc(C(=O)n2ccnc2)cc1</smiles>

$3 a$

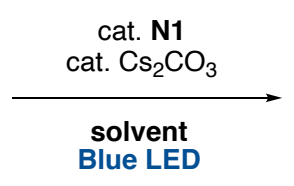

Blue LED<smiles>Cc1ccc(C(=O)C(C)(C)C)cc1</smiles>

4aa

\begin{tabular}{ccc} 
Entry & Solvent & ${\text { Yield of 4aa }(\%)^{a}}^{a}$ \\
\hline $\mathbf{1}$ & DMF & $\mathbf{5 8}$ \\
2 & MeCN & 44 \\
3 & $\mathrm{THF}$ & 33 \\
4 & $\mathrm{DCM}$ & 13 \\
5 & $\mathrm{DMA}$ & 52 \\
6 & $\mathrm{DMSO}$ & 44 \\
7 & $\mathrm{MeOH}$ & 0 \\
8 & $\mathrm{C}_{6} \mathrm{H}_{5} \mathrm{CF}$ & 9 \\
9 & $\mathrm{DMF} / \mathrm{MeOH}(1 / 1)$ & 0 \\
10 & $\mathrm{DMF} / \mathrm{DCM}(1 / 1)$ & 32 \\
$11^{b}$ & $\mathrm{DMF}$ & 50 \\
$12^{c}$ & $\mathrm{DMF}$ & 49 \\
\hline
\end{tabular}

${ }^{a}$ Reaction was carried out with $1 \mathbf{a}(0.12 \mathrm{mmol}), \mathbf{3 a}(0.1 \mathrm{mmol}), \mathbf{N 1}(0.015 \mathrm{mmol})$ and $\mathrm{Cs}_{2} \mathrm{CO}_{3}(0.015 \mathrm{mmol})$ in solvent $(1$ $\mathrm{mL}$ ) under $440 \mathrm{~nm}$ blue LED (Kessil Lamp) irradiation at ambient temperature for $6 \mathrm{~h} .{ }^{b} \mathrm{DMF}(2.0 \mathrm{~mL}) \mathrm{was}$ used. ${ }^{c} \mathrm{DMF}$ $(500 \mu \mathrm{L})$ was used. 


\section{General Procedure for Two-Component Coupling}

(1)<smiles>Cc1ccc(C(=O)O)cc1</smiles>

(2)

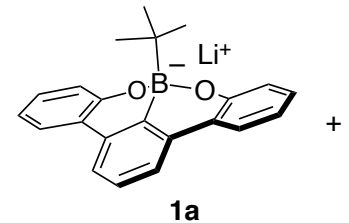<smiles></smiles>

DCM, r.t., $12 \mathrm{~h}$<smiles>Cc1ccc(C(=O)n2ccnc2)cc1</smiles>

$3 a$

Blue LED<smiles>Cc1ccc(C(=O)n2ccnc2)cc1</smiles>

$3 a$

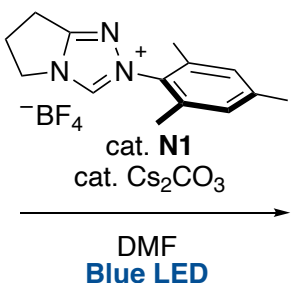

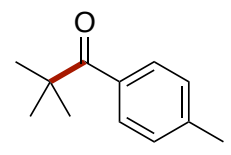

4aa

The reaction to produce $4 a$ a in Table 1 is representative. According to the literature, ${ }^{11}$ acyl imidazole 3a was prepared. To a suspension of $p$-toluic acid $(13.6 \mathrm{mg}, 0.1 \mathrm{mmol})$ in dry dichloromethane $(333 \mu \mathrm{L})$ was added slowly carbonyldiimidazole (CDI, $24.3 \mathrm{mg}, 0.15 \mathrm{mmol}, 1.5$ equiv) (caution, exothermic). After stirring for $12 \mathrm{~h}$ at room temperature, the resulting solution was transferred to separatory funnel and washed with deionized water (ca. $5 \mathrm{~mL}$ ). The organic extract was dried over $\mathrm{Na}_{2} \mathrm{SO}_{4}$ and after filtration, the filtrate was concentrated under reduced pressure to afford the acyl imidazole 3a (18.6 mg, $0.1 \mathrm{mmol}$, quantitative). The acyl imidazole was used without further purification.

Triazolium salt N1 $(4.7 \mathrm{mg}, 15 \mu \mathrm{mol})$, borate $1 \mathbf{a}(57.4 \mathrm{mg}, 0.12 \mathrm{mmol}), \mathrm{Cs}_{2} \mathrm{CO}_{3}(4.9 \mathrm{mg}, 15 \mu \mathrm{mol})$, and acyl imidazole $3 \mathbf{a}(18.6 \mathrm{mg}, 0.1 \mathrm{mmol})$ were placed in an oven-dried screw-top $5 \mathrm{~mL}$ vial containing a magnetic stirring bar. The vial was sealed by a hole cap with rubber septum, and then sparged with nitrogen, followed by addition of degassed DMF $(1 \mathrm{~mL})$. After $6 \mathrm{~h}$ stirring at ambient temperature under photoirradiation $(440 \mathrm{~nm})$, the reaction mixture was quenched with water $(1 \mathrm{~mL})$, then extracted with diethyl ether (4 times) and dried over sodium sulfate. After filtration was evaporated under reduced pressure. After the volatiles were removed under reduced pressure, flash column chromatography on silica gel (100:0-98:2, hexane/Et $2 \mathrm{O})$ gave 4aa (9.2 mg, $0.052 \mathrm{mmol})$ in $52 \%$ yield.
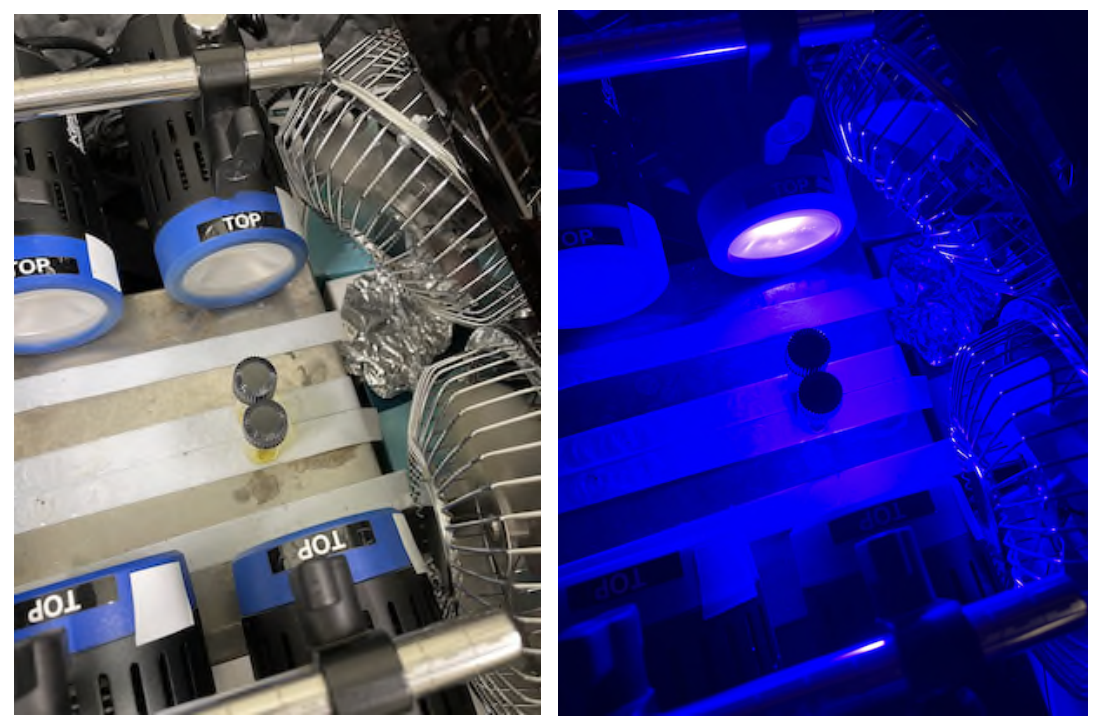

Figure S1. Light set up. 


\section{Cyclic Voltammetry of Acyl Azolium Intermediates}

The preparation of INT-I is representative. In a glovebox, to an oven-dried vial with a stirring bar was added acyl imidazole 3a (1.86 mg, $10 \mu \mathrm{mol})$, N1 (3.15 mg, $10 \mu \mathrm{mol})$ and $\mathrm{Cs}_{2} \mathrm{CO}_{3}(3.26 \mathrm{mg}$, $10 \mu \mathrm{mol})$. To the vial, $5.0 \mathrm{~mL}$ of $\mathrm{MeCN}$ with tetrabutylammonium hexafluorophosphate $\left([\mathrm{N}(n \mathrm{Bu})]_{4} \mathrm{PF}_{6}\right)(193.7 \mathrm{mg}, 0.5 \mathrm{mmol}, 0.1 \mathrm{M})$ dissolved as a supporting electrolyte was added, and the mixture was stirred for $2 \mathrm{~h}$ at room temperature. Then, the ressulting solution was transffered to the measuring vessel, and the electrochemical property of the ground state acyl azolium INT-I was mesured.

Measurements used a glassy carbon working electrode (area $=0.07 \mathrm{~cm}^{2}$ ), a $\mathrm{Ag} / \mathrm{AgCl}$ reference electrode (SCE), and a Pt wire counter electrode. The concentration of the sample solution was fixed at $2 \mathrm{mM}$ and the sweep rates were set to $100 \mathrm{mV} / \mathrm{s}$.
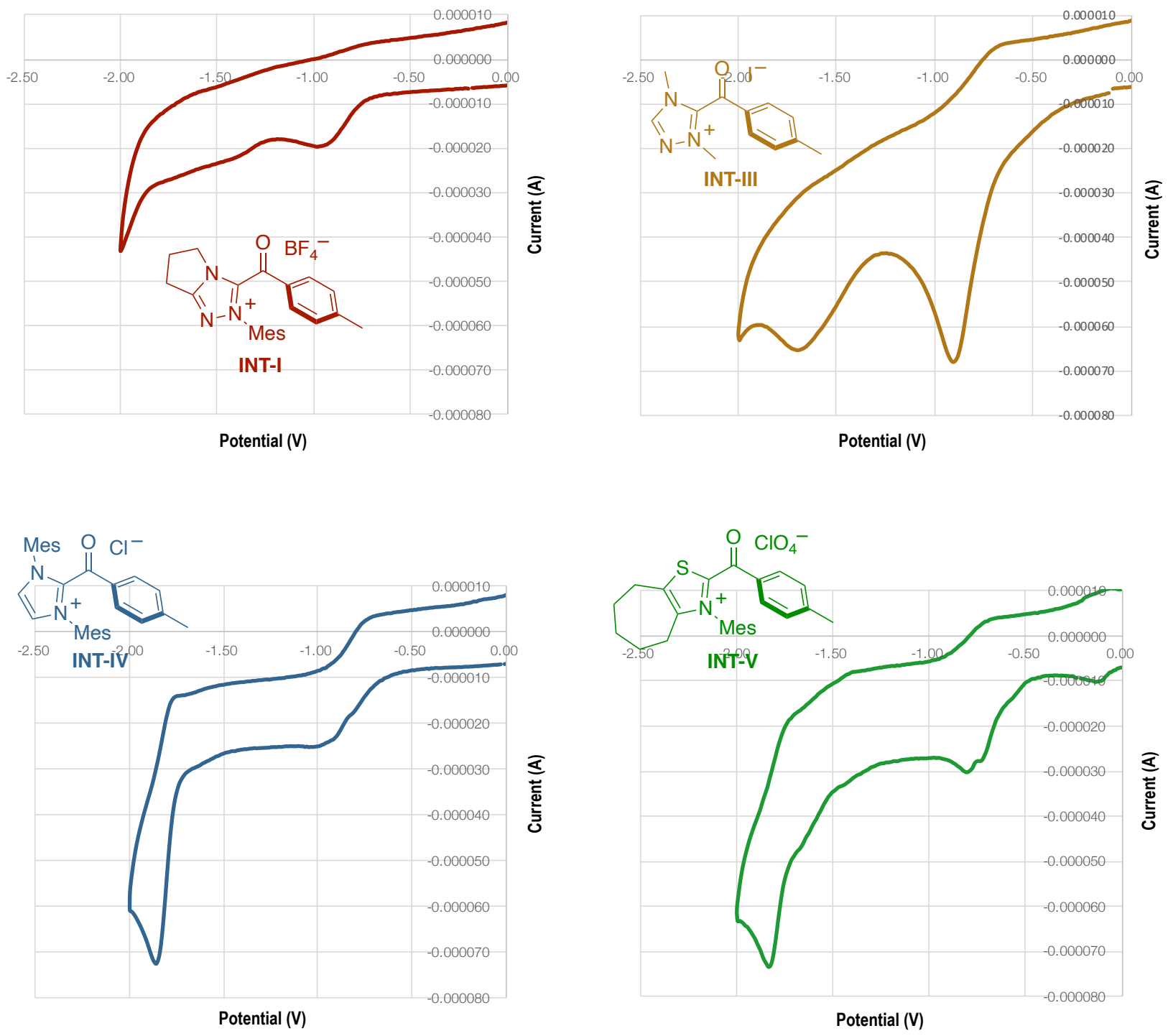

Figure S2. Cyclic voltammogram of acyl azolium intermediates.

The result of cyclic voltammetry suggested that no significant difference in the reduction potentials was observed about the acyl azolium intermediates derived from NHC catalysts. Thus, all intermediates can undergo effiently single-electron reduction from the excited borate $1 \mathbf{a}\left(E_{1 / 2}=\mathrm{ca}\right.$. 2.2 V vs. SCE in $\mathrm{MeCN}$ ). 


\section{UV/Vis specta of Reaction Substrates}

- UV/vis absorption spectra of borate 1a, acyl imidazole 3a and the pre-formed acyl azolium intermediate INT-I were measured with $0.1 \mathrm{mM}$ DMF solution.

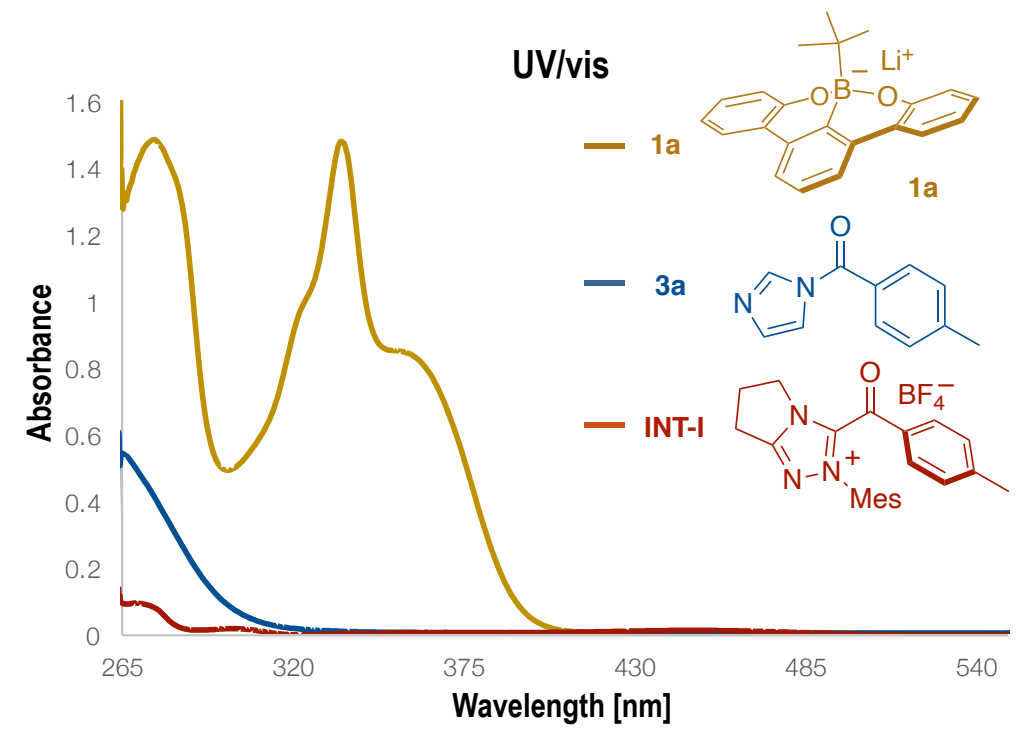

Figure S3. Overlaid UV/vis absorption spectra of 1a, 3a and INT-I.

- UV/vis absorption spectra of mixed solution (borate 1a : acyl azolium INT-I $=1: 1$ or borate 1a : acyl imidazole 3a $=1: 1$ ) were measured with $0.1 \mathrm{mM}$ DMF.
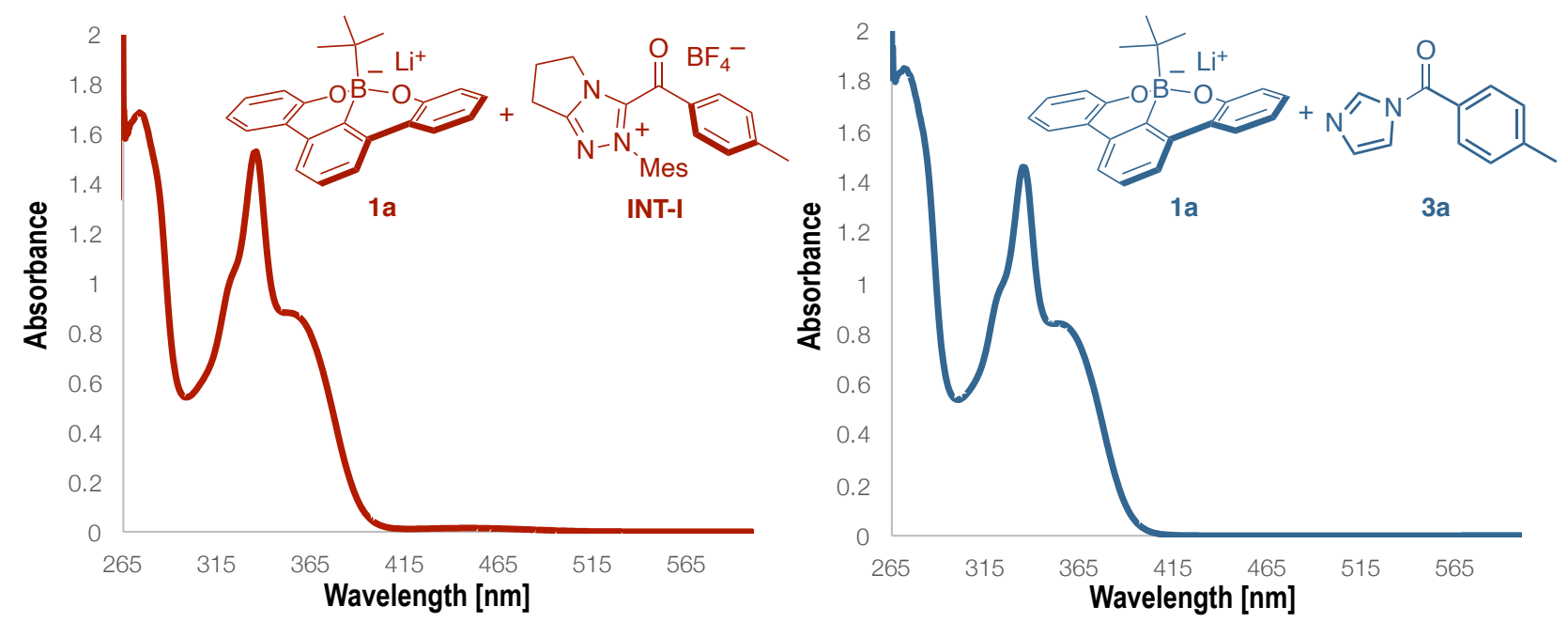

Figure S4. UV/vis absorption spectra of mixed solution (borate 1a : acyl azolium INT-I $=1: 1$ or borate 1a : acyl imidazole 3a $=1: 1$ ). Red line: mixed solution (borate $\mathbf{1 a}$ : acyl azolium $\mathbf{I N T}-\mathbf{I}=1: 1$ ) in $0.1 \mathrm{mM}$ DMF. Blue line: mixed solution (borate 1a : acyl imidazole 3a=1:1) in 0.1 mM DMF.

The result of UV/vis spectra of mixed solution indicated that among the three components (1a, 3a and INT-I), borate 1a could be most efficiently excited by visible light in $0.1 \mathrm{mM} \mathrm{DMF}$. 
-UV/vis absorption spectra of borate 1a and acyl azolium INT-I were measured with $0.1 \mathrm{M}$ or 15 mM DMF solution.

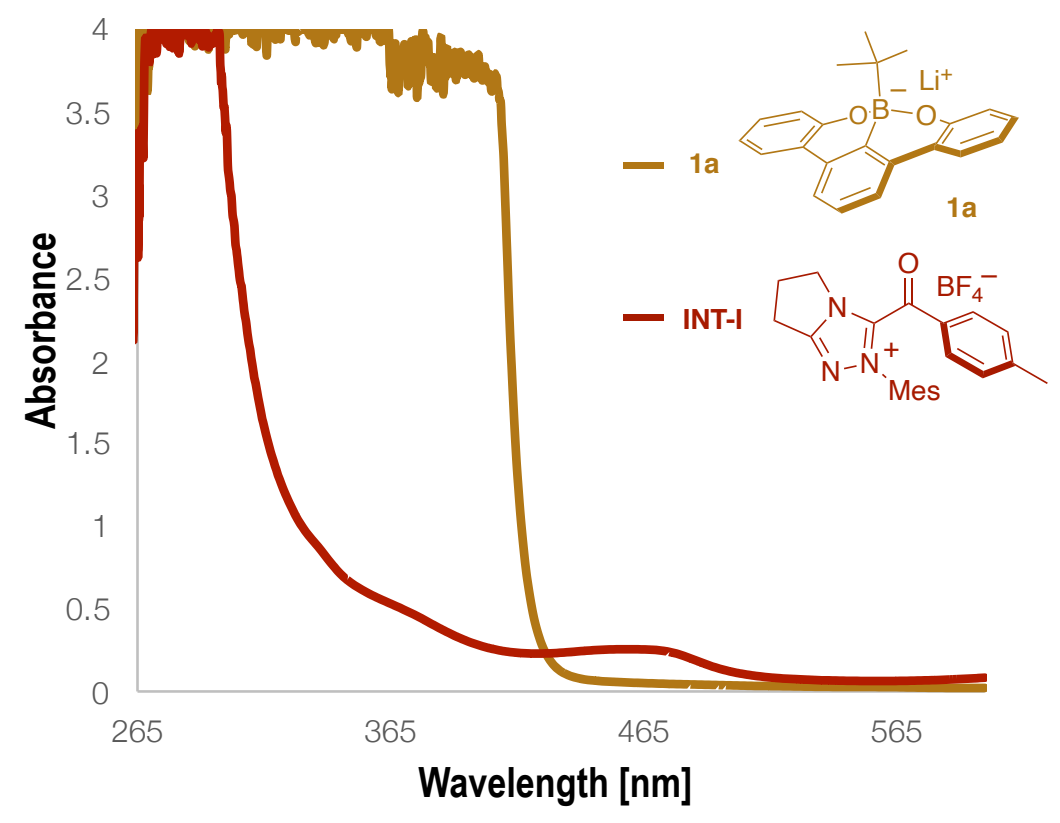

Figure S5. UV/vis spectra of 1a (0.1 M in DMF) and INT-I (15 mM in DMF)

- UV/vis absorption spectra of mixed solution (borate 1a : acyl azolium INT-I = 1: 0.15) was measured with $0.1 \mathrm{mM}$ DMF.

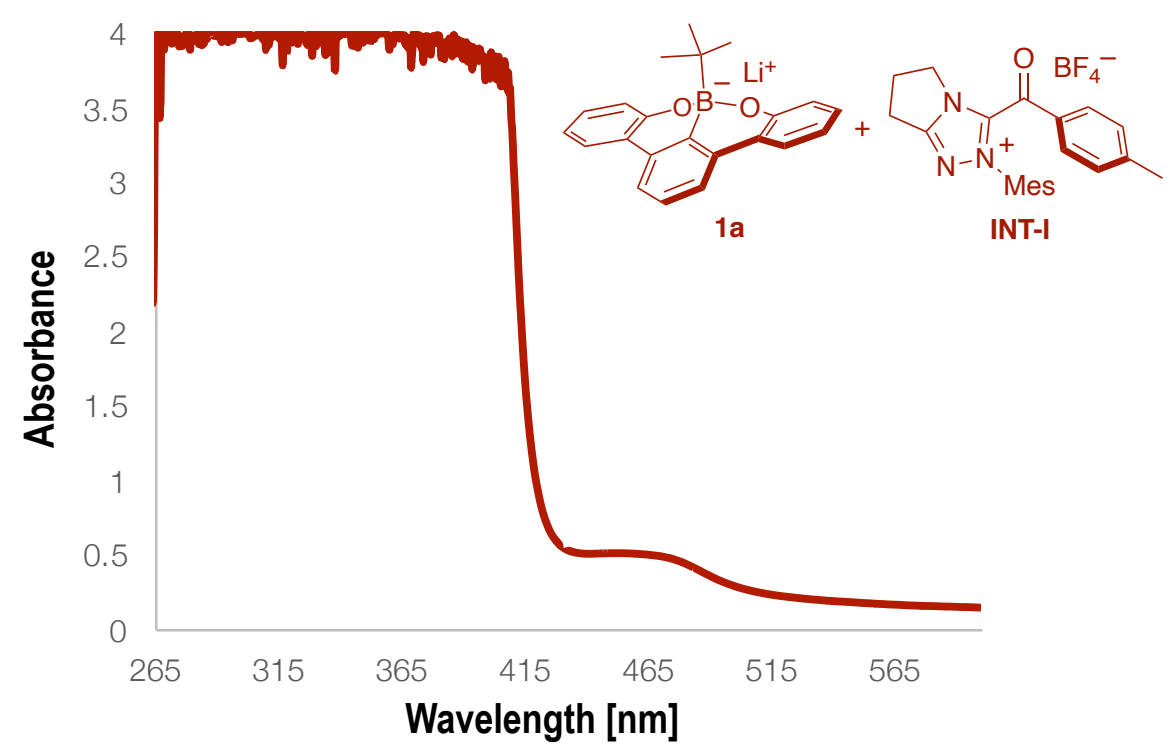

Figure S6. UV/vis absorption spectra of mixed solution (1a : INT-I = 1: 0.15).

The UV/vis spectra of the mixed solution suggested that both the borate 1a and acyl azolium INTI can absorb visible light at high concentrations. While both 1a and INT-I could be excited, their absorption intensities indicated that borate 1 a could be more efficiently excited than INT-I. 


\section{Photo-quenching Experiment: Stern-Volmer Plot}

In a typical experiment, the fluoresence intensity was measured using a screw-top quartz cuvette (10 mm light path). The sample was prepared by $100 \mu \mathrm{L}$ of $2 \mathrm{mM}$ solution of borate $1 \mathrm{a}$ in degassed $\mathrm{MeCN}$ with degassed MeCN solution of acyl azolium INT-I or acyl imidazole 3a (quencher) in each concentrations $(600,1000,1400,1600$, or $1800 \mathrm{mM}$, respectively) and added until the final volume $(2.0 \mathrm{~mL})$. The fluoresence intensity was measured excited absorption maximum $\left(\lambda_{\max }=335 \mathrm{~nm}\right)$ and the intensity value was determined by the corresponding value of emission maximum $\left(E_{\max }=386\right.$ $\mathrm{nm})$.

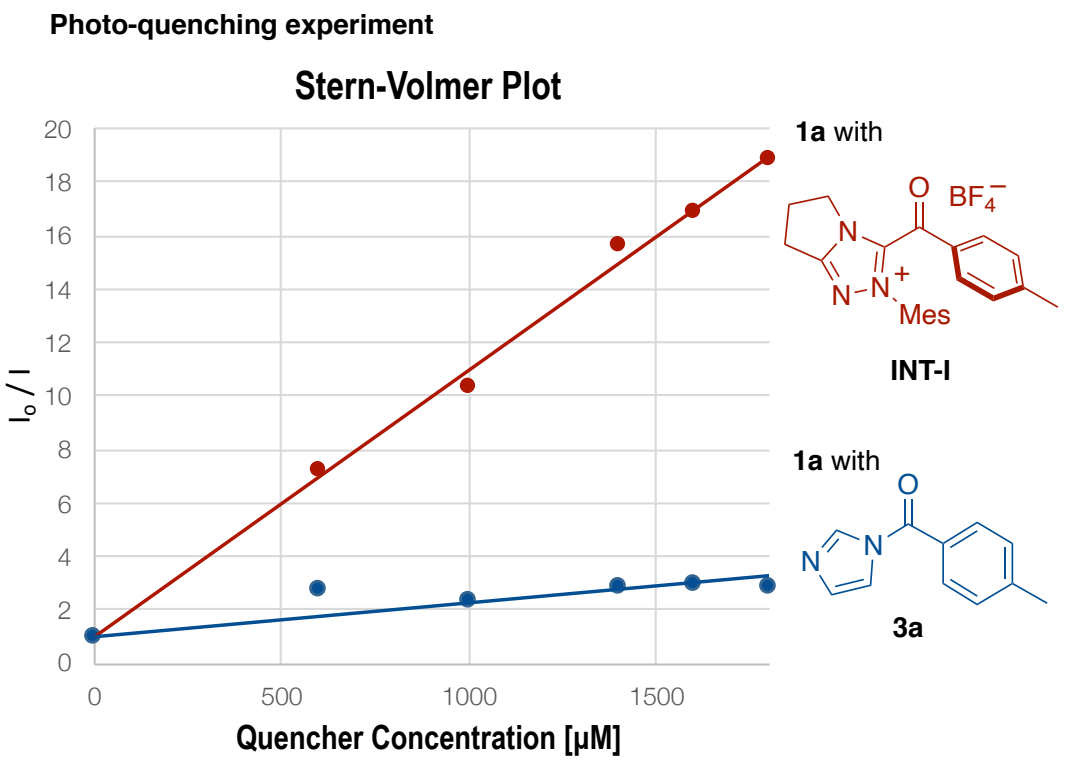

Figure S7. Stern-Volmer Quenching Studies.

The result of Stern-Volmer experiment indicated that the excited borate 1a was quenched more effciently by acyl azolium INT-I than acyl imidazole 3a. Therefore, the single-electron transfer would proceed from the excited borate 1a to acyl azolium INT-I in the reaction. 


\section{Stoichiometric Experiment}

\section{Preparation of INT-I}<smiles>Cc1ccc(C(=O)n2ccnc2)cc1</smiles>

3a

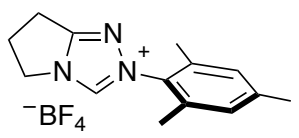

N1

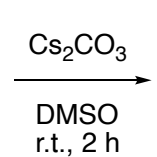

.t., $2 \mathrm{~h}$

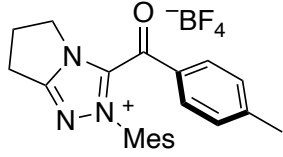

INT-I

In a glovebox, to an oven-dried vial with a stirring bar was added acyl imidazole 3a (18.6 mg, 0.1 mmol), Triazolium salt N1 $(31.5 \mathrm{mg}, 0.1 \mathrm{mmol}), \mathrm{Cs}_{2} \mathrm{CO}_{3}(32.6 \mathrm{mg}, 0.1 \mathrm{mmol})$ and DMSO- $d_{6}(1.0$ $\mathrm{mL}$ ). After stirring for $2 \mathrm{~h}$ at room temperature, the formation of INT-I was confirmed by ${ }^{1} \mathrm{H}$ NMR analysis (Figure S8).

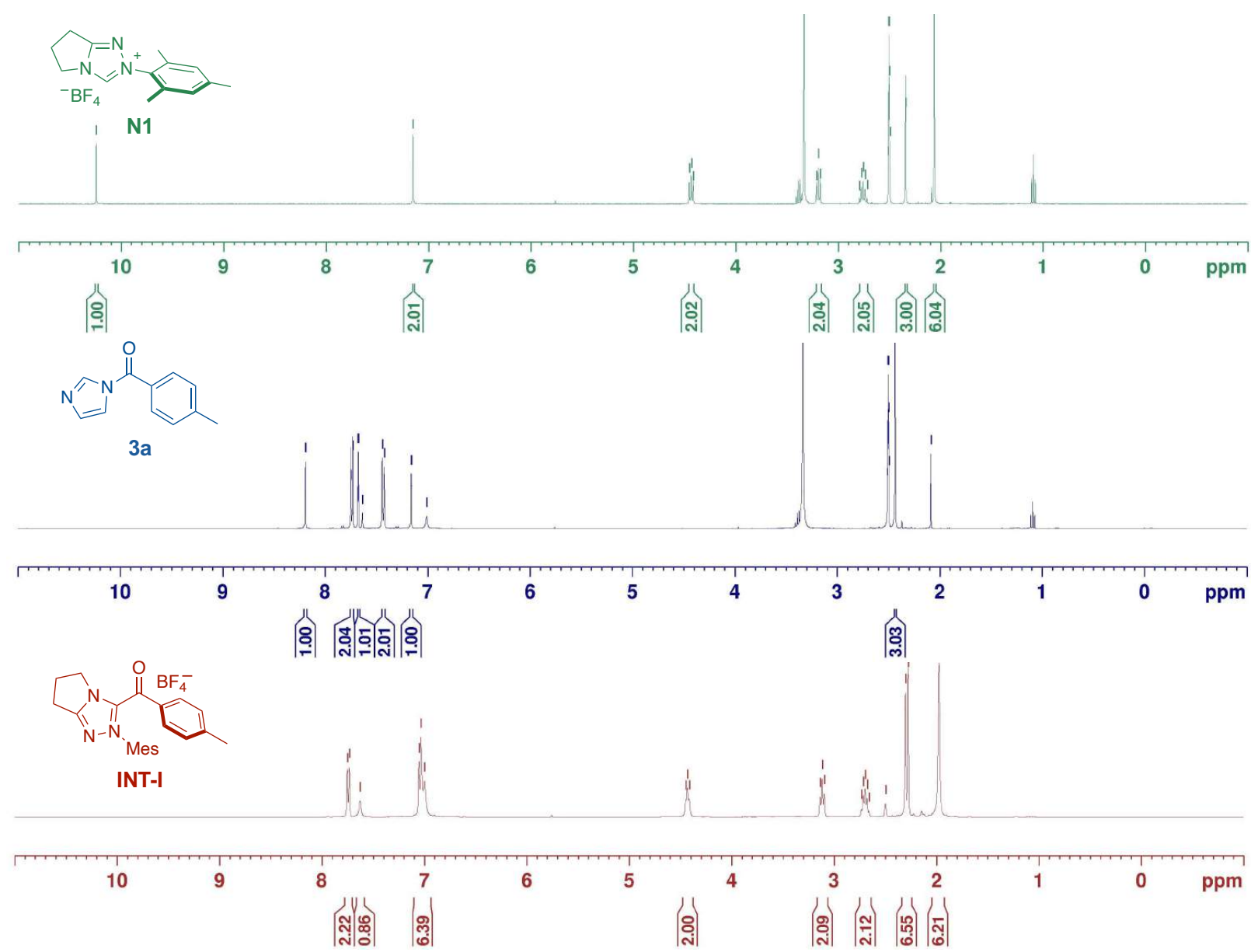

Figure S8. Overlaid ${ }^{1} \mathrm{H}$ NMR spectra $\left(\mathrm{DMSO}-d_{6}\right)$ of N1, 3a, and INT-I. 
Stoichiometric reaction of INT-I with 1a

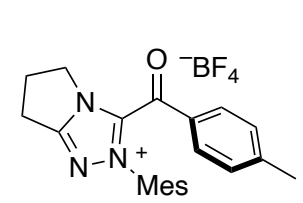

INT-I

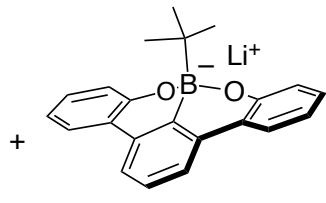

1a<smiles>Cc1ccc(C(=O)C(C)(C)C)cc1</smiles>

4aa not observed<smiles>BrC12CC=CC3=C1B(Oc1ccccc13)Oc1ccccc12</smiles>

by-product 7

After preparation of INT-I, to the solution was added borate 1a $(57.4 \mathrm{mg}, 0.12 \mathrm{mmol})$. After $6 \mathrm{~h}$ stirring at ambient temperature under photoirradiation $(440 \mathrm{~nm})$, the reaction mixture was quenched with water $(1 \mathrm{~mL})$, then extracted with diethyl ether (4 times) and dried over sodium sulfate.

While the product 4aa was not observed in ${ }^{1} \mathrm{H}$ NMR analysis, the by-product 7 was formed in $11 \%$ ${ }^{1} \mathrm{H}$ NMR yield. The by-product 7 combined from several stoichiometric experiments was identified by $1 \mathrm{D}-\mathrm{NMR}\left({ }^{1} \mathrm{H},{ }^{13} \mathrm{C}\right.$, DEPT135 and $\left.{ }^{11} \mathrm{~B}\right)$ and 2D-NMR (H-H COSY, HMQC and HMBC).

This result could be attributed to that the borate 1a was not efficiently excited. As mentioned earlier, the high concentration of acyl azolium intermediate INT-I absorbs visible light. Thus, the stoichiometric amounts of INT-I could affect the direct excitation of borate 1a, which caused no desired radical-radical coupling.
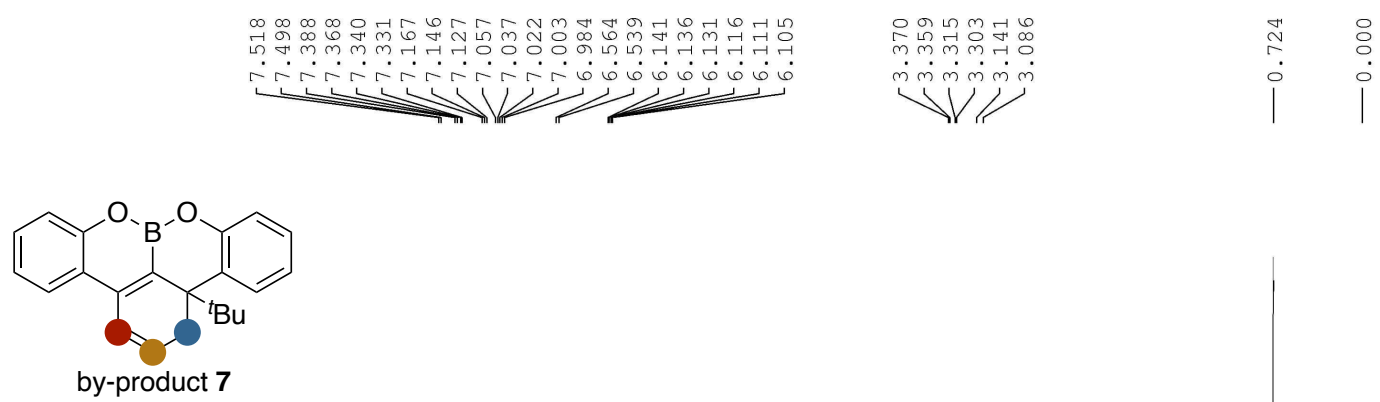

by-product 7

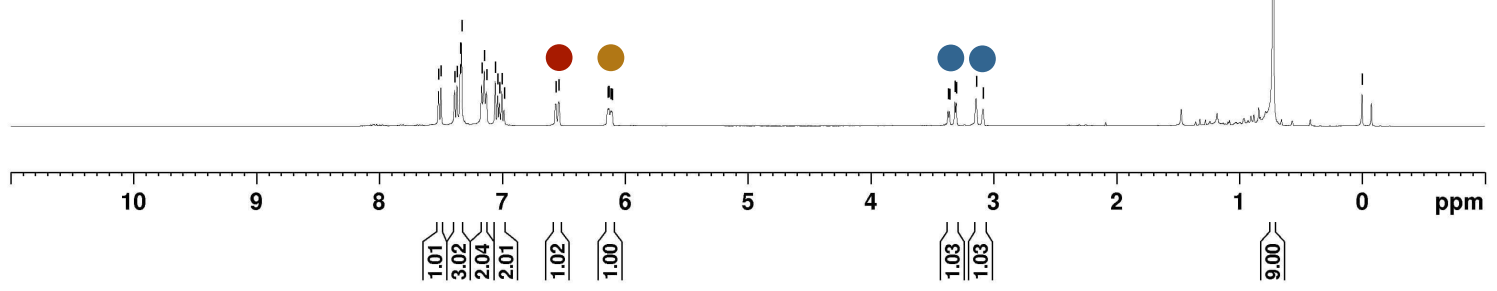

Figure S9. ${ }^{1} \mathrm{H} \mathrm{NMR}\left(\mathrm{CDCl}_{3}\right)$ of by-product 7 . 

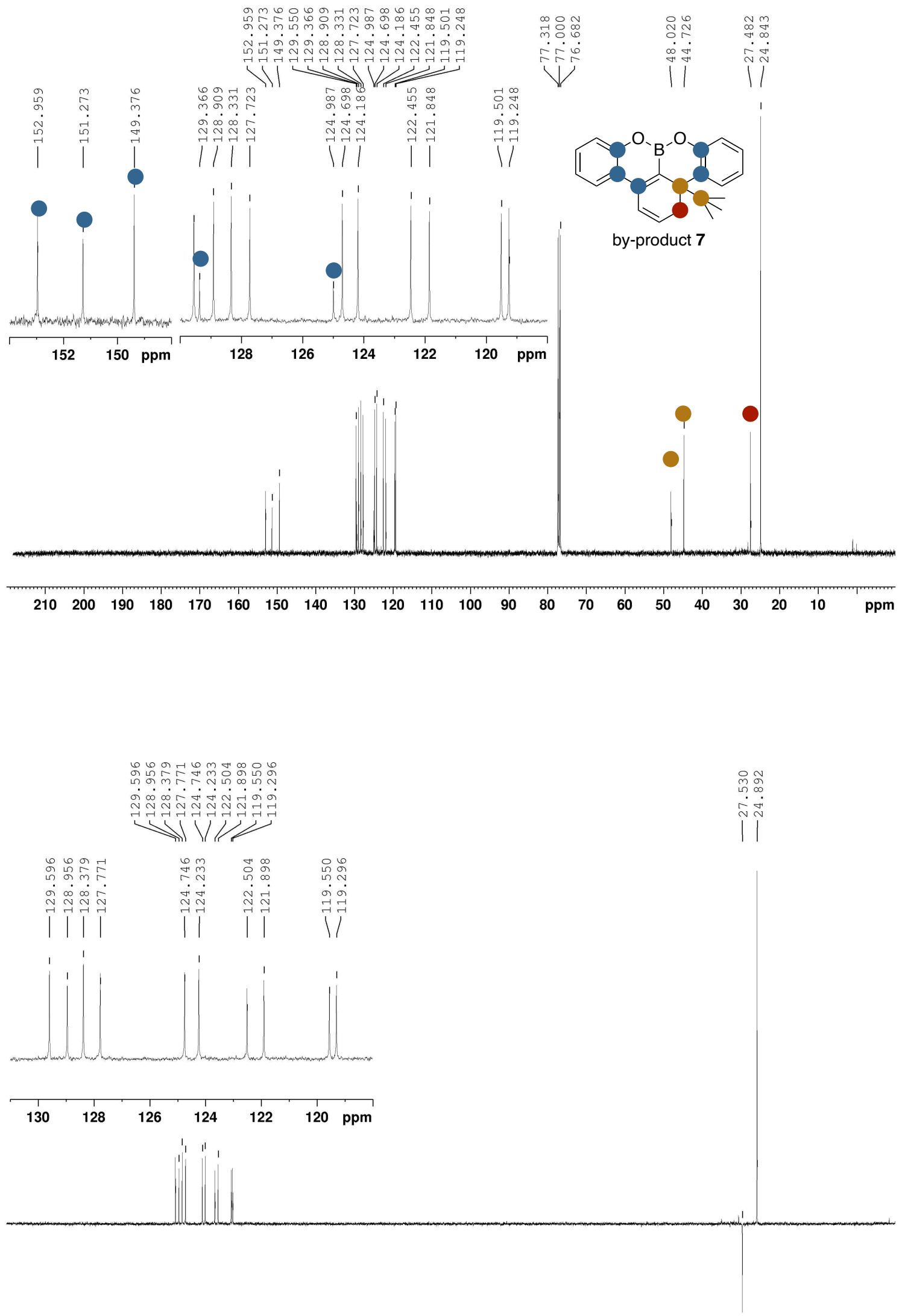

$\begin{array}{llllllllllllllll}150 & 140 & 130 & 120 & 110 & 100 & 90 & 80 & 70 & 60 & 50 & 40 & 30 & 20 & 10 & \mathrm{ppm}\end{array}$

Figure S10. ${ }^{13} \mathrm{C}$ NMR and DEPT135 $\left(\mathrm{CDCl}_{3}\right)$ of by-product 7. 


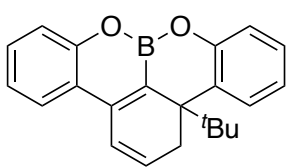

by-product 7

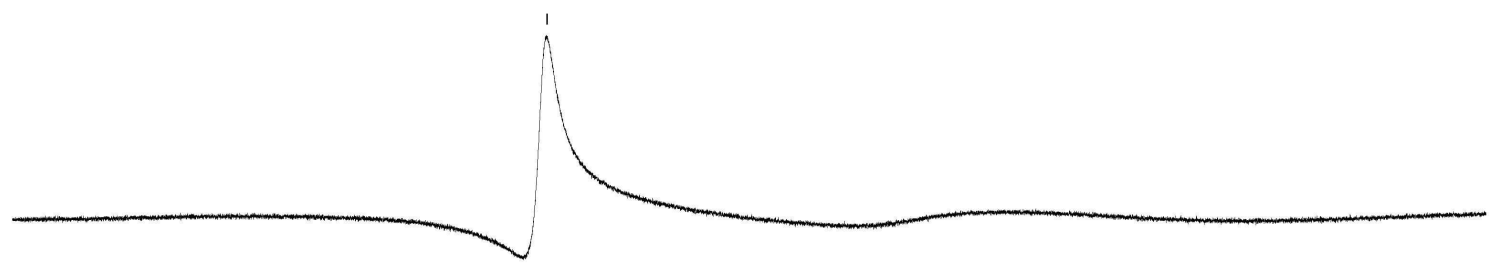

$\begin{array}{lllllllllllllllllll}90 & 80 & 70 & 60 & 50 & 40 & 30 & 20 & 10 & 0 & -10 & -20 & -30 & -40 & -50 & -60 & -70 & -80 & \mathrm{ppm}\end{array}$

Figure S11. ${ }^{11} \mathrm{~B} \mathrm{NMR}\left(\mathrm{CDCl}_{3}\right)$ of by-product 7. 


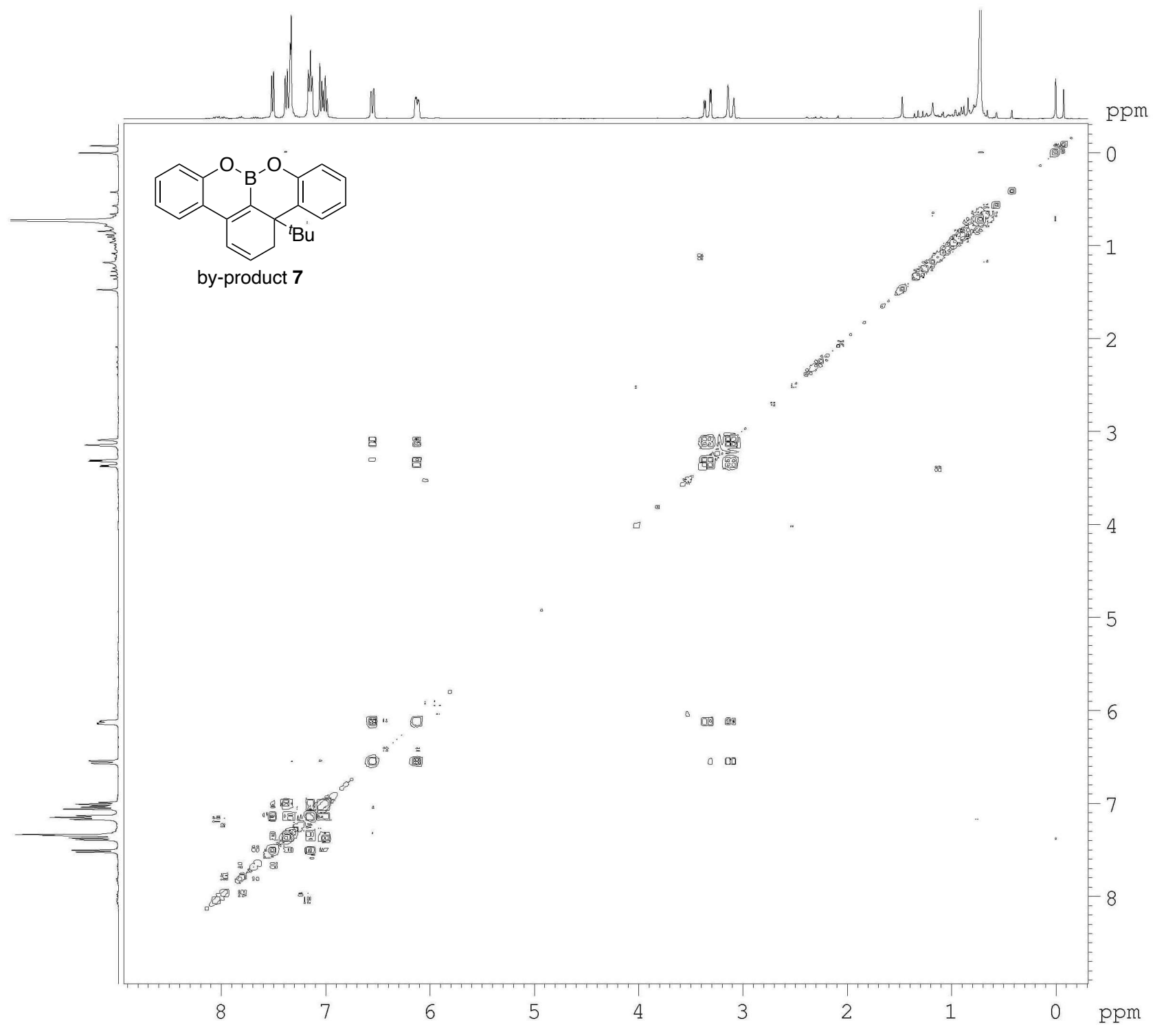

Figure S12. H-H COSY $\left(\mathrm{CDCl}_{3}\right)$ of by-product 7. 


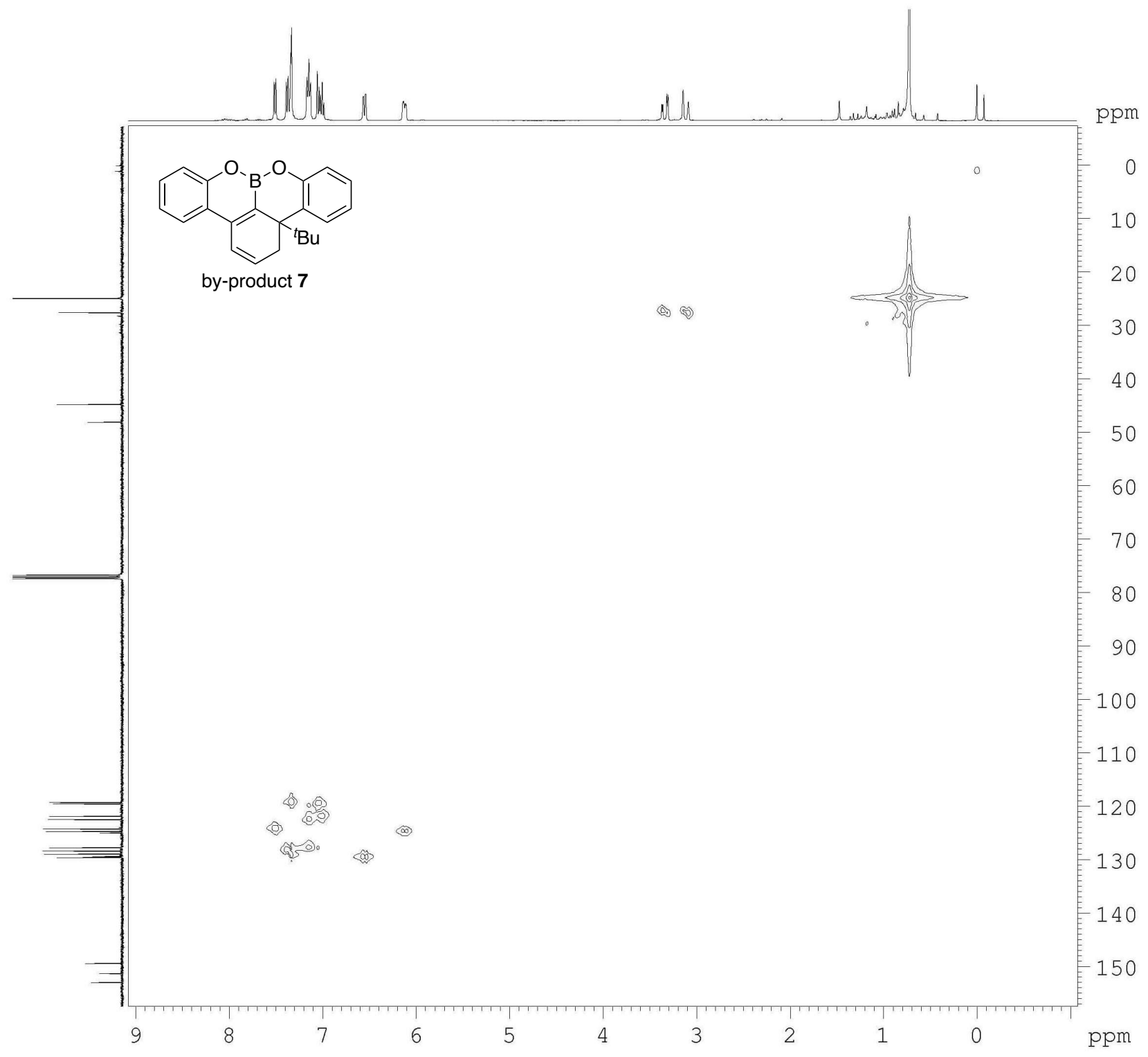

Figure S13. HMQC $\left(\mathrm{CDCl}_{3}\right)$ of by-product 7. 


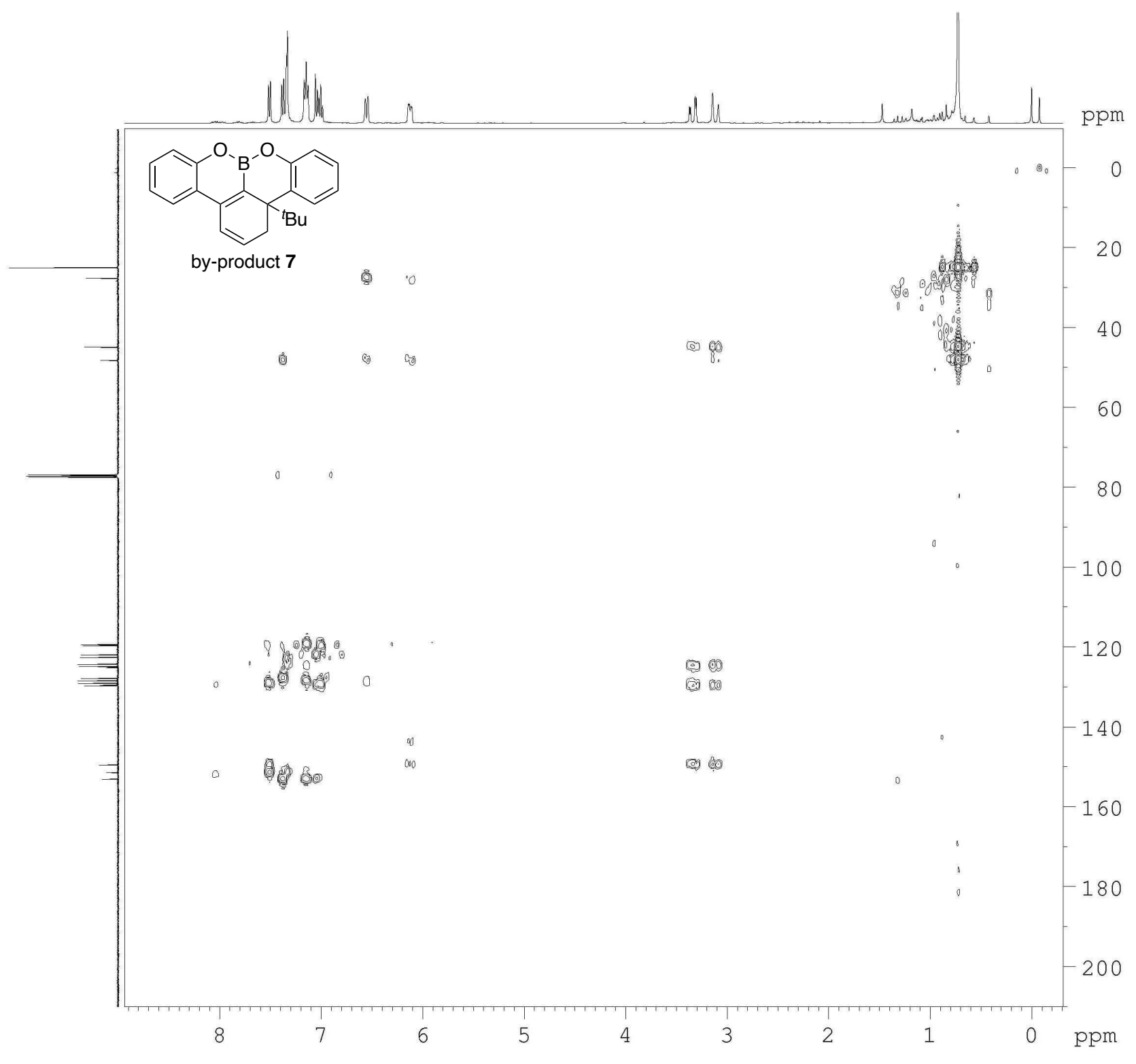

Figure S14. HMBC $\left(\mathrm{CDCl}_{3}\right)$ of by-product 7 . 


\section{2,2-Dimethyl-1-(p-tolyl)propan-1-one (4aa)}<smiles>Cc1ccc(C(=O)C(C)(C)C)cc1</smiles>

The product 4aa was purified by flash chromatography on silica gel (100:0-98:2, hexane/Et $\left.{ }_{2} \mathrm{O}\right)$ (Table 1; $9.2 \mathrm{mg}, 0.052 \mathrm{mmol}, 52 \%$ isolated yield).

${ }^{1}$ H NMR $\left(400 \mathrm{MHz} \mathrm{CDCl}_{3}\right) \delta 7.66(\mathrm{~d}, J=7.6 \mathrm{~Hz}, 2 \mathrm{H}), 7.20(\mathrm{~d}, J=7.6 \mathrm{~Hz}, 2 \mathrm{H}), 2.38(\mathrm{~s}, 3 \mathrm{H}), 1.35$ $(\mathrm{s}, 9 \mathrm{H})$.

${ }^{13}$ C NMR $\left(100.6 \mathrm{MHz}, \mathrm{CDCl}_{3}\right) \delta 208.3,141.4,135.4,128.7$ (2C), 128.3 (2C), 44.0, 28.1 (3C), 21.4.

The ${ }^{1} \mathrm{H}$ and ${ }^{13} \mathrm{C}$ NMR spectra data of product 4 aa were consistent with the literature. ${ }^{12}$

\section{1-([1,1'-Biphenyl]-4-yl)-2,2-dimethylpropan-1-one (4ab)}<smiles>CC(C)(C)C(=O)c1ccc(-c2ccccc2)cc1</smiles>

The product 4ab was purified by flash chromatography on silica gel $\left(100: 0-95: 5\right.$, hexane/ $\left.\mathrm{Et}_{2} \mathrm{O}\right)$ (Figure 2; $14.8 \mathrm{mg}, 0.062 \mathrm{mmol}, 62 \%$ isolated yield).

${ }^{1} \mathbf{H}$ NMR $\left(400 \mathrm{MHz}, \mathrm{CDCl}_{3}\right) \delta 7.82(\mathrm{~d}, J=7.6 \mathrm{~Hz}, 2 \mathrm{H}), 7.63-7.61(\mathrm{~m}, 4 \mathrm{H}), 7.46(\mathrm{t}, J=7.2 \mathrm{~Hz}$, 2H), $7.38(\mathrm{~m}, 1 \mathrm{H}), 1.39(\mathrm{~s}, 9 \mathrm{H})$.

${ }^{13}$ C NMR (100.6 MHz, $\left.\mathrm{CDCl}_{3}\right) \delta 208.3,143.7,140.0,136.9,128.9$ (2C), 128.7 (2C), 127.9, 127.1 (2C), 126.7 (2C), 44.2, 28.1 (3C).

The ${ }^{1} \mathrm{H}$ and ${ }^{13} \mathrm{C}$ NMR spectra data of product $4 \mathbf{a b}$ were consistent with the literature. ${ }^{13}$

\section{1-([1,1'-Biphenyl]-4-yl)-2,2,3-trimethylbutan-1-one (4bb)}

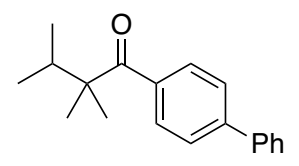

The product $\mathbf{4 b b}$ was purified by flash chromatography on silica gel (100:0-80:20, hexane/Et $\left.{ }_{2} \mathrm{O}\right)$ (Figure 2; $7.3 \mathrm{mg}, 0.027 \mathrm{mmol}, 27 \%$ isolated yield). Yellow solid.

M.p. $64-66^{\circ} \mathrm{C}$.

IR (neat) 669, 751, 970, 1172, 1260, 1395, 1464, 1603, 1670, 2360, $2966 \mathrm{~cm}^{-1}$.

${ }^{1} \mathbf{H}$ NMR $\left(400 \mathrm{MHz}, \mathrm{CDCl}_{3}\right) \delta 7.79(\mathrm{~d}, J=7.6 \mathrm{~Hz}, 2 \mathrm{H}), 7.61(\mathrm{~d}, J=7.6 \mathrm{~Hz}, 4 \mathrm{H}), 7.46(\mathrm{t}, J=7.6$, $2 \mathrm{H}), 7.38(\mathrm{~m}, 1 \mathrm{H}), 2.42(\mathrm{~m}, 1 \mathrm{H}), 1.26(\mathrm{~s}, 6 \mathrm{H}), 0.90(\mathrm{~d}, J=6.4 \mathrm{~Hz}, 6 \mathrm{H})$.

${ }^{13}$ C NMR (100.6 MHz, $\left.\mathrm{CDCl}_{3}\right) \delta 209.2,143.5,140.1,137.9,128.9$ (2C), 128.5 (2C), 127.9, 127.2 (2C), 126.7 (2C), 51.3, 34.1, 22.0 (2C), 17.7 (2C).

HRMS-DART $(\mathrm{m} / \mathrm{z}):[\mathrm{M}+\mathrm{H}]^{+}$calcd for $\mathrm{C}_{19} \mathrm{H}_{23} \mathrm{O}^{+}, 267.1743$; found, 267.1742. 


\section{[1,1'-Biphenyl]-4-yl(cyclohexyl)methanone (4cb)}<smiles>O=C(c1ccc(-c2ccccc2)cc1)C1CCCCC1</smiles>

The product 4cb was purified by flash chromatography on silica gel (100:0-95:5, hexane/Et $2 \mathrm{O})$ (Figure 2; $13.8 \mathrm{mg}, 0.052 \mathrm{mmol}, 52 \%$ isolated yield).

${ }^{1} \mathbf{H}$ NMR $\left(400 \mathrm{MHz}, \mathrm{CDCl}_{3}\right) \delta 8.02(\mathrm{~d}, J=7.6 \mathrm{~Hz}, 2 \mathrm{H}), 7.68(\mathrm{~d}, J=7.6 \mathrm{~Hz}, 2 \mathrm{H}), 7.63(\mathrm{~d}, J=7.2$ $\mathrm{Hz}, 2 \mathrm{H}), 7.47$ (t, $J=7.2 \mathrm{~Hz}, 2 \mathrm{H}), 7.40(\mathrm{~m}, 1 \mathrm{H}), 3.30(\mathrm{~m}, 1 \mathrm{H}), 1.94-1.85(\mathrm{~m}, 4 \mathrm{H}), 1.76(\mathrm{~m}, 1 \mathrm{H}), 1.55-$ $1.26(\mathrm{~m}, 5 \mathrm{H})$.

${ }^{13}$ C NMR (100.6 MHz, $\left.\mathrm{CDCl}_{3}\right) \delta 203.5,145.4,140.0,135.0,128.93$ (2C), 128.86 (2C), 128.1, $127.2(2 \mathrm{C} \times 2), 45.7,29.5(2 \mathrm{C}), 26.0,25.9(2 \mathrm{C})$.

The ${ }^{1} \mathrm{H}$ and ${ }^{13} \mathrm{C}$ NMR spectra data of product $4 \mathbf{c b}$ were consistent with the literature. ${ }^{14}$

1-([1,1'-Biphenyl]-4-yl)-2-methylbutan-1-one (4db)

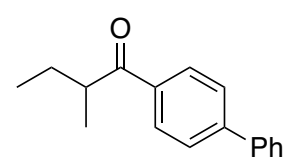

The product $4 \mathbf{d b}$ was purified by flash chromatography on silica gel (100:0-98:2, hexane/Et $2 \mathrm{O})$ (Figure 2; $10.1 \mathrm{mg}, 0.042 \mathrm{mmol}, 42 \%$ isolated yield).

${ }^{1} \mathbf{H}$ NMR $\left(400 \mathrm{MHz}, \mathrm{CDCl}_{3}\right) \delta 8.04(\mathrm{~d}, J=8.0 \mathrm{~Hz}, 2 \mathrm{H}), 7.69(\mathrm{~d}, J=8.0 \mathrm{~Hz}, 2 \mathrm{H}), 7.63(\mathrm{~d}, J=7.2$ $\mathrm{Hz}, 2 \mathrm{H}), 7.47$ (t, $J=7.2 \mathrm{~Hz}, 2 \mathrm{H}), 7.40(\mathrm{~m}, 1 \mathrm{H}), 3.44(\mathrm{~m}, 1 \mathrm{H}), 1.87(\mathrm{~m}, 1 \mathrm{H}), 1.52(\mathrm{~m}, 1 \mathrm{H}), 1.22(\mathrm{~d}, J$ $=6.8 \mathrm{~Hz}, 3 \mathrm{H}), 0.94(\mathrm{t}, J=7.2 \mathrm{~Hz}, 3 \mathrm{H})$.

${ }^{13}$ C NMR (100.6 MHz, $\left.\mathrm{CDCl}_{3}\right) \delta 204.0,145.5,139.9,135.5,128.9$ (2C), $128.8(2 \mathrm{C}), 128.1,127.2$ $(2 \mathrm{C} \times 2), 42.1,26.7,16.8,11.8$.

The ${ }^{1} \mathrm{H}$ and ${ }^{13} \mathrm{C}$ NMR spectra data of product $\mathbf{4 d \mathbf { d b }}$ were consistent with the literature. ${ }^{15}$

\section{1-([1,1'-Biphenyl]-4-yl)pentan-1-one (4eb)}

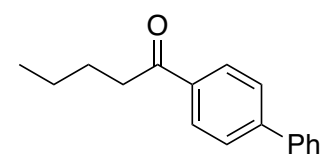

The product 4eb was purified by flash chromatography on silica gel (100:0-95:5, hexane/Et $2 \mathrm{O})$ (Figure 2; $10.5 \mathrm{mg}, 0.044 \mathrm{mmol}, 44 \%$ isolated yield).

${ }^{1} \mathbf{H}$ NMR $\left(400 \mathrm{MHz}, \mathrm{CDCl}_{3}\right) \delta 8.04(\mathrm{~d}, J=7.6 \mathrm{~Hz}, 2 \mathrm{H}), 7.68(\mathrm{~d}, J=7.6 \mathrm{~Hz}, 2 \mathrm{H}), 7.63(\mathrm{~d}, J=7.2$ $\mathrm{Hz}, 2 \mathrm{H}), 7.47(\mathrm{t}, J=7.2 \mathrm{~Hz}, 2 \mathrm{H}), 7.40(\mathrm{~m}, 1 \mathrm{H}), 3.00(\mathrm{t}, J=7.2 \mathrm{~Hz}, 2 \mathrm{H}), 1.75(\mathrm{~m}, 2 \mathrm{H}), 1.48-1.41$ (m, 2H), 0.97 (t, $J=7.2 \mathrm{~Hz}, 3 \mathrm{H})$.

${ }^{13} \mathbf{C}$ NMR (100.6 MHz, $\left.\mathrm{CDCl}_{3}\right) \delta 200.2,145.5,139.9,135.8,128.9$ (2C), 128.7 (2C), 128.2, 127.3 (2C), 127.2 (2C), 38.4, 26.6, 22.5, 14.0 .

The ${ }^{1} \mathrm{H}$ and ${ }^{13} \mathrm{C}$ NMR spectra data of product $4 \mathbf{e b}$ were consistent with the literature. ${ }^{16}$ 


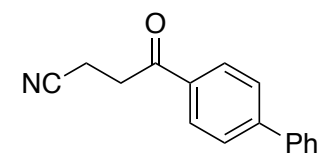

The product $\mathbf{4 f b}$ was purified by flash chromatography on silica gel (100:0-80:20, hexane/AcOEt) (Figure 2; $6.1 \mathrm{mg}, 0.026 \mathrm{mmol}, 26 \%$ isolated yield).

${ }^{1} \mathbf{H}$ NMR $\left(400 \mathrm{MHz}, \mathrm{CDCl}_{3}\right) \delta 8.03(\mathrm{~d}, J=8.0 \mathrm{~Hz}, 2 \mathrm{H}), 7.72(\mathrm{~d}, J=8.0 \mathrm{~Hz}, 2 \mathrm{H}), 7.64(\mathrm{~d}, J=7.2$ $\mathrm{Hz}, 2 \mathrm{H}), 7.49$ (t, $J=7.2 \mathrm{~Hz}, 2 \mathrm{H}), 7.42(\mathrm{~m}, 1 \mathrm{H}), 3.42(\mathrm{t}, J=7.2 \mathrm{~Hz}, 2 \mathrm{H}), 2.81(\mathrm{t}, J=7.2 \mathrm{~Hz}, 2 \mathrm{H})$.

${ }^{13}$ C NMR (100.6 MHz, $\left.\mathrm{CDCl}_{3}\right) \delta 194.9,146.6,139.5,134.3,129.0$ (2C), 128.6 (2C), 128.5, 127.5 (2C), 127.3 (2C), 119.2, 34.3, 11.8.

The ${ }^{1} \mathrm{H}$ and ${ }^{13} \mathrm{C}$ NMR spectra data of product $\mathbf{4 f b}$ were consistent with the literature. ${ }^{17}$

\section{1-([1,1'-Biphenyl]-4-yl)ethan-1-one (4gb)}<smiles>CC(=O)c1ccc(-c2ccccc2)cc1</smiles>

The product 4 gb was purified by flash chromatography on silica gel (100:0-95:5, hexane/Et $\left.{ }_{2} \mathrm{O}\right)$ (Figure 2; $5.0 \mathrm{mg}, 0.026 \mathrm{mmol}, 26 \%$ isolated yield).

${ }^{1} \mathbf{H}$ NMR $\left(400 \mathrm{MHz}, \mathrm{CDCl}_{3}\right) \delta 8.04(\mathrm{~d}, J=8.0 \mathrm{~Hz}, 2 \mathrm{H}), 7.69(\mathrm{~d}, J=8.0 \mathrm{~Hz}, 2 \mathrm{H}), 7.63(\mathrm{~d}, J=7.6$ $\mathrm{Hz}, 2 \mathrm{H}), 7.43$ (t, $J=7.6 \mathrm{~Hz}, 2 \mathrm{H}), 7.41(\mathrm{~m}, 1 \mathrm{H}), 2.65$ (s, 3H).

${ }^{13}$ C NMR (100.6 MHz, $\left.\mathrm{CDCl}_{3}\right) \delta 197.8,145.8,139.9,135.8,128.95$ (2C), 128.91 (2C), 128.2, 127.3 (2C), $127.2(2 \mathrm{C}), 26.7$.

The ${ }^{1} \mathrm{H}$ and ${ }^{13} \mathrm{C}$ NMR spectra data of product $\mathbf{4 g b}$ were consistent with the literature. ${ }^{18}$

\section{1-([1,1'-Biphenyl]-4-yl)ethan-1-one-2,2,2-d $d_{3}([2 \mathrm{H}] 4 \mathrm{gb})$}<smiles>O=C([O-])c1ccc(-c2ccccc2)cc1</smiles>

The product $[2 \mathrm{H}] \mathbf{4 g b}$ was purified by flash chromatography on silica gel (100:0-95:5, hexane/Et ${ }_{2} \mathrm{O}$ ) (Figure 2; $5.0 \mathrm{mg}, 0.026 \mathrm{mmol}, 26 \%$ isolated yield, 93\%D). Pale yellow solid.

M.p. $113-115^{\circ} \mathrm{C}$.

IR (neat) 690, 760, 827, 1117, 1269, 1405, 1602, 1677, 2359, $2922 \mathrm{~cm}^{-1}$.

${ }^{1} \mathbf{H}$ NMR $\left(400 \mathrm{MHz}, \mathrm{CDCl}_{3}\right) \delta 8.04(\mathrm{~d}, J=8.0 \mathrm{~Hz}, 2 \mathrm{H}), 7.69(\mathrm{~d}, J=8.0 \mathrm{~Hz}, 2 \mathrm{H}), 7.63(\mathrm{~d}, J=7.2$ $\mathrm{Hz}, 2 \mathrm{H}), 7.48(\mathrm{t}, J=7.2 \mathrm{~Hz}, 2 \mathrm{H}), 7.40(\mathrm{~m}, 1 \mathrm{H})$.

${ }^{2} \mathbf{H}$ NMR (61.4 MHz, $\left.\mathrm{CDCl}_{3}\right) \delta 2.62$.

${ }^{13} \mathrm{C}$ NMR (100.6 MHz, $\left.\mathrm{CDCl}_{3}\right) \delta 197.9,145.8,139.9,135.8,128.94$ (2C), 128.89 (2C), 128.2, $127.3(2 \mathrm{C}), 127.2(2 \mathrm{C}), 29.7(\mathrm{~m})$.

HRMS-DART $(\mathrm{m} / z)$ : $[\mathrm{M}+\mathrm{H}]^{+}$calcd for $\mathrm{C}_{14} \mathrm{H}_{10} \mathrm{D}_{3} \mathrm{O}^{+}, 200.1149$; found, 200.1150.

The ${ }^{1} \mathrm{H}$ and ${ }^{2} \mathrm{H}$ NMR spectra data of product $[2 \mathrm{H}] \mathbf{4 g b}$ were consistent with the literature. ${ }^{19}$ 
1-(1H-Indol-2-yl)ethan-1-one (4gc)

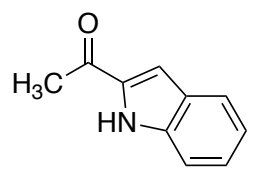

The product 4ge was purified by flash chromatography on silica gel (100:0-80:20, hexane/Et $\left.{ }_{2} \mathrm{O}\right)$ (Figure 2; $2.9 \mathrm{mg}, 0.018 \mathrm{mmol}, 18 \%$ isolated yield).

${ }^{1} \mathbf{H}$ NMR $\left(400 \mathrm{MHz}, \mathrm{CDCl}_{3}\right) \delta 9.00($ br s, $1 \mathrm{H}), 7.72(\mathrm{~d}, J=8.0 \mathrm{~Hz}, 1 \mathrm{H}), 7.42(\mathrm{~d}, J=8.4 \mathrm{~Hz}, 1 \mathrm{H})$, $7.35(\mathrm{t}, J=7.2 \mathrm{~Hz}, 1 \mathrm{H}), 7.21(\mathrm{~s}, 1 \mathrm{H}), 7.16(\mathrm{t}, J=7.2 \mathrm{~Hz}, 1 \mathrm{H}), 2.60(\mathrm{~s}, 3 \mathrm{H})$.

${ }^{13} \mathrm{C}$ NMR (100.6 MHz, $\left.\mathrm{CDCl}_{3}\right) \delta 190.4,137.2,135.4,127.6,126.4,123.1,121.0,112.1,109.8$, 25.8 .

The ${ }^{1} \mathrm{H}$ and ${ }^{13} \mathrm{C}$ NMR spectra data of product $4 \mathrm{gc}$ were consistent with the literature. ${ }^{20}$

\section{1-(1H-Indol-2-yl)ethan-1-one-2,2,2- $d_{3}([2 \mathrm{H}] 4 \mathrm{gc})$}

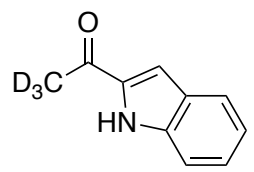

The product $[2 \mathrm{H}] 4$ gc was purified by flash chromatography on silica gel (100:0-80:20, hexane/Et $2 \mathrm{O}$ ) (Figure 2; $3.8 \mathrm{mg}, 0.025 \mathrm{mmol}, 25 \%$ isolated yield, containing 93\%D). Pale yellow solid.

M.p. $140-142^{\circ} \mathrm{C}$.

IR (neat) 692, 754, 813, 1230, 1344, 1523, 1681, 2924, $3335 \mathrm{~cm}^{-1}$.

${ }^{1}$ H NMR $\left(400 \mathrm{MHz}, \mathrm{CDCl}_{3}\right) \delta 8.99($ br s, $1 \mathrm{H}), 7.72(\mathrm{~d}, J=8.0 \mathrm{~Hz}, 1 \mathrm{H}), 7.42(\mathrm{~d}, J=8.4 \mathrm{~Hz}, 1 \mathrm{H})$, $7.35(\mathrm{t}, J=7.2 \mathrm{~Hz}, 1 \mathrm{H}), 7.21(\mathrm{~s}, 1 \mathrm{H}), 7.16(\mathrm{~d}, J=7.2 \mathrm{~Hz}, 1 \mathrm{H})$.

${ }^{2} \mathbf{H}$ NMR (61.4 MHz, $\left.\mathrm{CDCl}_{3}\right) \delta 2.57$.

${ }^{13} \mathrm{C}$ NMR (100.6 MHz, $\left.\mathrm{CDCl}_{3}\right) \delta 190.5,137.2,135.4,127.6,126.4,123.1,121.0,112.1,109.8$, $30.9(\mathrm{~m})$.

HRMS-DART $(\mathrm{m} / \mathrm{z}):[\mathrm{M}]^{+}$calcd for $\mathrm{C}_{10} \mathrm{H}_{6} \mathrm{D}_{3} \mathrm{NO}^{+}, 162.0867$; found, 162.0867 .

\section{1-(4-(4,4,5,5-Tetramethyl-1,3,2-dioxaborolan-2-yl)phenyl)ethan-1-one (4gd)}<smiles>CC(=O)c1ccc(Br)cc1</smiles>

The product 4gd was purified by flash chromatography on silica gel (100:0-90:10, hexane/Et $2 \mathrm{O})$ (Figure 2; $5.3 \mathrm{mg}, 0.022 \mathrm{mmol}, 22 \%$ isolated yield).

${ }^{1} \mathbf{H}$ NMR $\left(400 \mathrm{MHz}, \mathrm{CDCl}_{3}\right) \delta 7.94(\mathrm{~d}, J=8.0 \mathrm{~Hz}, 2 \mathrm{H}), 7.89$ (d, $\left.J=8.0 \mathrm{~Hz}, 2 \mathrm{H}\right), 2.62(\mathrm{~s}, 3 \mathrm{H}), 1.36$ $(\mathrm{s}, 12 \mathrm{H})$. 
${ }^{13}$ C NMR (100.6 MHz, $\mathrm{CDCl}_{3}$ ) $\delta$ 198.5, 139.0, 134.9 (2C), 127.3 (2C), 84.2 (2C), 26.8, 24.9 (4C). A signal connected directly to boron was not observed.

${ }^{11} \mathbf{B}$ NMR (128.4 MHz, $\left.\mathrm{CDCl}_{3}\right) \delta 30.9$.

The ${ }^{1} \mathrm{H},{ }^{13} \mathrm{C}$ and ${ }^{11} \mathrm{~B}$ NMR spectra data of product $4 \mathrm{gd}$ were consistent with the literature. ${ }^{16}$

1-(4-(4,4,5,5-Tetramethyl-1,3,2-dioxaborolan-2-yl)phenyl)ethan-1-one-2,2,2-d $d_{3}([2 \mathrm{H}] 4 \mathrm{gd})$<smiles>O=C([O-])c1ccc(Br)cc1</smiles>

The product $[2 \mathrm{H}] 4 \mathbf{g d}$ was purified by flash chromatography on silica gel (100:0-90:10, hexane/Et $2 \mathrm{O}$ ) (Figure 2; $5.5 \mathrm{mg}, 0.022 \mathrm{mmol}, 22 \%$ isolated yield, containing 93\%D). Orange oil.

IR (neat) 653, 857, 1086, 1144, 1266, 1360, 1398, 1684, 2360, $2979 \mathrm{~cm}^{-1}$.

${ }^{1} \mathbf{H}$ NMR $\left(400 \mathrm{MHz}, \mathrm{CDCl}_{3}\right) \delta 7.94(\mathrm{~d}, J=8.0 \mathrm{~Hz}, 2 \mathrm{H}), 7.89$ (d, $\left.J=8.0 \mathrm{~Hz}, 2 \mathrm{H}\right), 1.36(\mathrm{~s}, 9 \mathrm{H})$.

${ }^{13}$ C NMR (100.6 MHz, $\left.\mathrm{CDCl}_{3}\right) \delta 198.6,139.0,134.9$ (2C), 127.3 (2C), 84.2 (2C), 29.7 (m), 24.9 (4C). A signal connected directly to boron was not observed.

${ }^{11}$ B NMR (128.4 MHz, $\left.\mathrm{CDCl}_{3}\right) \delta 31.1$.

${ }^{2} \mathbf{H}$ NMR (61.4 MHz, $\left.\mathrm{CDCl}_{3}\right) \delta 2.59$.

HRMS-DART $(\mathrm{m} / \mathrm{z}):[\mathrm{M}+\mathrm{H}]^{+}$calcd for $\mathrm{C}_{14} \mathrm{H}_{17} \mathrm{D}_{3} \mathrm{BO}_{3}{ }^{+}, 250.1688$; found, 250.1683.

\section{1-(4-Methoxyphenyl)-2,2-dimethylpropan-1-one (4ae)}

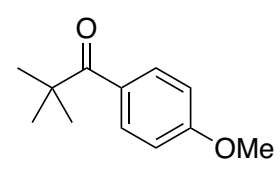

The product 4ae was purified by flash chromatography on silica gel (100:0-90:10, hexane/Et $2 \mathrm{O})$ (Figure 2; $8.8 \mathrm{mg}, 0.046 \mathrm{mmol}, 46 \%$ isolated yield).

${ }^{1} \mathbf{H}$ NMR $\left(400 \mathrm{MHz}, \mathrm{CDCl}_{3}\right) \delta 7.85(\mathrm{~d}, J=8.0 \mathrm{~Hz}, 2 \mathrm{H}), 6.90(\mathrm{~d}, J=8.0 \mathrm{~Hz}, 2 \mathrm{H}), 3.85(\mathrm{~s}, 3 \mathrm{H}), 1.37$ $(\mathrm{s}, 9 \mathrm{H})$.

${ }^{13}$ C NMR (100.6 MHz, $\left.\mathrm{CDCl}_{3}\right) \delta 206.3,162.0,130.9$ (2C), 130.1, 113.2 (2C), 55.3, 43.9, 28.4 (3C).

The ${ }^{1} \mathrm{H}$ and ${ }^{13} \mathrm{C}$ NMR spectra data of product 4ae were consistent with the literature. ${ }^{12}$

\section{1-(3,5-Dimethylphenyl)-2,2-dimethylpropan-1-one (4af)}<smiles>Cc1cc(C)cc(C(=O)C(C)(C)C)c1</smiles>

The product 4af was purified by flash chromatography on silica gel $(100: 0-98: 2$, hexane/Et $2 \mathrm{O})$ (Figure 2; $11.0 \mathrm{mg}, 0.058 \mathrm{mmol}, 58 \%$ isolated yield).

${ }^{1} \mathbf{H}$ NMR (400 MHz, $\left.\mathrm{CDCl}_{3}\right) \delta 7.24(\mathrm{~s}, 2 \mathrm{H}), 7.08(\mathrm{~s}, 1 \mathrm{H}), 2.34(\mathrm{~s}, 6 \mathrm{H}), 1.33(\mathrm{~s}, 9 \mathrm{H})$. 
${ }^{13} \mathrm{C}$ NMR (100.6 MHz, $\left.\mathrm{CDCl}_{3}\right) \delta 210.1,139.0,137.6,132.2$ (2C), 125.3 (2C), 44.2, 28.0 (3C), $21.3(2 \mathrm{C})$.

The ${ }^{1} \mathrm{H}$ and ${ }^{13} \mathrm{C}$ NMR spectra data of product 4af were consistent with the literature. ${ }^{21}$

\section{2,2-Dimethyl-1-(4-(4,4,5,5-tetramethyl-1,3,2-dioxaborolan-2-yl)phenyl)propan-1-one (4ad)}

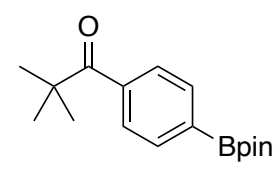

The product 4ad was purified by flash chromatography on silica gel (100:0-90:10, hexane/Et $\left.{ }_{2} \mathrm{O}\right)$ (Figure 2; $13.8 \mathrm{mg}, 0.048 \mathrm{mmol}, 48 \%$ isolated yield). Pale yellow solid.

M.p. $148-151{ }^{\circ} \mathrm{C}$.

IR (neat) 661, 858, 955, 1087, 1143, 1326, 1357, 1396, 1685, 2359, $2972 \mathrm{~cm}^{-1}$.

${ }^{1} \mathbf{H}$ NMR $\left(400 \mathrm{MHz}, \mathrm{CDCl}_{3}\right) \delta 7.83(\mathrm{~d}, J=7.6 \mathrm{~Hz}, 2 \mathrm{H}), 7.61(\mathrm{~d}, J=7.6 \mathrm{~Hz}, 2 \mathrm{H}), 1.35(\mathrm{~s}, 12 \mathrm{H})$, $1.33(\mathrm{~s}, 9 \mathrm{H})$.

${ }^{13} \mathrm{C}$ NMR (100.6 MHz, $\left.\mathrm{CDCl}_{3}\right) \delta 209.9,141.2,134.4$ (2C), 126.6 (2C), 84.1 (2C), 44.2, 27.8 (3C), 24.9 (4C). A signal connected directly to boron was not observed.

${ }^{11}$ B NMR $\left(128.4 \mathrm{MHz}, \mathrm{CDCl}_{3}\right) \delta 30.3$.

HRMS-DART $(\mathrm{m} / \mathrm{z})$ : [M] $]^{+}$calcd for $\mathrm{C}_{17} \mathrm{H}_{25} \mathrm{BO}_{3}{ }^{+}$, 288.1891; found, 288.1893.

\section{1-(4-Chlorophenyl)-2,2-dimethylpropan-1-one (4ag)}<smiles>CC(C)(C)C(=O)c1ccc(Cl)cc1</smiles>

The product 4ag was purified by flash chromatography on silica gel (100:0-98:2, hexane/Et $2 \mathrm{O})$ (Figure 2; $9.5 \mathrm{mg}, 0.048 \mathrm{mmol}, 48 \%$ isolated yield).

${ }^{1} \mathbf{H}$ NMR $\left(400 \mathrm{MHz}, \mathrm{CDCl}_{3}\right) \delta 7.67(\mathrm{~d}, J=8.0 \mathrm{~Hz}, 2 \mathrm{H}), 7.38(\mathrm{~d}, J=8.0 \mathrm{~Hz}, 2 \mathrm{H}), 1.34(\mathrm{~s}, 9 \mathrm{H})$.

${ }^{13}$ C NMR (100.6 MHz, $\mathrm{CDCl}_{3}$ ) $\delta 207.7,137.2,136.5,129.5$ (2C), 128.3 (2C), 44.2, 28.0 (3C).

The ${ }^{1} \mathrm{H}$ and ${ }^{13} \mathrm{C}$ NMR spectra data of product 4 ag were consistent with the literature. ${ }^{12}$

\section{1-(3-Bromophenyl)-2,2-dimethylpropan-1-one (4ah)}<smiles>CC(C)(C)C(=O)c1cccc(Br)c1</smiles>

The product 4ah was purified by flash chromatography on silica gel (100:0-98:2, hexane/Et $2 \mathrm{O})$ (Figure 2; $9.6 \mathrm{mg}, 0.040 \mathrm{mmol}, 40 \%$ isolated yield). Orange oil.

IR (neat) 722, 750, 977, 1181, 1279, 1476, 1562, 1677, $2968 \mathrm{~cm}^{-1}$.

${ }^{1} \mathbf{H}$ NMR $\left(400 \mathrm{MHz}, \mathrm{CDCl}_{3}\right) \delta 7.78(\mathrm{~s}, 1 \mathrm{H}), 7.59(\mathrm{~d}, J=8.0 \mathrm{~Hz}, 2 \mathrm{H}), 7.28(\mathrm{t}, J=8.0 \mathrm{~Hz}, 1 \mathrm{H}), 1.37$ $(\mathrm{s}, 9 \mathrm{H})$. 
${ }^{13}$ C NMR (100.6 MHz, $\left.\mathrm{CDCl}_{3}\right) \delta 207.9,140.5,133.7,130.8,129.6,126.2,122.4,44.3,27.8$ (3C).

HRMS-DART $(\mathrm{m} / z)$ : $[\mathrm{M}+\mathrm{H}]^{+}$calcd for $\mathrm{C}_{11} \mathrm{H}_{14} \mathrm{BrO}^{+}, 241.0223$; found, 241.0223 .

4,4-Dimethyl-1-phenylpentan-3-one (4ai)

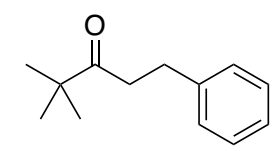

The product 4ai was purified by flash chromatography on silica gel (100:0-98:2, hexane/Et $2 \mathrm{O})$ (Figure 2; $5.5 \mathrm{mg}, 0.029 \mathrm{mmol}, 29 \%$ isolated yield).

${ }^{1} \mathbf{H}$ NMR (400 MHz, $\left.\mathrm{CDCl}_{3}\right) \delta 7.29-7.26(\mathrm{~m}, 2 \mathrm{H}), 7.20-7.18(\mathrm{~m}, 3 \mathrm{H}), 2.88(\mathrm{~d}, J=7.2 \mathrm{~Hz}, 2 \mathrm{H})$, $2.79(\mathrm{~d}, J=7.2 \mathrm{~Hz}, 2 \mathrm{H}), 1.11(\mathrm{~s}, 9 \mathrm{H})$.

${ }^{13} \mathrm{C}$ NMR (100.6 MHz, $\left.\mathrm{CDCl}_{3}\right) \delta 214.9,141.6,128.41$ (2C), 128.38, 126.0 (2C), 44.1, 38.5, 30.1, $26.3(3 \mathrm{C})$.

The ${ }^{1} \mathrm{H}$ and ${ }^{13} \mathrm{C}$ NMR spectra data of product 4ai were consistent with the literature. ${ }^{22}$ 


\section{Optimization of Vicinal Alkylacylation of Alkenes}

Table S4. Optimization of reaction conditions.

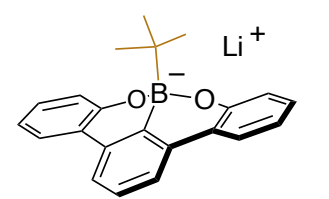

1a

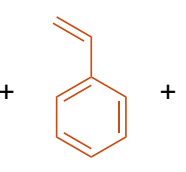

5 a<smiles>O=C(c1ccccc1)n1ccnc1</smiles>

3j

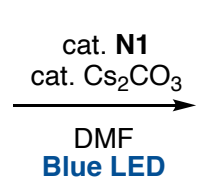

Blue LED

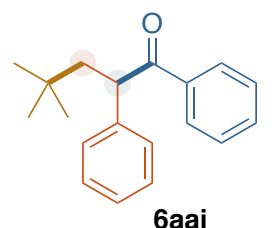

6aaj

\begin{tabular}{|c|c|c|}
\hline & deviation from standard conditions $\mathrm{s}^{\mathrm{a}}$ & Yield (\%) \\
\hline 1 & none (Scheme 1) & 51 \\
\hline 2 & $\mathbf{N} 2$ instead of $\mathbf{N} 1$ & 29 \\
\hline 3 & N3 instead of N1 & 14 \\
\hline 4 & N4 instead of N1 & 17 \\
\hline 5 & N5 instead of N1 & 39 \\
\hline 6 & N7 instead of N1 & 33 \\
\hline $7^{\mathrm{b}}$ & N8 instead of N1 & 25 \\
\hline 8 & N9 instead of N1 & 12 \\
\hline 9 & DMSO instead of DMF & 13 \\
\hline 10 & DMA instead of DMF & 41 \\
\hline 11 & $\operatorname{DMF}(0.33 \mathrm{M})$ & 50 \\
\hline $12^{b}$ & $\mathbf{2 a}$ instead of $\mathbf{1 a}$ & 45 \\
\hline $13^{\mathrm{c}}$ & 2a instead of 1a & 68 \\
\hline $14^{\mathrm{d}}$ & 2a instead of $1 \mathbf{a}$ & 79 \\
\hline
\end{tabular}

${ }^{a}$ Reaction was carried out with 1a $(0.12 \mathrm{mmol}), 3 \mathbf{j}(0.1 \mathrm{mmol})$, styrene $(0.2 \mathrm{mmol})$, catalyst $(0.015 \mathrm{mmol})$ in DMF $(1$ $\mathrm{mL}$ ) under Kessil lamp (440 nm) irradiation equiped with PhotoRedOx Duo for $14 \mathrm{~h} .{ }^{b}$ Reaction was carried out in DMF (300 $\mu \mathrm{L})$ for $6 \mathrm{~h}^{c}{ }^{c}$ Reaction was carried out with $\mathbf{2 a}(0.12 \mathrm{mmol})$, styrene (5a) $(0.2 \mathrm{mmol}), \mathbf{3} \mathbf{j}(0.10 \mathrm{mmol}), \mathbf{N} \mathbf{1}$ catalyst $(0.015 \mathrm{mmol})$ in $\mathrm{MeCN}(300 \mu \mathrm{L})$ under blue LED $(440 \mathrm{~nm})$ irradiation for $6 \mathrm{~h}^{d} 5$ equiv of styrene was used.

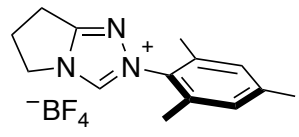

N1

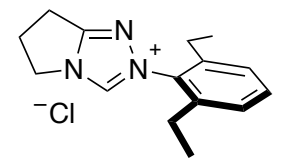

N5

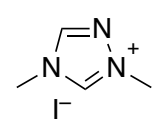

N2

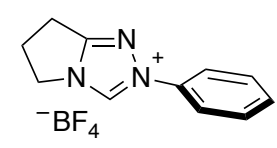

N7

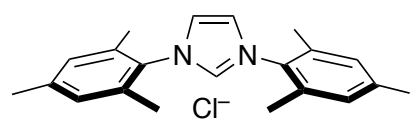

N3

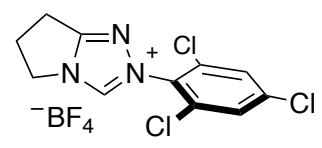

N8
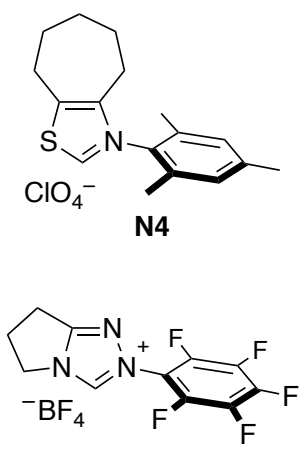

N9 


\section{General Procedure for Three-Component Coupling}

(1)

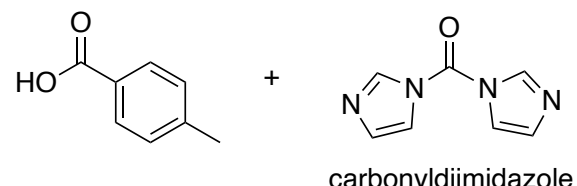

(CDI)
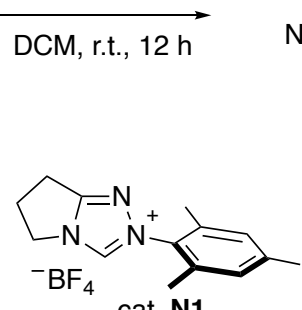

(2)

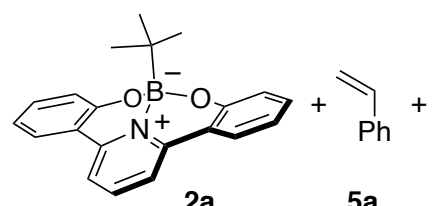

2a

$5 a$<smiles>Cc1ccc(C(=O)n2ccnc2)cc1</smiles>

3a<smiles>Cc1ccc(C(=O)n2ccnc2)cc1</smiles>

3a

(The reaction to produce 6aaa in Figure 3 is representative.) To a suspension of 4-toluic acid $(27.2 \mathrm{mg}, 0.2 \mathrm{mmol})$ in dry dichloromethane $(667 \mu \mathrm{L})$ was added slowly carbonyldiimidazole (CDI, $48.6 \mathrm{mg}, 0.30 \mathrm{mmol}, 1.5 \mathrm{equiv}$ ) (caution, exothermic). After stirring for $12 \mathrm{~h}$ at room temperature, the resulting solution was transferred to separatory funnel and washed with deionized water (ca. 5 $\mathrm{mL}$ ). The organic extract was dried over $\mathrm{Na}_{2} \mathrm{SO}_{4}$ and after filtration, the filtrate was concentrated under reduced pressure to afford the acyl imidazole 3a. The acyl imidazole was used without further purification.

Triazolium salt N1 $(9.5 \mathrm{mg}, 30 \mu \mathrm{mol})$, borate $\mathbf{2 a}(79.0 \mathrm{mg}, 0.24 \mathrm{mmol}), \mathrm{Cs}_{2} \mathrm{CO}_{3}(9.8 \mathrm{mg}, 30 \mu \mathrm{mol})$, and acyl imidazole 3a $(37.2 \mathrm{mg}, 0.2 \mathrm{mmol})$ were placed in an oven-dried screw-top $5 \mathrm{~mL}$ vial containing a magnetic stirring bar. The vial was sealed by a hole cap with rubber septum, and then sparging with nitrogen, followed by addition of alkene $5 \mathbf{a}(46.0 \mu \mathrm{L}, 41.7 \mathrm{mg}, 0.4 \mathrm{mmol})$ and degassed MeCN $(600 \mu \mathrm{L})$. After $6 \mathrm{~h}$ stirring at ambient temperature under photoirradiation $(440 \mathrm{~nm})$, the reaction mixture was evaporated under reduced pressure. After the volatiles were removed under reduced pressure, flash column chromatography on silica gel (100:0-96.5:3.5, hexane/Et $\left.{ }_{2} \mathrm{O}\right)$ gave 6aaa ( $42.4 \mathrm{mg}, 0.15 \mathrm{mmol})$ in $76 \%$ yield.
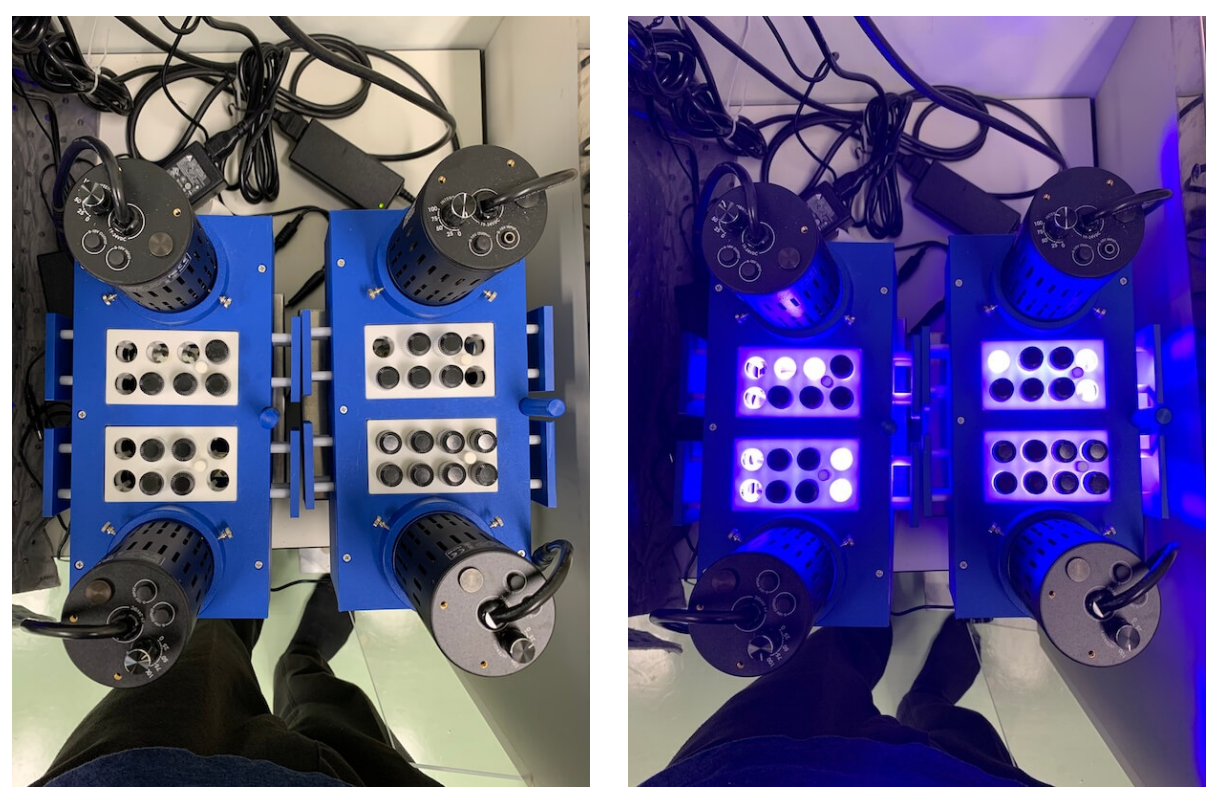

Figure S15. Light set up. 


\section{Characterization Data for Alkylacylation Products}

\section{4,4-Dimethyl-1-(4-methylphenyl)-2-phenylpentan-1-one (6aaa)}

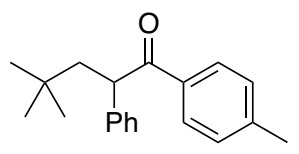

The product 6aaa was purified by flash chromatography on silica gel (100:0-96.5:3.5, hexane/ $\mathrm{Et}_{2} \mathrm{O}$ ) (Figure 3; $42.4 \mathrm{mg}, 0.15 \mathrm{mmol}, 76 \%$ isolated yield, containing $2 \%$ impurity of two component coupling product). White solid.

M.p. $90-93{ }^{\circ} \mathrm{C}$.

IR (neat) 699, 731, 1176, 1222, 1281, 1365, 1456, 1474, 1558, 1606, 1681, $2954 \mathrm{~cm}^{-1}$.

${ }^{1} \mathbf{H}$ NMR $\left(400 \mathrm{MHz}, \mathrm{CDCl}_{3}\right) \delta 7.91(\mathrm{~d}, J=7.6 \mathrm{~Hz}, 2 \mathrm{H}), 7.31(\mathrm{~d}, J=7.6 \mathrm{~Hz}, 2 \mathrm{H}), 7.25(\mathrm{dd}, J=7.2$, $7.2 \mathrm{~Hz}, 2 \mathrm{H}), 7.20(\mathrm{~d}, J=7.2 \mathrm{~Hz}, 2 \mathrm{H}), 7.15(\mathrm{t}, J=7.2 \mathrm{~Hz}, 1 \mathrm{H}), 4.70(\mathrm{~d}, J=9.2 \mathrm{~Hz}, 1 \mathrm{H}), 2.62(\mathrm{dd}, J=$ 14.0, 9.2 Hz, 1H), 2.36 (s, 3H), 1.57 (d, $J=14.0 \mathrm{~Hz}, 1 \mathrm{H}), 0.88$ (s, 9H).

${ }^{13}$ C NMR (100.6 MHz, $\left.\mathrm{CDCl}_{3}\right) \delta 199.5,143.5,141.3,134.4,129.3$ (2C), 128.8 (2C), 128.7 (2C), $128.1(2 \mathrm{C}), 126.6,49.4,47.5,31.2,29.8(3 \mathrm{C}), 21.5$.

HRMS-DART $(\mathrm{m} / z):[\mathrm{M}+\mathrm{H}]^{+}$calcd for $\mathrm{C}_{20} \mathrm{H}_{25} \mathrm{O}^{+}, 281.1900$; found, 281.1898.

\section{4,4,5-Trimethyl-2-phenyl-1-(p-tolyl)hexan-1-one (6baa)}

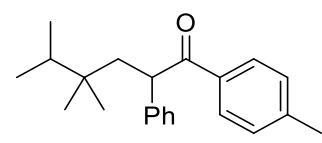

The product 6baa was purified by flash chromatography on silica gel (100:0-85:15, hexane/EtOAc) (Figure 3; $38.7 \mathrm{mg}, 0.13 \mathrm{mmol}, 63 \%$ isolated yield). Colorless oil.

IR (neat) 702, 750, 809, 1175, 1269, 1452, 1605, 1678, 2873, $2959 \mathrm{~cm}^{-1}$.

${ }^{1} \mathbf{H}$ NMR $\left(400 \mathrm{MHz}, \mathrm{CDCl}_{3}\right) \delta 7.91(\mathrm{~d}, J=8.0 \mathrm{~Hz}, 2 \mathrm{H}), 7.33-7.14(\mathrm{~m}, 7 \mathrm{H}), 4.71(\mathrm{~d}, J=8.8 \mathrm{~Hz}$, $1 \mathrm{H}), 2.65$ (dd, $J=14.0,8.8 \mathrm{~Hz}, 1 \mathrm{H}), 2.36(\mathrm{~s}, 3 \mathrm{H}), 1.60-1.48(\mathrm{~m}, 2 \mathrm{H}), 0.85-0.81(\mathrm{~m}, 9 \mathrm{H}), 0.72$ (s, $3 \mathrm{H})$.

${ }^{13} \mathrm{C}$ NMR (100.6 MHz, $\left.\mathrm{CDCl}_{3}\right) \delta 199.6,143.5,141.6,134.5,129.3$ (2C), $128.8(2 \mathrm{C}), 128.7$ (2C), 128.1 (2C), 126.6, 48.7, 43.5, 36.3, 36.0, 24.7, 24.2, 21.5, 17.6, 17.5.

HRMS-DART $(\mathrm{m} / z):[\mathrm{M}+\mathrm{H}]^{+}$calcd for $\mathrm{C}_{22} \mathrm{H}_{29} \mathrm{O}^{+}, 309.2213$; found, 309.2222.

\section{3-((3r,5r,7r)-Adamantan-1-yl)-2-phenyl-1-(p-tolyl)propan-1-one (6haa)}

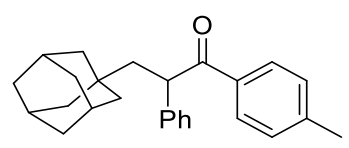

The product 6haa was purified by flash chromatography on silica gel (100:0-85:15, hexane/EtOAc) (Figure 3; $12.1 \mathrm{mg}, 0.034 \mathrm{mmol}, 34 \%$ isolated yield). White solid.

M.p. $98-100^{\circ} \mathrm{C}$. 
IR (neat) 699, 807, 966, 1176, 1232, 1450, 1605, 1679, 2844, $2898 \mathrm{~cm}^{-1}$.

${ }^{1} \mathbf{H}$ NMR $\left(400 \mathrm{MHz}, \mathrm{CDCl}_{3}\right) \delta 7.91(\mathrm{~d}, J=7.6 \mathrm{~Hz}, 2 \mathrm{H}), 7.30-7.13(\mathrm{~m}, 7 \mathrm{H}), 4.75(\mathrm{~d}, J=9.2 \mathrm{~Hz}$, $1 \mathrm{H}), 2.52(\mathrm{dd}, J=13.6,9.2 \mathrm{~Hz}, 1 \mathrm{H}), 2.36(\mathrm{~s}, 3 \mathrm{H}), 1.89(\mathrm{~s}, 3 \mathrm{H}), 1.66-1.53(\mathrm{~m}, 10 \mathrm{H}), 1.42-1.36(\mathrm{~m}$, $3 \mathrm{H})$.

${ }^{13} \mathrm{C}$ NMR (100.6 MHz, $\left.\mathrm{CDCl}_{3}\right) \delta 199.4,143.5,141.5,134.3,129.3(2 \mathrm{C}), 128.8(2 \mathrm{C}+1 \mathrm{C}), 128.1$ (2C), 126.5 (2C), 48.3, 47.3, 42.7 (3C), 36.9 (3C), 33.1, 28.6 (3C), 21.6.

HRMS-DART $(\mathrm{m} / \mathrm{z}):[\mathrm{M}+\mathrm{H}]^{+}$calcd for $\mathrm{C}_{26} \mathrm{H}_{31} \mathrm{O}^{+}, 359.2369$; found, 359.2369.

\section{Ethyl 3,3-Dimethyl-6-oxo-5-phenyl-6-(p-tolyl)hexanoate (6iaa)}

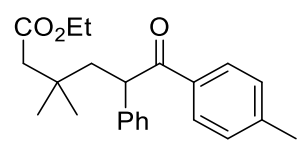

The product 6iaa was purified by flash chromatography on silica gel (100:0-50:50, hexane/EtOAc) (Figure 3; $22.5 \mathrm{mg}, 0.064 \mathrm{mmol}, 64 \%$ isolated yield). Colourless oil.

IR (neat) 700, 1036, 1175, 1224, 1368, 1452, 1605, 1677, 1727, $2959 \mathrm{~cm}^{-1}$.

${ }^{1} \mathbf{H}$ NMR $\left(400 \mathrm{MHz}, \mathrm{CDCl}_{3}\right) \delta 7.91(\mathrm{~d}, J=7.6 \mathrm{~Hz}, 2 \mathrm{H}), 7.33-7.14(\mathrm{~m}, 7 \mathrm{H}), 4.75(\mathrm{~d}, J=8.4 \mathrm{~Hz}$, $1 \mathrm{H}), 4.07(\mathrm{q}, J=6.8 \mathrm{~Hz}, 2 \mathrm{H}), 2.68(\mathrm{dd}, J=14.4,8.4 \mathrm{~Hz}, 1 \mathrm{H}), 2.36(\mathrm{~s}, 3 \mathrm{H}), 2.22(\mathrm{~s}, 2 \mathrm{H}), 1.74(\mathrm{~d}, J=$ $14.4 \mathrm{~Hz}, 1 \mathrm{H}), 1.20(\mathrm{t}, J=6.8 \mathrm{~Hz}, 3 \mathrm{H}), 0.97(\mathrm{~s}, 6 \mathrm{H})$.

${ }^{13}$ C NMR (100.6 MHz, $\left.\mathrm{CDCl}_{3}\right) \delta 199.2,172.0,143.7,140.8,134.2,129.3$ (2C), 128.9 (2C), 128.8 (2C), 128.2 (2C), 126.8, 60.0, 49.0, 46.4, 45.5, 34.0, 27.8, 27.7, 21.6, 14.2.

HRMS-DART $(\mathrm{m} / \mathrm{z}):[\mathrm{M}+\mathrm{H}]^{+}$calcd for $\mathrm{C}_{23} \mathrm{H}_{29} \mathrm{O}_{3}{ }^{+}, 353.2111$; found, 353.2115 .

\section{4,4-Dimethyl-2-phenyl-1-(p-tolyl)heptane-1,6-dione (6jaa)}

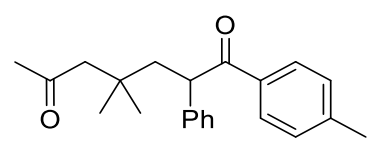

The product 6jaa was purified by flash chromatography on silica gel (100:0-50:50, hexane/EtOAc) (Figure 3; $18.9 \mathrm{mg}, 0.059 \mathrm{mmol}, 59 \%$ isolated yield). Pale yellow solid.

M.p. $71-73{ }^{\circ} \mathrm{C}$.

IR (neat) 701, 733, 974, 1175, 1362, 1605, 1677, 1712, 2871, $2956 \mathrm{~cm}^{-1}$.

${ }^{1} \mathbf{H}$ NMR $\left(400 \mathrm{MHz}, \mathrm{CDCl}_{3}\right) \delta 7.90(\mathrm{~d}, J=7.6 \mathrm{~Hz}, 2 \mathrm{H}), 7.31-7.14(\mathrm{~m}, 7 \mathrm{H}), 4.73(\mathrm{~d}, J=8.8 \mathrm{~Hz}$, 1H), $2.63(\mathrm{dd}, J=14.0,8.8 \mathrm{~Hz}, 1 \mathrm{H}), 2.36(\mathrm{~s}, 3 \mathrm{H}), 2.34$ (d, $J=15.2 \mathrm{~Hz}, 1 \mathrm{H}), 2.23$ (d, $J=15.2 \mathrm{~Hz}$, 1H), 2.05 (s, 3H), 1.77 (d, $J=14.0 \mathrm{~Hz}, 1 \mathrm{H}), 1.01(\mathrm{~s}, 3 \mathrm{H}), 0.98(\mathrm{~s}, 3 \mathrm{H})$.

${ }^{13}$ C NMR (100.6 MHz, $\left.\mathrm{CDCl}_{3}\right) \delta 208.5,199.2,143.7,140.8,134.2,129.3$ (2C), 128.9 (2C), 128.7 (2C), 128.2 (2C), 126.8, 54.3, 49.0, 45.7, 34.1, 32.4, 27.6, 27.5, 21.6.

HRMS-DART $(\mathrm{m} / \mathrm{z}):[\mathrm{M}+\mathrm{H}]^{+}$calcd for $\mathrm{C}_{22} \mathrm{H}_{27} \mathrm{O}_{2}{ }^{+}, 323.2006$; found, 323.2012. 


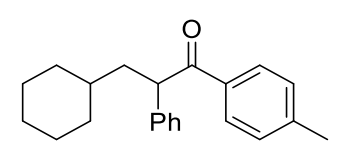

The product 6caa was purified by flash chromatography on silica gel (100:0-85:15, hexane/EtOAc) (Figure 3; $13.9 \mathrm{mg}, 0.045 \mathrm{mmol}, 45 \%$ isolated yield). White solid.

M.p. $89-91{ }^{\circ} \mathrm{C}$.

IR (neat) 700, 739, 940, 1176, 1448, 1493, 1606, 1677, 2850, $2920 \mathrm{~cm}^{-1}$.

${ }^{1} \mathbf{H}$ NMR $\left(400 \mathrm{MHz}, \mathrm{CDCl}_{3}\right) \delta 7.88(\mathrm{~d}, J=7.6 \mathrm{~Hz}, 2 \mathrm{H}), 7.31-7.26(\mathrm{~m}, 4 \mathrm{H}), 7.19(\mathrm{~d}, J=7.6 \mathrm{~Hz}$, $3 \mathrm{H}), 4.69$ (t, $J=7.2 \mathrm{~Hz}, 1 \mathrm{H}), 2.36(\mathrm{~s}, 3 \mathrm{H}), 2.13(\mathrm{~m}, 1 \mathrm{H}), 1.82(\mathrm{~d}, J=12.4 \mathrm{~Hz}, 1 \mathrm{H}), 1.72-1.60$ (m, $5 \mathrm{H}), 1.26-1.06(\mathrm{~m}, 4 \mathrm{H}), 0.95-0.84(\mathrm{~m}, 2 \mathrm{H})$.

${ }^{13}$ C NMR (100.6 MHz, $\left.\mathrm{CDCl}_{3}\right) \delta 199.6,143.6,140.2,134.4,129.2(2 \mathrm{C}), 128.78(2 \mathrm{C}), 128.76(2 \mathrm{C})$, 128.2 (2C), 126.8, 50.3, 41.7, 35.3, 33.6, 33.3, 26.5, 26.14, 26.13, 21.6.

HRMS-DART $(\mathrm{m} / \mathrm{z}):[\mathrm{M}+\mathrm{H}]^{+}$calcd for $\mathrm{C}_{22} \mathrm{H}_{27} \mathrm{O}^{+}, 307.2056$; found, 307.2053.

\section{Ethyl 3-methyl-6-oxo-5-phenyl-6-(p-tolyl)hexanoate (6kaa)}

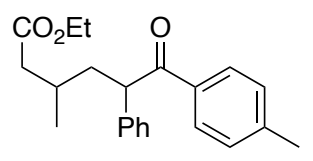

The product 6kaa was purified by flash chromatography on silica gel (100:0-60:40, hexane/EtOAc) (Figure 3; $32.2 \mathrm{mg}, 0.095 \mathrm{mmol}, 48 \%$ isolated yield). The diastereomeric ratio is 1:1 determined by ${ }^{1} \mathrm{H}$ NMR. Pale orange oil.

IR (neat) 701, 741, 1030, 1175, 1265, 1453, 1605, 1676, 1728, $2959 \mathrm{~cm}^{-1}$.

${ }^{1} \mathbf{H}$ NMR $\left(400 \mathrm{MHz}, \mathrm{CDCl}_{3}\right) \delta 7.87(\mathrm{~d}, J=8.0 \mathrm{~Hz}, 2 \mathrm{H}), 7.29-7.26(\mathrm{~m}, 4 \mathrm{H}), 7.19(\mathrm{~d}, J=7.6 \mathrm{~Hz}$, $3 \mathrm{H}), 4.69-4.63(\mathrm{~m}, 1 \mathrm{H}), 4.14-4.03(\mathrm{~m}, 2 \mathrm{H}), 2.35(\mathrm{~s}, 3 \mathrm{H}), 2.40-2.24(\mathrm{~m}, 0.5 \times 3 \mathrm{H}), 2.17-2.12(\mathrm{~m}$, $1 \mathrm{H}), 2.04(\mathrm{~m}, 0.5 \times 1 \mathrm{H}), 1.94-1.86(\mathrm{~m}, 0.5 \times 3 \mathrm{H}), 1.68(\mathrm{~m}, 0.5 \times 1 \mathrm{H}), 1.25-1.18(\mathrm{~m}, 3 \mathrm{H}), 1.00(\mathrm{~d}, J$ $=6.0 \mathrm{~Hz}, 0.5 \times 3 \mathrm{H}), 0.94(\mathrm{~d}, J=7.2 \mathrm{~Hz}, 0.5 \times 3 \mathrm{H})$.

${ }^{13}$ C NMR (100.6 MHz, $\left.\mathrm{CDCl}_{3}\right) \delta 199.1,198.9,172.74,172.68,143.69,143.66,139.8,139.4,134.2$, 134.1, 129.22 (2C), $129.21(2 \mathrm{C}), 128.91(2 \mathrm{C}), 128.89(2 \mathrm{C}), 128.8(2 \mathrm{C} \times 2), 128.2(2 \mathrm{C}), 128.1(2 \mathrm{C})$, $127.00,126.97,60.2,60.1,50.9,50.8,42.0,41.7,40.9,40.4,28.5,28.2,21.6(2 \mathrm{C}), 20.0,19.7,14.23$, 14.19 .

HRMS-DART (m/z): [M+H $]^{+}$calcd for $\mathrm{C}_{22} \mathrm{H}_{27} \mathrm{O}_{3}{ }^{+}, 339.1955$; found, 339.1959.

\section{2-Phenyl-1-(p-tolyl)heptan-1-one (6eaa)}

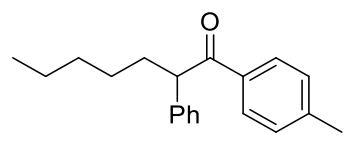

The product 6eaa was purified by flash chromatography on silica gel (100:0-85:15, hexane/EtOAc) (Figure 3; $11.8 \mathrm{mg}, 0.042 \mathrm{mmol}, 42 \%$ isolated yield). Colorless oil. 
IR (neat) 700, 799, 1030, 1176, 1453, 1493, 1606, 1677, 2857, $2926 \mathrm{~cm}^{-1}$.

${ }^{1} \mathbf{H}$ NMR $\left(400 \mathrm{MHz}, \mathrm{CDCl}_{3}\right) \delta 7.87(\mathrm{~d}, J=7.6 \mathrm{~Hz}, 2 \mathrm{H}), 7.31-7.26(\mathrm{~m}, 4 \mathrm{H}), 7.18(\mathrm{~d}, J=6.8 \mathrm{~Hz}$, $3 \mathrm{H}), 4.51(\mathrm{t}, J=7.2 \mathrm{~Hz}, 1 \mathrm{H}), 2.35(\mathrm{~s}, 3 \mathrm{H}), 2.17(\mathrm{~m}, 1 \mathrm{H}), 1.79(\mathrm{~m}, 1 \mathrm{H}), 1.28-1.24(\mathrm{~m}, 6 \mathrm{H}), 0.84-0.83$ $(\mathrm{m}, 3 \mathrm{H})$.

${ }^{13} \mathrm{C}$ NMR (100.6 MHz, $\left.\mathrm{CDCl}_{3}\right) \delta 199.7,143.5,140.1,134.5,129.2(2 \mathrm{C}), 128.8(2 \mathrm{C}+1 \mathrm{C}), 128.2$ (2C), 126.8 (2C), 53.5, 34.0, 31.8, 27.4, 22.5, 21.6, 14.0.

HRMS-DART $(\mathrm{m} / \mathrm{z}):[\mathrm{M}+\mathrm{H}]^{+}$calcd for $\mathrm{C}_{20} \mathrm{H}_{25} \mathrm{O}^{+}, 281.1900$; found, 281.1905.

\section{6-Oxo-5-phenyl-6-(p-tolyl)hexanenitrile (6faa)}

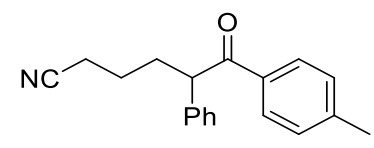

The product 6faa was purified by flash chromatography on silica gel (95:5-60:40, hexane/EtOAc) (Figure 3; $6.9 \mathrm{mg}, 0.025 \mathrm{mmol}, 25 \%$ isolated yield). Pale yellow oil.

IR (neat) 701, 755, 1176, 1230, 1453, 1605, 1675, 2360, 2850, $2921 \mathrm{~cm}^{-1}$.

${ }^{1} \mathbf{H}$ NMR $\left(400 \mathrm{MHz}, \mathrm{CDCl}_{3}\right) \delta 7.84(\mathrm{~d}, J=7.6 \mathrm{~Hz}, 2 \mathrm{H}), 7.32-7.26(\mathrm{~m}, 4 \mathrm{H}), 7.23-7.12(\mathrm{~m}, 3 \mathrm{H})$, $4.53(\mathrm{t}, J=6.8 \mathrm{~Hz}, 1 \mathrm{H}), 2.40-2.24(\mathrm{~m}, 6 \mathrm{H}), 1.99(\mathrm{~m}, 1 \mathrm{H}), 1.73-1.58(\mathrm{~m}, 2 \mathrm{H})$.

${ }^{13}$ C NMR (100.6 MHz, $\left.\mathrm{CDCl}_{3}\right) \delta 198.5,144.0,139.0,133.9,129.3$ (2C), 129.1 (2C), 128.8 (2C), $128.0(2 \mathrm{C}), 127.3,119.4,52.9,32.9,23.6,21.6,17.3$.

HRMS-DART $(\mathrm{m} / \mathrm{z})$ : $[\mathrm{M}+\mathrm{H}]^{+}$calcd for $\mathrm{C}_{19} \mathrm{H}_{20} \mathrm{NO}^{+}, 278.1539$; found, 278.1537 .

\section{2-Phenyl-1-(p-tolyl)butan-1-one (6gaa)}

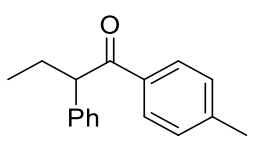

The product 6gaa was purified by flash chromatography on silica gel (100:0-80:20, hexane/EtOAc) (Figure 3; $6.9 \mathrm{mg}, 0.029 \mathrm{mmol}, 29 \%$ isolated yield).

${ }^{1} \mathbf{H}$ NMR (400 MHz, $\left.\mathrm{CDCl}_{3}\right) \delta 7.87(\mathrm{~d}, J=7.6 \mathrm{~Hz}, 2 \mathrm{H}), 7.29-7.25(\mathrm{~m}, 4 \mathrm{H}), 7.18(\mathrm{~d}, J=7.6 \mathrm{~Hz}, 3 \mathrm{H})$, $4.42(\mathrm{t}, J=7.2 \mathrm{~Hz}, 1 \mathrm{H}), 2.35(\mathrm{~s}, 3 \mathrm{H}), 2.19(\mathrm{~m}, 1 \mathrm{H}), 1.85(\mathrm{~m}, 1 \mathrm{H}), 0.90(\mathrm{t}, J=7.2 \mathrm{~Hz}, 3 \mathrm{H})$.

${ }^{13}$ C NMR (100.6 MHz, $\left.\mathrm{CDCl}_{3}\right) \delta 199.7,143.5,139.9,134.5,129.2$ (2C), 128.8 (3C), 128.2 (2C), $126.8(2 \mathrm{C}), 55.3,27.1,21.5,12.3$.

The ${ }^{1} \mathrm{H}$ and ${ }^{13} \mathrm{C}$ NMR spectra data of product 6 gaa was consistent with the literature. ${ }^{23}$

\section{1-(3,5-Dimethylphenyl)-4,4-dimethyl-2-phenylpentan-1-one (6aaf)}<smiles>Cc1cc(C)cc(C(=O)C(CC(C)(C)C)c2ccccc2)c1</smiles> 
The product 6aaf was purified by flash chromatography on silica gel (100:0-85:15, hexane/EtOAc) (Figure 3; $33.3 \mathrm{mg}, 0.11 \mathrm{mmol}, 57 \%$ isolated yield). White solid.

M.p. $31-33{ }^{\circ} \mathrm{C}$.

IR (neat) 700, 832, 1153, 1297, 1452, 1492, 1605, 1681, 2866, $2952 \mathrm{~cm}^{-1}$.

${ }^{1} \mathbf{H}$ NMR $\left(400 \mathrm{MHz}, \mathrm{CDCl}_{3}\right) \delta 7.59(\mathrm{~s}, 2 \mathrm{H}), 7.32-7.24(\mathrm{~m}, 4 \mathrm{H}), 7.18-7.13(\mathrm{~m}, 2 \mathrm{H}), 4.70(\mathrm{dd}, J=$ 8.8, 2.4 Hz, 1H), $2.61(\mathrm{dd}, J=14.0,8.8 \mathrm{~Hz}, 1 \mathrm{H}), 2.33(\mathrm{~s}, 6 \mathrm{H}), 1.56(\mathrm{~m}, 1 \mathrm{H}), 0.89(\mathrm{~s}, 9 \mathrm{H})$.

${ }^{13} \mathrm{C}$ NMR (100.6 MHz, $\left.\mathrm{CDCl}_{3}\right) \delta 200.3,141.3,138.1,137.1,134.5(2 \mathrm{C}), 128.8(2 \mathrm{C}), 128.1(2 \mathrm{C})$, 126.6, 126.4 (2C), 49.6, 47.7, 31.2, 29.8 (3C), 21.3 (2C).

HRMS-DART $(\mathrm{m} / \mathrm{z}):[\mathrm{M}+\mathrm{H}]^{+}$calcd for $\mathrm{C}_{21} \mathrm{H}_{27} \mathrm{O}^{+}$, 295.2056; found, 295.2054.

\section{4,4-dimethyl-1,2-diphenylpentan-1-one (6aaj)}

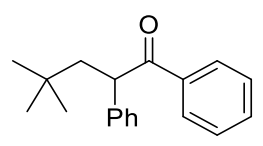

The product 6aaj was purified by flash chromatography on silica gel (100:0-85:15, hexane/EtOAc) (Table 1; $30.0 \mathrm{mg}, 0.11 \mathrm{mmol}, 56 \%$ isolated yield).

${ }^{1} \mathbf{H}$ NMR $\left(400 \mathrm{MHz}, \mathrm{CDCl}_{3}\right) \delta 8.00(\mathrm{~d}, J=7.2 \mathrm{~Hz}, 2 \mathrm{H}), 7.50(\mathrm{~m}, 1 \mathrm{H}), 7.43-7.39(\mathrm{~m}, 2 \mathrm{H}), 7.33-$ $7.25(\mathrm{~m}, 4 \mathrm{H}), 7.17(\mathrm{~m}, 1 \mathrm{H}), 4.72(\mathrm{dd}, J=8.8,3.2 \mathrm{~Hz}, 1 \mathrm{H}), 2.63(\mathrm{dd}, J=14.0,8.8 \mathrm{~Hz}, 1 \mathrm{H}), 1.58$ (dd, $J=14.0,3.2 \mathrm{~Hz}, 1 \mathrm{H}), 0.89(\mathrm{~s}, 9 \mathrm{H})$.

${ }^{13} \mathrm{C}$ NMR (100.6 MHz, $\left.\mathrm{CDCl}_{3}\right) \delta 200.0,141.1,137.0,132.8,128.9$ (2C), $128.6(2 \mathrm{C}), 128.5(2 \mathrm{C})$, 128.1 (2C), 126.7, 49.6, 47.6, 31.2, 29.8 (3C).

The ${ }^{1} \mathrm{H}$ and ${ }^{13} \mathrm{C}$ NMR spectra data of product 6aa was consistent with the literature. ${ }^{24}$

\section{1-(4-Chlorophenyl)-4,4-dimethyl-2-phenylpentan-1-one (6aag)}

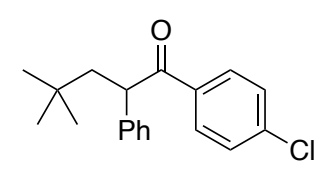

The product 6aag was purified by flash chromatography on silica gel (100:0-90:10, hexane/EtOAc) (Figure 3; $50.4 \mathrm{mg}, 0.164 \mathrm{mmol}, 82 \%$ isolated yield, containing 3\% impurity of two component coupling product). Pale yellow solid.

M.p. $106-109{ }^{\circ} \mathrm{C}$.

IR (neat) 698, 974, 1093, 1217, 1280, 1365, 1399, 1588, 1683, $2954 \mathrm{~cm}^{-1}$.

${ }^{1} \mathbf{H}$ NMR $\left(400 \mathrm{MHz}, \mathrm{CDCl}_{3}\right) \delta 7.93(\mathrm{~d}, J=8.0 \mathrm{~Hz}, 2 \mathrm{H}), 7.38(\mathrm{~d}, J=8.0 \mathrm{~Hz}, 2 \mathrm{H}), 7.28-7.26(\mathrm{~m}$, $4 \mathrm{H}), 7.18(\mathrm{~m}, 1 \mathrm{H}), 4.64(\mathrm{~d}, J=8.8 \mathrm{~Hz}, 1 \mathrm{H}), 2.61(\mathrm{dd}, J=14.0,8.8 \mathrm{~Hz}, 1 \mathrm{H}), 1.57(\mathrm{~d}, J=14.0 \mathrm{~Hz}, 1 \mathrm{H})$, $0.88(\mathrm{~s}, 9 \mathrm{H})$.

${ }^{13} \mathrm{C}$ NMR (100.6 MHz, $\left.\mathrm{CDCl}_{3}\right) \delta 198.7,140.8,139.2,135.3,130.0$ (2C), 129.0 (2C), 128.9 (2C), 128.0 (2C), 126.9, 49.7, 47.4, 31.2, 29.8 (3C).

HRMS-DART $(\mathrm{m} / z)$ : $[\mathrm{M}+\mathrm{H}]^{+}$calcd for $\mathrm{C}_{19} \mathrm{H}_{22} \mathrm{ClO}^{+}, 301.1354$; found, 301.1349 . 


\section{1-(3-Methoxyphenyl)-4,4-dimethyl-2-phenylpentan-1-one (6aak)}

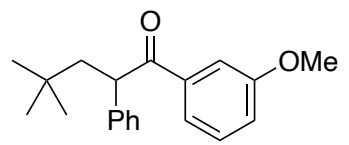

The product 6aak was purified by flash chromatography on silica gel (100:0-95:5, hexane/EtOAc) (Figure 3; $31.5 \mathrm{mg}, 0.102 \mathrm{mmol}, 54 \%$ isolated yield, containing 7\% impurity). Pale yellow solid.

M.p. $65-67^{\circ} \mathrm{C}$.

IR (neat) 700, 729, 774, 1044, 1259, 1429, 1489, 1596, 1684, 2359, $2955 \mathrm{~cm}^{-1}$.

${ }^{1}$ H NMR (400 MHz, $\left.\mathrm{CDCl}_{3}\right) \delta 7.53(\mathrm{~d}, J=7.6 \mathrm{~Hz}, 1 \mathrm{H}), 7.43(\mathrm{~s}, 1 \mathrm{H}), 7.26-7.17(\mathrm{~m}, 5 \mathrm{H}), 7.09$ (t, $J=7.6 \mathrm{~Hz}, 1 \mathrm{H}), 6.96(\mathrm{~d}, J=7.6 \mathrm{~Hz}, 1 \mathrm{H}), 4.62(\mathrm{~d}, J=8.8 \mathrm{~Hz}, 1 \mathrm{H}), 3.74(\mathrm{~s}, 3 \mathrm{H}), 2.55(\mathrm{dd}, J=14.0$, $8.8 \mathrm{~Hz}, 1 \mathrm{H}), 1.50(\mathrm{~d}, J=14.0 \mathrm{~Hz}, 1 \mathrm{H}), 0.81(\mathrm{~s}, 9 \mathrm{H})$.

${ }^{13}$ C NMR (100.6 MHz, $\left.\mathrm{CDCl}_{3}\right) \delta 199.8,159.8,141.1,138.4,129.5,128.9$ (2C), 128.1 (2C), 126.7, 121.1, 119.1, 113.1, 55.3, 49.7, 47.6, 31.2, 29.8 (3C).

HRMS-DART $(\mathrm{m} / \mathrm{z})$ : $[\mathrm{M}+\mathrm{H}]^{+}$calcd for $\mathrm{C}_{20} \mathrm{H}_{25} \mathrm{O}_{2}{ }^{+}, 297.1849$; found, 297.1849.

\section{1-([1,1'-Biphenyl]-4-yl)-4,4-dimethyl-2-phenylpentan-1-one (6aab)}

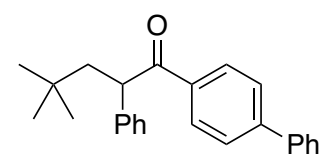

The product 6aab was purified by flash chromatography on silica gel (100:0-98:2, hexane/Et $\left.{ }_{2} \mathrm{O}\right)$ (Figure 3; $41.7 \mathrm{mg}, 0.120 \mathrm{mmol}, 62 \%$ isolated yield, containing 1\% impurity). White solid.

M.p. $138-141^{\circ} \mathrm{C}$.

IR (neat) 697, 719, 734, 762, 1218, 1281, 1365, 1602, 1676, $2953 \mathrm{~cm}^{-1}$.

${ }^{1} \mathbf{H}$ NMR $\left(400 \mathrm{MHz}, \mathrm{CDCl}_{3}\right) \delta 8.08(\mathrm{~d}, J=7.6 \mathrm{~Hz}, 2 \mathrm{H}), 7.63(\mathrm{~d}, J=7.6 \mathrm{~Hz}, 2 \mathrm{H}), 7.58(\mathrm{~d}, J=7.6$ $\mathrm{Hz}, 2 \mathrm{H}), 7.44$ (t, $J=7.2 \mathrm{~Hz}, 2 \mathrm{H}), 7.39-7.34(\mathrm{~m}, 3 \mathrm{H}), 7.30-7.16(\mathrm{~m}, 3 \mathrm{H}), 4.75$ (d, $J=9.2 \mathrm{~Hz}, 1 \mathrm{H})$, $2.66(\mathrm{dd}, J=14.0,9.2 \mathrm{~Hz}, 1 \mathrm{H}), 1.60(\mathrm{~d}, J=14.0 \mathrm{~Hz}, 1 \mathrm{H}), 0.91(\mathrm{~s}, 9 \mathrm{H})$.

${ }^{13} \mathrm{C}$ NMR $\left(100.6 \mathrm{MHz}, \mathrm{CDCl}_{3}\right) \delta 199.5,145.5,141.2,139.9,135.6,129.2(2 \mathrm{C}), 128.9(2 \mathrm{C} \times 2)$, $128.1(2 \mathrm{C}+1 \mathrm{C}), 127.25(2 \mathrm{C}), 127.21(2 \mathrm{C}), 126.7,49.6,47.5,31.2,29.8(3 \mathrm{C})$.

HRMS-DART $(\mathrm{m} / z)$ : $[\mathrm{M}+\mathrm{H}]^{+}$calcd for $\mathrm{C}_{25} \mathrm{H}_{27} \mathrm{O}^{+}, 343.2056$; found, 343.2058 .

\section{4,4-Dimethyl-1-(naphthalen-2-yl)-2-phenylpentan-1-one (6aal)}

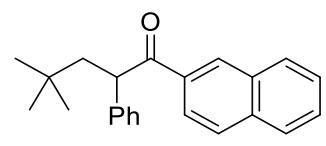

The product 6aal was purified by flash chromatography on silica gel (100:0-95:5, hexane/EtOAc) (Figure 3; $46.9 \mathrm{mg}, 0.15 \mathrm{mmol}, 74 \%$ isolated yield). White solid.

M.p. $106-108^{\circ} \mathrm{C}$.

IR (neat) 698, 726, 821, 1171, 1280, 1393, 1467, 1677, 2953, $3060 \mathrm{~cm}^{-1}$. 
${ }^{1} \mathbf{H}$ NMR $\left(400 \mathrm{MHz}, \mathrm{CDCl}_{3}\right) \delta 8.54(\mathrm{~s}, 1 \mathrm{H}), 8.05(\mathrm{~d}, J=8.4 \mathrm{~Hz}, 1 \mathrm{H}), 7.95(\mathrm{~d}, J=7.6 \mathrm{~Hz}, 1 \mathrm{H})$, $7.84(\mathrm{t}, J=7.2 \mathrm{~Hz}, 2 \mathrm{H}), 7.58-7.50(\mathrm{~m}, 2 \mathrm{H}), 7.38(\mathrm{~d}, J=7.6 \mathrm{~Hz}, 2 \mathrm{H}), 7.29-7.25(\mathrm{~m}, 2 \mathrm{H}), 7.16(\mathrm{~m}$, $1 \mathrm{H}), 4.88(\mathrm{~d}, J=8.8 \mathrm{~Hz}, 1 \mathrm{H}), 2.69(\mathrm{dd}, J=14.0,8.8 \mathrm{~Hz}, 1 \mathrm{H}), 1.63(\mathrm{~d}, J=14.0 \mathrm{~Hz}, 1 \mathrm{H}), 0.92(\mathrm{~s}, 9 \mathrm{H})$. ${ }^{13} \mathbf{C}$ NMR $\left(100.6 \mathrm{MHz}, \mathrm{CDCl}_{3}\right) \delta 199.9,141.2,135.4,134.3,132.5,130.0,129.6,128.9$ (2C), 128.4, $128.3,128.1$ (2C), 127.7, 126.7, 126.6, 124.5, 49.7, 47.6, 31.2, 29.8 (3C).

HRMS-DART $(\mathrm{m} / \mathrm{z}):[\mathrm{M}+\mathrm{H}]^{+}$calcd for $\mathrm{C}_{23} \mathrm{H}_{25} \mathrm{O}^{+}, 317.1900$; found, 317.1901.

\section{4,4-Dimethyl-1-[4-(4,4,5,5-tetramethy-1,3,2-dioxaborolan-2-yl)phenyl]-2-phenylpentan-1-one (6aad)}

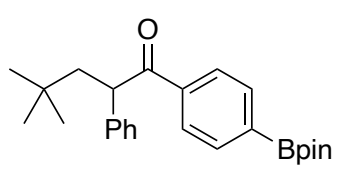

The product 6aad was purified by flash chromatography on silica gel (100:0-90:10, hexane/Et $\left.{ }_{2} \mathrm{O}\right)$ (Figure 3; $34.2 \mathrm{mg}, 0.0872 \mathrm{mmol}, 44 \%$ isolated yield). Pale yellow solid.

M.p. $157-161{ }^{\circ} \mathrm{C}$.

IR (neat) 651, 696, 858, 1090, 1144, 1215, 1364, 1399, 1675, $2950 \mathrm{~cm}^{-1}$.

${ }^{1} \mathbf{H}$ NMR $\left(400 \mathrm{MHz}, \mathrm{CDCl}_{3}\right) \delta 7.96(\mathrm{~d}, J=7.6 \mathrm{~Hz}, 2 \mathrm{H}), 7.84(\mathrm{~d}, J=7.6 \mathrm{~Hz}, 2 \mathrm{H}), 7.31-7.23$ (m, $4 \mathrm{H}), 7.15(\mathrm{t}, J=7.2 \mathrm{~Hz}, 1 \mathrm{H}), 4.72(\mathrm{~d}, J=8.8 \mathrm{~Hz}, 1 \mathrm{H}), 2.62(\mathrm{dd}, J=13.6 \mathrm{~Hz}, 8.8 \mathrm{~Hz}, 1 \mathrm{H}), 1.58(\mathrm{~d}, J$ $=13.6 \mathrm{~Hz}, 1 \mathrm{H}), 1.33(\mathrm{~s}, 12 \mathrm{H}), 0.88(\mathrm{~s}, 9 \mathrm{H})$.

${ }^{13}$ C NMR (100.6 MHz, $\left.\mathrm{CDCl}_{3}\right) \delta 200.3,141.0,138.9,134.9$ (2C), 128.8 (2C), 128.2 (2C), 127.6 (2C), 126.7, 84.1 (2C), 49.7, 47.4, 31.2, 29.8 (3C), 24.84 (2C), 24.82 (2C). A signal connected directly to boron was not observed.

${ }^{11} \mathbf{B}$ NMR $\left(128.4 \mathrm{MHz}, \mathrm{CDCl}_{3}\right) \delta 30.8$.

HRMS-DART $(\mathrm{m} / \mathrm{z})$ : $[\mathrm{M}+\mathrm{H}]^{+}$calcd for $\mathrm{C}_{25} \mathrm{H}_{34} \mathrm{BO}_{3}{ }^{+}, 393.2596$; found, 393.2601 .

\section{1-[4-(trans-4-Ethylcyclohexyl)phenyl]-4,4-dimethyl-2-phenylpentan-1-one (6aam)}

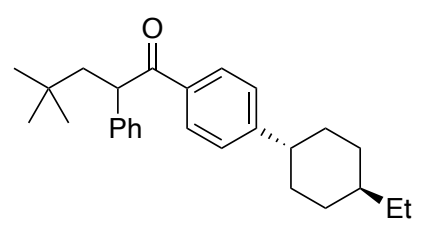

The product 6aam was purified by flash chromatography on silica gel (100:0-98:2, hexane/Et $\left.{ }_{2} \mathrm{O}\right)$ (Figure 3; $47.8 \mathrm{mg}, 0.126 \mathrm{mmol}, 63 \%$ isolated yield). White solid.

M.p. $77-80{ }^{\circ} \mathrm{C}$.

IR (neat) 700, 1176, 1218, 1281, 1365, 1448, 1474, 1605, 1681, 2851, 2922, $2956 \mathrm{~cm}^{-1}$.

${ }^{1} \mathbf{H}$ NMR $\left(400 \mathrm{MHz}, \mathrm{CDCl}_{3}\right) \delta 7.94(\mathrm{~d}, J=7.6 \mathrm{~Hz}, 2 \mathrm{H}), 7.32(\mathrm{~d}, J=7.2 \mathrm{~Hz}, 2 \mathrm{H}), 7.28-7.23$ (m, $4 \mathrm{H}), 7.16(\mathrm{t}, J=7.2 \mathrm{~Hz}, 1 \mathrm{H}), 4.71(\mathrm{~d}, J=8.8 \mathrm{~Hz}, 1 \mathrm{H}), 2.62(\mathrm{dd}, J=13.6,8.8 \mathrm{~Hz}, 1 \mathrm{H}), 2.48(\mathrm{t}, J=$ $11.6 \mathrm{~Hz}, 1 \mathrm{H}), 1.86(\mathrm{~d}, J=10.0 \mathrm{~Hz}, 4 \mathrm{H}), 1.56(\mathrm{~d}, J=13.6 \mathrm{~Hz}, 1 \mathrm{H}), 1.42(\mathrm{q}, J=11.6 \mathrm{~Hz}, 2 \mathrm{H}), 1.25(\mathrm{q}$, $J=7.2 \mathrm{~Hz}, 2 \mathrm{H}), 1.18(\mathrm{~m}, 1 \mathrm{H}), 1.02$ (q, $J=11.6 \mathrm{~Hz}, 2 \mathrm{H}), 0.90$ (t, $J=7.2 \mathrm{~Hz}, 3 \mathrm{H}), 0.88(\mathrm{~s}, 9 \mathrm{H})$. 
${ }^{13}$ C NMR (100.6 MHz, $\left.\mathrm{CDCl}_{3}\right) \delta 199.5,153.2,141.4,134.8,128.82(2 \mathrm{C}), 128.79(2 \mathrm{C}), 128.1$ (2C), 127.1 (2C), 126.6, 49.4, 47.6, 44.7, 39.0, 33.9 (2C), 33.0 (2C), 31.2, 29.9, 29.8 (3C), 11.5.

HRMS-DART (m/z): [M+H] $]^{+}$calcd for $\mathrm{C}_{27} \mathrm{H}_{37} \mathrm{O}^{+}, 377.2839$; found, 377.2842.

\section{4,4-Dimethyl-2-phenyl-1-(pyridin-2-yl)pentan-1-one (6aan)}

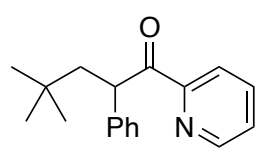

The product 6aan was purified by flash chromatography on silica gel (100:0-95:5, hexane/Et $\left.{ }_{2} \mathrm{O}\right)$ (Figure 3; $27.6 \mathrm{mg}, 0.103 \mathrm{mmol}, 51 \%$ isolated yield, containing trace inpurity of two component coupling product). Pale yellow solid.

M.p. $64-66^{\circ} \mathrm{C}$.

IR (neat) 703, 739, 978, 995, 1218, 1365, 1493, 1582, 1697, $2954 \mathrm{~cm}^{-1}$.

${ }^{1}$ H NMR $\left(400 \mathrm{MHz}, \mathrm{CDCl}_{3}\right) \delta 8.70(\mathrm{~d}, J=4.0 \mathrm{~Hz}, 1 \mathrm{H}), 7.99(\mathrm{~d}, J=8.0 \mathrm{~Hz}, 1 \mathrm{H}), 7.74(\mathrm{dd}, J=8.0$, $8.0 \mathrm{~Hz}, 1 \mathrm{H}), 7.41-7.39(\mathrm{~m}, 3 \mathrm{H}), 7.22(\mathrm{dd}, J=7.2,7.2 \mathrm{~Hz}, 2 \mathrm{H}), 7.13(\mathrm{t}, J=7.2 \mathrm{~Hz}, 1 \mathrm{H}), 5.66(\mathrm{~d}, J=$ $9.2 \mathrm{~Hz}, 1 \mathrm{H}), 2.59$ (dd, $J=13.6,9.2 \mathrm{~Hz}, 1 \mathrm{H}), 1.66(\mathrm{~d}, J=13.6 \mathrm{~Hz}, 1 \mathrm{H}), 0.89$ (s, 9H).

${ }^{13}$ C NMR $\left(100.6 \mathrm{MHz}, \mathrm{CDCl}_{3}\right) \delta 201.5,152.9,148.9,141.0,136.7,128.9$ (2C), $128.4(2 \mathrm{C}), 126.8$, $126.4,122.9,46.9,46.4,31.3,29.8(3 \mathrm{C})$.

HRMS-DART $(\mathrm{m} / \mathrm{z})$ : $[\mathrm{M}+\mathrm{H}]^{+}$calcd for $\mathrm{C}_{18} \mathrm{H}_{22} \mathrm{NO}^{+}$, 268.1696; found, 268.1703.

\section{1-(1H-Indol-2-yl)-4,4-dimethyl-2-phenylpentan-1-one (6aac)}

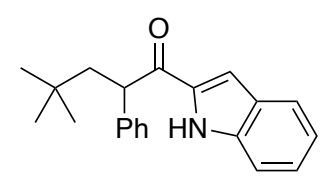

The product 6aac was purified by flash chromatography on silica gel (100:0-88:12, hexane/Et $2 \mathrm{O})$ (Figure 3; $38.8 \mathrm{mg}, 0.127 \mathrm{mmol}, 63 \%$ isolated yield, containing trace impurity of two component coupling product). Pale yellow solid.

M.p. $145-147^{\circ} \mathrm{C}$.

IR (neat) 698, 739, 752, 1137, 1166, 1343, 1520, 1647, 2954, $3317 \mathrm{~cm}^{-1}$.

${ }^{1} \mathbf{H}$ NMR $\left(400 \mathrm{MHz}, \mathrm{CDCl}_{3}\right) \delta 9.04($ br s, $1 \mathrm{H}), 7.71(\mathrm{~d}, J=8.0 \mathrm{~Hz}, 1 \mathrm{H}), 7.40-7.26(\mathrm{~m}, 7 \mathrm{H}), 7.19$ $7.13(\mathrm{~m}, 2 \mathrm{H}), 4.63(\mathrm{~d}, J=9.2 \mathrm{~Hz}, 1 \mathrm{H}), 2.61(\mathrm{dd}, J=13.6,9.2 \mathrm{~Hz}, 1 \mathrm{H}), 1.65(\mathrm{~d}, J=13.6 \mathrm{~Hz}, 1 \mathrm{H})$, $0.92(\mathrm{~s}, 9 \mathrm{H})$.

${ }^{13} \mathrm{C}$ NMR (100.6 MHz, $\left.\mathrm{CDCl}_{3}\right) \delta 192.9,141.2,137.4,134.9,128.8$ (2C), 128.0 (2C), 127.6, 126.8, 126.3, 123.1, 120.9, 112.1, 109.3, 50.2, 47.0, 31.2, 29.7 (3C).

HRMS-DART $(\mathrm{m} / \mathrm{z})$ : $[\mathrm{M}+\mathrm{H}]^{+}$calcd for $\mathrm{C}_{21} \mathrm{H}_{24} \mathrm{NO}^{+}, 306.1852$; found, 306.1854 .

\section{4,4-Dimethyl-2-phenyl-1-(thiophen-3-yl)pentan-1-one (6aao)}




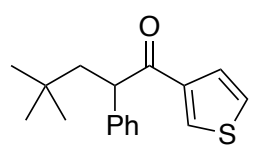

The product 6aao was purified by flash chromatography on silica gel (100:0-97:3, hexane/Et $\left.{ }_{2} \mathrm{O}\right)$ (Figure 3; $36.8 \mathrm{mg}, 0.133 \mathrm{mmol}, 67 \%$ isolated yield, containing 1\% impurity of two component coupling product). White solid.

M.p. $132-134{ }^{\circ} \mathrm{C}$.

IR (neat) 698, 865, 1178, 1226, 1453, 1508, 1660, 2865, 2956, $3092 \mathrm{~cm}^{-1}$.

${ }^{1} \mathbf{H}$ NMR $\left(400 \mathrm{MHz}, \mathrm{CDCl}_{3}\right) \delta 8.10(\mathrm{~s}, 1 \mathrm{H}), 7.56(\mathrm{~d}, J=4.8 \mathrm{~Hz}, 1 \mathrm{H}), 7.32-7.26(\mathrm{~m}, 5 \mathrm{H}), 7.18(\mathrm{t}$, $J=7.2 \mathrm{~Hz}, 1 \mathrm{H}), 4.49(\mathrm{~d}, J=8.8 \mathrm{~Hz}, 1 \mathrm{H}), 2.58(\mathrm{dd}, J=14.0,8.8 \mathrm{~Hz}, 1 \mathrm{H}), 1.56(\mathrm{~d}, J=14.0 \mathrm{~Hz}, 1 \mathrm{H})$, $0.89(\mathrm{~s}, 9 \mathrm{H})$.

${ }^{13} \mathrm{C}$ NMR $\left(100.6 \mathrm{MHz}, \mathrm{CDCl}_{3}\right) \delta 194.2,142.1,141.2,132.2,128.9$ (2C), 128.1 (2C), 127.5, 126.8, 126.2, 51.7, 47.1, 31.1, 29.7 (3C).

HRMS-DART $(\mathrm{m} / \mathrm{z})$ : $[\mathrm{M}+\mathrm{H}]^{+}$calcd for $\mathrm{C}_{17} \mathrm{H}_{21} \mathrm{OS}^{+}, 273.1308$; found, 273.1312 .

\section{1-(Furan-3-yl)-4,4-dimethyl-2-phenylpentan-1-one (6aap)}<smiles>CC(C)(C)CC(C(=O)c1ccoc1)c1ccccc1</smiles>

The product 6aap was purified by flash chromatography on silica gel (100:0-96.5:3.5, hexane/Et $\mathrm{E}_{2} \mathrm{O}$ ) (Figure 3; $31.9 \mathrm{mg}, 0.124 \mathrm{mmol}, 62 \%$ isolated yield). White solid.

M.p. $123-126^{\circ} \mathrm{C}$.

IR (neat) 600, 697, 713, 727, 745, 872, 1154, 1452, 1514, 1666, 2959, $3131 \mathrm{~cm}^{-1}$.

${ }^{1}$ H NMR (400 MHz, $\left.\mathrm{CDCl}_{3}\right) \delta 8.05(\mathrm{~s}, 1 \mathrm{H}), 7.37(\mathrm{~s}, 1 \mathrm{H}), 7.28-7.26(\mathrm{~m}, 4 \mathrm{H}), 7.20(\mathrm{~m}, 1 \mathrm{H}), 6.75$ (s, 1H), $4.22(\mathrm{~d}, J=8.8 \mathrm{~Hz}, 1 \mathrm{H}), 2.56(\mathrm{dd}, J=14.0,8.8 \mathrm{~Hz}, 1 \mathrm{H}), 1.54(\mathrm{~d}, J=14.0 \mathrm{~Hz}, 1 \mathrm{H}), 0.88$ (s, 9H).

${ }^{13} \mathrm{C}$ NMR $\left(100.6 \mathrm{MHz} \mathrm{CDCl}_{3}\right) \delta 194.7,147.1,144.1,141.1,128.9$ (2C), $128.0(2 \mathrm{C}), 127.5,126.9$, 109.2, 52.8, 46.7, 31.1, 29.7 (3C).

HRMS-DART $(\mathrm{m} / \mathrm{z}):[\mathrm{M}+\mathrm{H}]^{+}$calcd for $\mathrm{C}_{17} \mathrm{H}_{21} \mathrm{O}_{2}{ }^{+}, 257.1536$; found, 257.1536.

\section{6,6-Dimethyl-1,4-diphenylheptan-3-one (6aai)}

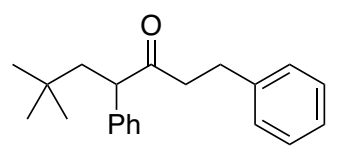

The product 6aai was purified by flash chromatography on silica gel (100:0-98:2, hexane/Et $\left.{ }_{2} \mathrm{O}\right)$ (Figure 3; $38.4 \mathrm{mg}, 0.129 \mathrm{mmol}, 64 \%$ isolated yield, containing 2\% impurity of two component coupling product). White solid.

M.p. $71-74{ }^{\circ} \mathrm{C}$.

IR (neat) 699, 747, 1030, 1090, 1365, 1453, 1475, 1494, 1714, 2865, $2953 \mathrm{~cm}^{-1}$. 
${ }^{1} \mathbf{H}$ NMR (400 MHz, $\left.\mathrm{CDCl}_{3}\right) \delta 7.29-7.26(\mathrm{~m}, 2 \mathrm{H}), 7.23-7.12(\mathrm{~m}, 6 \mathrm{H}), 7.05(\mathrm{~d}, J=7.2 \mathrm{~Hz}, 2 \mathrm{H})$, $3.71(\mathrm{dd}, J=7.6,4.0 \mathrm{~Hz}, 1 \mathrm{H}), 2.86-2.73(\mathrm{~m}, 4 \mathrm{H}), 2.34(\mathrm{dd}, J=14.0,7.6 \mathrm{~Hz}, 1 \mathrm{H}), 1.49$ (dd, $J=14.0$, $4.0 \mathrm{~Hz}, 1 \mathrm{H}), 0.81(\mathrm{~s}, 9 \mathrm{H})$.

${ }^{13} \mathrm{C} \mathrm{NMR}\left(100.6 \mathrm{MHz}, \mathrm{CDCl}_{3}\right) \delta 209.1,141.0,140.6,128.8(2 \mathrm{C}), 128.3(2 \mathrm{C}), 128.2(2 \mathrm{C} \times 2) 126.9$, 125.9, 55.7, 45.3, 43.3, 30.9, 29.9, 29.7 (3C).

HRMS-DART $(\mathrm{m} / \mathrm{z}):[\mathrm{M}+\mathrm{H}]^{+}$calcd for $\mathrm{C}_{21} \mathrm{H}_{27} \mathrm{O}^{+}, 295.2056$; found, 295.2054.

\section{8,8-Dimethyl-1,6-diphenylnonan-1,5-dione (6aaq)}

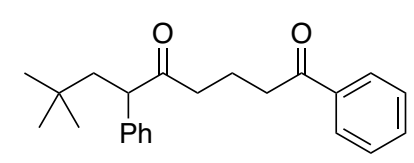

The product 6aaq was purified by flash chromatography on silica gel (100:0-90:10, hexane/Et $2 \mathrm{O})$ (Figure 3; $45.8 \mathrm{mg}, 0.134 \mathrm{mmol}, 67 \%$ isolated yield, containing 2\% impurity of two component coupling product). Pale yellow solid.

M.p. $62-66^{\circ} \mathrm{C}$.

IR (neat) 691, 701, 732, 753, 1225, 1365, 1449, 1684, 1712, $2952 \mathrm{~cm}^{-1}$.

${ }^{1} \mathbf{H}$ NMR $\left(400 \mathrm{MHz}, \mathrm{CDCl}_{3}\right) \delta 7.85(\mathrm{~d}, J=7.2 \mathrm{~Hz}, 2 \mathrm{H}), 7.53(\mathrm{t}, J=7.2 \mathrm{~Hz}, 1 \mathrm{H}), 7.42(\mathrm{dd}, J=7.2$, $7.2 \mathrm{~Hz}, 2 \mathrm{H}), 7.28-7.15(\mathrm{~m}, 5 \mathrm{H}), 3.76(\mathrm{~d}, J=7.6 \mathrm{~Hz}, 1 \mathrm{H}), 2.83(\mathrm{~m}, 1 \mathrm{H}), 2.74(\mathrm{~m}, 1 \mathrm{H}), 2.57(\mathrm{t}, J=6.8$ $\mathrm{Hz}, 2 \mathrm{H}), 2.36(\mathrm{dd}, J=14.0,7.6 \mathrm{~Hz}, 1 \mathrm{H}), 1.96-1.88(\mathrm{~m}, 2 \mathrm{H}), 1.50(\mathrm{~d}, J=14.0 \mathrm{~Hz}, 1 \mathrm{H}), 0.83(\mathrm{~s}, 9 \mathrm{H})$.

${ }^{13}$ C NMR (100.6 MHz, $\left.\mathrm{CDCl}_{3}\right) \delta 209.8,199.7,140.5,136.7,132.9,128.8$ (2C), 128.5 (2C), 128.2 (2C), 128.0 (2C), 127.0, 55.7, 45.3, 40.6, 37.2, 30.9, 29.7 (3C), 18.2.

HRMS-DART (m/z): [M+H $]^{+}$calcd for $\mathrm{C}_{23} \mathrm{H}_{29} \mathrm{O}_{2}{ }^{+}, 337.2162$; found, 337.2167.

\section{4,4-Dimethyl-1-(oxan-4-yl)-2-phenylpentan-1-one (6aar)}

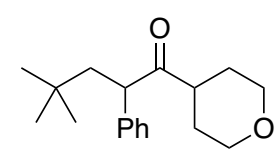

The product 6aar was purified by flash chromatography on silica gel (100:0-85:15, hexane/Et $2 \mathrm{O})$ (Figure 3; $37.9 \mathrm{mg}, 0.138 \mathrm{mmol}, 69 \%$ isolated yield). Pale yellow solid.

M.p. $77-81^{\circ} \mathrm{C}$.

IR (neat) 700, 723, 1023, 1093, 1115, 1240, 1365, 1708, 2846, $2951 \mathrm{~cm}^{-1}$.

${ }^{1} \mathbf{H}$ NMR $\left(400 \mathrm{MHz}, \mathrm{CDCl}_{3}\right) \delta 7.31-7.26(\mathrm{~m}, 2 \mathrm{H}), 7.24-7.19(\mathrm{~m}, 3 \mathrm{H}), 3.97(\mathrm{~d}, J=11.2 \mathrm{~Hz}, 1 \mathrm{H})$, $3.90(\mathrm{dd}, J=7.6,4.0 \mathrm{~Hz}, 1 \mathrm{H}), 3.85(\mathrm{~d}, J=11.2 \mathrm{~Hz}, 1 \mathrm{H}), 3.37(\mathrm{t}, J=11.6 \mathrm{~Hz}, 1 \mathrm{H}), 3.25(\mathrm{t}, J=11.6$ $\mathrm{Hz}, 1 \mathrm{H}), 2.63(\mathrm{~m}, 1 \mathrm{H}), 2.35(\mathrm{dd}, J=14.0,7.6 \mathrm{~Hz}, 1 \mathrm{H}), 1.77-1.58(\mathrm{~m}, 3 \mathrm{H}), 1.45(\mathrm{dd}, J=14.0,4.0 \mathrm{~Hz}$, 1H), $1.29(\mathrm{~d}, J=13.6 \mathrm{~Hz}, 1 \mathrm{H}), 0.84(\mathrm{~s}, 9 \mathrm{H})$.

${ }^{13} \mathrm{C}$ NMR (100.6 MHz, $\left.\mathrm{CDCl}_{3}\right) \delta 211.0,140.6,128.9$ (2C), 128.3 (2C), 127.0, 67.3, 67.1, 53.5, 47.1, 45.9, 30.9, 29.7 (3C), 29.1, 28.4.

HRMS-DART (m/z): $[\mathrm{M}+\mathrm{H}]^{+}$calcd for $\mathrm{C}_{18} \mathrm{H}_{27} \mathrm{O}_{2}{ }^{+}, 275.2006$; found, 275.2004. 


\section{1-Cyclohexyl-4,4-dimethyl-2-phenylpentan-1-one (6aas)}

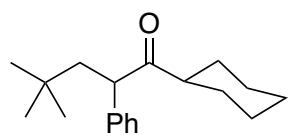

The product 6aas was purified by flash chromatography on silica gel (100:0-97.5:2.5, hexane/EtOAc) (Figure 3; $32.3 \mathrm{mg}, 0.117 \mathrm{mmol}, 58 \%$ isolated yield, containing 1\% impurity of two component coupling product). Pale yellow solid.

M.p. $55-56{ }^{\circ} \mathrm{C}$.

IR (neat) 699, 721, 989, 1365, 1451, 1474, 1492, 1708, 2855, $2931 \mathrm{~cm}^{-1}$.

${ }^{1} \mathbf{H}$ NMR $\left(400 \mathrm{MHz}, \mathrm{CDCl}_{3}\right) \delta 7.30-7.26(\mathrm{~m}, 2 \mathrm{H}), 7.22-7.19(\mathrm{~m}, 3 \mathrm{H}), 3.89$ (dd, $J=7.2,4.0 \mathrm{~Hz}$, $1 \mathrm{H}), 2.41(\mathrm{t}, J=10.8 \mathrm{~Hz}, 1 \mathrm{H}), 2.32(\mathrm{dd}, J=14.0,7.2 \mathrm{~Hz}, 1 \mathrm{H}), 1.87(\mathrm{~d}, J=10.8 \mathrm{~Hz}, 1 \mathrm{H}), 1.76(\mathrm{~d}, J=$ $10.8 \mathrm{~Hz}, 1 \mathrm{H}), 1.65-1.57(\mathrm{~m}, 2 \mathrm{H}), 1.45-1.06(\mathrm{~m}, 7 \mathrm{H}), 0.83(\mathrm{~s}, 9 \mathrm{H})$.

${ }^{13} \mathrm{C}$ NMR (100.6 MHz, $\left.\mathrm{CDCl}_{3}\right) \delta 212.9,141.0,128.7$ (2C), 128.4 (2C), 126.7, 53.8, 50.4, 46.0, 31.0, 29.7 (3C), 29.6, 28.7, 25.9, 25.7, 25.4.

HRMS-DART $(\mathrm{m} / \mathrm{z}):[\mathrm{M}+\mathrm{H}]^{+}$calcd for $\mathrm{C}_{19} \mathrm{H}_{29} \mathrm{O}^{+}, 273.2213$; found, 273.2218 .

\section{4,4-Dimethyl-1-(1-methylcyclohexyl)-2-phenylpentan-1-one (6aat)}

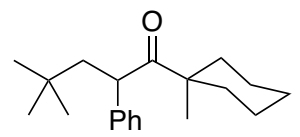

The product 6aat was purified by flash chromatography on silica gel (100:0-98:2, hexane/Et $2 \mathrm{O})$ (Figure 3; $24.4 \mathrm{mg}, 0.0852 \mathrm{mmol}, 43 \%$ isolated yield, containing inpurity). White solid.

M.p. $40-43{ }^{\circ} \mathrm{C}$.

IR (neat) 700, 730, 1012, 1364, 1456, 1493, 1558, 1699, 2864, $2933 \mathrm{~cm}^{-1}$.

${ }^{1} \mathbf{H}$ NMR $\left(400 \mathrm{MHz}, \mathrm{CDCl}_{3}\right) \delta 7.32(\mathrm{~d}, J=7.2 \mathrm{~Hz}, 2 \mathrm{H}), 7.25(\mathrm{dd}, J=7.2,7.2 \mathrm{~Hz}, 2 \mathrm{H}), 7.17(\mathrm{t}, J=$ $7.2 \mathrm{~Hz}, 1 \mathrm{H}), 4.28$ (dd, $J=8.4,4.4 \mathrm{~Hz}, 1 \mathrm{H}), 1.94-1.86(\mathrm{~m}, 2 \mathrm{H}), 1.82-1.74(\mathrm{~m}, 2 \mathrm{H}), 1.45-1.41$ (m, 2H), 1.34-1.21 (m, 6H), $1.05(\mathrm{~s}, 3 \mathrm{H}), 0.79(\mathrm{~s}, 9 \mathrm{H})$.

${ }^{13}$ C NMR (100.6 MHz, $\left.\mathrm{CDCl}_{3}\right) \delta 215.6,141.1,128.8$ (2C), 128.4 (2C), 126.5, 49.3, 49.0, 48.3, $34.8,34.5,31.3,30.0(3 \mathrm{C}), 25.8,23.8,22.5,22.4$.

HRMS-DART $(\mathrm{m} / \mathrm{z}):[\mathrm{M}+\mathrm{H}]^{+}$calcd for $\mathrm{C}_{20} \mathrm{H}_{31} \mathrm{O}^{+}, 287.2369$; found, 287.2380.

\section{6,6-Dimethyl-2-[4-[(2-oxocyclopentan-1-yl)methyl]phenyl]-4-phenylheptan-3-one (6aau)}<smiles>CC(C(=O)C(CC(C)(C)C)c1ccc(CC2CCCC2=O)cc1)c1ccccc1</smiles>

The product 6aau was purified by flash chromatography on silica gel (100:0-85:15, hexane/Et $\left.{ }_{2} \mathrm{O}\right)$ (Figure 3; $45.6 \mathrm{mg}, 0.117 \mathrm{mmol}, 58 \%$ isolated yield). Yellow oil. 
IR (neat) 700, 1154, 1366, 1453, 1474, 1492, 1511, 1713, 1739, 2868, $2956 \mathrm{~cm}^{-1}$.

Signals for two diastereomers (54:46) were given:

${ }^{1}$ H NMR $\left(400 \mathrm{MHz}, \mathrm{CDCl}_{3}\right) \delta 7.33-7.08(\mathrm{~m}, 6 \mathrm{H}), 6.93-6.91(\mathrm{~m}, 2 \mathrm{H}), 6.84(\mathrm{~m}, 1 \mathrm{H}), 3.89-3.70(\mathrm{~m}$, $2 \mathrm{H}), 3.08(\mathrm{~m}, 1 \mathrm{H}), 2.61-1.41(\mathrm{~m}, 10 \mathrm{H}), 1.37(\mathrm{~d}, J=6.8 \mathrm{~Hz}, 0.54 \times 3 \mathrm{H}), 1.22(\mathrm{~d}, J=6.8 \mathrm{~Hz}, 0.46 \times$ $3 \mathrm{H}), 0.81(\mathrm{~s}, 0.54 \times 9 \mathrm{H}), 0.53(\mathrm{~s}, 0.46 \times 9 \mathrm{H})$.

Signals for diastereomers were given:

${ }^{13}$ C NMR (100.6 MHz, $\left.\mathrm{CDCl}_{3}\right) \delta 220.1,210.7,209.3,141.25,141.22,140.7,139.0,138.9,138.6$, $138.5,138.34,138.32,137.75,137.72,129.5,129.4,129.0,128.8,128.5,128.28,128.26,128.1$, $126.9,126.4,126.3,54.1,54.0,53.2,52.61,52.58,50.99,50.94,50.89,50.87,47.00,46.95,45.2$, $38.3,38.23,38.21,38.18,35.11,35.07,35.04,31.1,30.9,30.8,29.7,29.3,29.10,29.06,28.9,28.8$, $25.0,20.5,18.12,18.08,17.51,17.46$ (only observed peaks).

HRMS-DART $(\mathrm{m} / \mathrm{z}):[\mathrm{M}+\mathrm{H}]^{+}$calcd for $\mathrm{C}_{27} \mathrm{H}_{35} \mathrm{O}_{2}{ }^{+}, 391.2632$; found, 391.2628.

\section{4,4,8,8-Tetramethyl-1-(2,5-dimethylphenoxy)-6-phenylnonan-5-one (6aav)}

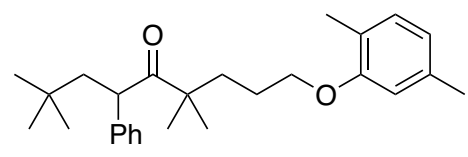

The product 6aav was purified by flash chromatography on silica gel (95:5, hexane/Et $2 \mathrm{O})$ (Figure 3; $26.0 \mathrm{mg}, 0.0659 \mathrm{mmol}, 33 \%$ isolated yield). Colourless oil.

IR (neat) 700, 732, 1028, 1130, 1157, 1265, 1365, 1472, 1509, 1699, $2954 \mathrm{~cm}^{-1}$.

${ }^{1} \mathbf{H}$ NMR $\left(400 \mathrm{MHz}, \mathrm{CDCl}_{3}\right) \delta 7.31(\mathrm{~d}, J=7.2 \mathrm{~Hz}, 2 \mathrm{H}), 7.25(\mathrm{dd}, J=7.2,7.2 \mathrm{~Hz}, 2 \mathrm{H}), 7.17(\mathrm{t}, J=$ $7.2 \mathrm{~Hz}, 1 \mathrm{H}), 6.99$ (d, $J=7.2 \mathrm{~Hz}, 1 \mathrm{H}), 6.65(\mathrm{~d}, J=7.2 \mathrm{~Hz}, 1 \mathrm{H}), 6.54(\mathrm{~s}, 1 \mathrm{H}), 4.27$ (dd, $J=7.2,4.8 \mathrm{~Hz}$, 1H), 3.77-3.69 (m, 2H), 2.30 (s, 3H), 2.14 (s, 3H), 1.99 (dd, J=14.4, 4.8 Hz, 1H), 1.71 (dd, $J=14.4$, $7.2 \mathrm{~Hz}, 1 \mathrm{H}), 1.65-1.52(\mathrm{~m}, 3 \mathrm{H}), 1.33(\mathrm{~m}, 1 \mathrm{H}), 1.13(\mathrm{~s}, 6 \mathrm{H}), 0.80(\mathrm{~s}, 9 \mathrm{H})$.

${ }^{13}$ C NMR $\left(100.6 \mathrm{MHz}, \mathrm{CDCl}_{3}\right) \delta 214.9,156.9,140.8,136.4,130.2,128.7$ (2C), 128.5 (2C), 126.6, 123.6, 120.6, 112.0, 68.0, 49.3, 48.6, 48.2, 36.5, 31.3, 29.9 (3C), 25.2, 24.74, 24.69, 21.4, 15.8.

HRMS-DART (m/z): [M+H] $]^{+}$calcd for $\mathrm{C}_{27} \mathrm{H}_{39} \mathrm{O}_{2}{ }^{+}, 395.2945$; found, 395.2940.

\section{2-(4-Methoxyphenyl)-4,4-dimethyl-1-(4-methylphenyl)pentan-1-one (6aba)}

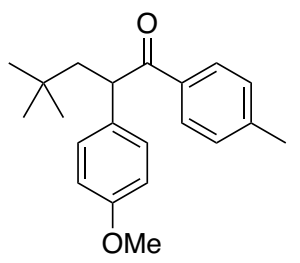

The product 6aba was purified by flash chromatography on silica gel (100:0-95:5, hexane/Et $2 \mathrm{O})$ (Figure 3; $39.3 \mathrm{mg}, 0.126 \mathrm{mmol}, 63 \%$ isolated yield, containing trace inpurity of two component coupling product). Pale yellow solid.

M.p. $78-82{ }^{\circ} \mathrm{C}$.

IR (neat) 1177, 1251, 1457, 1473, 1508, 1541, 1558, 1607, 1653, 1683, $2953 \mathrm{~cm}^{-1}$. 
${ }^{1} \mathbf{H}$ NMR $\left(400 \mathrm{MHz}, \mathrm{CDCl}_{3}\right) \delta 7.90(\mathrm{~d}, J=7.6 \mathrm{~Hz}, 2 \mathrm{H}), 7.23-7.20(\mathrm{~m}, 4 \mathrm{H}), 6.79(\mathrm{~d}, J=7.6 \mathrm{~Hz}$, $2 \mathrm{H}), 4.65(\mathrm{~d}, J=9.2 \mathrm{~Hz}, 1 \mathrm{H}), 3.74(\mathrm{~s}, 3 \mathrm{H}), 2.57(\mathrm{dd}, J=14.0,9.2 \mathrm{~Hz}, 1 \mathrm{H}), 2.36(\mathrm{~s}, 3 \mathrm{H}), 1.54(\mathrm{~d}, J=$ $14.0 \mathrm{~Hz}, 1 \mathrm{H}), 0.87$ (s, 9H).

${ }^{13}$ C NMR (100.6 MHz, $\left.\mathrm{CDCl}_{3}\right) \delta 199.8,158.3,143.5,134.5,133.3,129.2$ (2C), 129.1 (2C), 128.7 (2C), 114.2 (2C), 55.2, 48.4, 47.5, 31.1, $29.8(3 \mathrm{C}), 21.6$.

HRMS-DART $(\mathrm{m} / \mathrm{z})$ : $[\mathrm{M}+\mathrm{H}]^{+}$calcd for $\mathrm{C}_{21} \mathrm{H}_{27} \mathrm{O}_{2}{ }^{+}, 311.2006$; found, 311.2003.

\section{2-(4-Chlorophenyl)-4,4-dimethyl-1-(4-methylphenyl)pentan-1-one (6aca)}

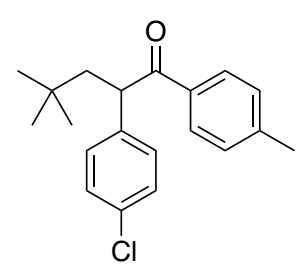

The product 6aca was purified by flash chromatography on silica gel (100:0-95:5, hexane/Et $2 \mathrm{O})$ (Figure 3; $31.5 \mathrm{mg}, 0.0993 \mathrm{mmol}, 50 \%$ isolated yield, containing trace impurity of two component coupling product). Pale yellow solid.

M.p. $96-97^{\circ} \mathrm{C}$.

IR (neat) 753, 828, 1015, 1092, 1175, 1365, 1489, 1607, 1681, $2955 \mathrm{~cm}^{-1}$.

${ }^{1} \mathbf{H}$ NMR $\left(400 \mathrm{MHz}, \mathrm{CDCl}_{3}\right) \delta 7.88(\mathrm{~d}, J=7.6 \mathrm{~Hz}, 2 \mathrm{H}), 7.26-7.21(\mathrm{~m}, 6 \mathrm{H}), 4.68(\mathrm{~d}, J=8.8 \mathrm{~Hz}$, $1 \mathrm{H}), 2.57(\mathrm{dd}, J=14.0,8.8 \mathrm{~Hz}, 1 \mathrm{H}), 2.37(\mathrm{~s}, 3 \mathrm{H}), 1.54(\mathrm{~d}, J=14.0 \mathrm{~Hz}, 1 \mathrm{H}), 0.87(\mathrm{~s}, 9 \mathrm{H})$.

${ }^{13}$ C NMR (100.6 MHz, $\left.\mathrm{CDCl}_{3}\right) \delta 199.3,143.8,139.8,134.2,132.5,129.5$ (2C), 129.4 (2C), 128.9 (2C), 128.7 (2C), 48.7, 47.5, 31.2, 29.8 (3C), 21.6.

HRMS-DART $(\mathrm{m} / \mathrm{z})$ : $[\mathrm{M}+\mathrm{H}]^{+}$calcd for $\mathrm{C}_{20} \mathrm{H}_{24} \mathrm{ClO}^{+}, 315.1510$; found, 315.1508 .

\section{2-(4-Bromophenyl)-4,4-dimethyl-1-(4-methylphenyl)pentan-1-one (6ada)}

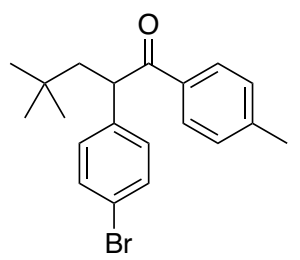

The product 6ada was purified by flash chromatography on silica gel (100:0-97.5:2.5, hexane/Et ${ }_{2} \mathrm{O}$ ) (Figure 3; $45.7 \mathrm{mg}, 0.126 \mathrm{mmol}, 63 \%$ isolated yield, containing $1 \%$ impurity of two component coupling product). White solid.

M.p. $91-93{ }^{\circ} \mathrm{C}$.

IR (neat) $1011,1175,1487,1507,1540,1558,1607,1653,1683,2955 \mathrm{~cm}^{-1}$.

${ }^{1} \mathbf{H}$ NMR $\left(400 \mathrm{MHz}, \mathrm{CDCl}_{3}\right) \delta 7.88(\mathrm{~d}, J=8.0 \mathrm{~Hz}, 2 \mathrm{H}), 7.38(\mathrm{~d}, J=8.0 \mathrm{~Hz}, 2 \mathrm{H}), 7.23-7.18(\mathrm{~m}$, $4 \mathrm{H}), 4.67(\mathrm{~d}, J=8.8 \mathrm{~Hz}, 1 \mathrm{H}), 2.57(\mathrm{dd}, J=14.0,8.8 \mathrm{~Hz}, 1 \mathrm{H}), 2.37(\mathrm{~s}, 3 \mathrm{H}), 1.54(\mathrm{~d}, J=14.0 \mathrm{~Hz}, 1 \mathrm{H})$, $0.87(\mathrm{~s}, 9 \mathrm{H})$. 
${ }^{13}$ C NMR (100.6 MHz, $\left.\mathrm{CDCl}_{3}\right) \delta 199.2,143.9,140.3,134.1,131.9$ (2C), 129.8 (2C), $129.4(2 \mathrm{C})$, 128.7 (2C), 120.6, 48.7, 47.4, 31.2, 29.8 (3C), 21.6.

HRMS-DART $(\mathrm{m} / \mathrm{z})$ : $[\mathrm{M}+\mathrm{H}]^{+}$calcd for $\mathrm{C}_{20} \mathrm{H}_{24} \mathrm{BrO}^{+}, 359.1005$; found, 359.1009 .

\section{2-[4-(Methoxycarbonyl)phenyl]-4,4-dimethyl-1-(4-methylphenyl)pentan-1-one (6aea)}

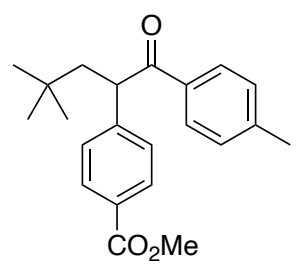

The product 6aea was purified by flash chromatography on silica gel (100:0-85:15, hexane/Et $2 \mathrm{O})$ (Figure 3; $45.6 \mathrm{mg}, 0.135 \mathrm{mmol}, 67 \%$ isolated yield). White solid.

M.p. $103-105^{\circ} \mathrm{C}$.

IR (neat) 741,1112, 1180, 1250, 1280, 1435, 1607, 1682, 1722, $2952 \mathrm{~cm}^{-1}$.

${ }^{1} \mathbf{H}$ NMR $\left(400 \mathrm{MHz}, \mathrm{CDCl}_{3}\right) \delta 7.93(\mathrm{~d}, J=8.0 \mathrm{~Hz}, 2 \mathrm{H}), 7.89(\mathrm{~d}, J=8.0 \mathrm{~Hz}, 2 \mathrm{H}), 7.39(\mathrm{~d}, J=8.0$ $\mathrm{Hz}, 2 \mathrm{H}), 7.21(\mathrm{~d}, J=8.0 \mathrm{~Hz}, 2 \mathrm{H}), 4.76(\mathrm{~d}, J=8.4 \mathrm{~Hz}, 1 \mathrm{H}), 3.87(\mathrm{~s}, 3 \mathrm{H}), 2.61$ (dd, $J=14.0,8.4 \mathrm{~Hz}$, $1 \mathrm{H}), 2.37(\mathrm{~s}, 3 \mathrm{H}), 1.57$ (d, $J=14.0 \mathrm{~Hz}, 1 \mathrm{H}), 0.88(\mathrm{~s}, 9 \mathrm{H})$.

${ }^{13}$ C NMR (100.6 MHz, $\left.\mathrm{CDCl}_{3}\right) \delta 198.9,166.8,146.5,143.9,134.2,130.1$ (2C), 129.4 (2C), 128.7 (2C), 128.6, $128.2(2 \mathrm{C}), 52.0,49.5,47.4,31.3,29.8$ (3C), 21.6.

HRMS-DART (m/z): [M+H $]^{+}$calcd for $\mathrm{C}_{22} \mathrm{H}_{27} \mathrm{O}_{3}{ }^{+}, 339.1955$; found, 339.1965.

\section{2-(4-(Trifluoromethyl)phenyl)-4,4-dimethyl-1-(4-methylphenyl)pentan-1-one (6afa)}

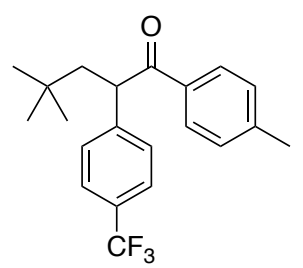

The product 6afa was purified by flash chromatography on silica gel (100:0-98:2, hexane/Et $\left.\mathrm{E}_{2} \mathrm{O}\right)$ (Figure 3; $36.0 \mathrm{mg}, 0.103 \mathrm{mmol}, 52 \%$ isolated yield, containing trace amount of impurity of two component coupling product). White solid.

M.p. $80-83^{\circ} \mathrm{C}$.

IR (neat) 1019, 1069, 1110, 1126, 1165, 1325, 1607, 1615, 1681, $2958 \mathrm{~cm}^{-1}$.

${ }^{1} \mathbf{H}$ NMR $\left(400 \mathrm{MHz}, \mathrm{CDCl}_{3}\right) \delta 7.89(\mathrm{~d}, J=7.6 \mathrm{~Hz}, 2 \mathrm{H}), 7.52(\mathrm{~d}, J=8.0 \mathrm{~Hz}, 2 \mathrm{H}), 7.44(\mathrm{~d}, J=8.0$ $\mathrm{Hz}, 2 \mathrm{H}), 7.23(\mathrm{~d}, J=7.6 \mathrm{~Hz}, 2 \mathrm{H}), 4.78(\mathrm{~d}, J=9.2 \mathrm{~Hz}, 1 \mathrm{H}), 2.62(\mathrm{dd}, J=13.6,9.2 \mathrm{~Hz}, 1 \mathrm{H}), 2.38(\mathrm{~s}$, $3 \mathrm{H}), 1.56(\mathrm{~d}, J=13.6 \mathrm{~Hz}, 1 \mathrm{H}), 0.89(\mathrm{~s}, 9 \mathrm{H})$.

${ }^{13} \mathrm{C}$ NMR $\left(100.6 \mathrm{MHz}, \mathrm{CDCl}_{3}\right) \delta 198.9,145.3,144.1,134.0,129.4(2 \mathrm{C}), 129.0\left(\mathrm{q}, J_{\mathrm{C}-\mathrm{F}}=32.3 \mathrm{~Hz}\right.$, $1 \mathrm{C}), 128.7(2 \mathrm{C}), 128.4(2 \mathrm{C}), 125.7\left(\mathrm{q}, J_{\mathrm{C}-\mathrm{F}}=3.5 \mathrm{~Hz}, 2 \mathrm{C}\right), 124.1\left(\mathrm{q}, J_{\mathrm{C}-\mathrm{F}}=272.0 \mathrm{~Hz}, 1 \mathrm{C}\right), 49.1,47.5$, $31.3,29.8(3 \mathrm{C}), 21.6$.

${ }^{19}$ F NMR $\left(376.5 \mathrm{MHz}, \mathrm{CDCl}_{3}\right) \delta-62.5$. 
HRMS-DART $(\mathrm{m} / \mathrm{z})$ : $[\mathrm{M}+\mathrm{H}]^{+}$calcd for $\mathrm{C}_{21} \mathrm{H}_{24} \mathrm{~F}_{3} \mathrm{O}^{+}, 349.1774$; found, 349.1768 .

\section{4,4-Dimethyl-1-(4-methylphenyl)-2-(2-naphthyl)pentan-1-one (6aga)}

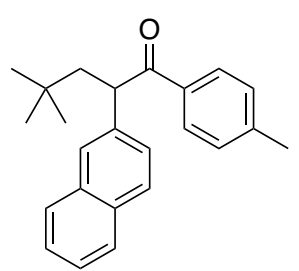

The product 6aga was purified by flash chromatography on silica gel (100:0-97:3, hexane/Et $\left.{ }_{2} \mathrm{O}\right)$ (Figure 3; $39.5 \mathrm{mg}, 0.120 \mathrm{mmol}, 60 \%$ isolated yield). Pale pink solid.

M.p. $118-121^{\circ} \mathrm{C}$.

IR (neat) 755, 818, 975, 1181, 1222, 1282, 1365, 1475, 1606, 1678, $2954 \mathrm{~cm}^{-1}$.

${ }^{1} \mathbf{H}$ NMR $\left(400 \mathrm{MHz}, \mathrm{CDCl}_{3}\right) \delta 7.94(\mathrm{~d}, J=7.6 \mathrm{~Hz}, 2 \mathrm{H}), 7.77-7.75(\mathrm{~m}, 3 \mathrm{H}), 7.72(\mathrm{~s}, 1 \mathrm{H}), 7.47(\mathrm{~d}$, $J=8.4 \mathrm{~Hz}, 1 \mathrm{H}), 7.44-7.38(\mathrm{~m}, 2 \mathrm{H}), 7.19(\mathrm{~d}, J=7.6 \mathrm{~Hz}, 2 \mathrm{H}), 4.86(\mathrm{~d}, J=8.8 \mathrm{~Hz}, 1 \mathrm{H}), 2.71(\mathrm{dd}, J=$ 14.0, $8.8 \mathrm{~Hz}, 1 \mathrm{H}), 2.34$ (s, 3H), 1.64 (d, $J=14.0 \mathrm{~Hz}, 1 \mathrm{H}), 0.91(\mathrm{~s}, 9 \mathrm{H})$.

${ }^{13} \mathrm{C}$ NMR $\left(100.6 \mathrm{MHz}, \mathrm{CDCl}_{3}\right) \delta 199.5,143.6,138.8,134.5,133.6,132.3,129.3$ (2C), $128.8(2 \mathrm{C})$, $128.6,127.7,127.6,126.7,126.3,126.1,125.6,49.6,47.5,31.3,29.8(3 \mathrm{C}), 21.5$.

HRMS-DART (m/z): $[\mathrm{M}+\mathrm{H}]^{+}$calcd for $\mathrm{C}_{24} \mathrm{H}_{27} \mathrm{O}^{+}, 331.2056$; found, 331.2053.

\section{4,4-Dimethyl-1-(4-methylphenyl)-2-(thiophen-2-yl)pentan-1-one (6aha)}

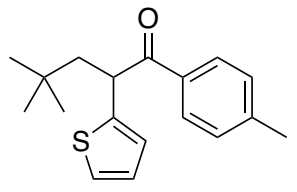

The product 6aha was purified by flash chromatography on silica gel (100:0-97:3, hexane/Et $\left.{ }_{2} \mathrm{O}\right)$ (Figure 3; $28.1 \mathrm{mg}, 0.0977 \mathrm{mmol}, 49 \%$ isolated yield, containing trace amount of impurity of two component coupling product). Pale yellow solid.

M.p. $65-67^{\circ} \mathrm{C}$.

IR (neat) 695, 1181, 1228, 1280, 1365, 1475, 1606, 1682, 2865, $2954 \mathrm{~cm}^{-1}$.

${ }^{1} \mathbf{H}$ NMR $\left(400 \mathrm{MHz}, \mathrm{CDCl}_{3}\right) \delta 7.94(\mathrm{~d}, J=7.6 \mathrm{~Hz}, 2 \mathrm{H}), 7.25(\mathrm{~d}, J=7.6 \mathrm{~Hz}, 2 \mathrm{H}), 7.13(\mathrm{~m}, 1 \mathrm{H})$, $6.86(\mathrm{~s}, 2 \mathrm{H}), 5.00(\mathrm{~d}, J=8.8 \mathrm{~Hz}, 1 \mathrm{H}), 2.58(\mathrm{dd}, J=14.0,8.8 \mathrm{~Hz}, 1 \mathrm{H}), 2.39$ (s, 3H), $1.72(\mathrm{~d}, J=14.0$ $\mathrm{Hz}, 1 \mathrm{H}), 0.89$ (s, 9H).

${ }^{13}$ C NMR (100.6 MHz, $\left.\mathrm{CDCl}_{3}\right) \delta 198.4,143.92,143.91,133.8,129.4$ (2C), 128.8 (2C), 126.8, 125.0, 124.4, 48.2, 43.9, 31.1, 29.7 (3C), 21.6.

HRMS-DART $(\mathrm{m} / \mathrm{z}):[\mathrm{M}+\mathrm{H}]^{+}$calcd for $\mathrm{C}_{18} \mathrm{H}_{23} \mathrm{OS}^{+}, 287.1464$; found, 287.1471.

\section{Methyl 4,4-dimethyl-2-(4-methylbenzoyl)pentanoate (6aia)}




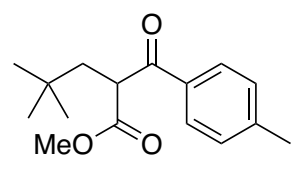

The product 6aia was purified by flash chromatography on silica gel (85:15, hexane/Et $2 \mathrm{O})$ (Figure 3; $6.2 \mathrm{mg}, 0.024 \mathrm{mmol}, 12 \%$ isolated yield, containing unidentified impurity). Colourless oil.

IR (neat) 1077, 1155, 1182, 1230, 1284, 1367, 1608, 1683, 1740, $2953 \mathrm{~cm}^{-1}$.

${ }^{1} \mathbf{H}$ NMR $\left(400 \mathrm{MHz}, \mathrm{CDCl}_{3}\right) \delta 7.91(\mathrm{~d}, J=8.0 \mathrm{~Hz}, 2 \mathrm{H}), 7.28(\mathrm{~d}, J=8.0 \mathrm{~Hz}, 2 \mathrm{H}), 4.40$ (t, $J=6.0$ $\mathrm{Hz}, 1 \mathrm{H}), 3.67$ (s, 3H), 2.42 (s, 3H), 2.03 (d, $J=6.0 \mathrm{~Hz}, 2 \mathrm{H}), 0.90$ (s, 9H).

${ }^{13} \mathrm{C}$ NMR (100.6 MHz, $\left.\mathrm{CDCl}_{3}\right) \delta 195.0,171.1,144.4,133.5,129.5$ (2C), 128.9 (2C), 52.5, 50.5, 42.0, 30.9, 29.3 (3C), 21.7.

HRMS-DART $(\mathrm{m} / \mathrm{z}):[\mathrm{M}+\mathrm{H}]^{+}$calcd for $\mathrm{C}_{16} \mathrm{H}_{23} \mathrm{O}_{3}{ }^{+}, 263.1642$; found, 263.1641 . 


\section{References}

(1) Sumida, Y.; Harada, R.; Kato-Sumida, T.; Johmoto, K.; Uekusa, H.; Hosoya, T., Boron-Selective Biaryl Coupling Approach to Versatile Dibenzoxaborins and Application to Concise Synthesis of Defucogilvocarcin M. Org. Lett. 2014, 16, 6240-6243.

(2) Numano, M.; Nagami, N.; Nakatsuka, S.; Katayama, T.; Nakajima, K.; Tatsumi, S.; Yasuda, N.; Hatakeyama, T., Synthesis of Boronate-Based Benzo[fg]tetracene and Benzo[hi]hexacene via Demethylative Direct Borylation. Chem. Eur. J. 2016, 22, 11574-7.

(3) Ling, K. B.; Smith, A. D., $\alpha$-Aroyloxyaldehydes: scope and limitations as alternatives to $\alpha$ haloaldehydes for NHC-catalysed redox transformations. Chem. Commun. 2011, 47, 373-375.

(4) Kyan, R.; Sato, K.; Mase, N.; Narumi, T., Pendant Alkoxy Groups on N-Aryl Substitutions Drive the Efficiency of Imidazolylidene Catalysts for Homoenolate Annulation from Enal and Aldehyde. Angew. Chem. Int. Ed. 2020, 59, 19031-19036.

(5) Draskovits, M.; Kalaus, H.; Stanetty, C.; Mihovilovic, M. D., Intercepted dehomologation of aldoses by N-heterocyclic carbene catalysis - a novel transformation in carbohydrate chemistry. Chem. Commun. 2019, 55, 12144-12147.

(6) Mukherjee, J., Use of Diethylaminosulfur Trifluoride in an Efficient Synthesis of (S)-N-[(1-Ethyl2-Pyrrolidinyl)Methyl]-5-(3-Fluoropropyl-2-Methoxybenzamide. J. Fluorine Chem. 1990, 49, 151154.

(7) Babadzhanova, L. A.; Kirij, N. V.; Yagupolskii, Y. L.; Tyrra, W.; Naumann, D., Convenient syntheses of 1,1,1,3,3,3-hexafluoro-2-organyl-propan-2-ols and the corresponding trimethylsilyl ethers. Tetrahedron 2005, 61, 1813-1819.

(8) Gockel, S. N.; Lee, S.; Gay, B. L.; Hull, K. L., Oxidative Three-Component Carboamination of Vinylarenes with Alkylboronic Acids. ACS Catal. 2021, 11, 5166-5171.

(9) Sato, Y.; Nakamura, K.; Sumida, Y.; Hashizume, D.; Hosoya, T.; Ohmiya, H., Generation of Alkyl Radical through Direct Excitation of Boracene-Based Alkylborate. J. Am. Chem. Soc. 2020, 142, 9938-9943.

(10) Miyamoto, Y.; Sumida, Y.; Ohmiya, H., Generation of Functionalized Alkyl Radicals via the Direct Photoexcitation of 2,2'-(Pyridine-2,6-diyl)diphenol-Based Borates. Org. Lett. 2021, 23, 58655870 .

(11) Davies, A. V.; Fitzpatrick, K. P.; Betori, R. C.; Scheidt, K. A., Combined Photoredox and Carbene Catalysis for the Synthesis of Ketones from Carboxylic Acids. Angew. Chem. Int. Ed. 2020, 59, 9143-9148.

(12) Ishii, T.; Kakeno, Y.; Nagao, K.; Ohmiya, H., N-Heterocyclic Carbene-Catalyzed Decarboxylative Alkylation of Aldehydes. J. Am. Chem. Soc. 2019, 141, 3854-3858.

(13) Peyman, A.; Hickl, E.; Beckhaus, H.-D., Anwendung von Kraftfeldrechnungen, 7 Synthese und Thermolyse 1,1'-disubstituierter trans-Azoneopentane. - Resonanzeffekt und sterischer Einfluß der Substituenten auf die thermische Stabilität sekundärer Azoalkane. Chem. Ber. 1987, 120, 713-725.

(14) Wang, L.; Wang, T.; Cheng, G.-J.; Li, X.; Wei, J.-J.; Guo, B.; Zheng, C.; Chen, G.; Ran, C.; Zheng, C., Direct C-H Arylation of Aldehydes by Merging Photocatalyzed Hydrogen Atom Transfer with Palladium Catalysis. ACS Catal. 2020, 10, 7543-7551. 
(15) Mamidala, R.; Biswal, P.; Subramani, M. S.; Samser, S.; Venkatasubbaiah, K., PalladacyclePhosphine Catalyzed Methylation of Amines and Ketones Using Methanol. J. Org. Chem. 2019, 84, 10472-10480.

(16) Tao, L.; Guo, X.; Li, J.; Li, R.; Lin, Z.; Zhao, W., Rhodium-Catalyzed Deoxygenation and Borylation of Ketones: A Combined Experimental and Theoretical Investigation. J. Am. Chem. Soc. 2020, 142, 18118-18127.

(17) Qi, L.; Li, R.; Yao, X.; Zhen, Q.; Ye, P.; Shao, Y.; Chen, J., Syntheses of Pyrroles, Pyridines, and Ketonitriles via Catalytic Carbopalladation of Dinitriles. J. Org. Chem. 2020, 85, 1097-1108.

(18) Fairlamb, I. J. S.; Kapdi, A. R.; Lee, A. F., ๆ2-dba Complexes of Pd(0): The Substituent Effect in Suzuki-Miyaura Coupling. Org. Lett. 2004, 6, 4435-4438.

(19) Zhan, M.; Zhang, T.; Huang, H.; Xie, Y.; Chen, Y., A simple method for $\alpha$-position deuterated carbonyl compounds with pyrrolidine as catalyst. J. Labelled Compd. Radiopharm. 2014, 57, 533539.

(20) Joshua, C. P.; Li, Ramadas, P. K., Photolysis of 2,2'-Dinitrodiphenylmethanes. A New Route to the Dibenzo[ $c, f][1,2]$ diazepine System Synthesis 2011, 6, 873-880.

(21) Ogiwara, Y.; Miyake, M.; Kochi, T.; Kakiuchi, F., Syntheses of RuHCl(CO)(PAr3)3 and $\mathrm{RuH} 2(\mathrm{CO})(\mathrm{PAr} 3) 3$ Containing Various Triarylphosphines and Their Use for Arylation of Sterically Congested Aromatic C-H Bonds. Organometallics 2017, 36, 159-164.

(22) Takuwa, T.; Minowa, T.; Fujisawa, H.; Mukaiyama, T., A Facile One-Pot Benzylation of Sodium Enolates Using Trifluoromethanesulfonic Anhydride and Diphenyl Sulfoxide. Chem. Pharm. Bull. 2005, 53, 476-480.

(23) Li, B. X.; Le, D. N.; Mack, K. A.; McClory, A.; Lim, N.-K.; Cravillion, T.; Savage, S.; Han, C.; Collum, D. B.; Zhang, H.; Gosselin, F., Highly Stereoselective Synthesis of Tetrasubstituted Acyclic All-Carbon Olefins via Enol Tosylation and Suzuki-Miyaura Coupling. J. Am. Chem. Soc. 2017, 139, 10777-10783.

(24) Pan, C.; Ni, Q.; Fu, Y.; Yu, J.-T., Radical 1,2-Alkylarylation/Acylarylation of Allylic Alcohols with Aldehydes via Neophyl Rearrangement. J. Org. Chem. 2017, 82, 7683-7688. 

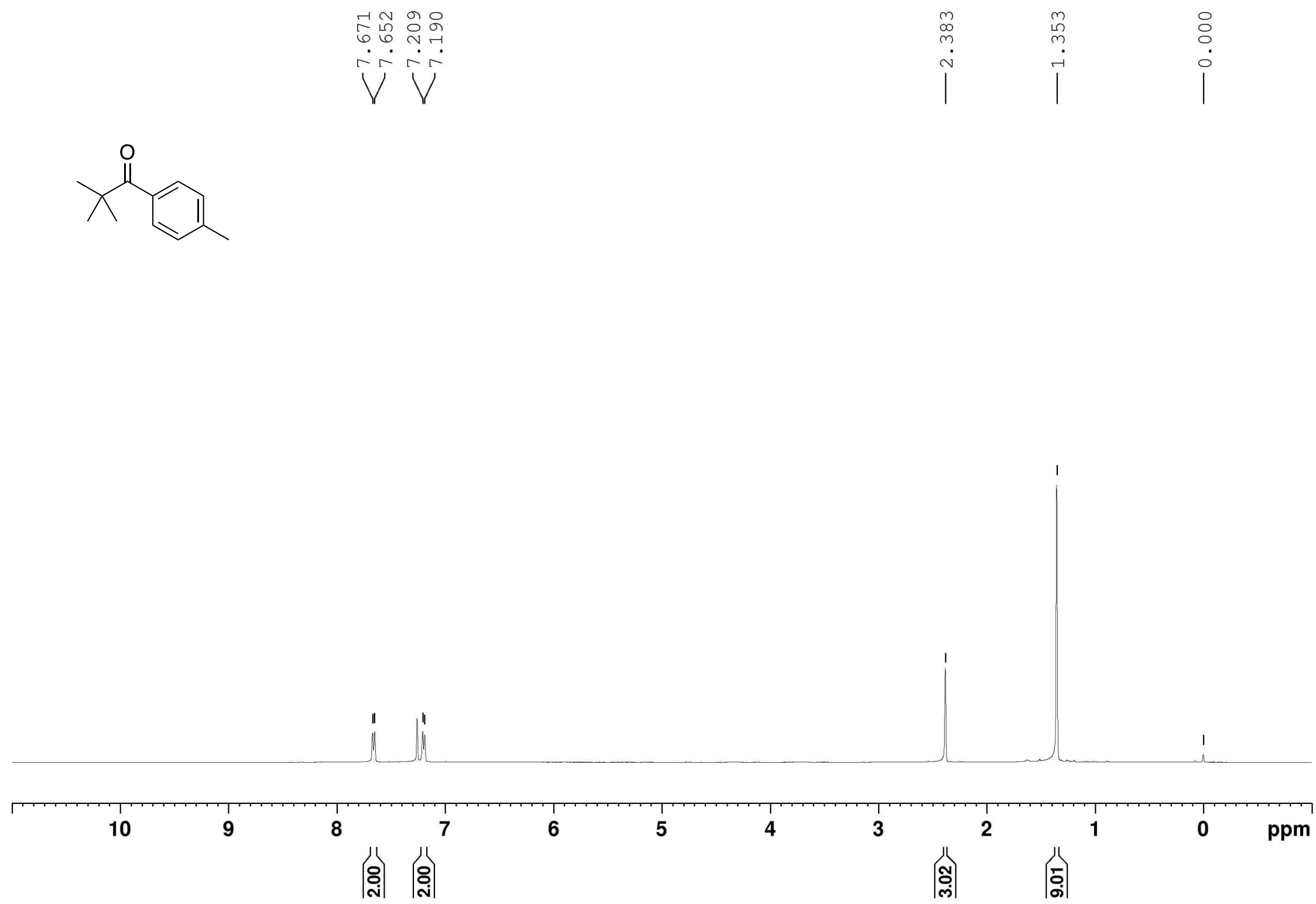
${ }^{13} \mathrm{C}$ NMR of 4 aa

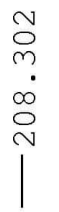
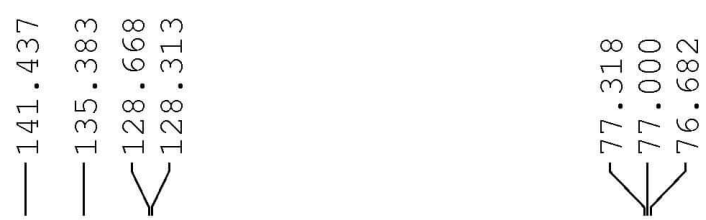

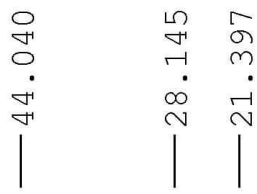
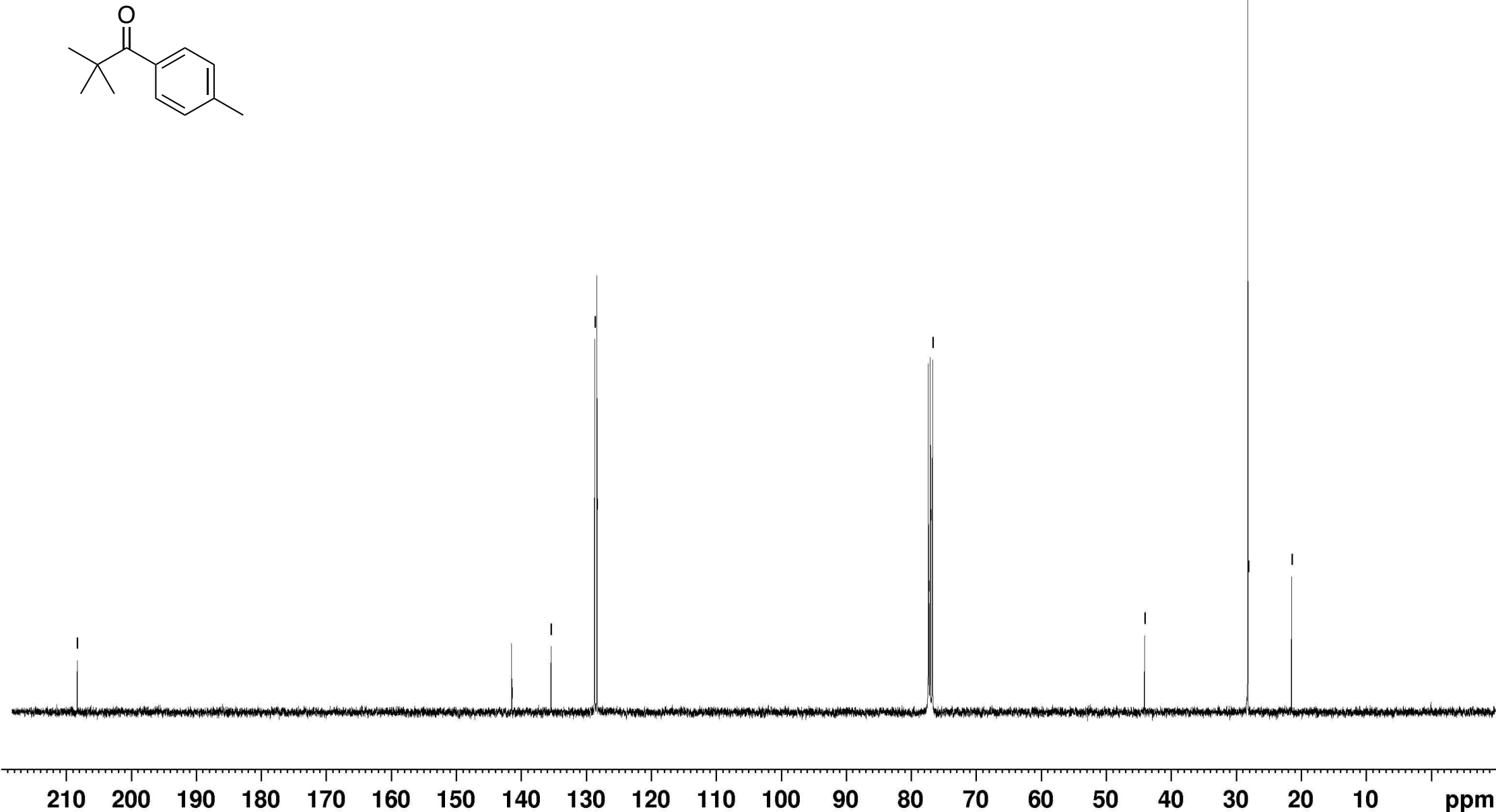
${ }^{1} \mathrm{H}$ NMR of $\mathbf{4 a b}$
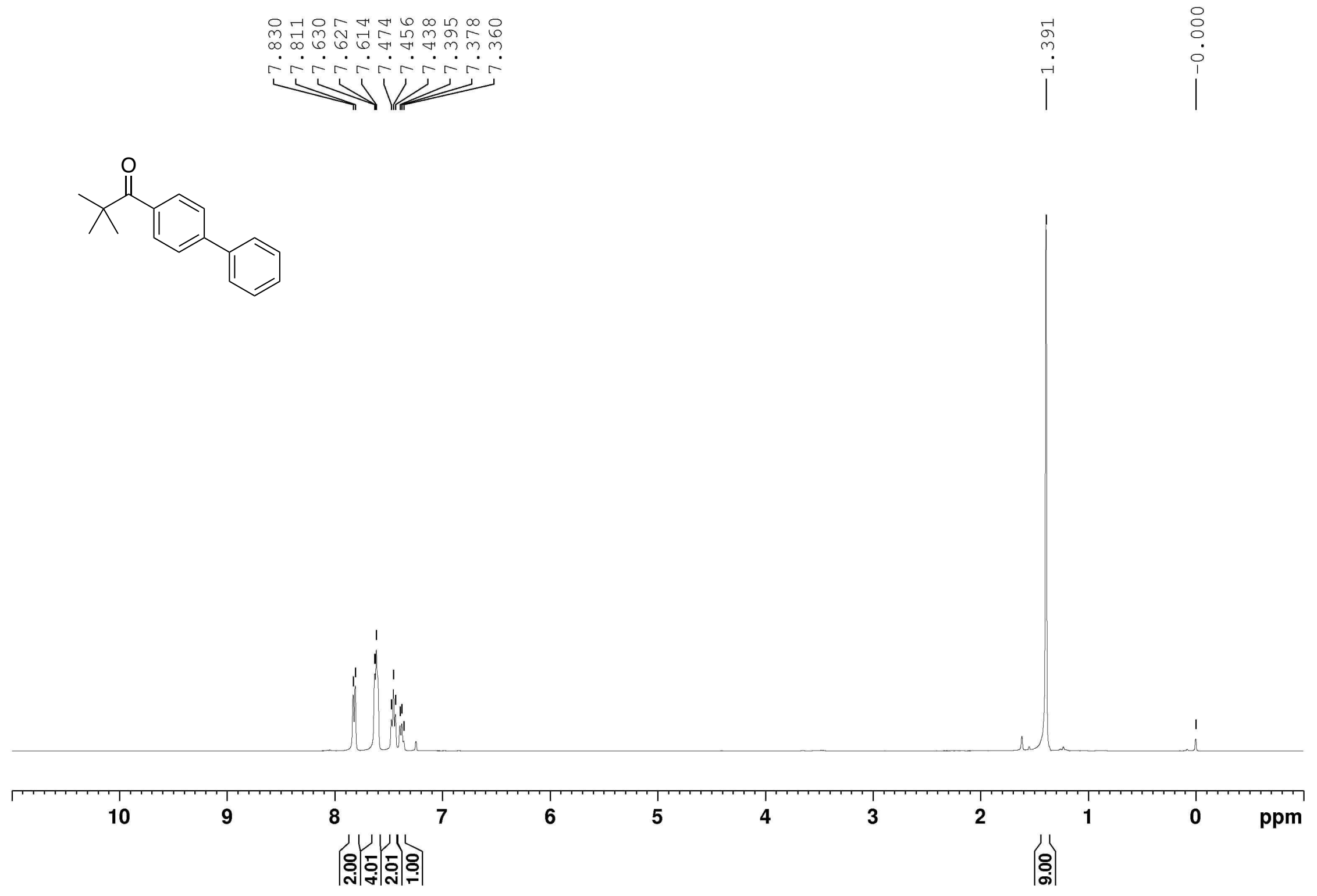

S50 
${ }^{13} \mathrm{C}$ NMR of $\mathbf{4 a b}$
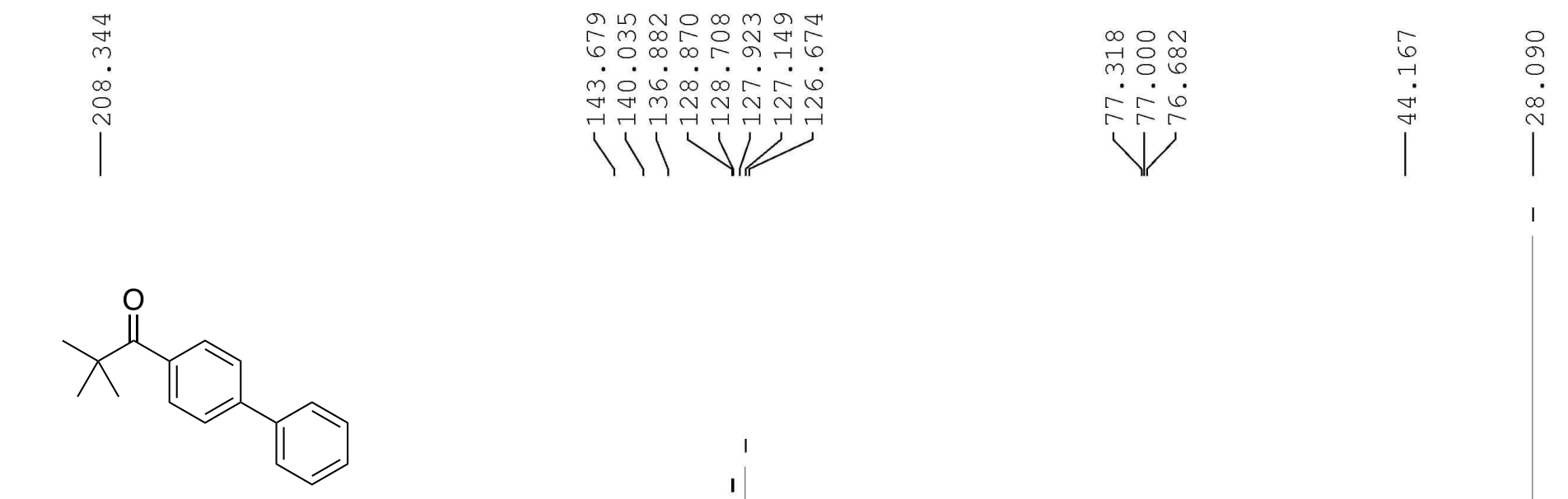

$\stackrel{+}{\not}$
$\stackrel{1}{\infty}$
$\infty$
$\stackrel{0}{\circ}$
$\mid$

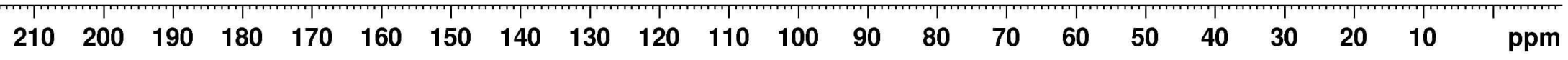

S51 


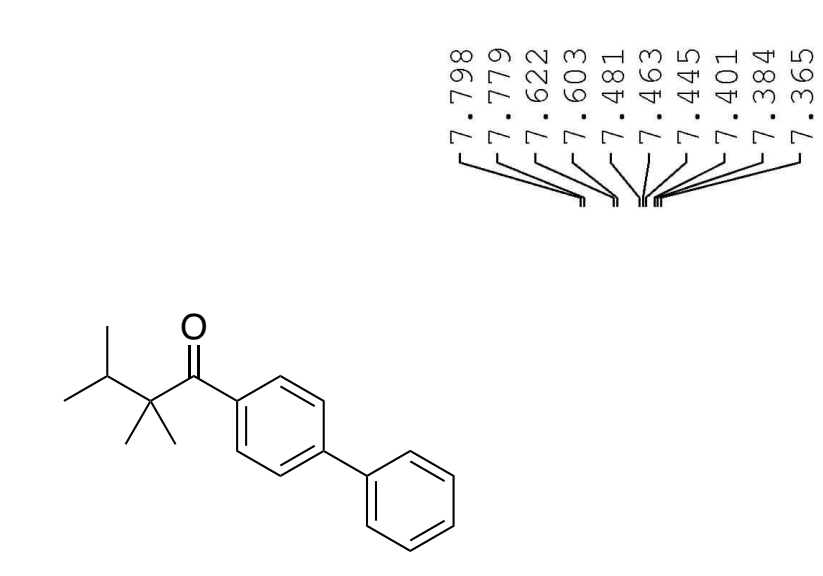

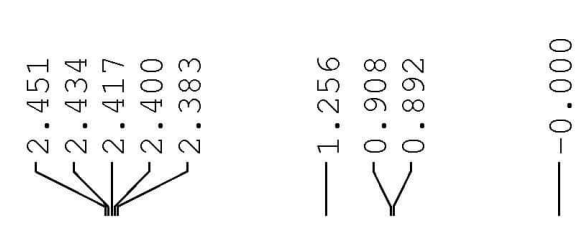

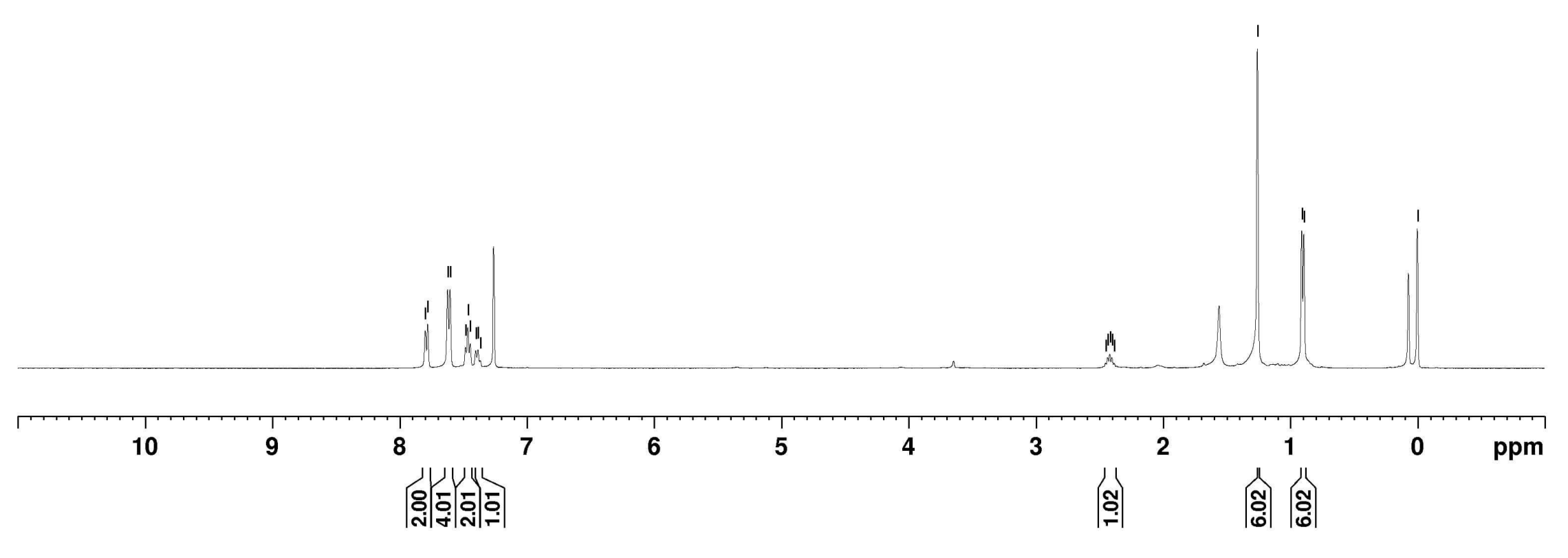

S52 


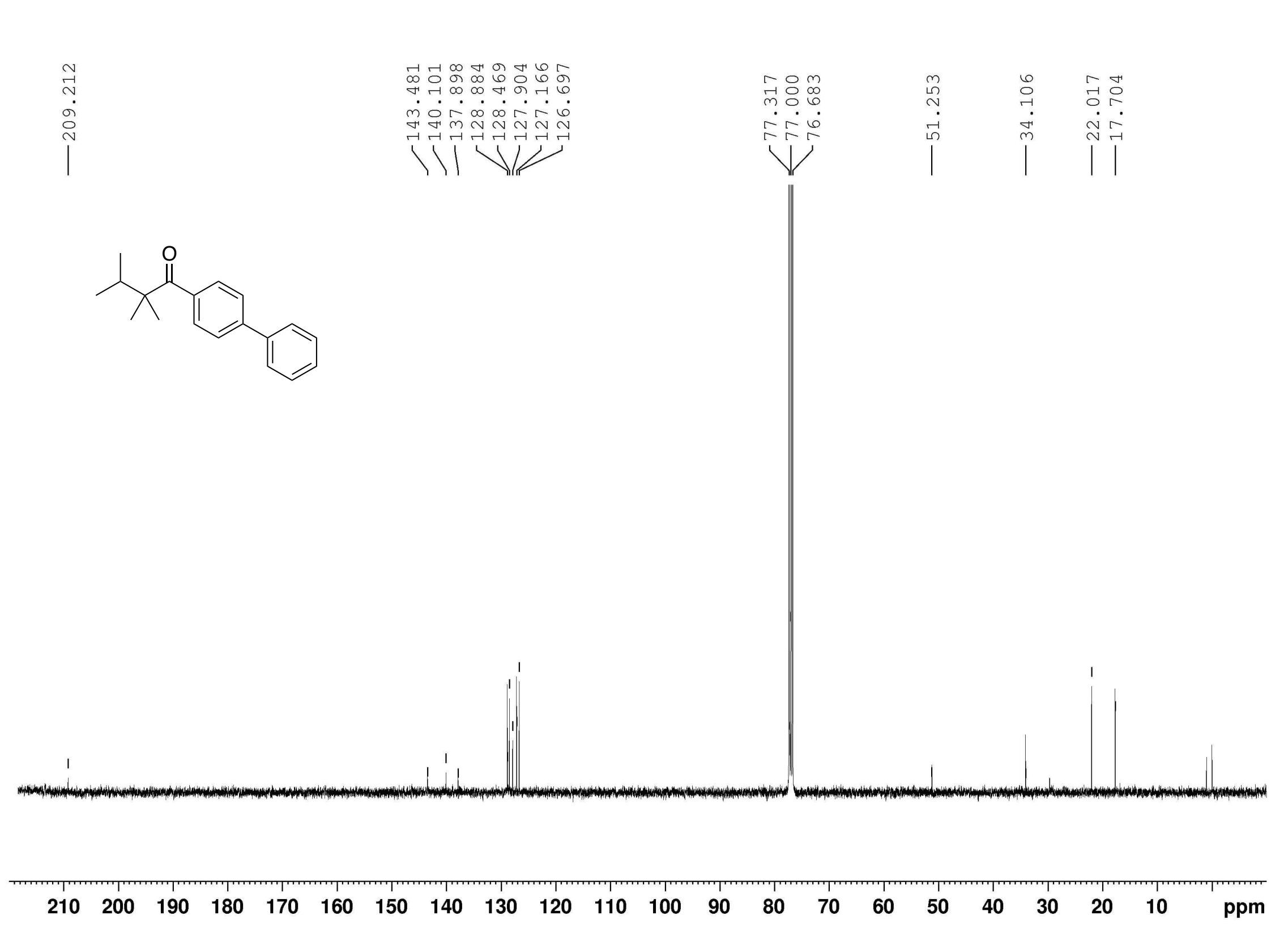


${ }^{1} \mathrm{H}$ NMR of $\mathbf{4 c b}$
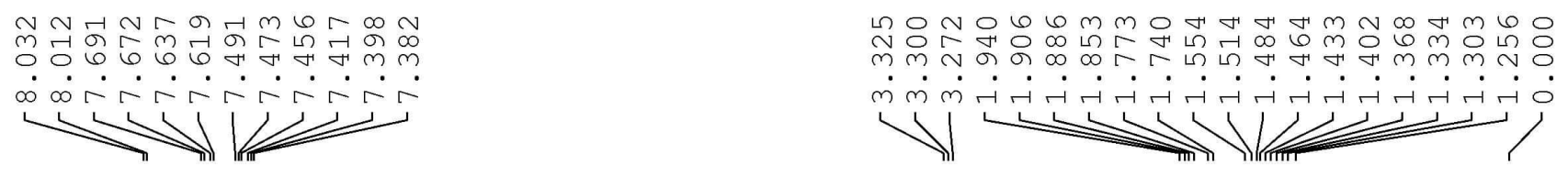<smiles>O=C(c1ccc(-c2ccccc2)cc1)C1CCCCC1</smiles>

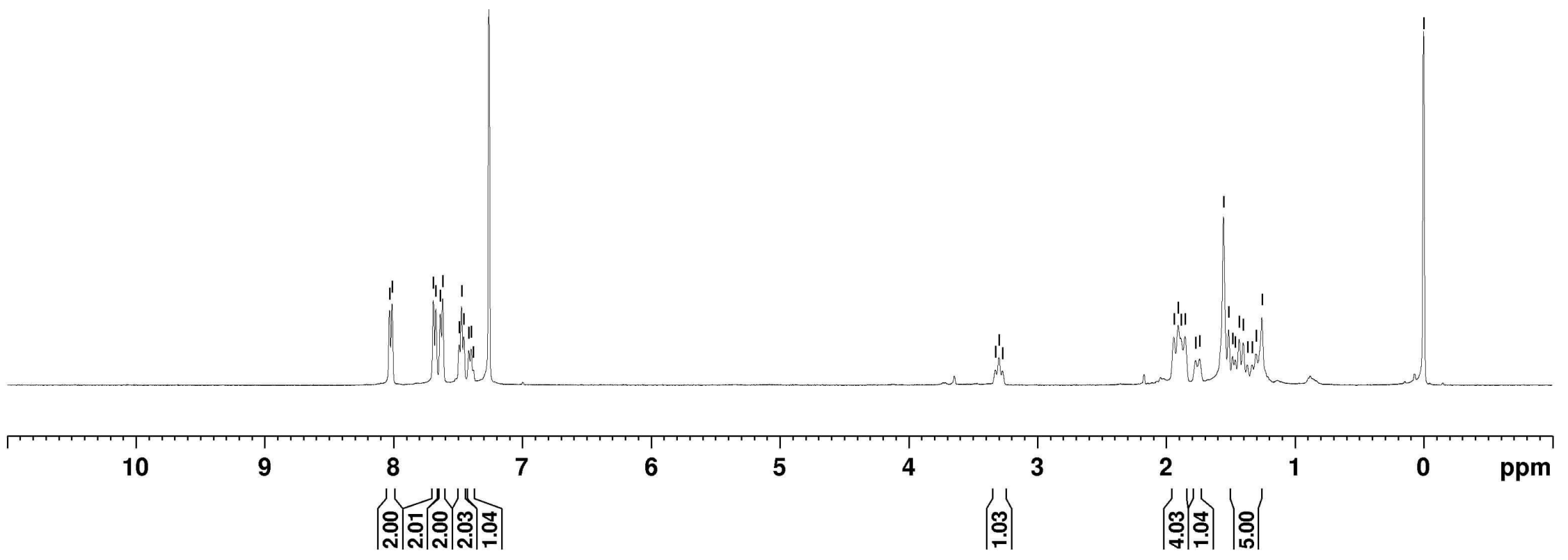

S54 
${ }^{13} \mathrm{C}$ NMR of $4 \mathrm{cb}$

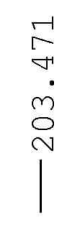<smiles>O=C(c1ccc(-c2ccccc2)cc1)C1CCCCC1</smiles>

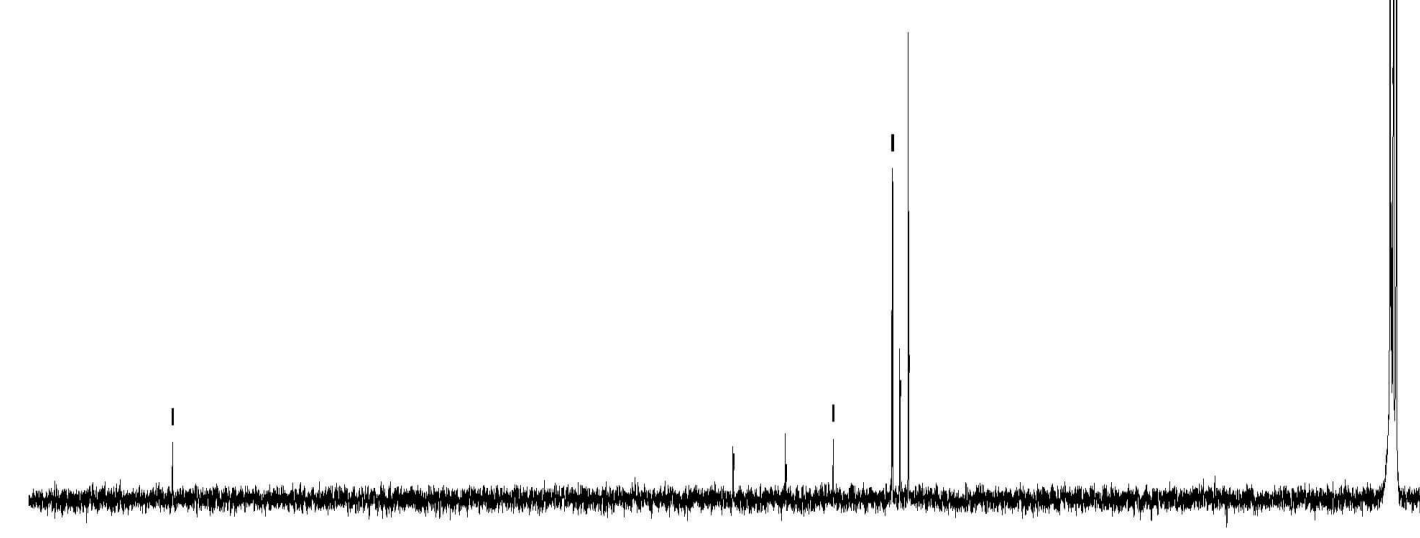

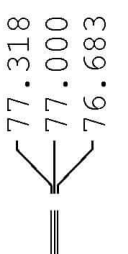

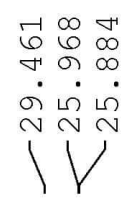

$\begin{array}{llllllllllllllll}210 & 200 & 190 & 180 & 170 & 160 & 150 & 140 & 130 & 120 & 110 & 100 & 90 & 80 & 70\end{array}$

60

50

40

30

20

10

ppm

S55 


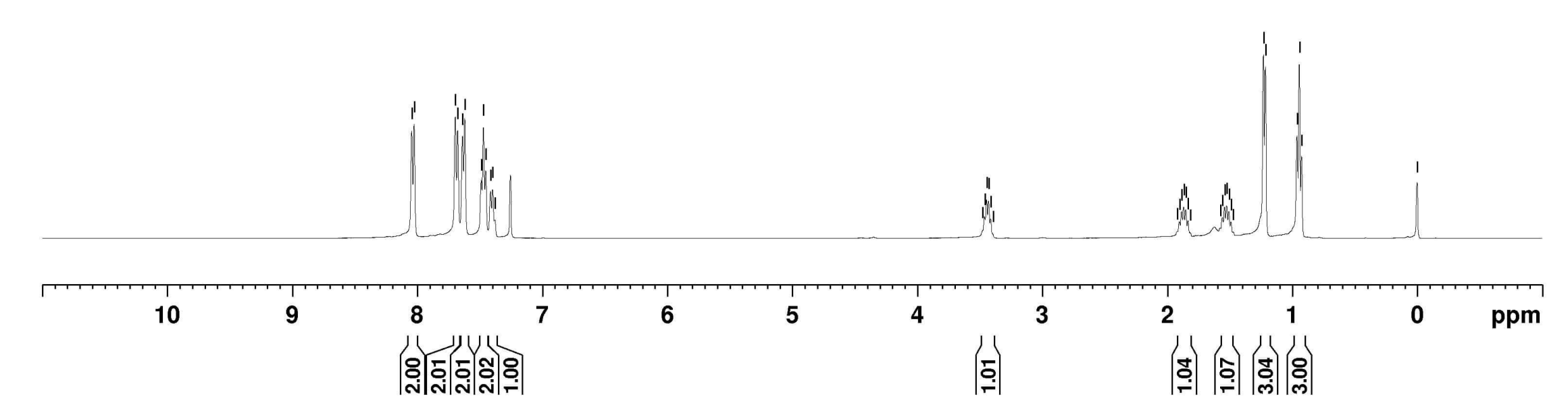


${ }^{13} \mathrm{C}$ NMR of $\mathbf{4 d b}$

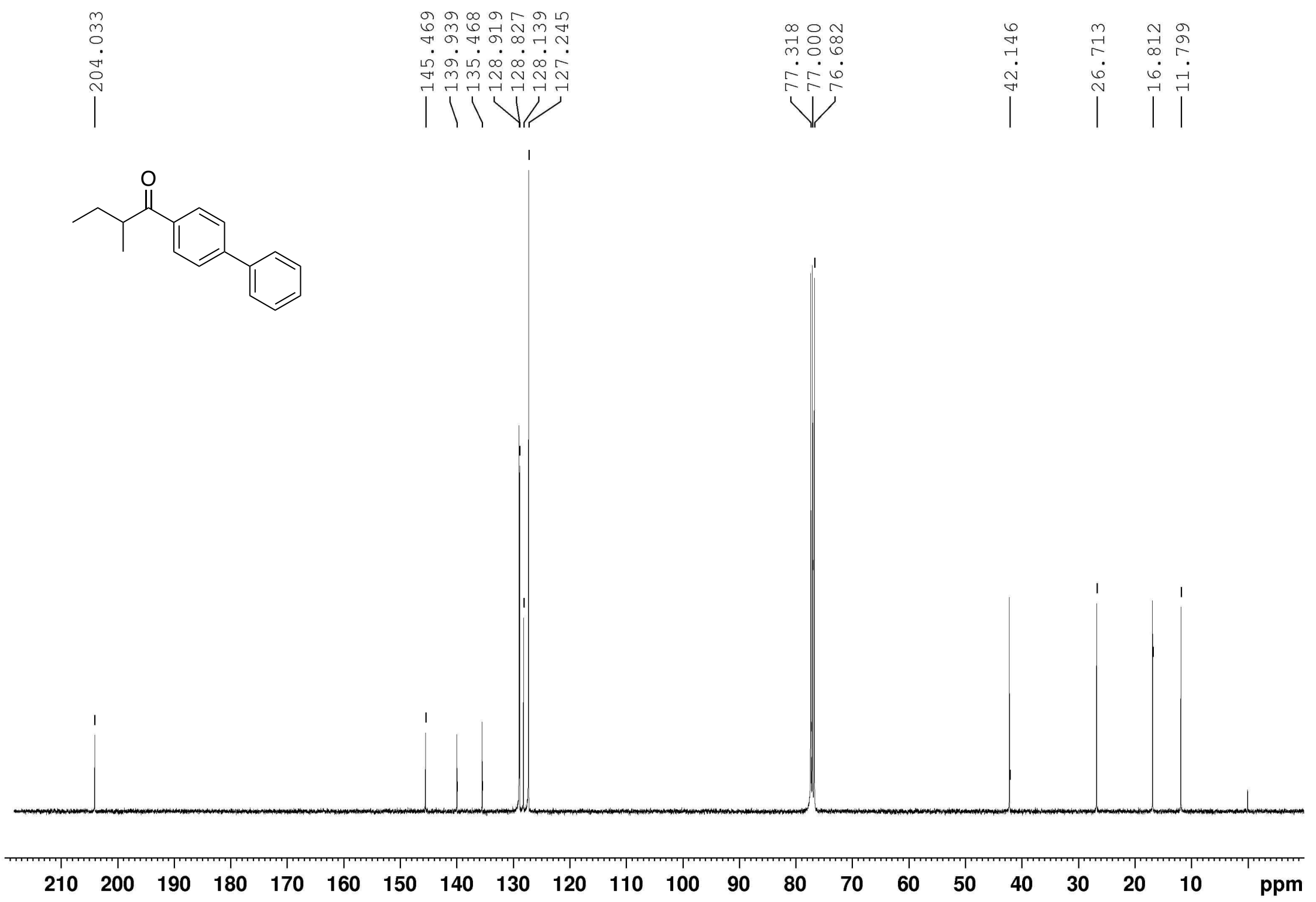

S57 


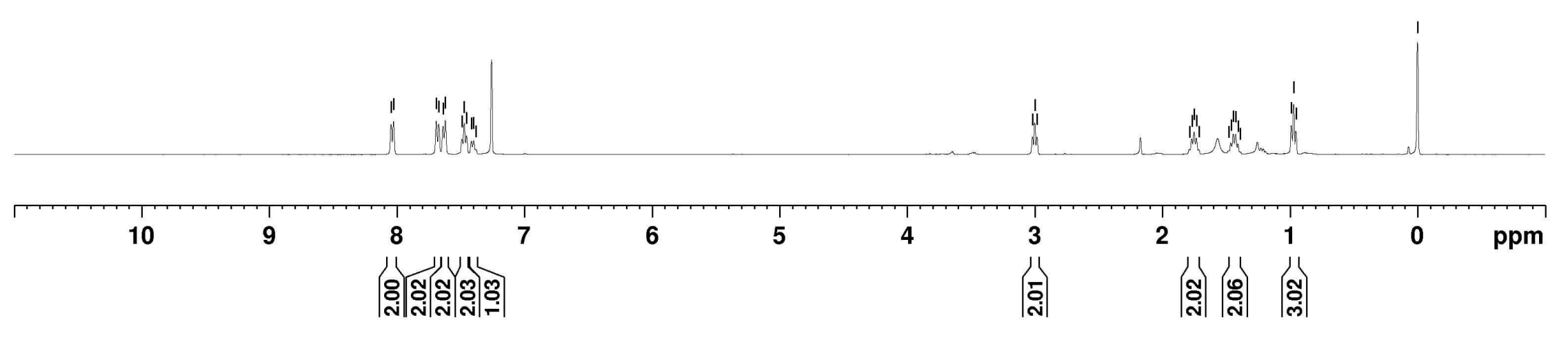


${ }^{13} \mathrm{C}$ NMR of $4 \mathbf{e b}$
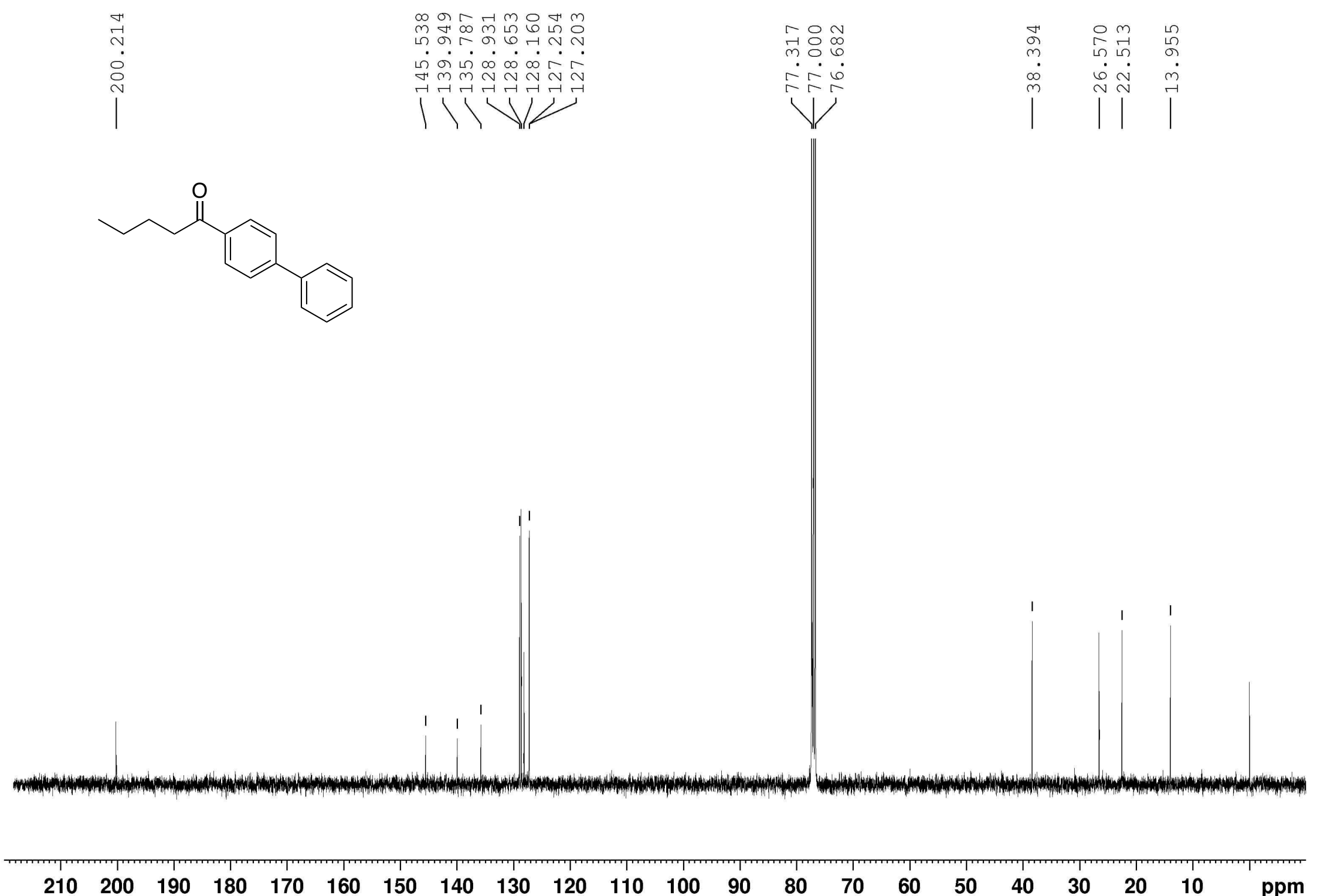

50

$40 \quad 30$

20 
${ }^{1} \mathrm{H}$ NMR of $\mathbf{4 f b}$
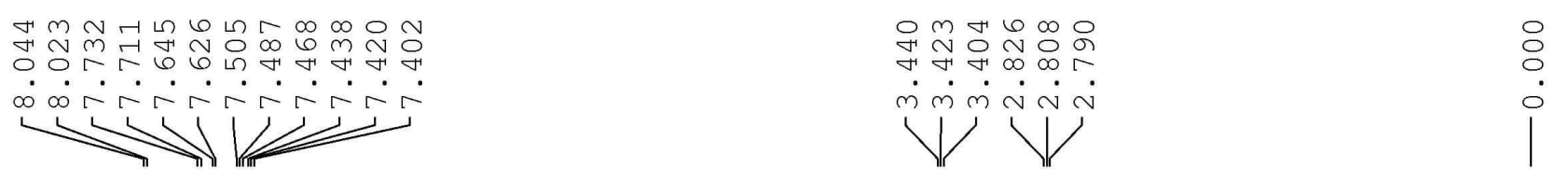<smiles>N#CCCC(=O)c1ccc(-c2ccccc2)cc1</smiles>
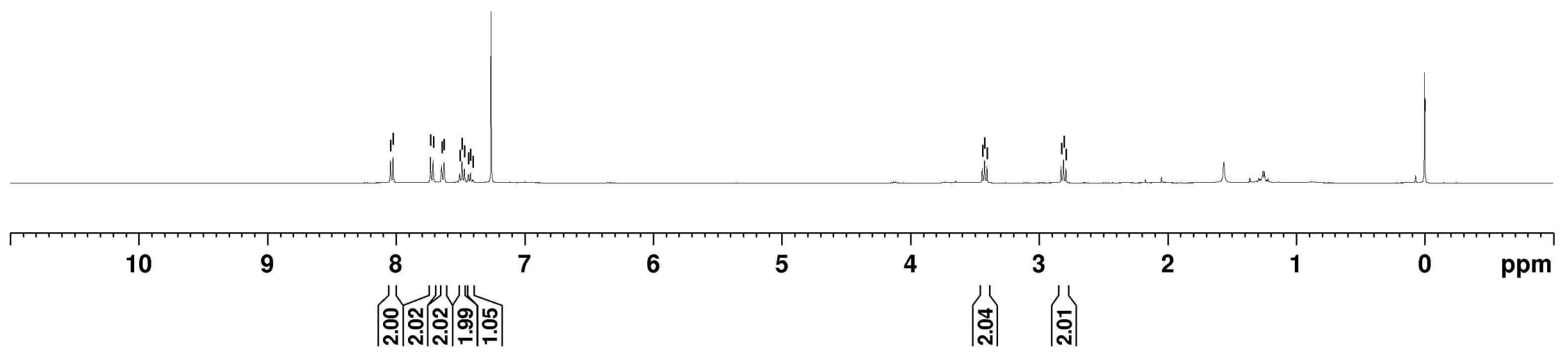

S60 
${ }^{13} \mathrm{C}$ NMR of $\mathbf{4 f b}$
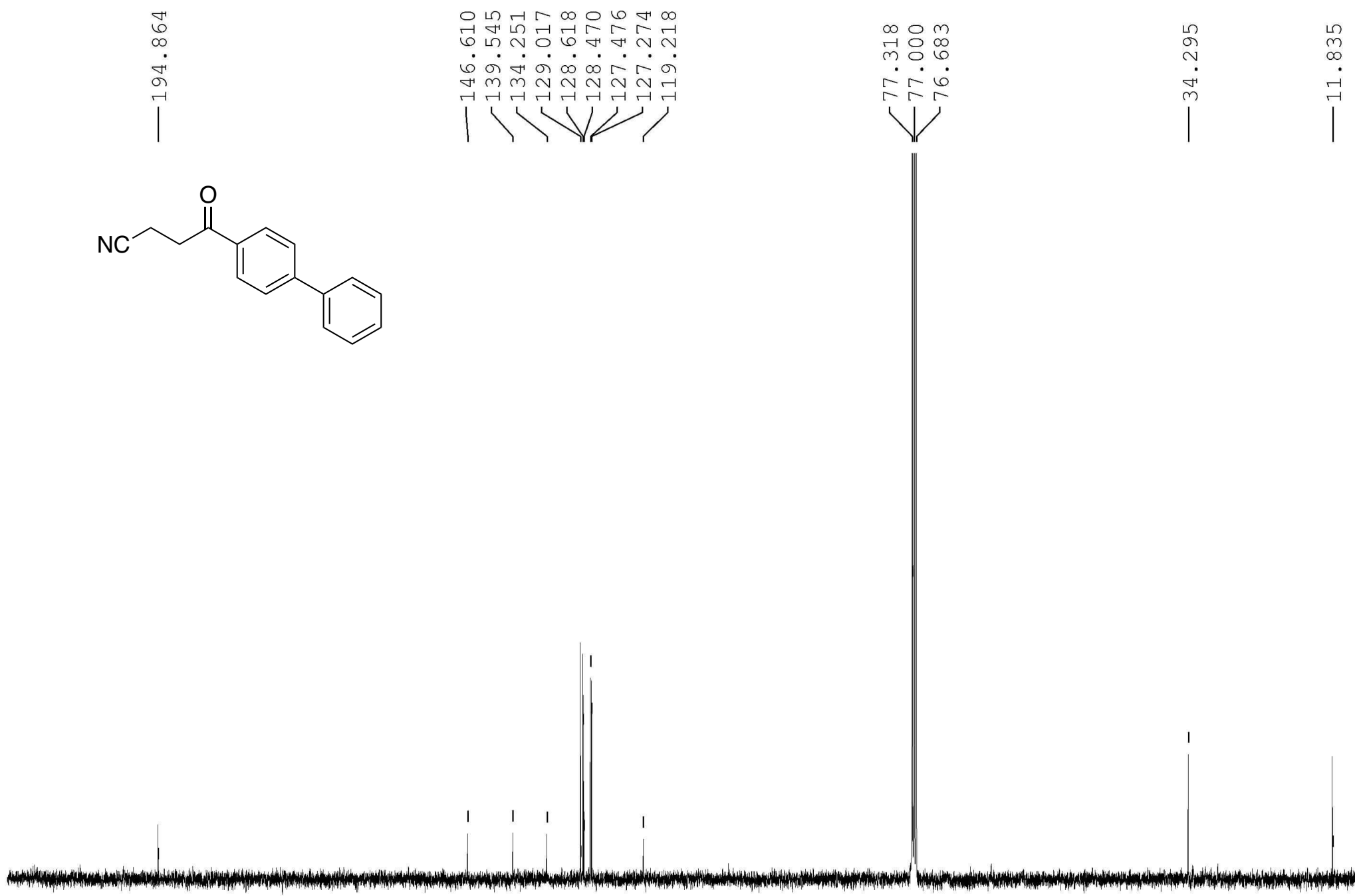

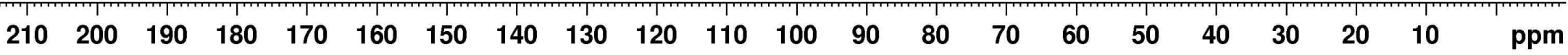

S61 
${ }^{1} \mathrm{H}$ NMR of $\mathbf{4 g b}$
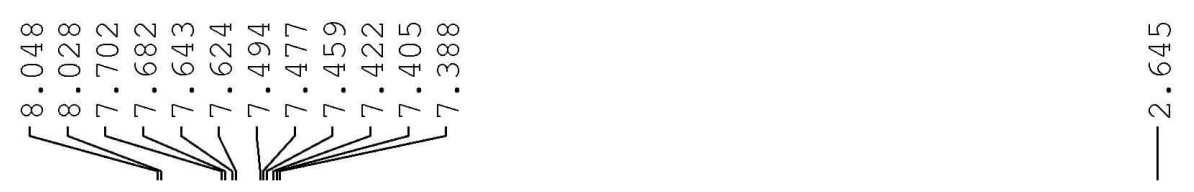

$\circ$
$\vdots$
0
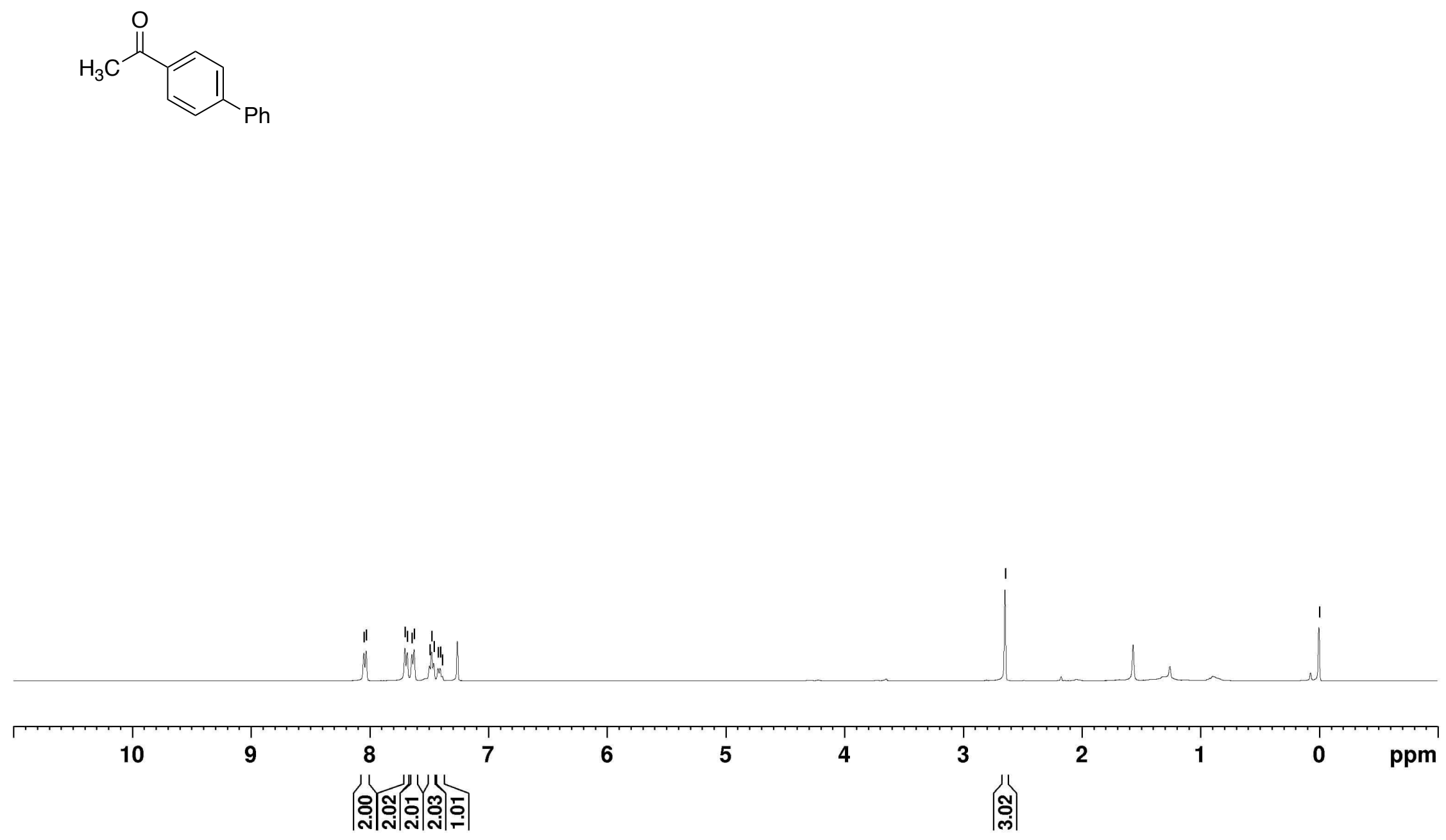

S62 
${ }^{13} \mathrm{C}$ NMR of $4 \mathbf{g b}$
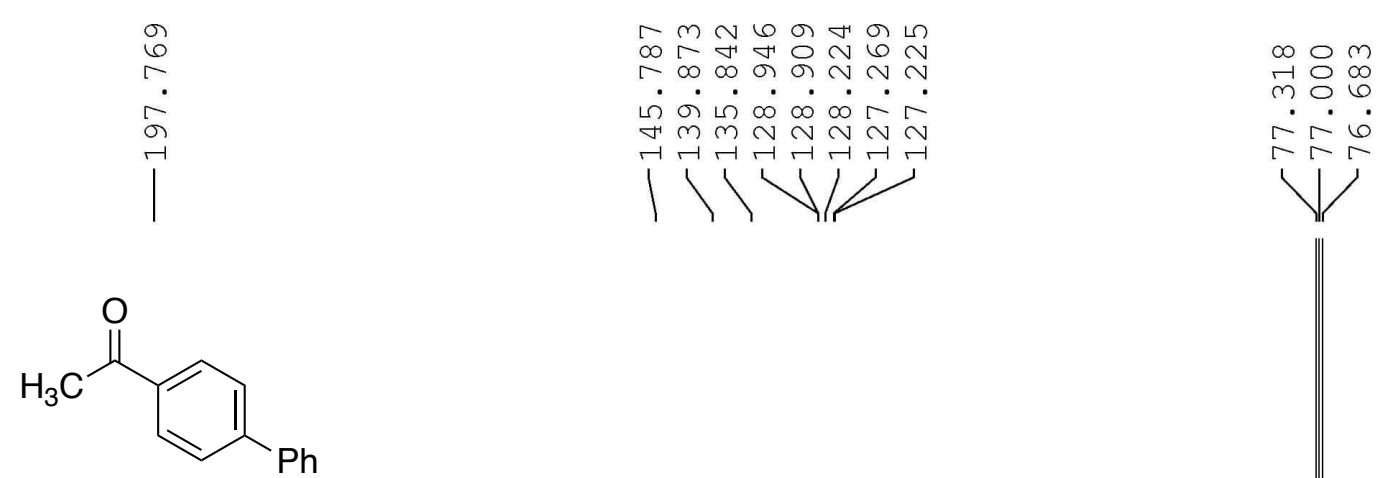

0
$\stackrel{0}{0}$
$\stackrel{0}{N}$

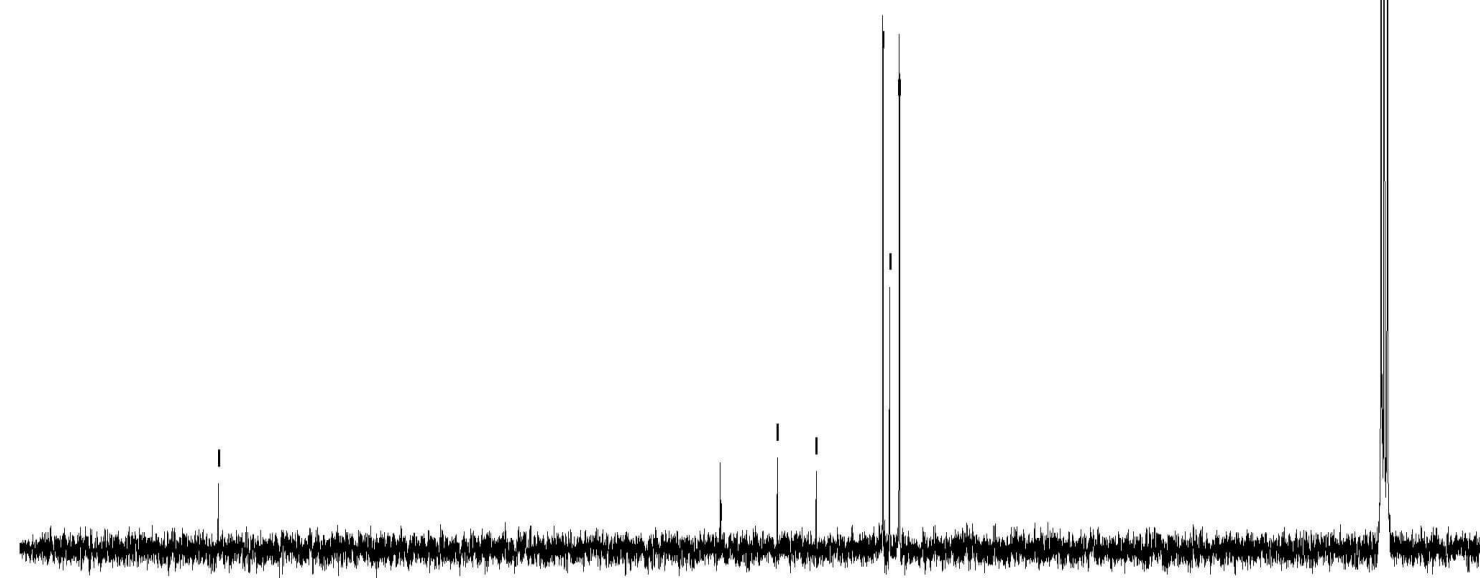

$\begin{array}{lllllllll}210 & 200 & 190 & 180 & 170 & 160 & 150 & 140 & 130\end{array}$

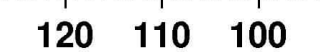

$90 \quad 80$

8070

60

50

$40 \quad 30$

20

10

ppm 
${ }^{1} \mathrm{H}$ NMR of $[2 \mathrm{H}] \mathbf{4 g b}$
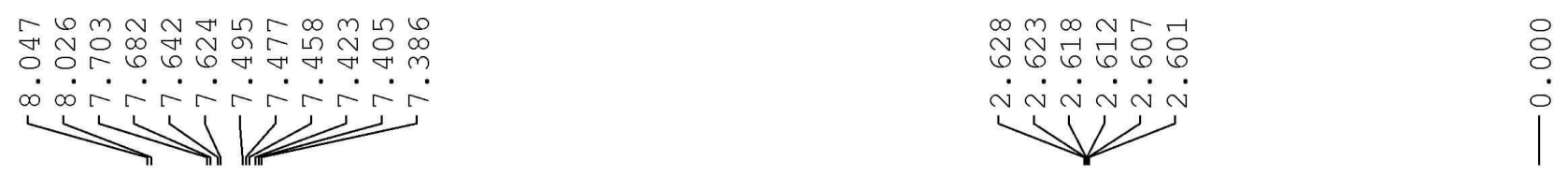<smiles>[2H][C+]([O-])C(=O)c1ccc(-c2ccccc2)cc1</smiles>

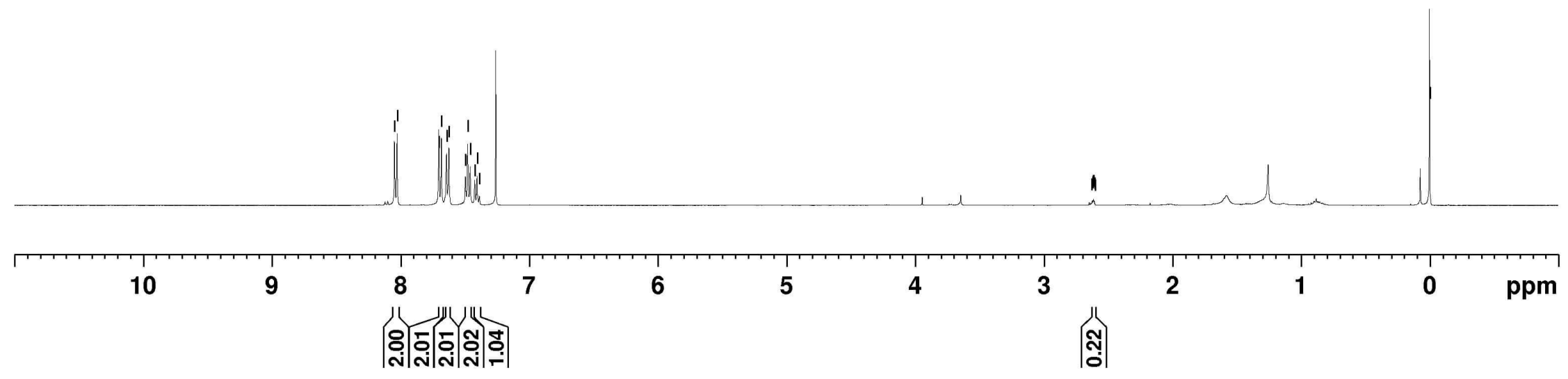

S64 

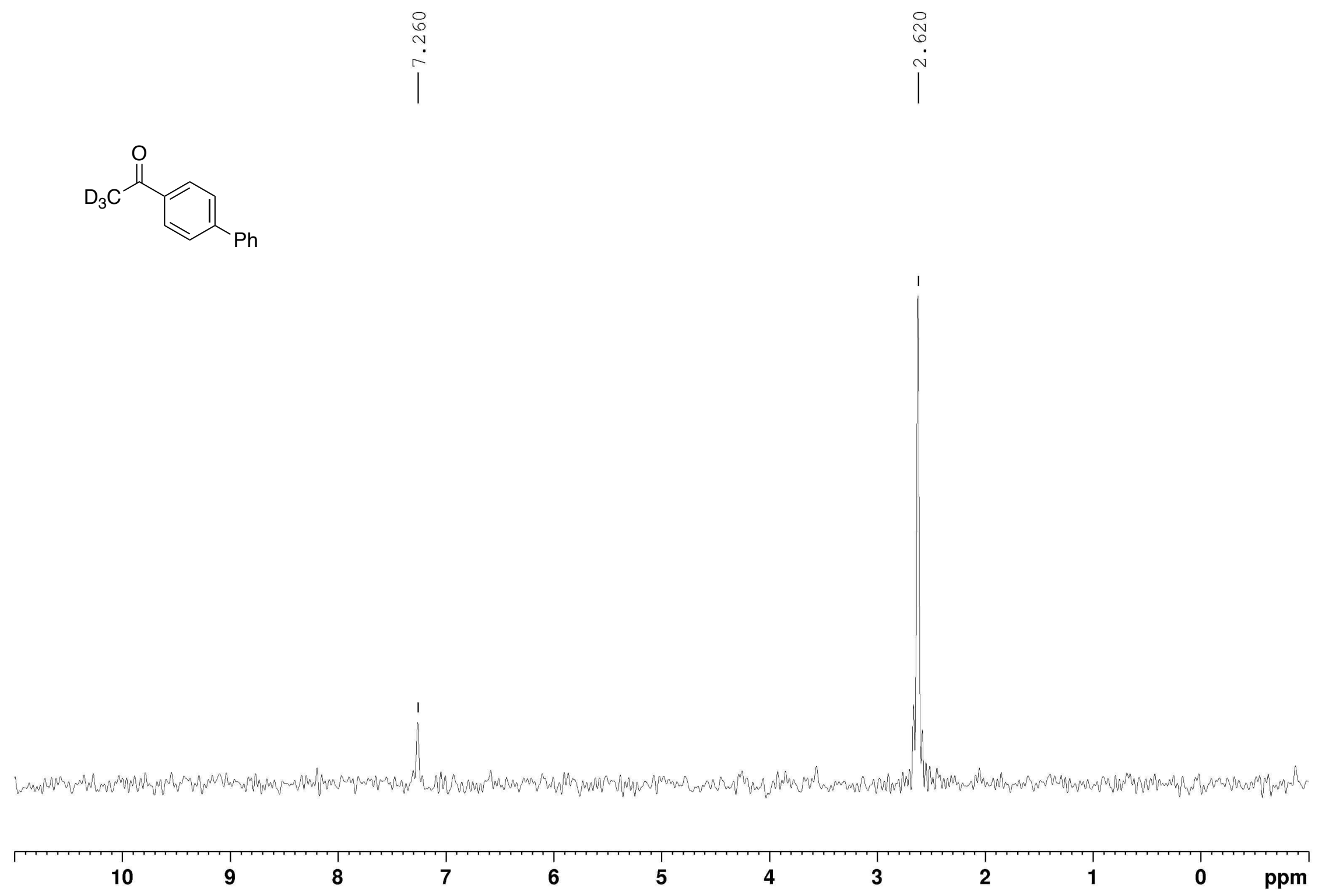

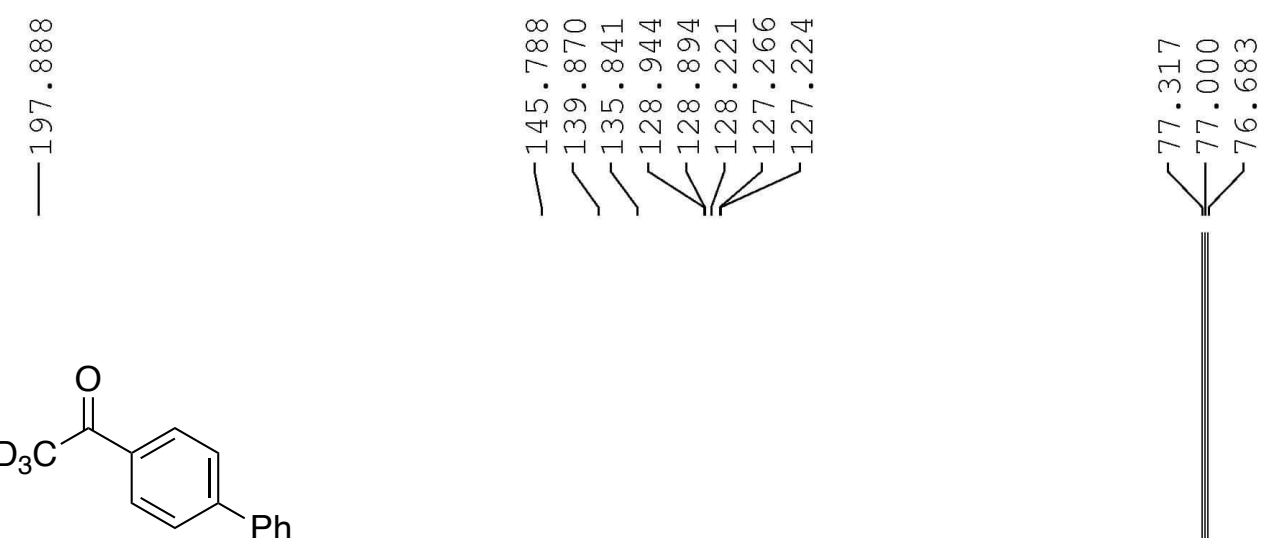

กิ

ले

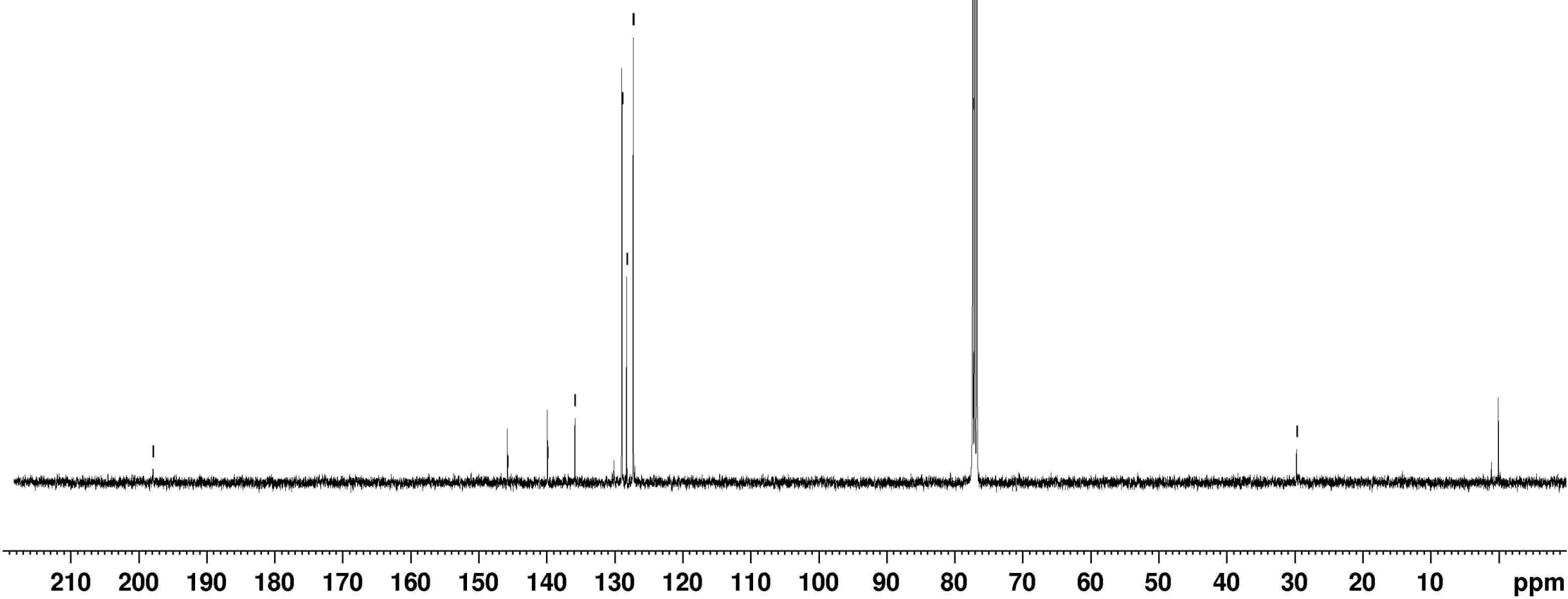



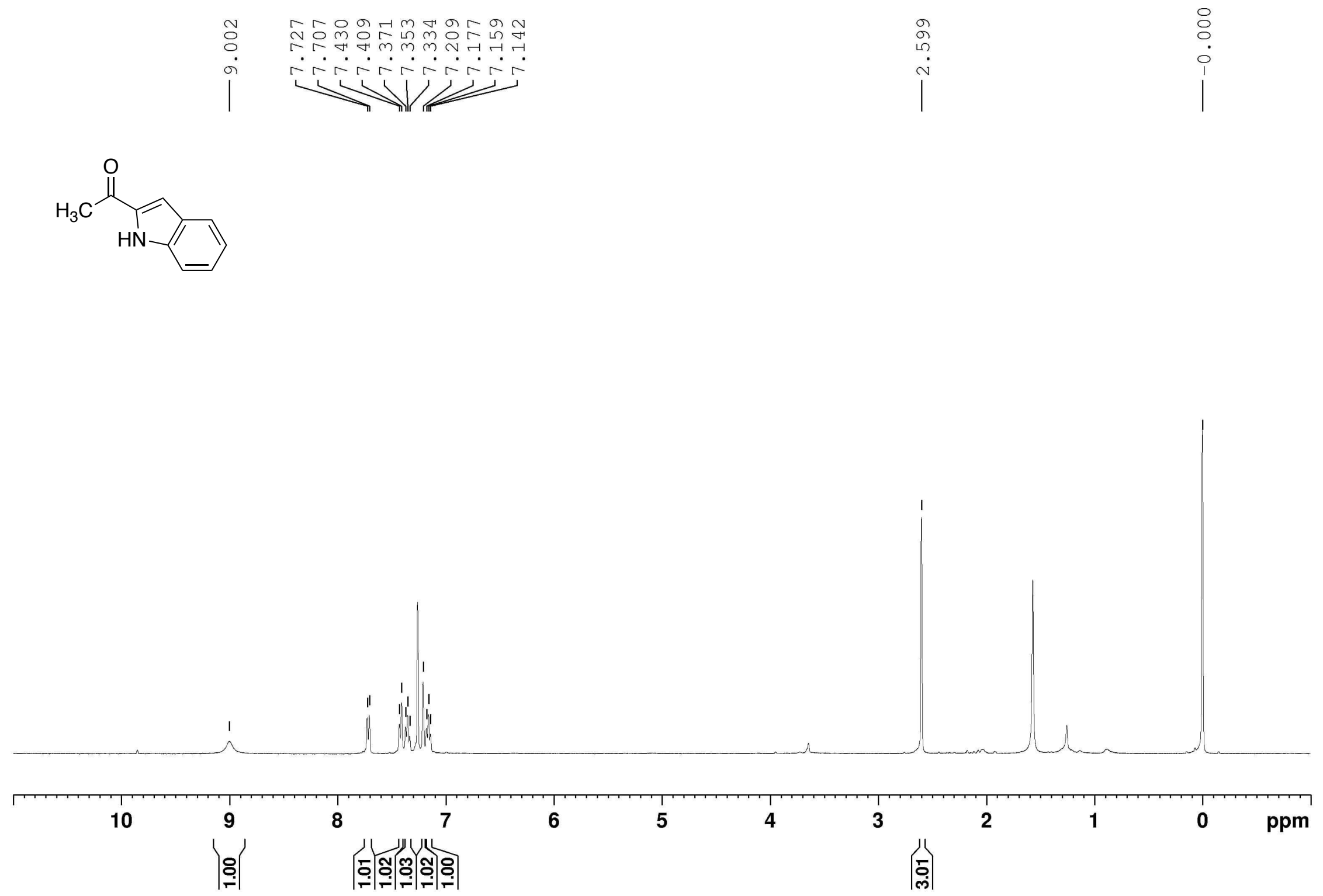


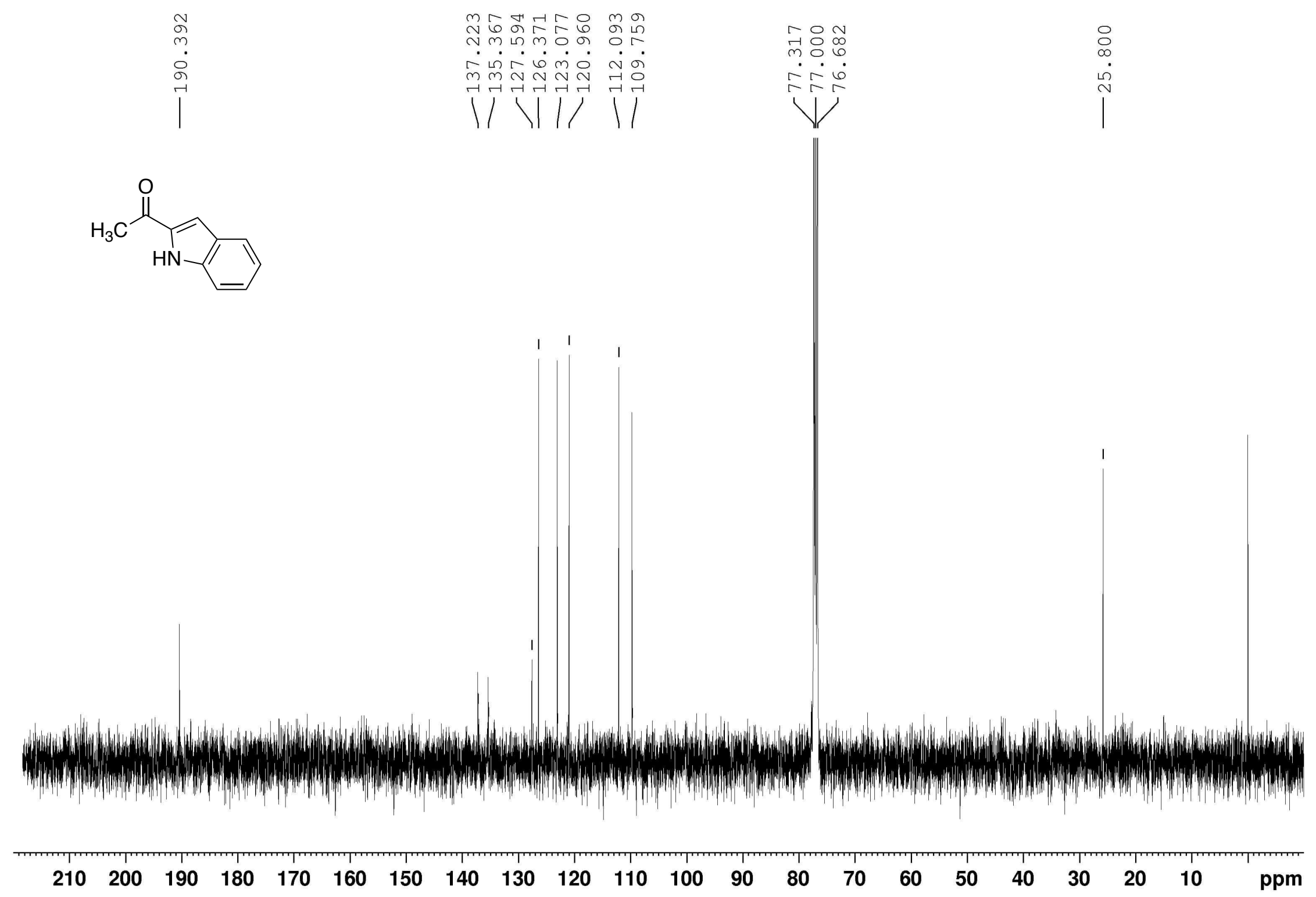



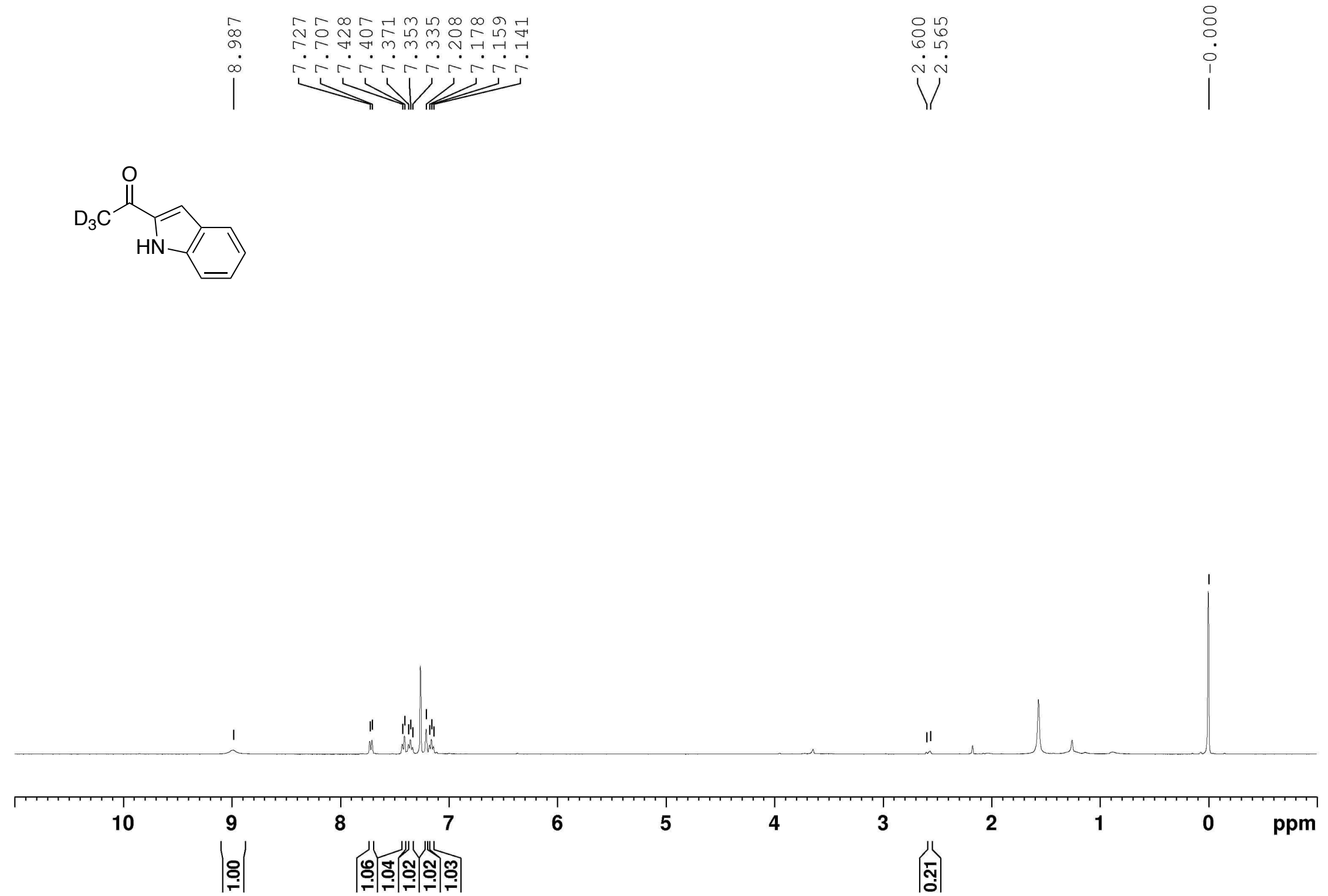

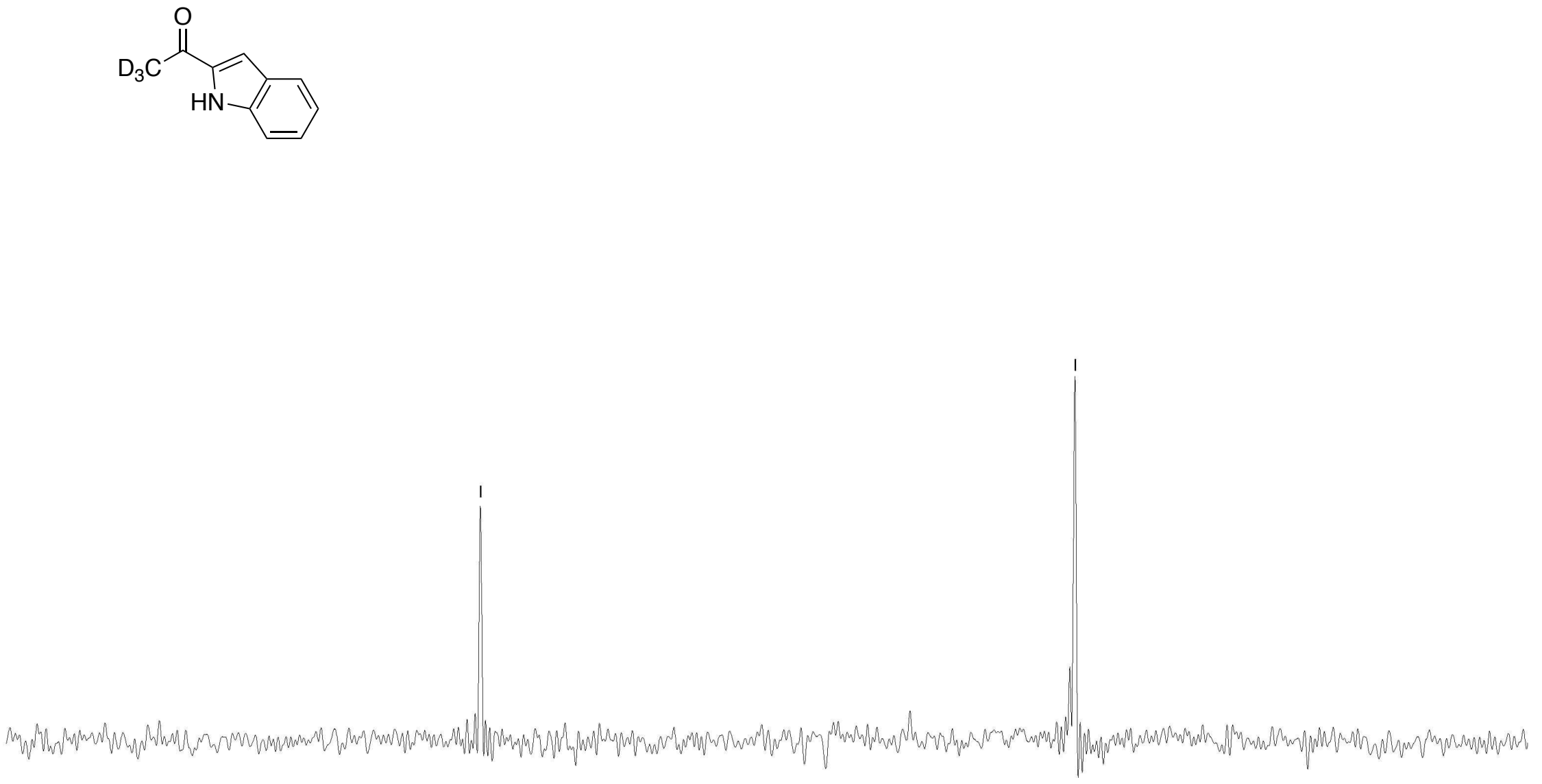

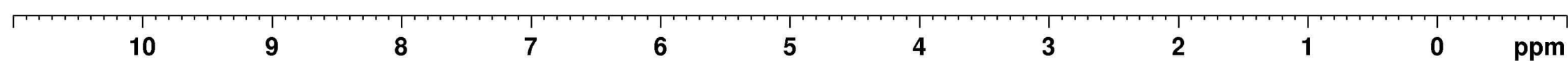




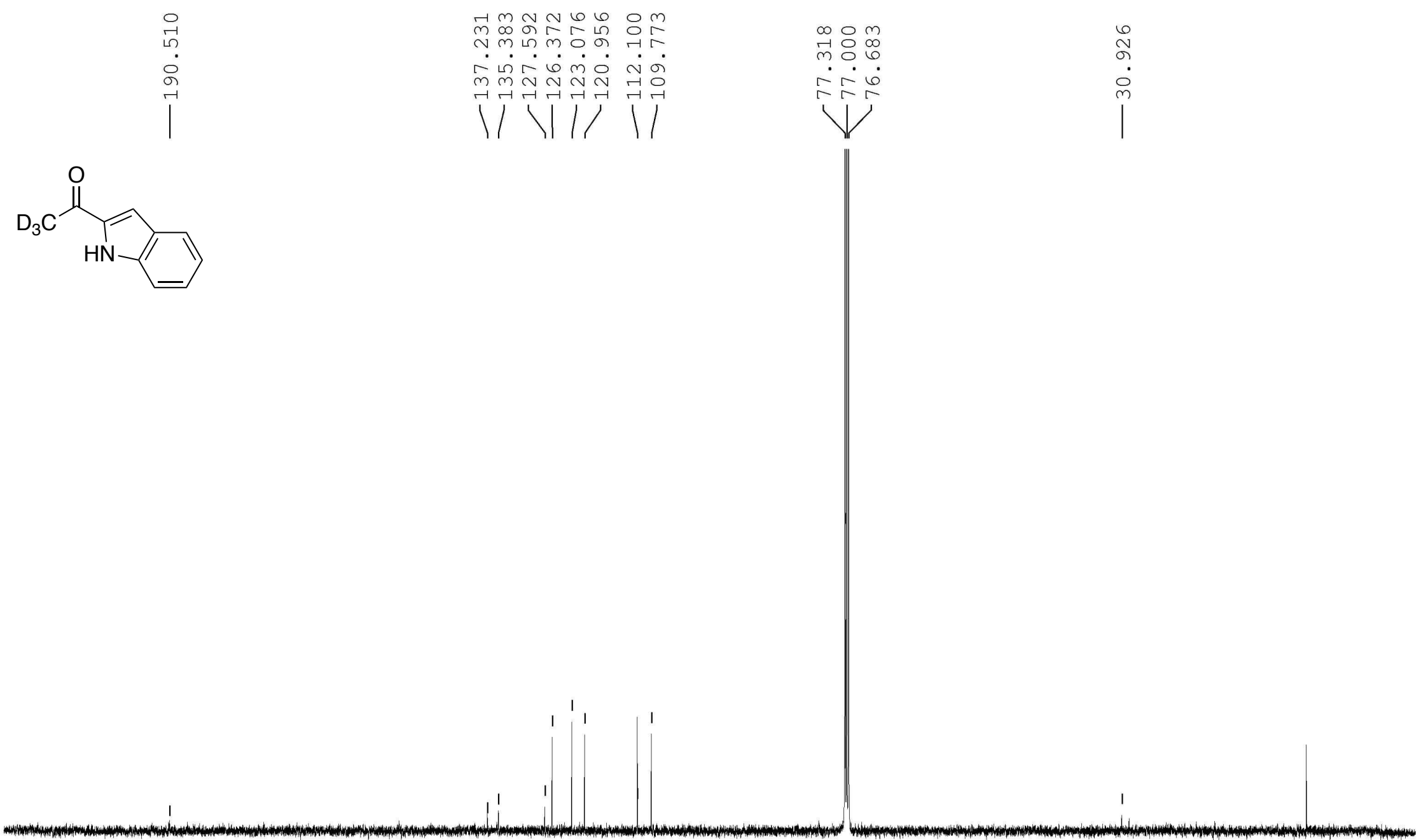

$\begin{array}{lllllllllllllllllllllll}210 & 200 & 190 & 180 & 170 & 160 & 150 & 140 & 130 & 120 & 110 & 100 & 90 & 80 & 70 & 60 & 50 & 40 & 30 & 20 & 10 & 0 & \mathrm{ppm}\end{array}$ 


\section{${ }^{1} \mathrm{H}$ NMR of $\mathbf{4 g d}$}
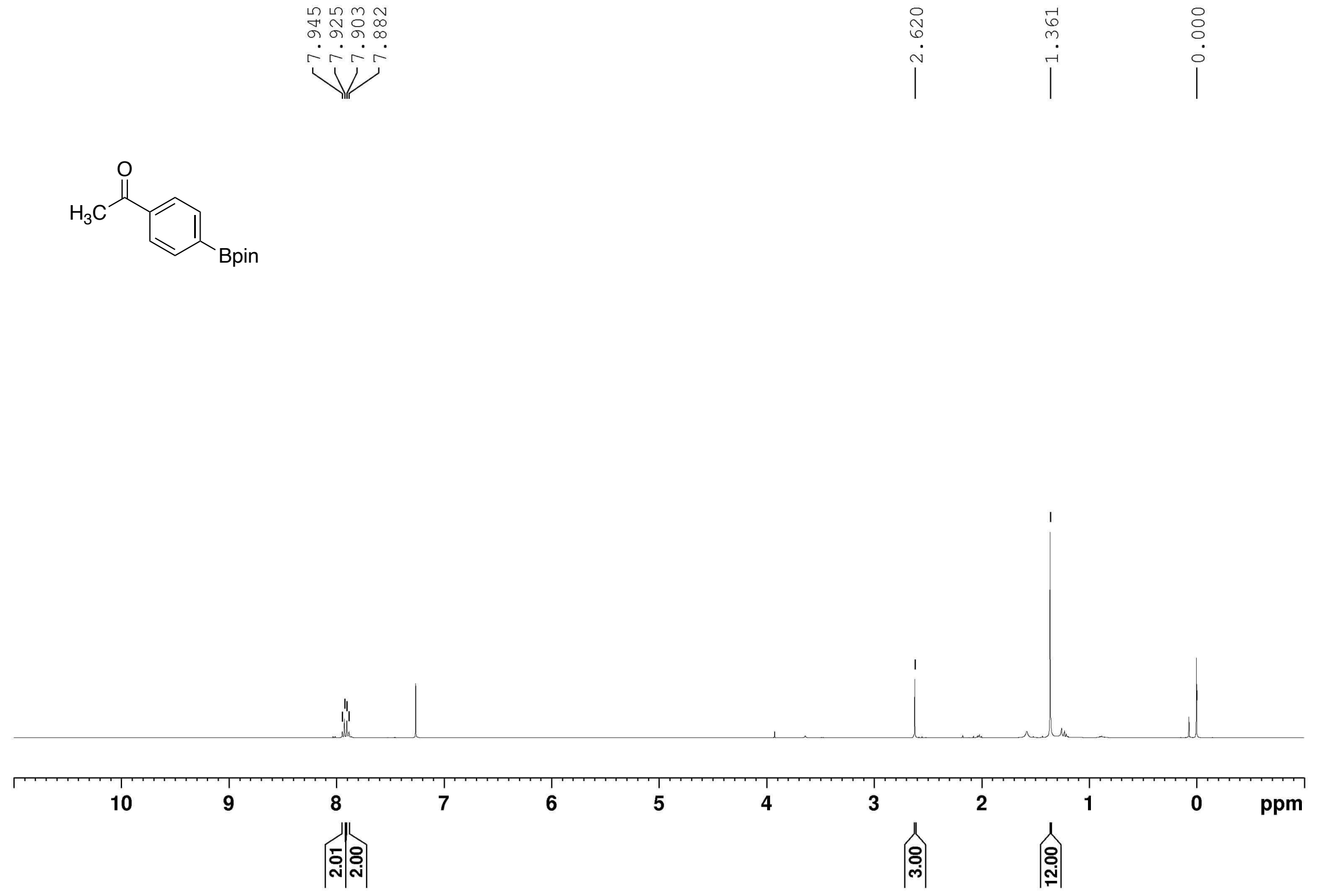
${ }^{13} \mathrm{C}$ NMR of $4 \mathbf{g d}$
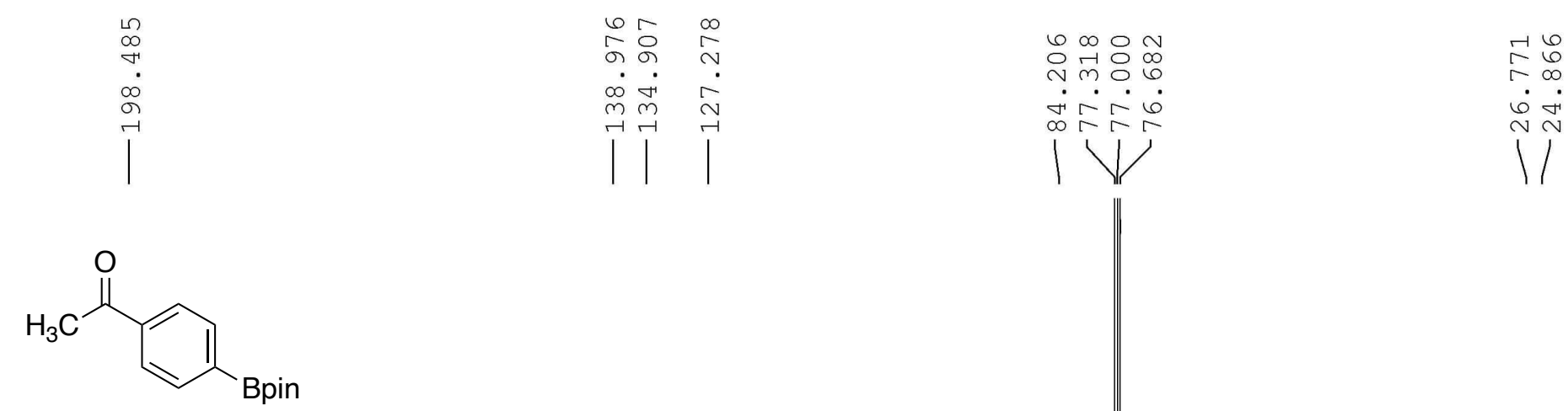

Bpin

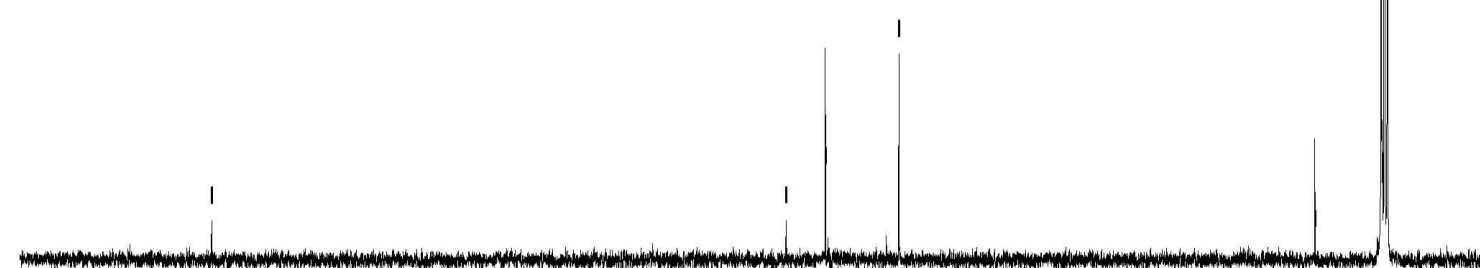

$\begin{array}{lllllllllllllllllllllll}210 & 200 & 190 & 180 & 170 & 160 & 150 & 140 & 130 & 120 & 110 & 100 & 90 & 80 & 70 & 60 & 50 & 40 & 30 & 20 & 10 & \mathrm{ppm}\end{array}$ 


\section{${ }^{11} \mathrm{~B}$ NMR of $\mathbf{4 g d}$}
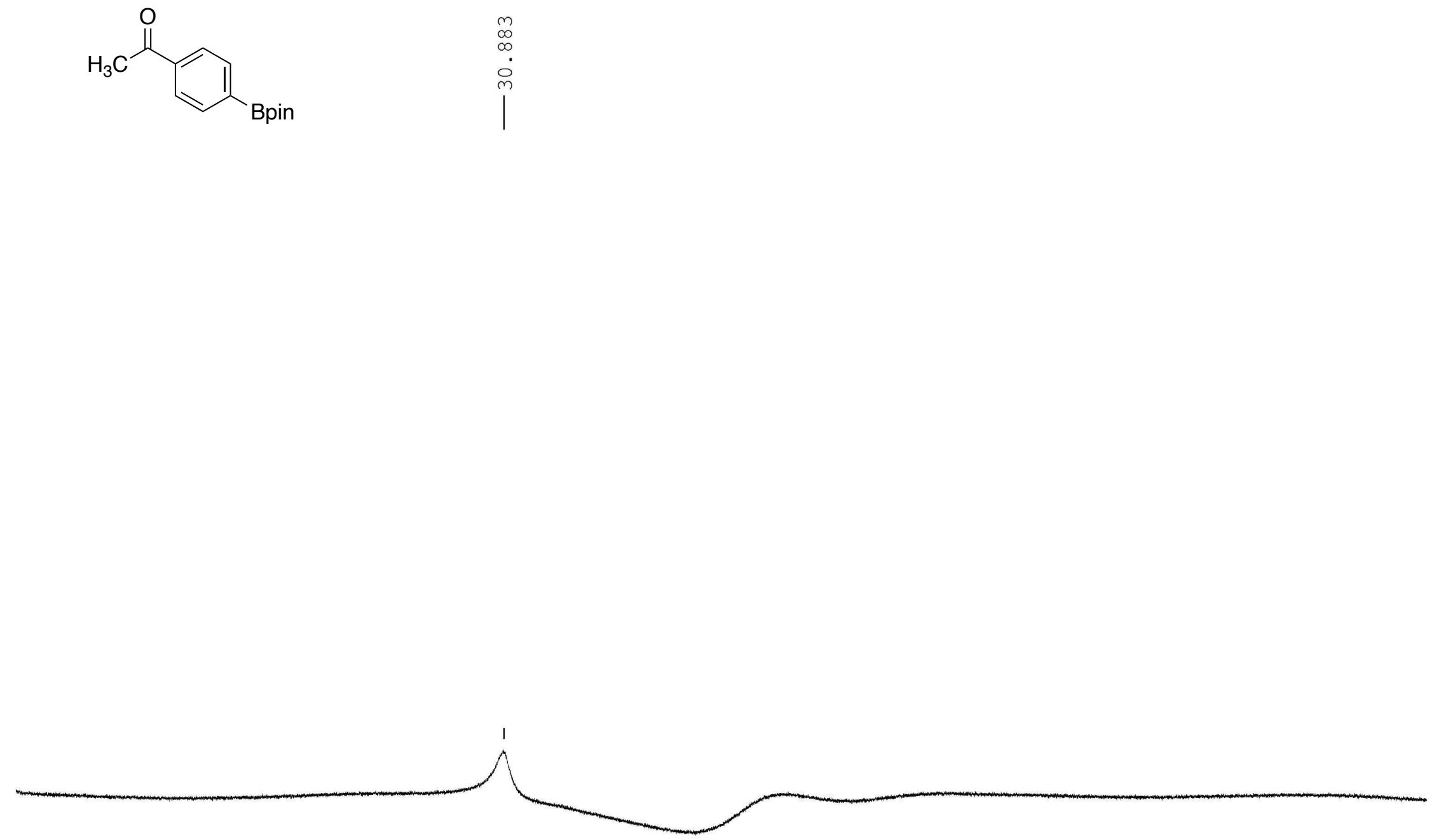
${ }^{1} \mathrm{H}$ NMR of $[2 \mathrm{H}] \mathbf{4 g d}$
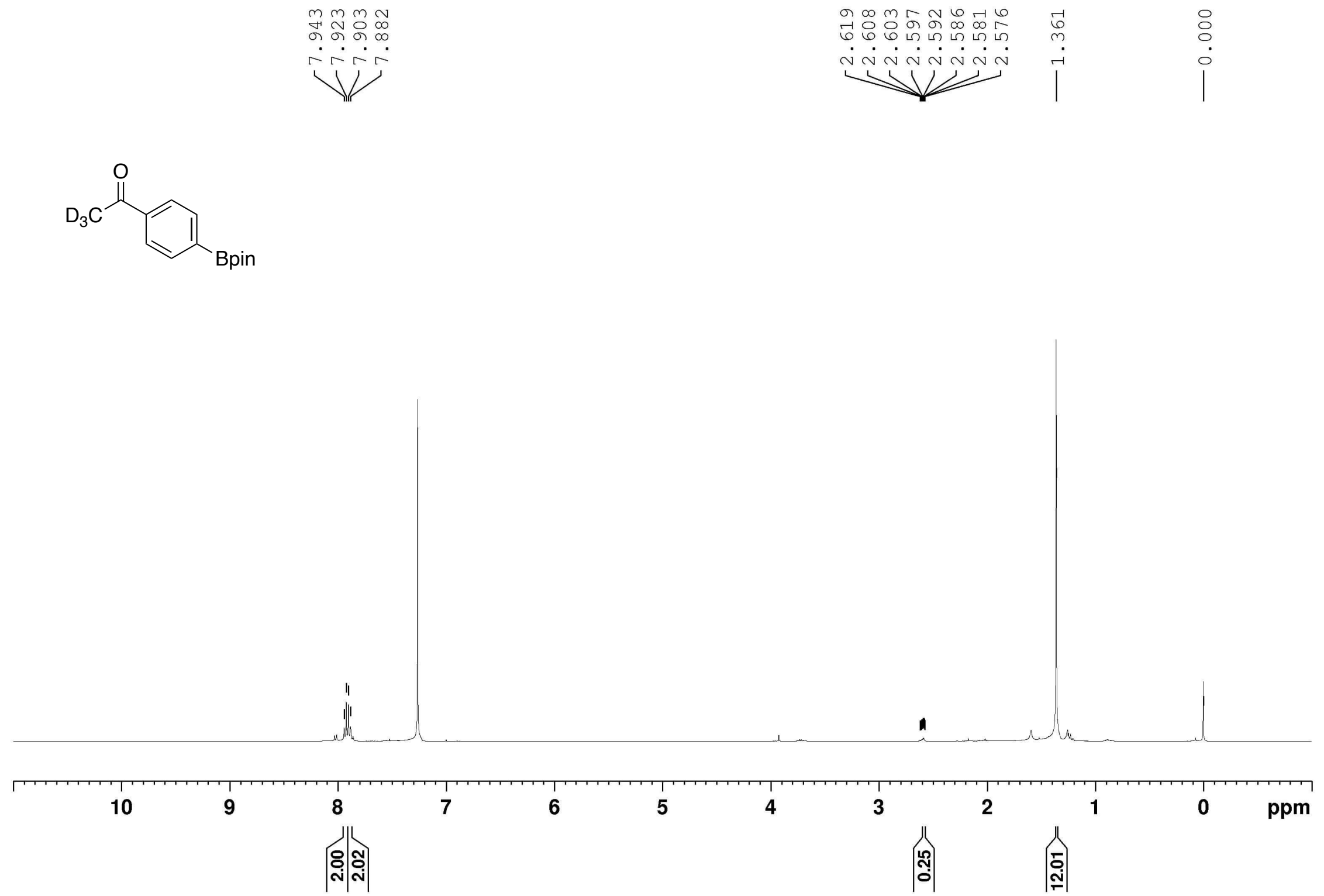
${ }^{2} \mathrm{H}$ NMR of $[2 \mathrm{H}] \mathbf{4 g d}$
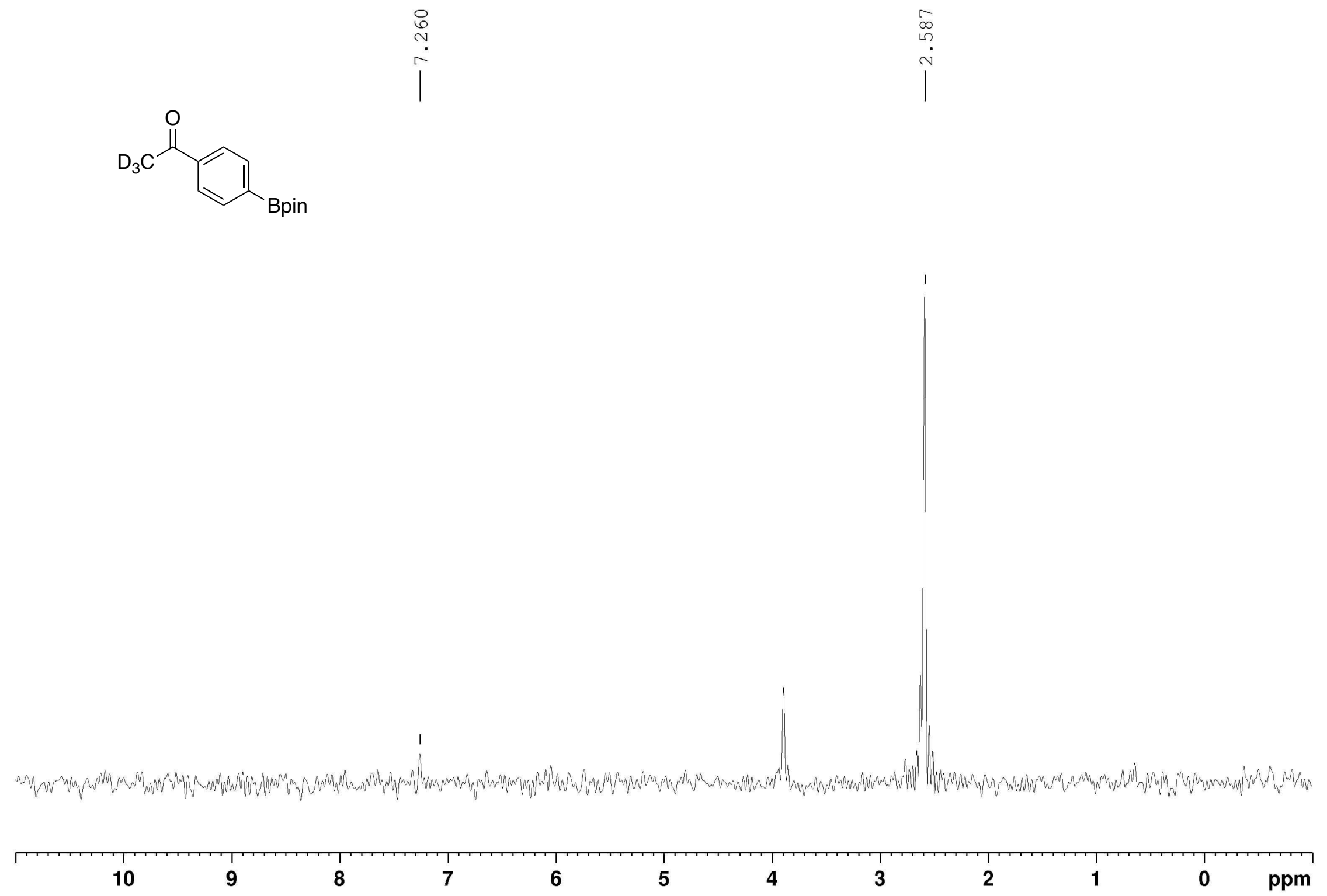

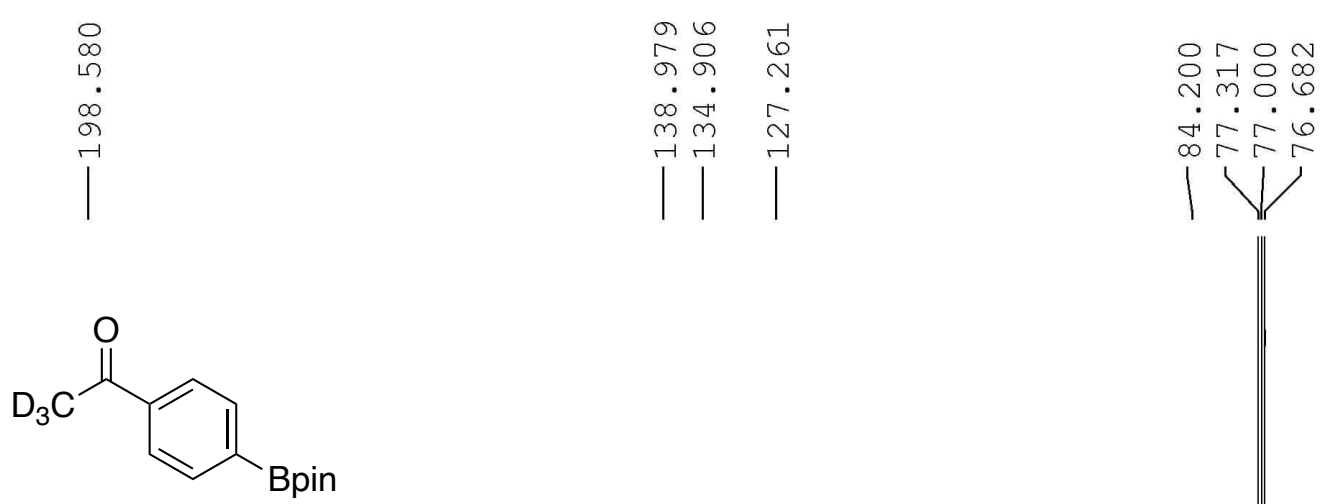

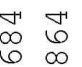

홈
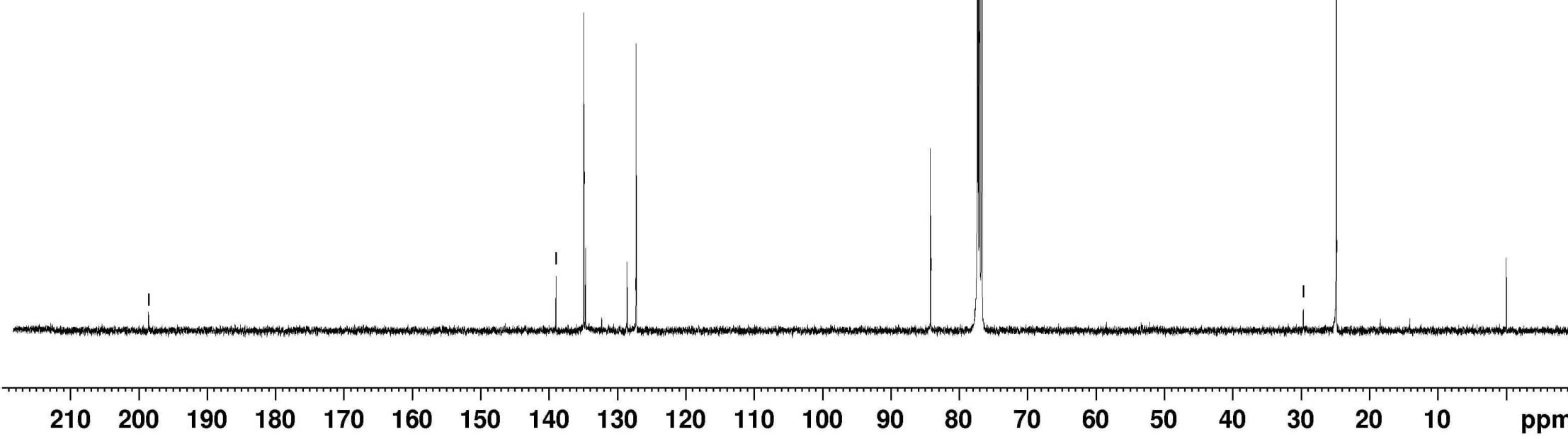
${ }^{11} \mathrm{~B}$ NMR of $[2 \mathrm{H}] \mathbf{4 g d}$
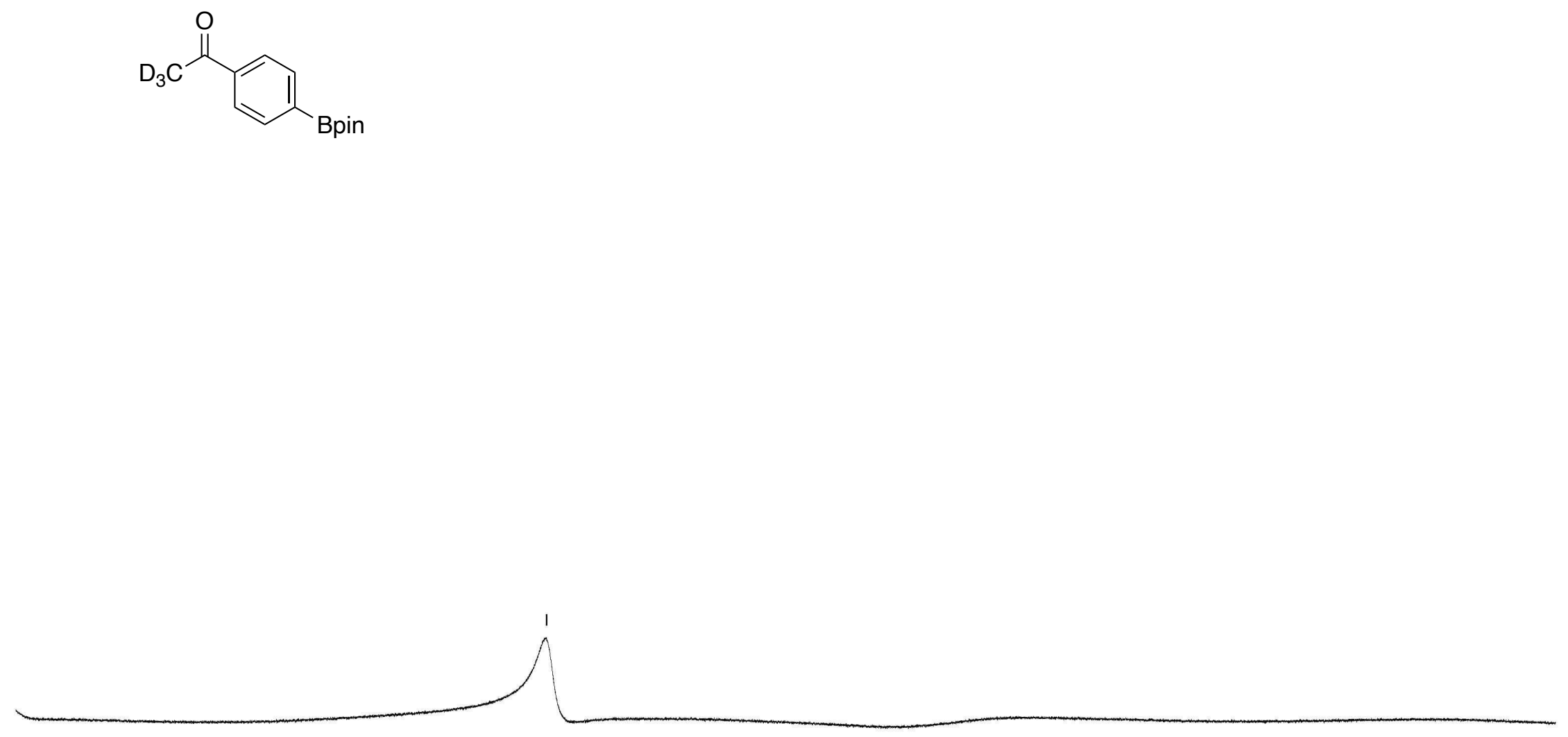


\section{${ }^{1} \mathrm{H}$ NMR of $\mathbf{4 a e}$}
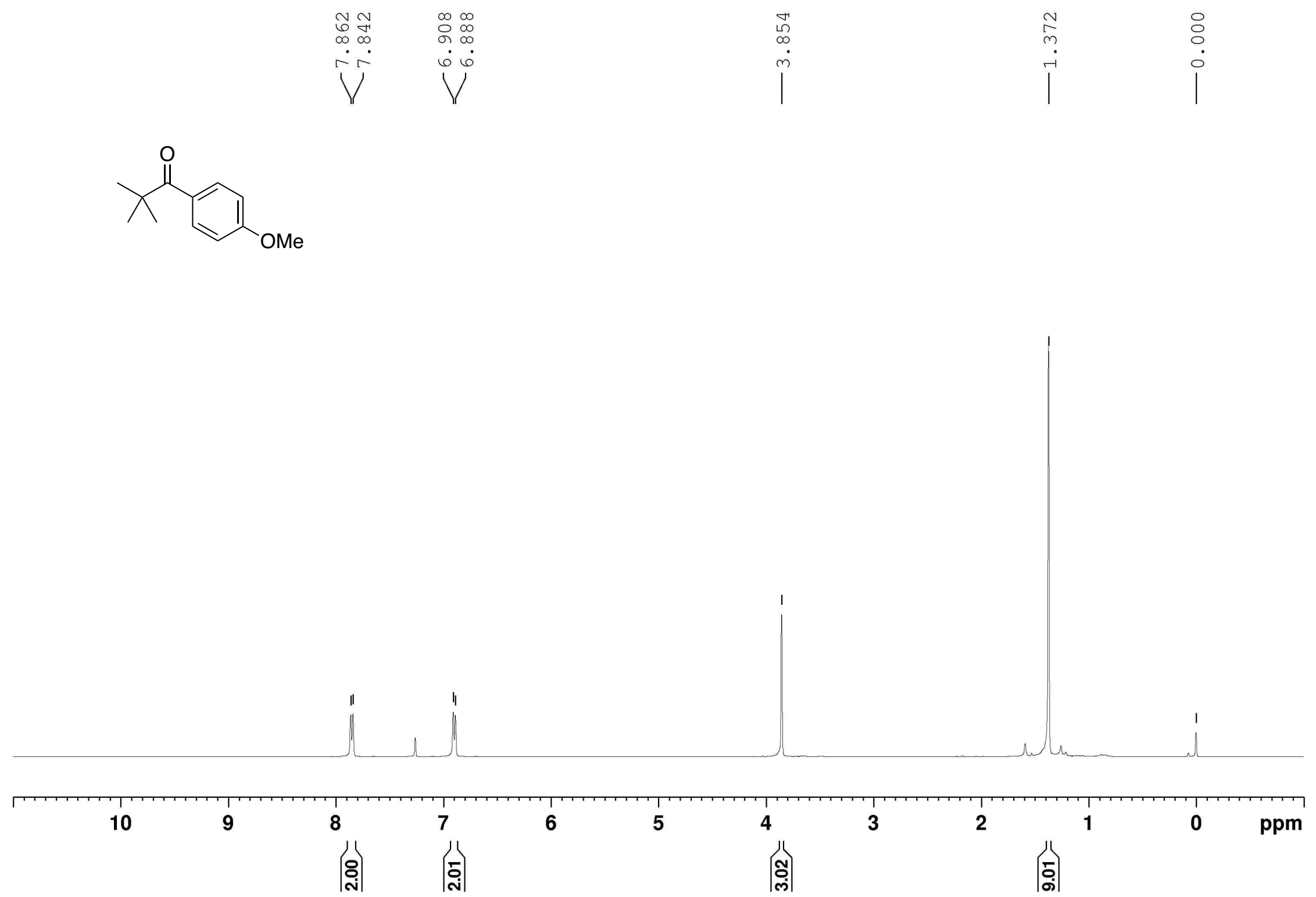
${ }^{13} \mathrm{C}$ NMR of $4 a e$
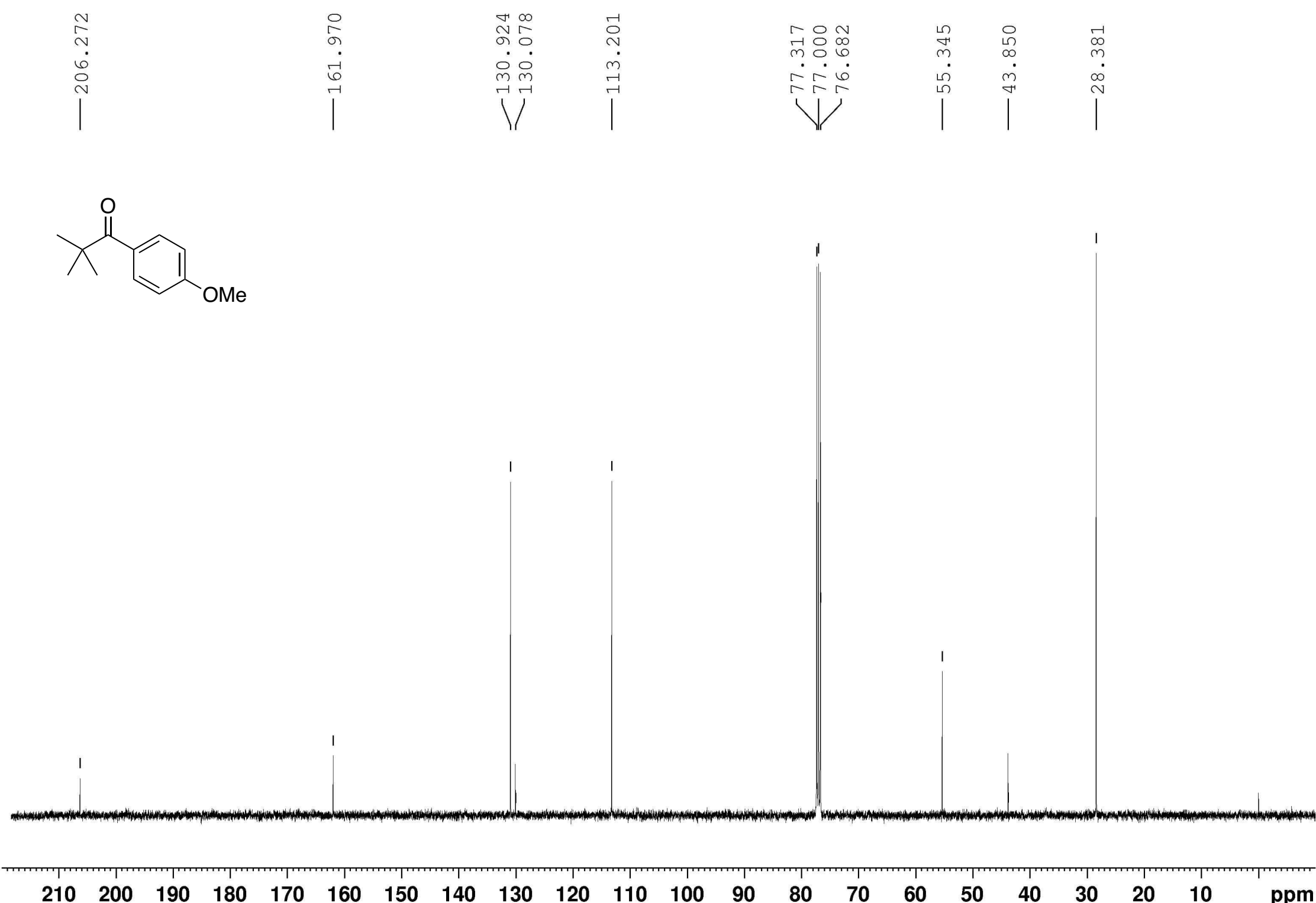

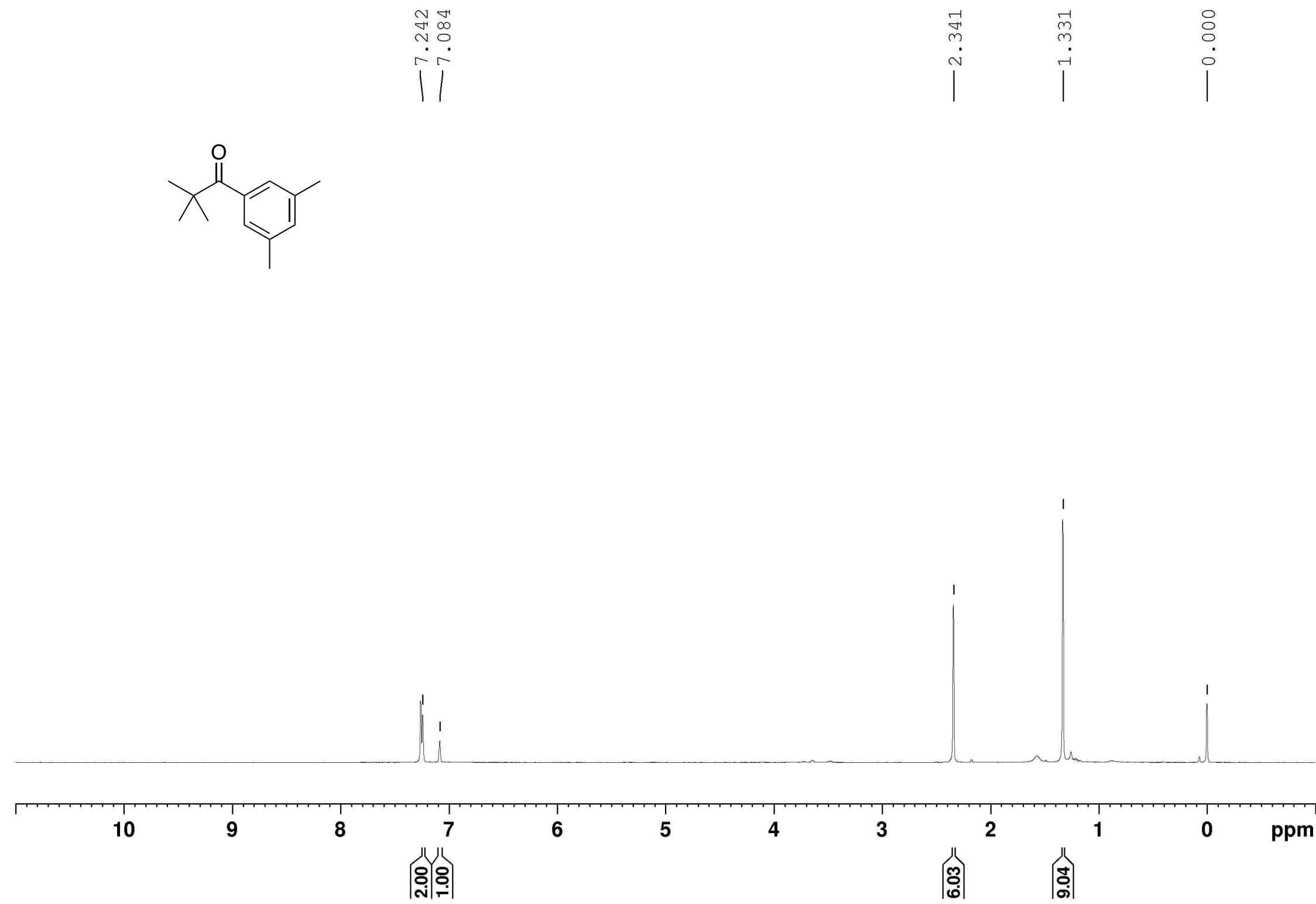
${ }^{13} \mathrm{C}$ NMR of $4 \mathbf{a f}$
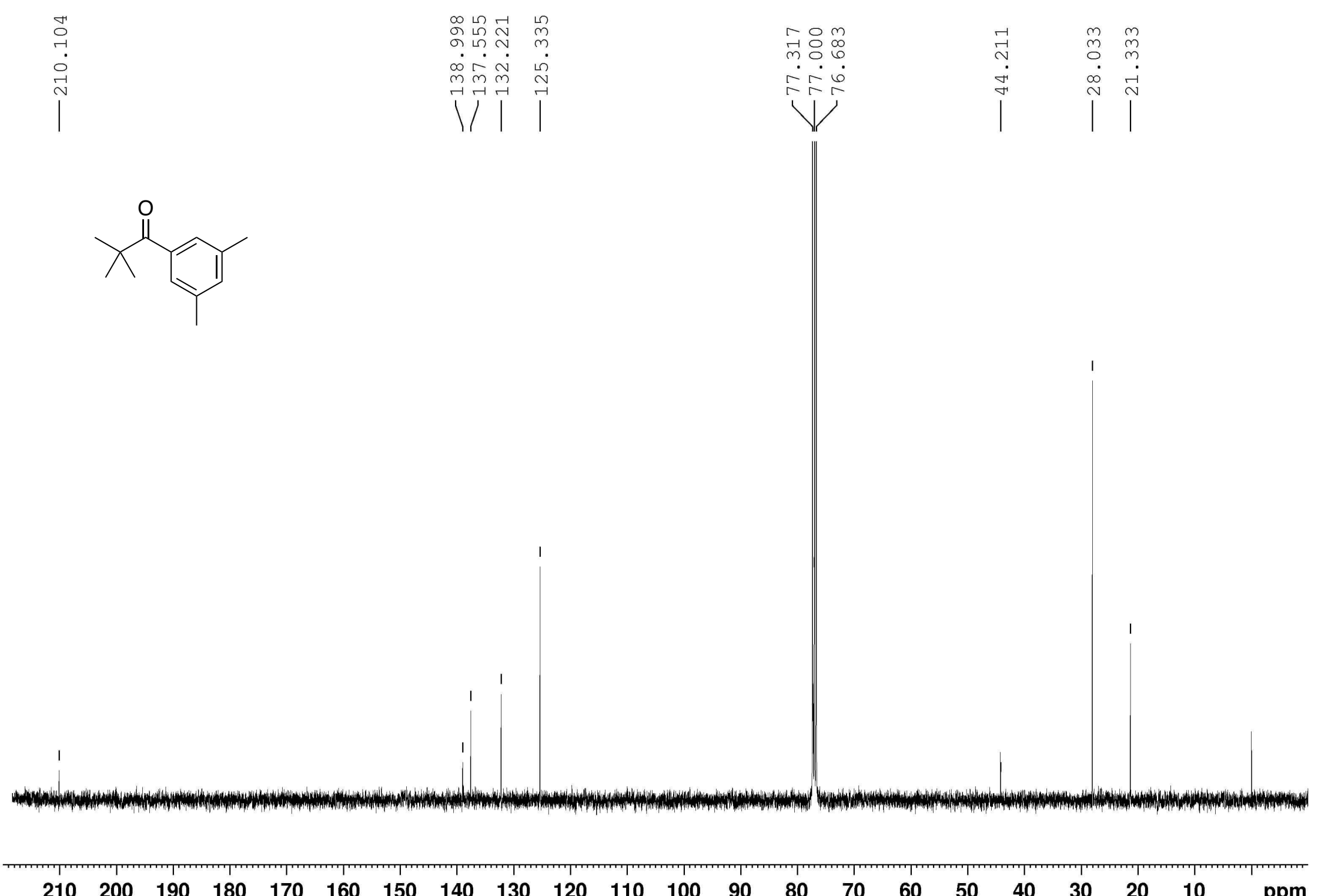

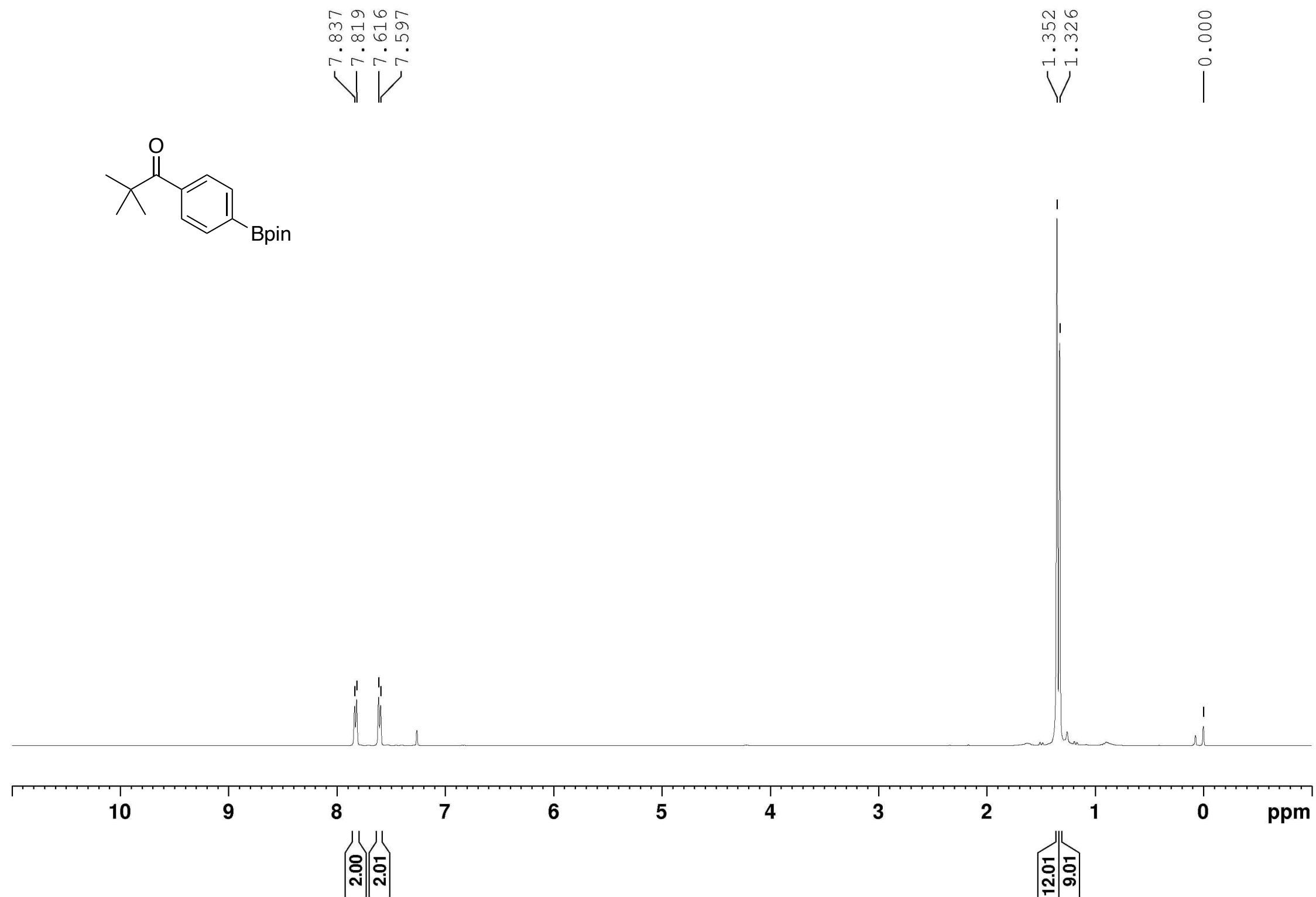
${ }^{13} \mathrm{C}$ NMR of $4 a d$
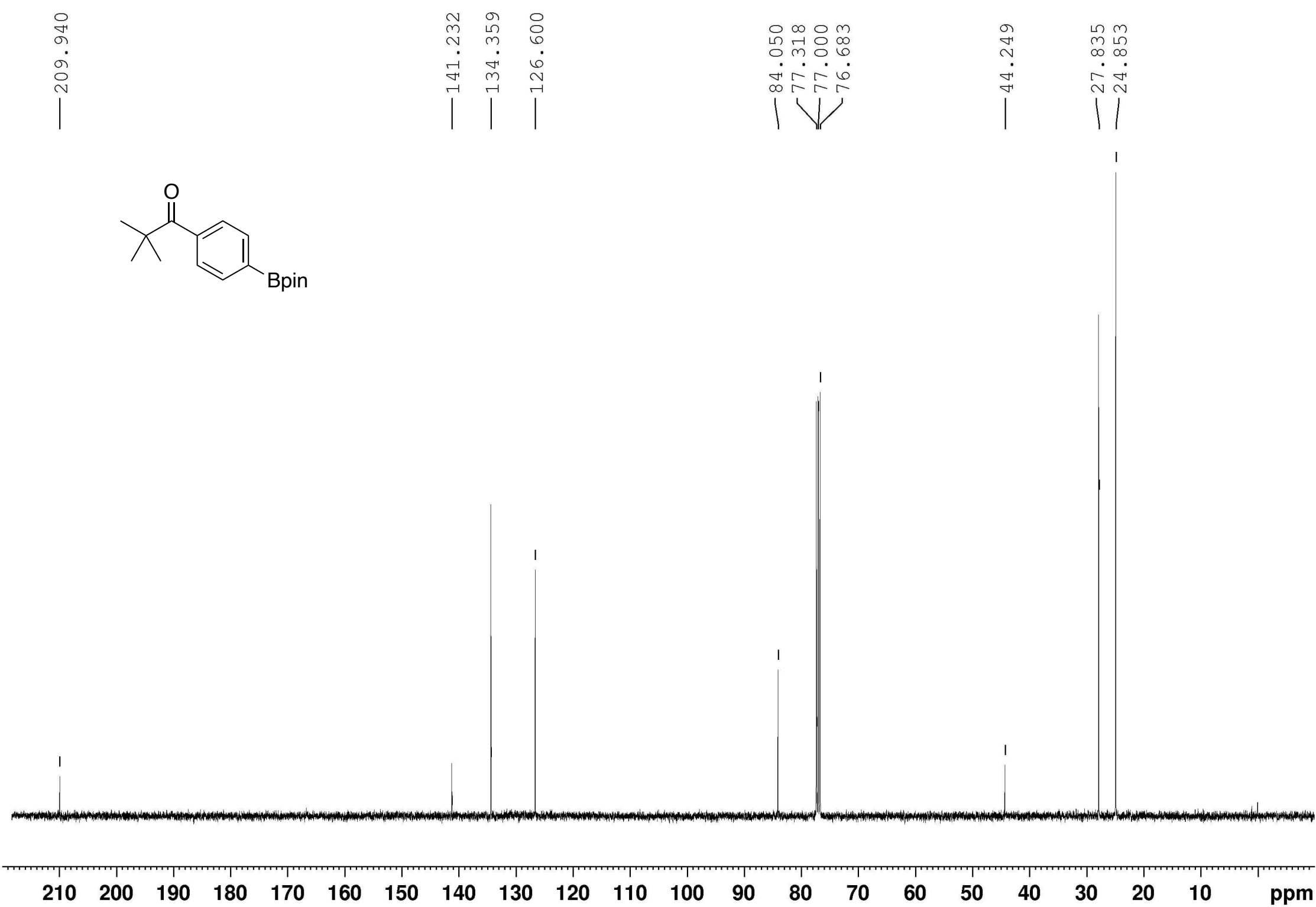
${ }^{11} \mathrm{~B}$ NMR of $4 \mathbf{a d}$
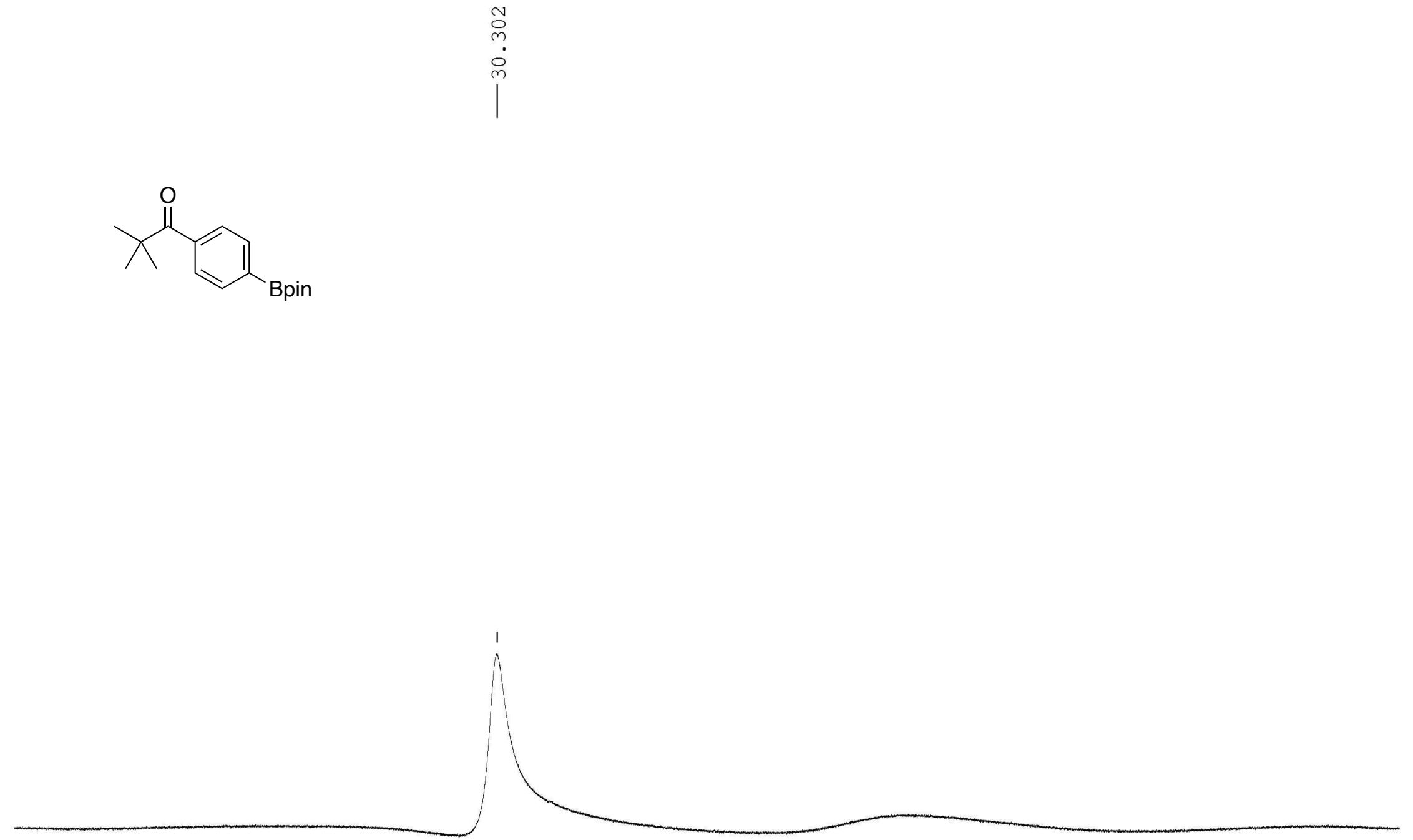


\section{${ }^{1} \mathrm{H}$ NMR of $\mathbf{4 a g}$}
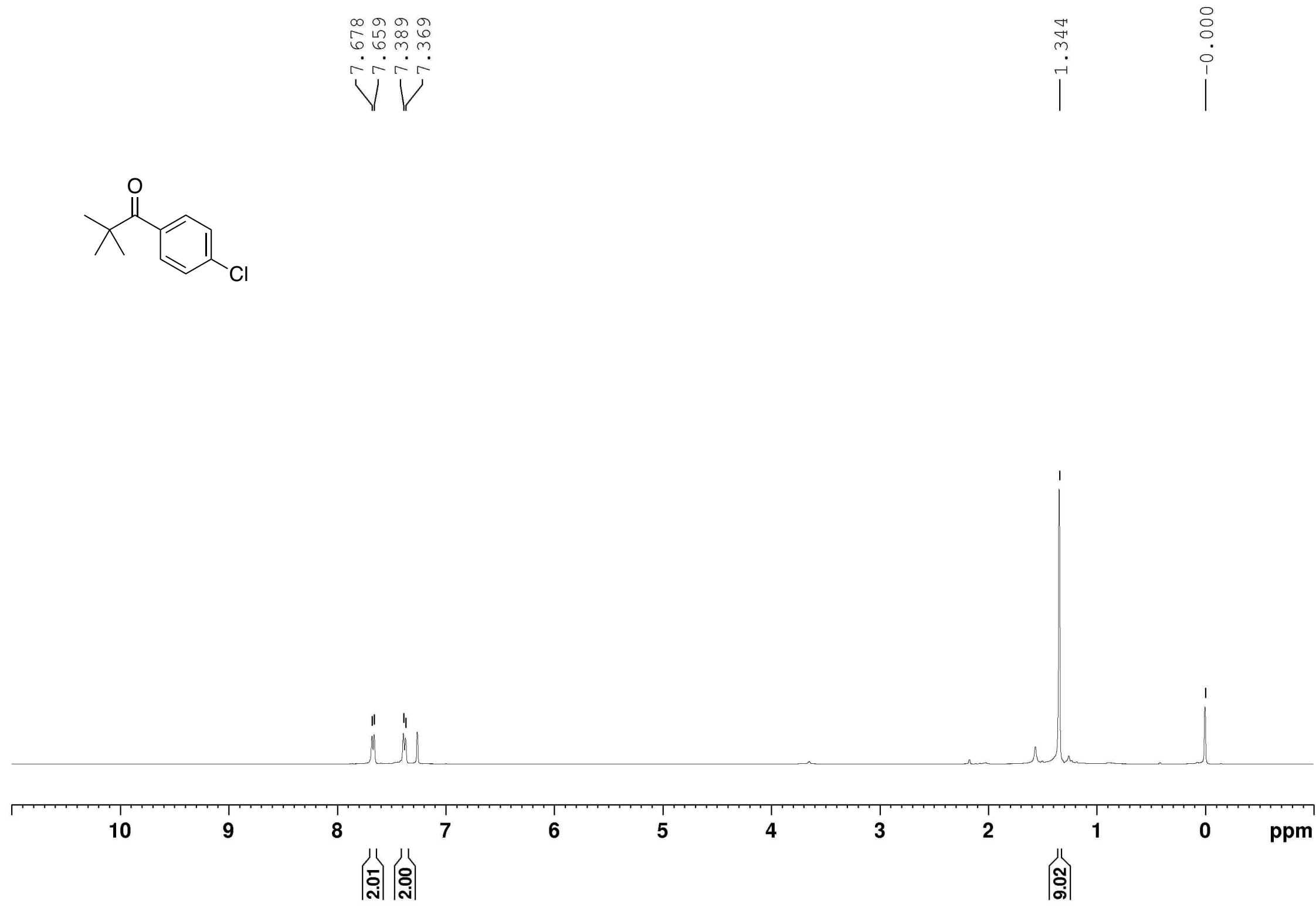
${ }^{13} \mathrm{C}$ NMR of $4 a g$

\begin{tabular}{l}
$\infty$ \\
\multirow{2}{0}{} \\
$\stackrel{0}{0}$ \\
$\stackrel{0}{0}$ \\
1
\end{tabular}
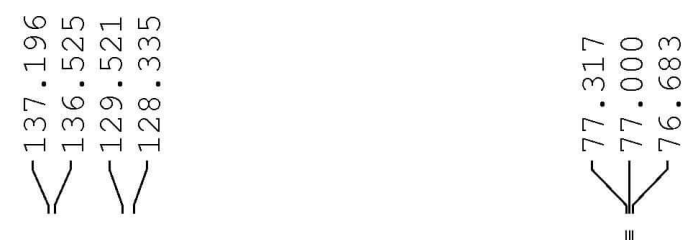

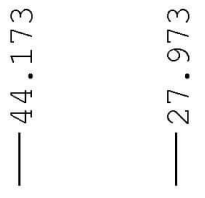
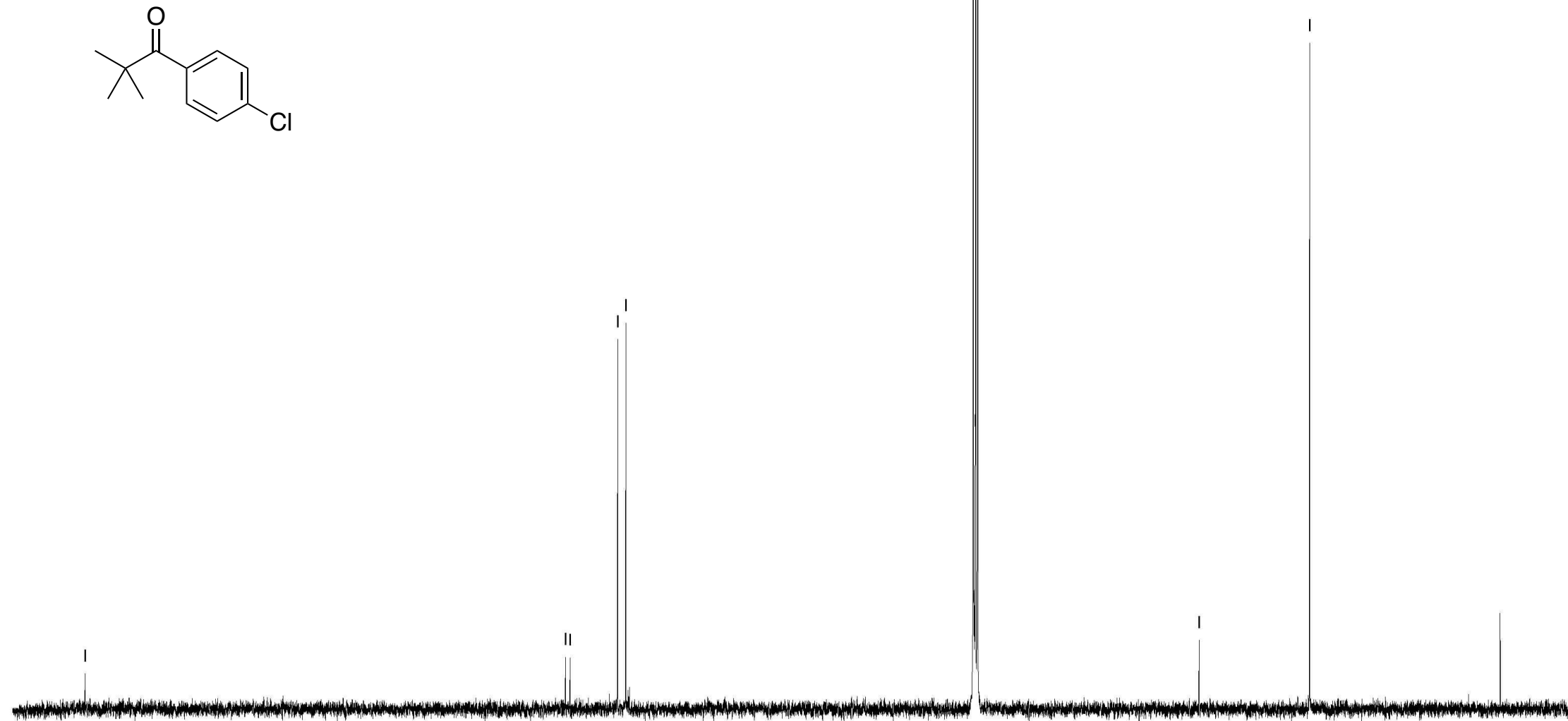

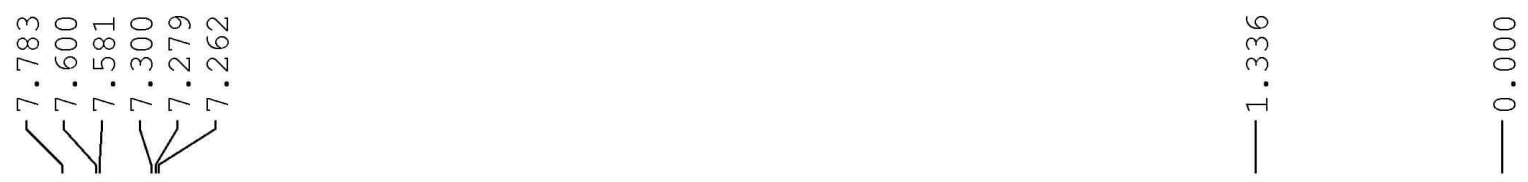<smiles>CC(C)(C)C(=O)c1cccc(Br)c1</smiles>

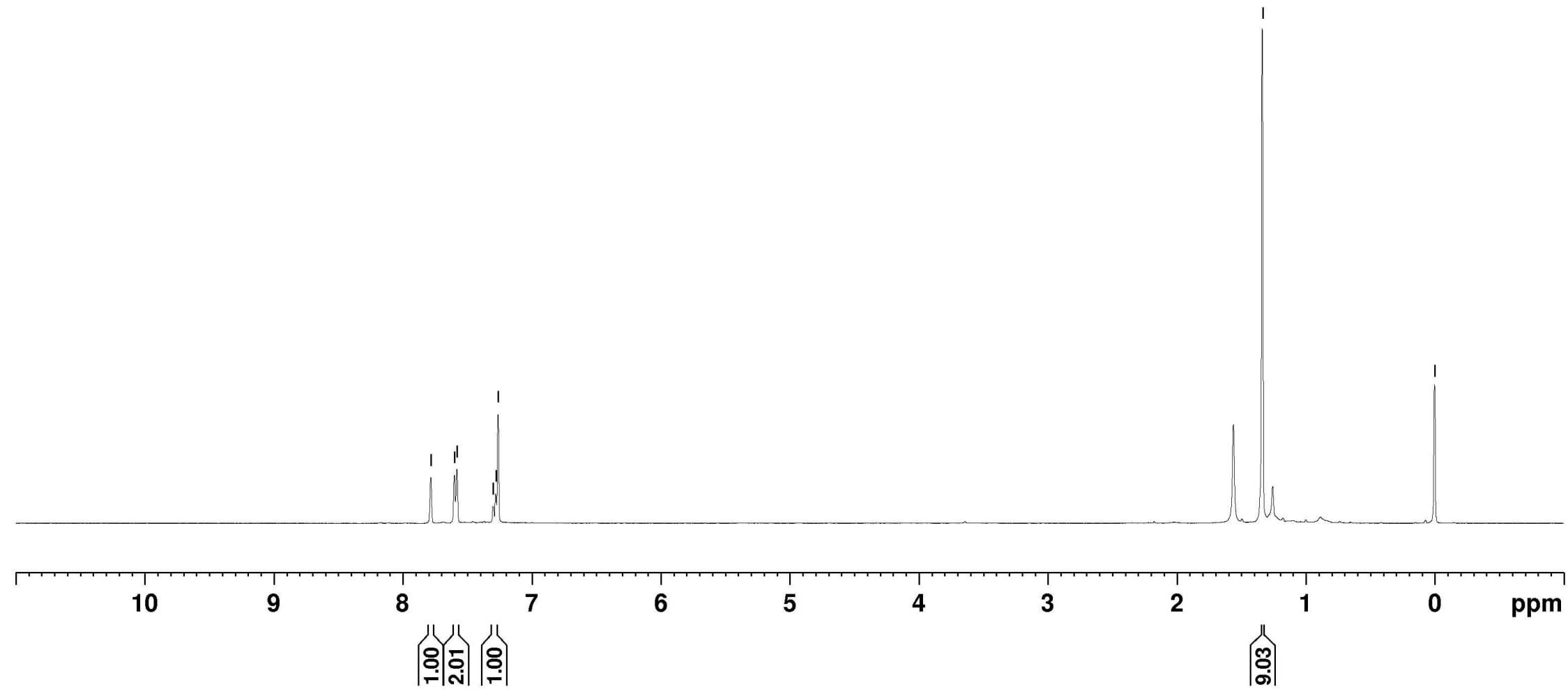


${ }^{13} \mathrm{C}$ NMR of $\mathbf{4 a h}$

\begin{tabular}{l} 
ने \\
$\infty$ \\
$\vdots$ \\
$\circ$ \\
\multirow{2}{1}{} \\
1
\end{tabular}
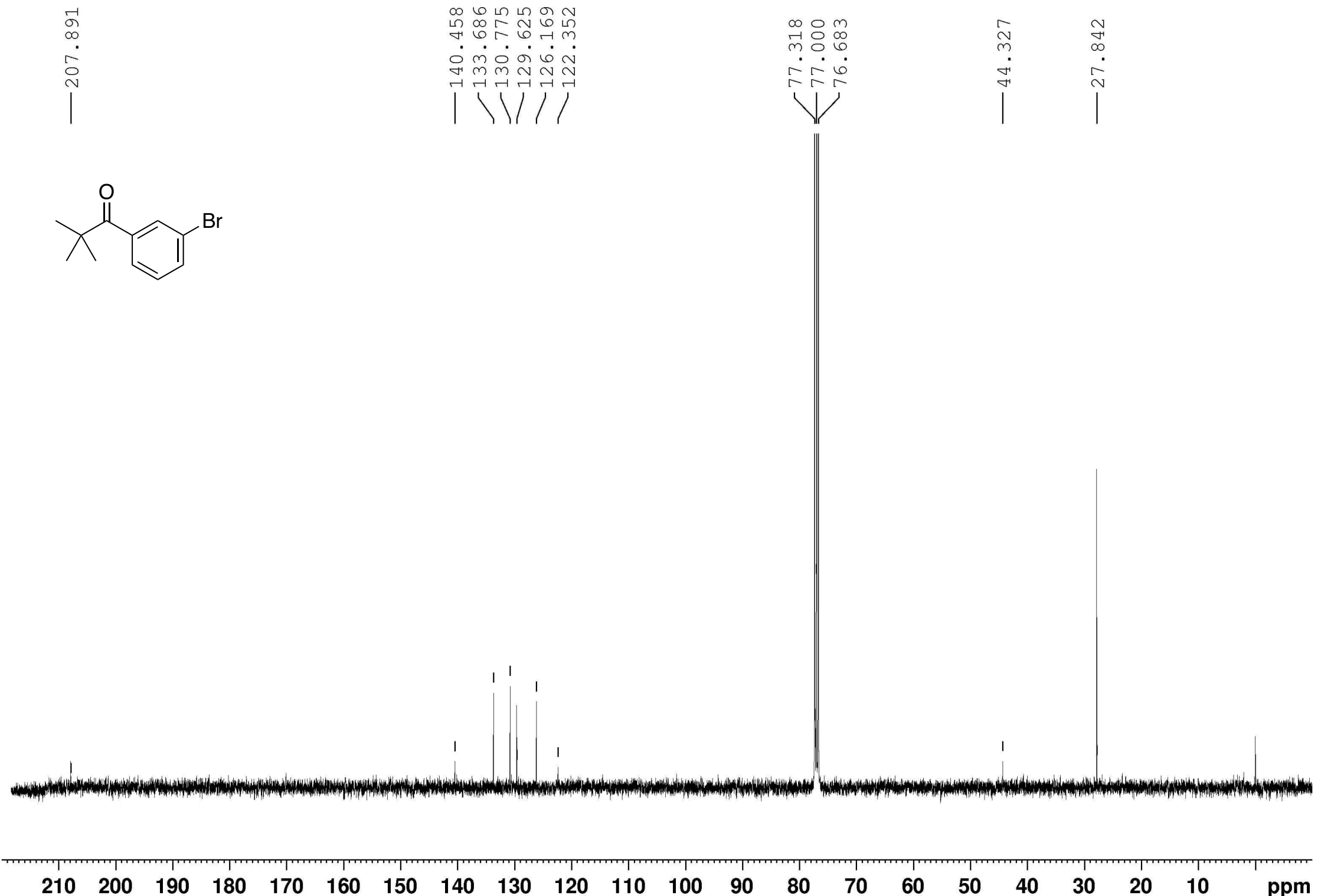
${ }^{1} \mathrm{H}$ NMR of 4ai
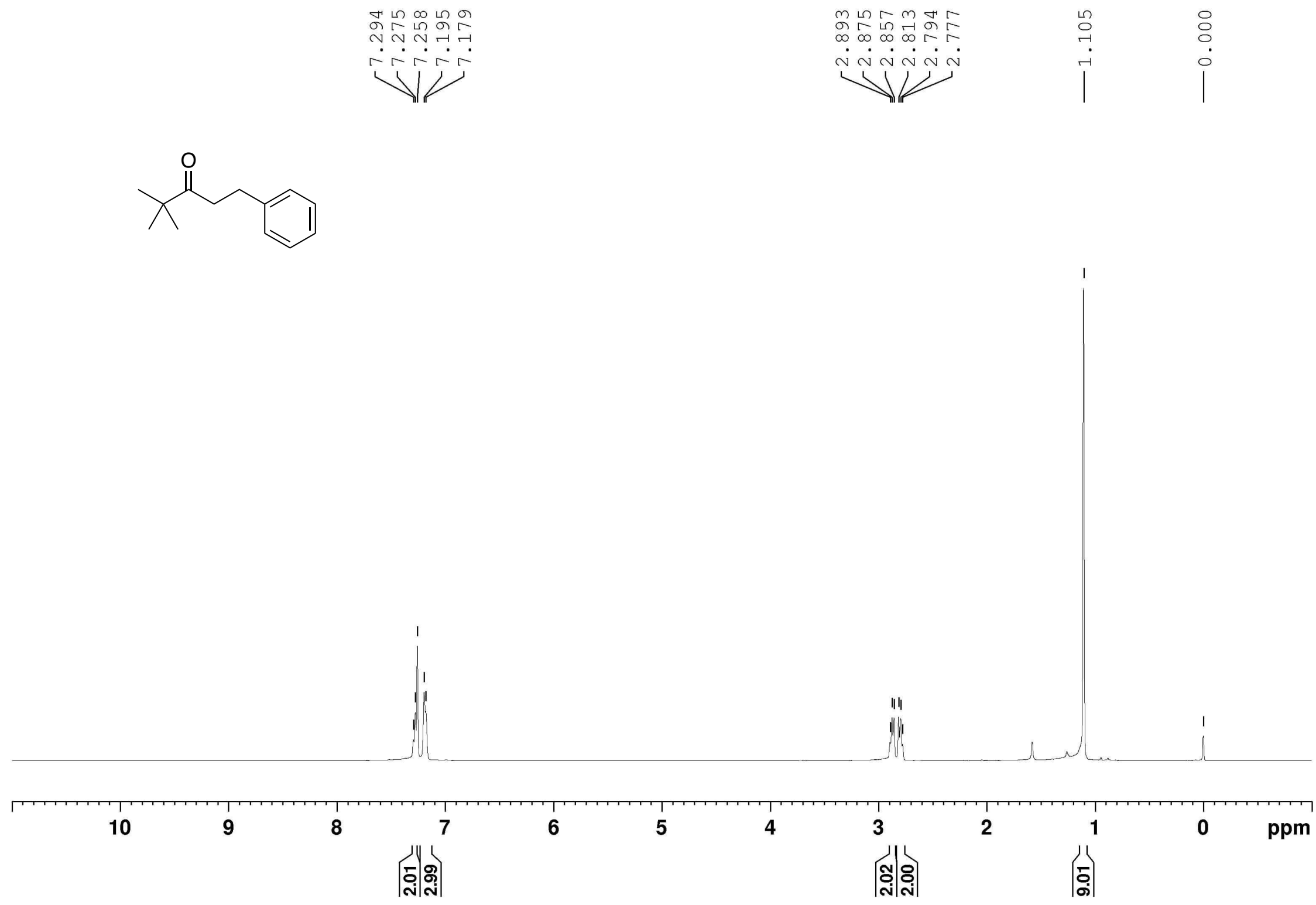
${ }^{13} \mathrm{C}$ NMR of 4ai
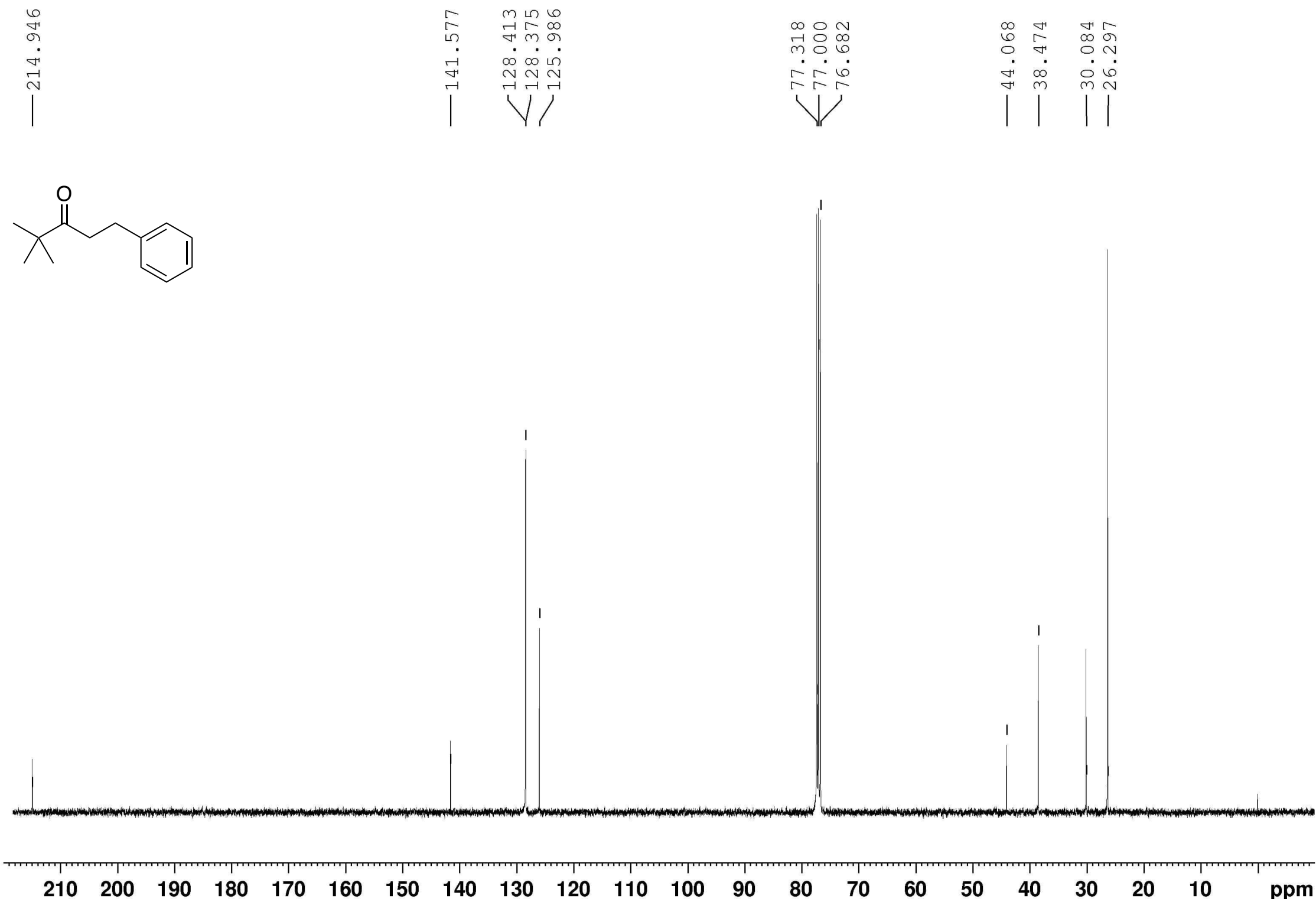

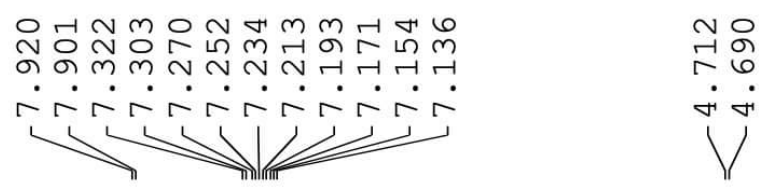

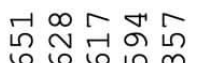
जं लंखं nNN نं

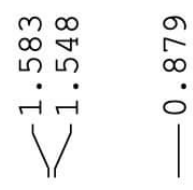

$\circ$
0
0
1
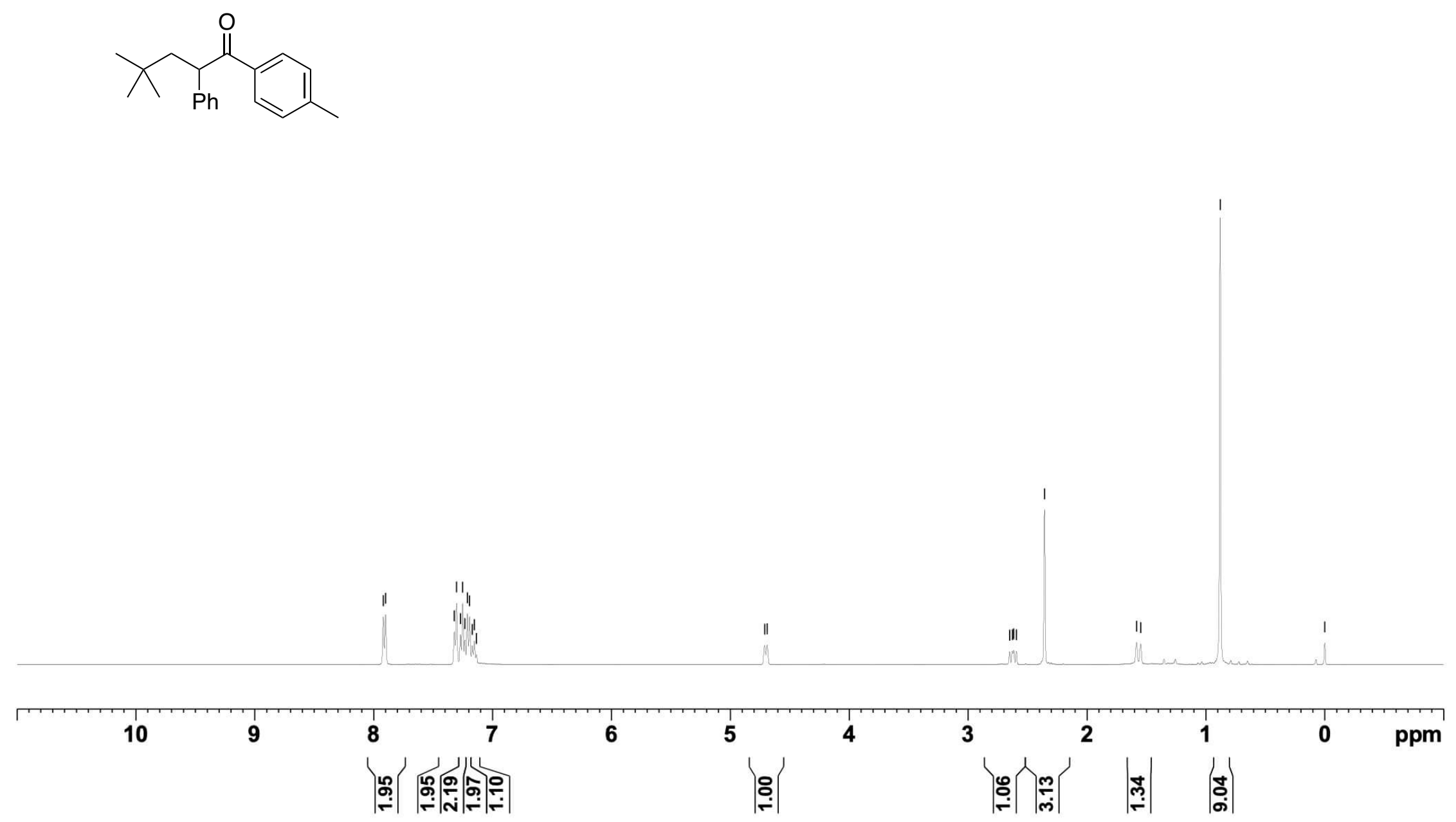


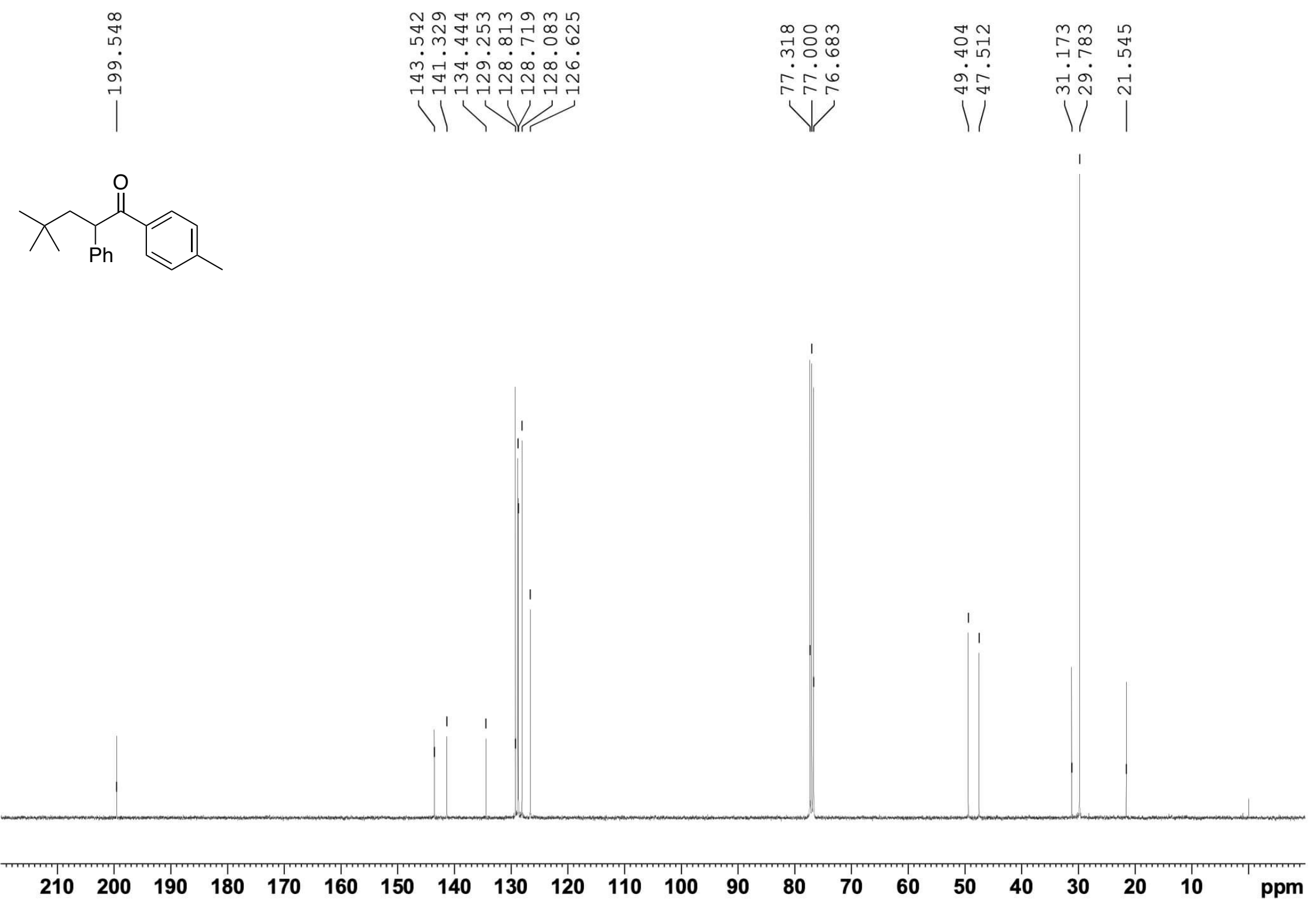



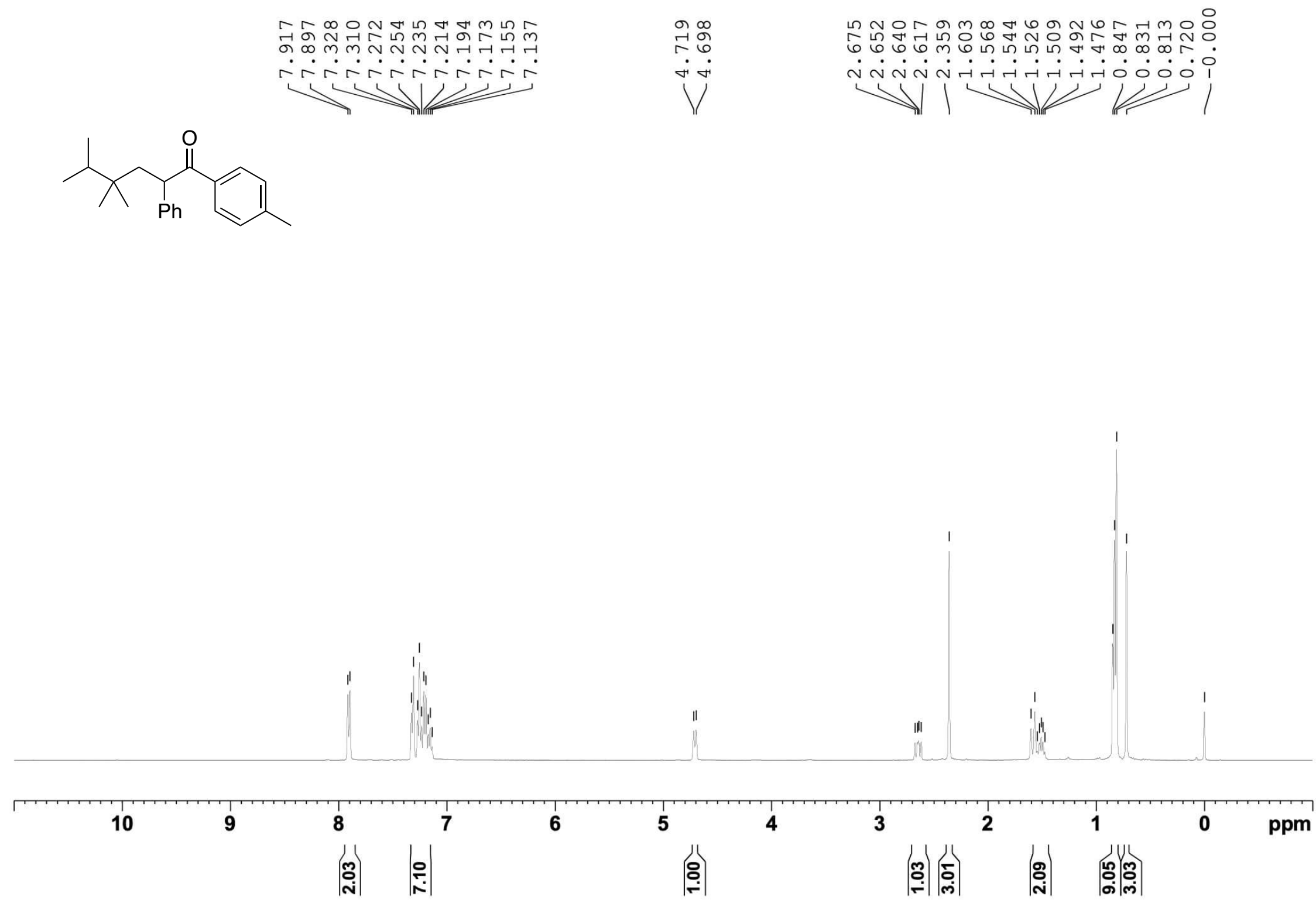

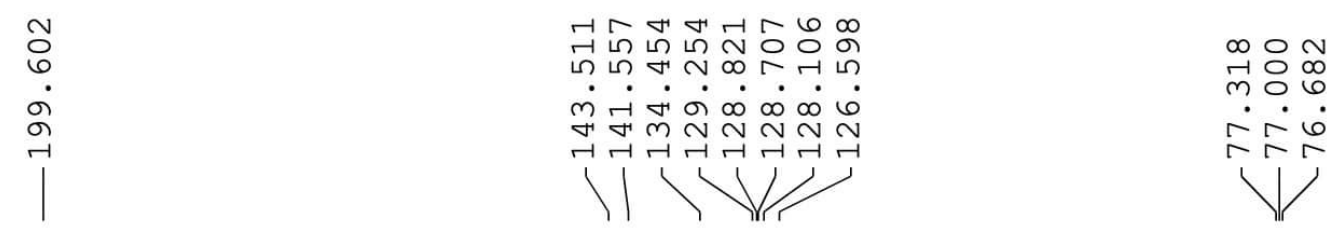

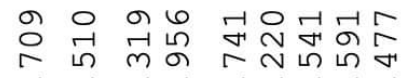

ஆ लூ ம்

।V VIV
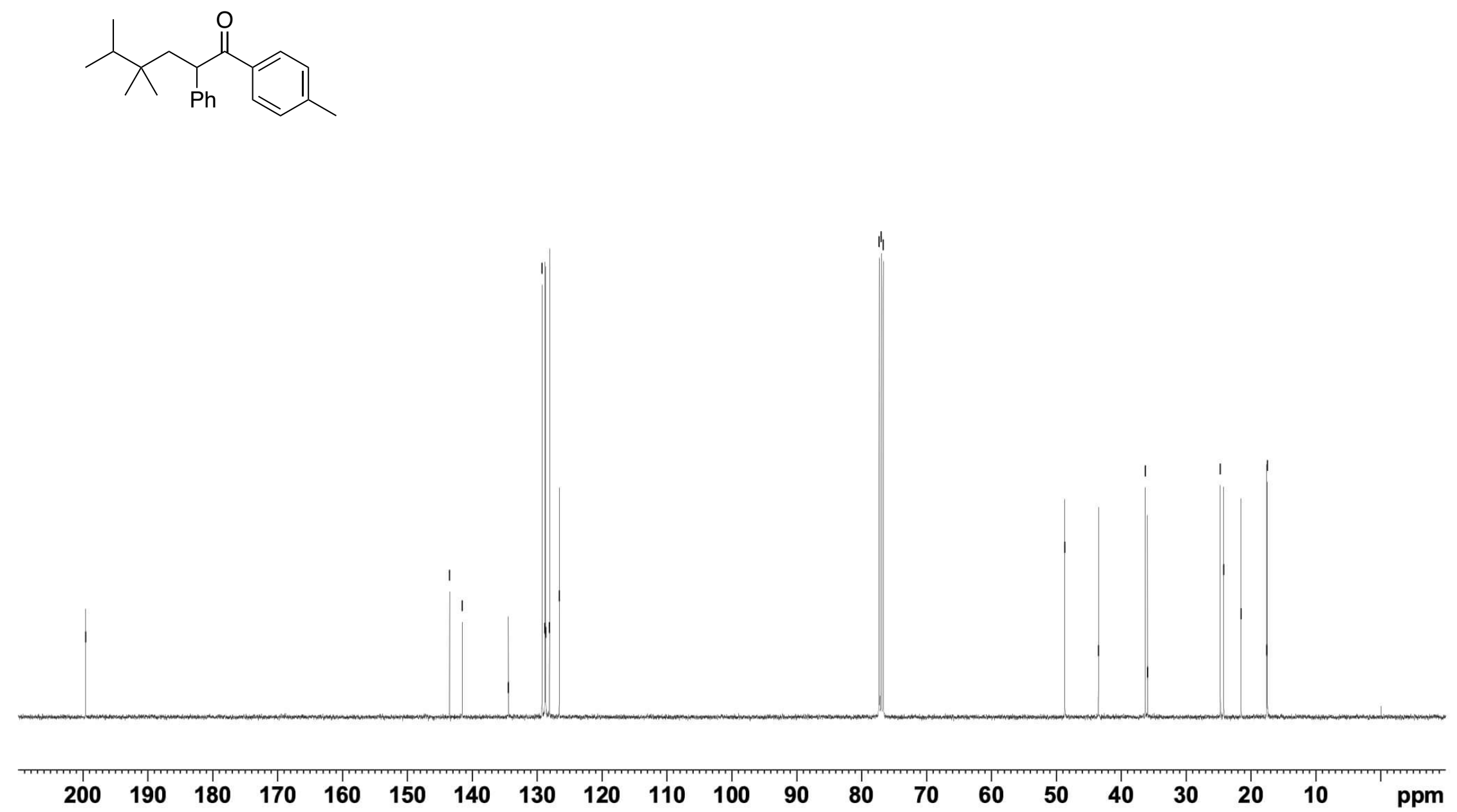

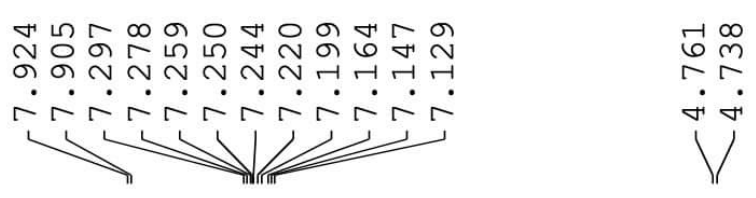

Ұ유궁

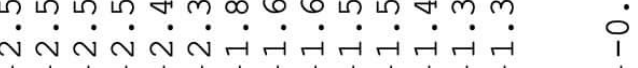
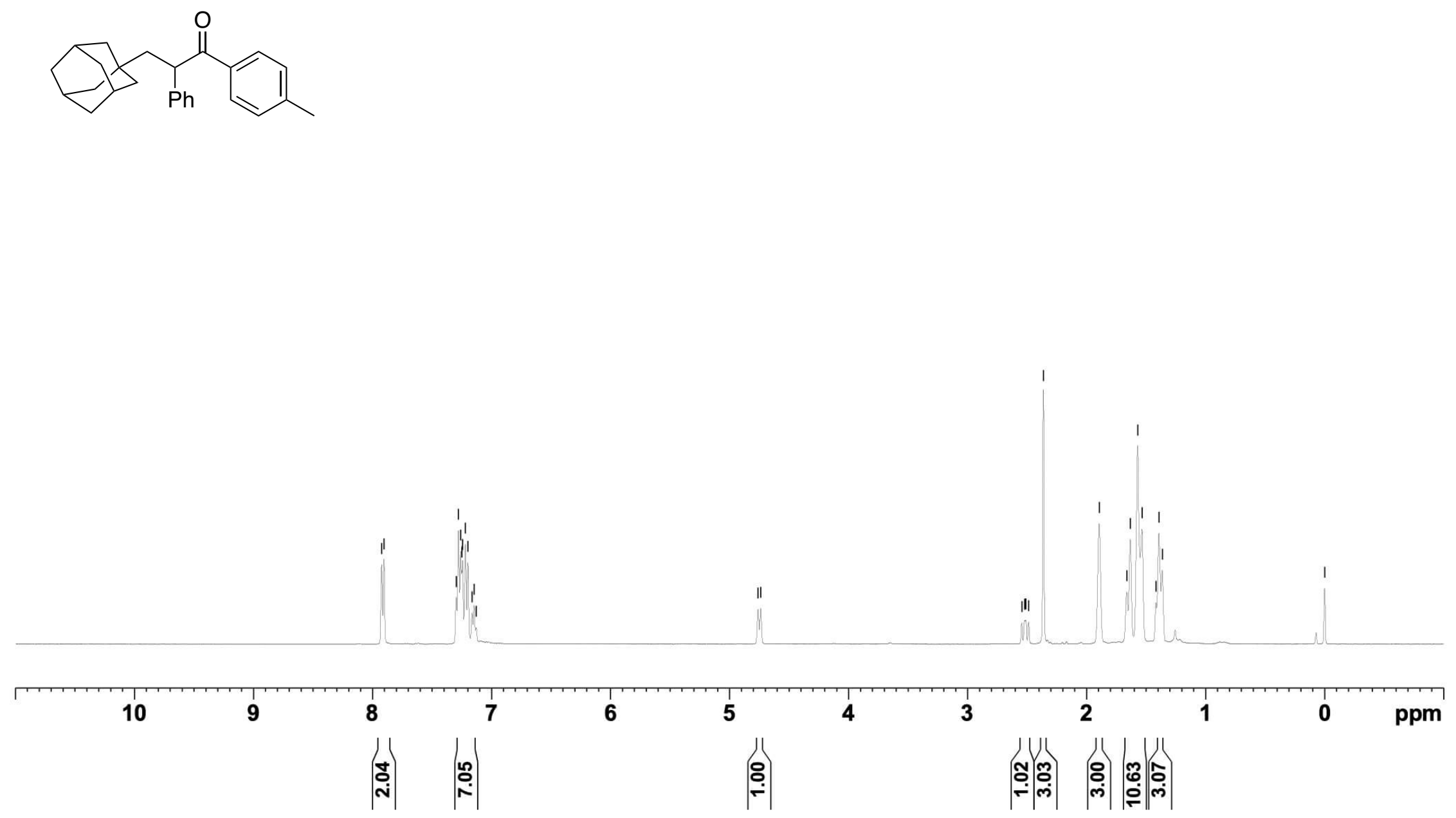


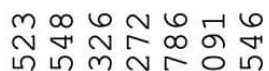

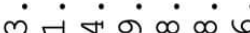

承

1)

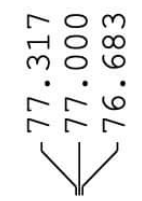

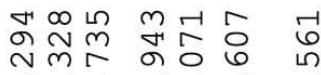

$\dot{\infty} \dot{\sim} \dot{\varphi} \dot{m} \dot{\infty} \dot{\imath}$

ll | |
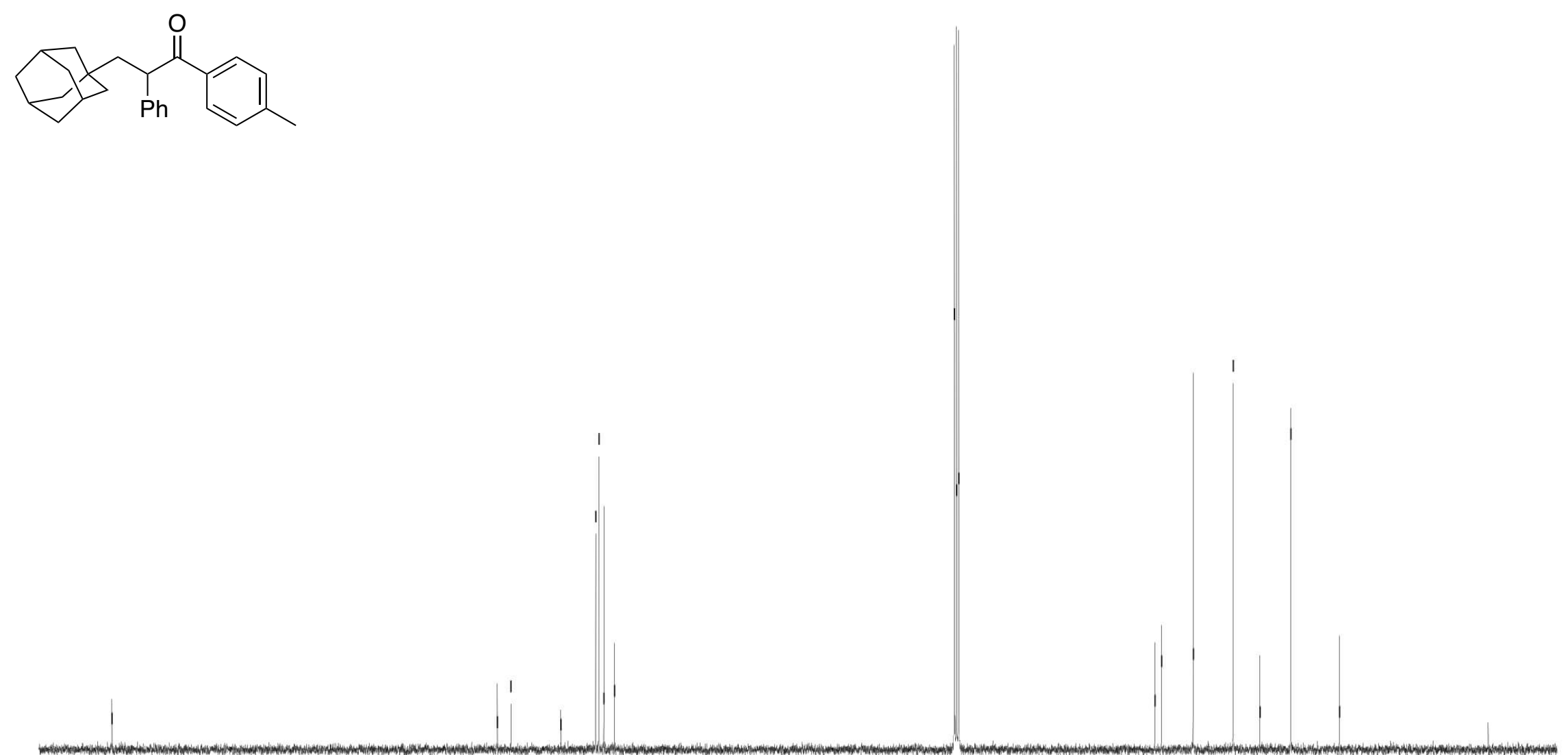

$\begin{array}{lllllllllllllllllllll}200 & 190 & 180 & 170 & 160 & 150 & 140 & 130 & 120 & 110 & 100 & 90 & 80 & 70 & 60 & 50 & 40 & 30 & 20 & 10 & \mathrm{ppm}\end{array}$ 


\section{${ }^{1} \mathrm{H}$ NMR of $\mathbf{6 i a a}$}

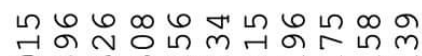

birinisinar

13

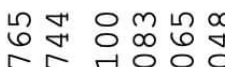

मं मंनंम

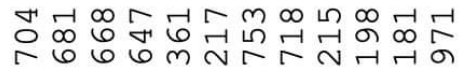

$\dot{n} \dot{n} \dot{\sim} \dot{\sim}$
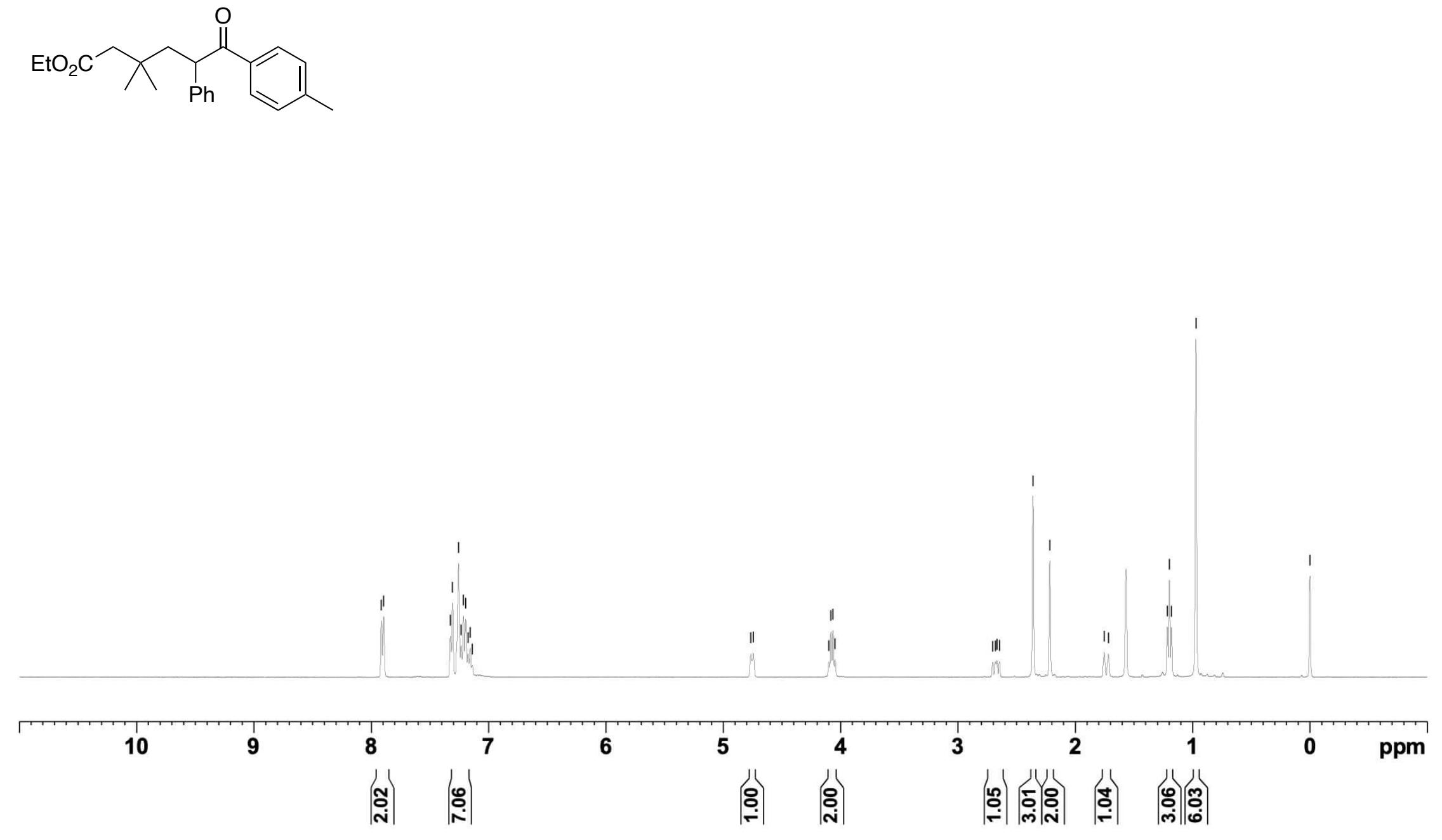

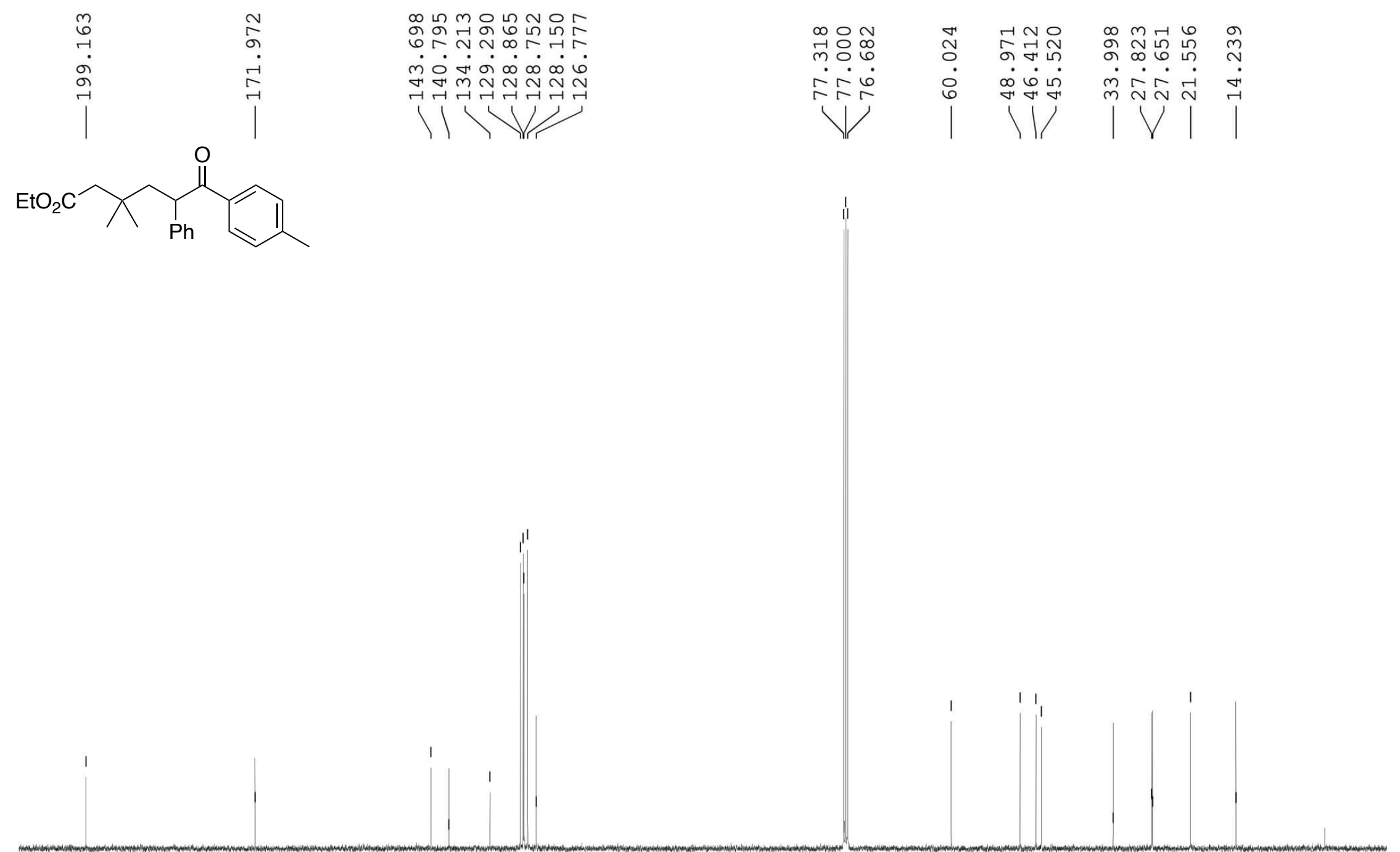

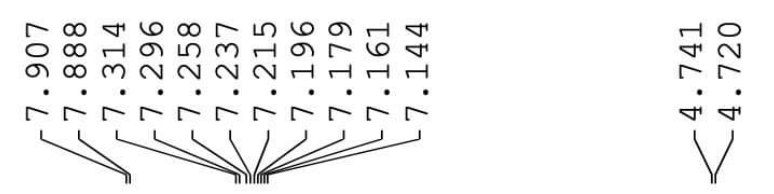

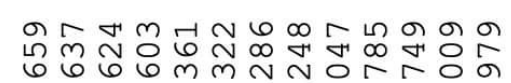

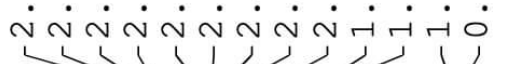

$\circ$
0
0
1
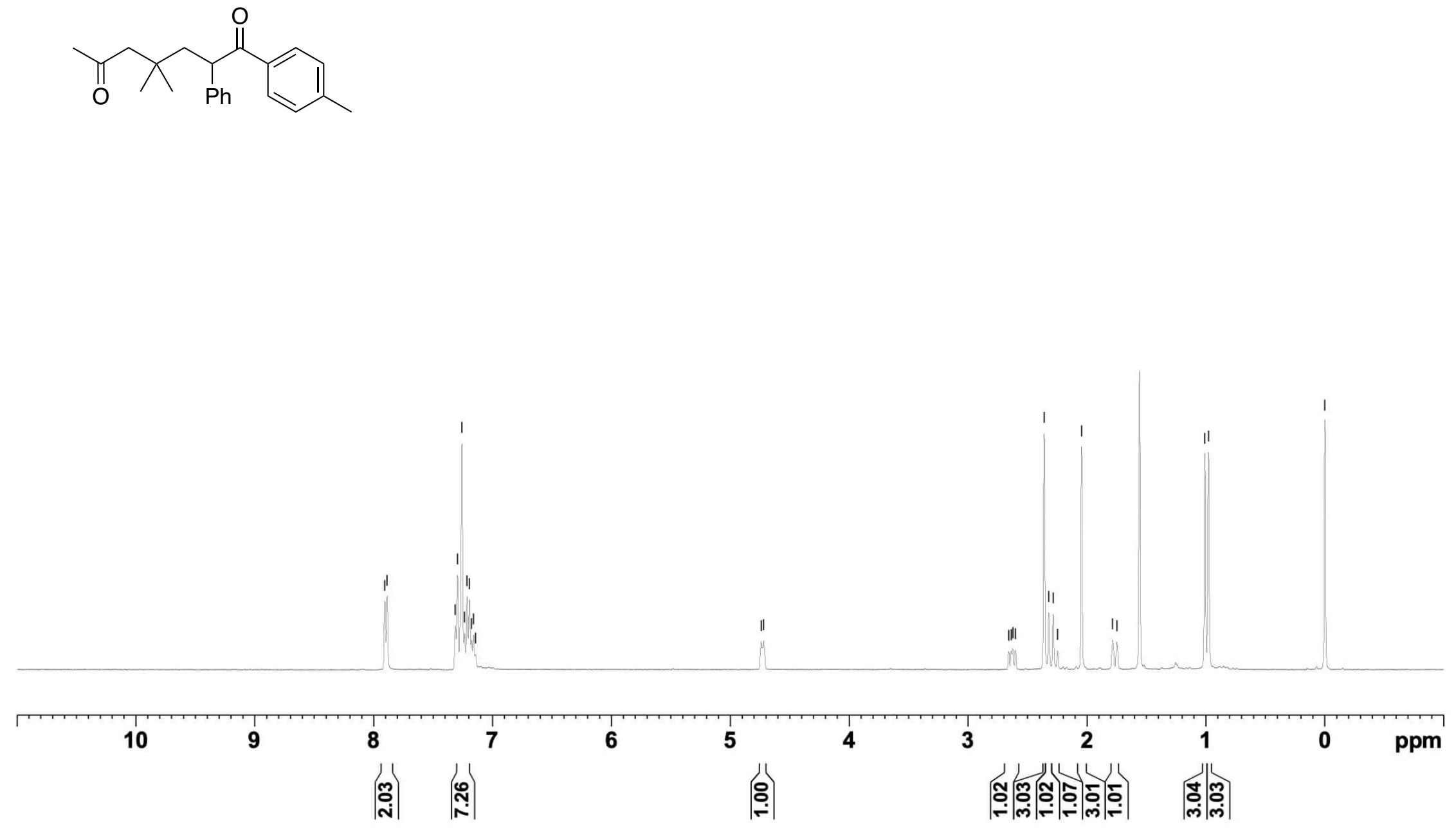

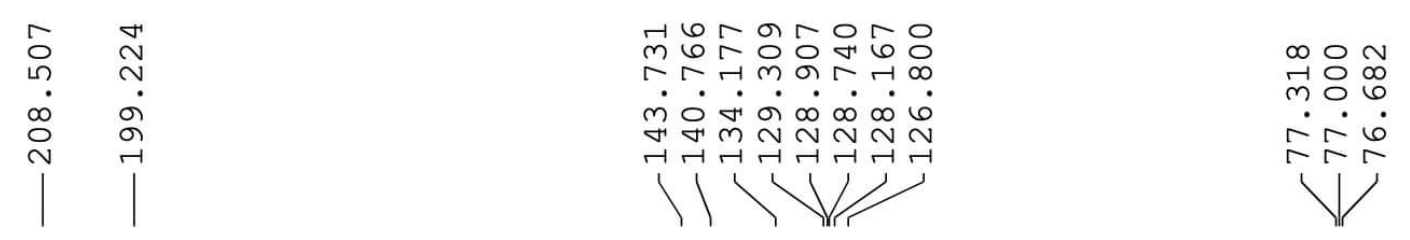

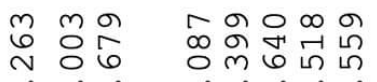

宊

11111
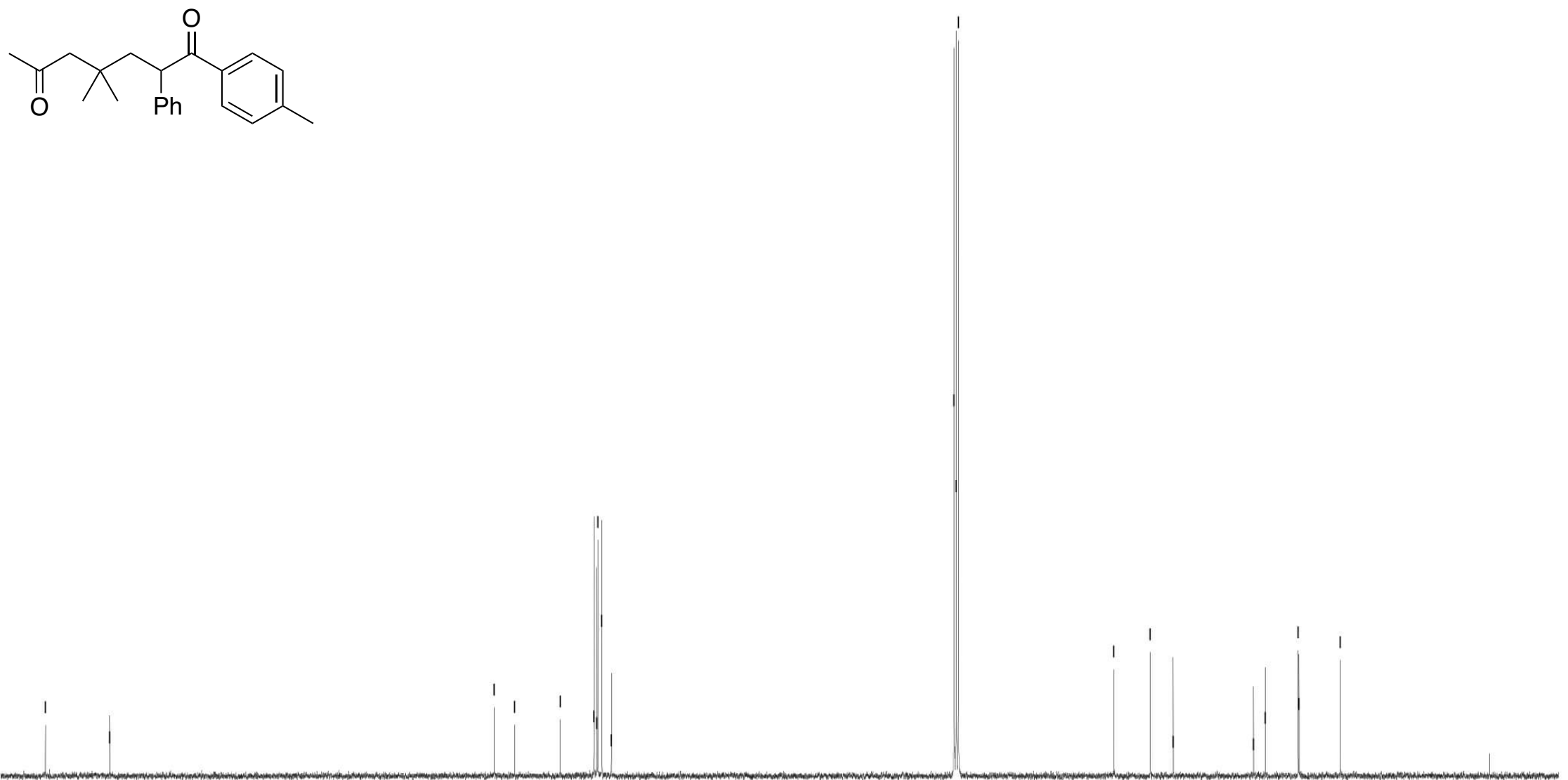

$\begin{array}{lllllllll}70 & 60 & 50 & 40 & 30 & 20 & 10 & \text { ppm }\end{array}$ 


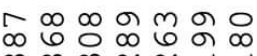

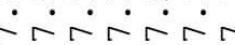

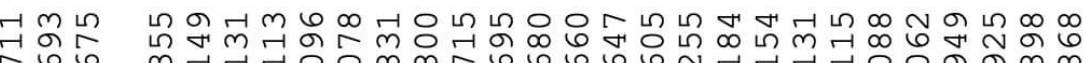

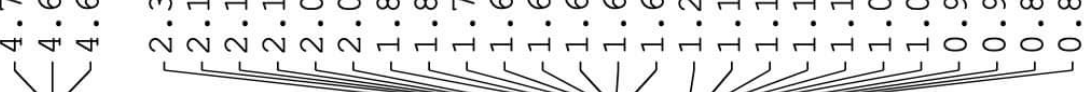
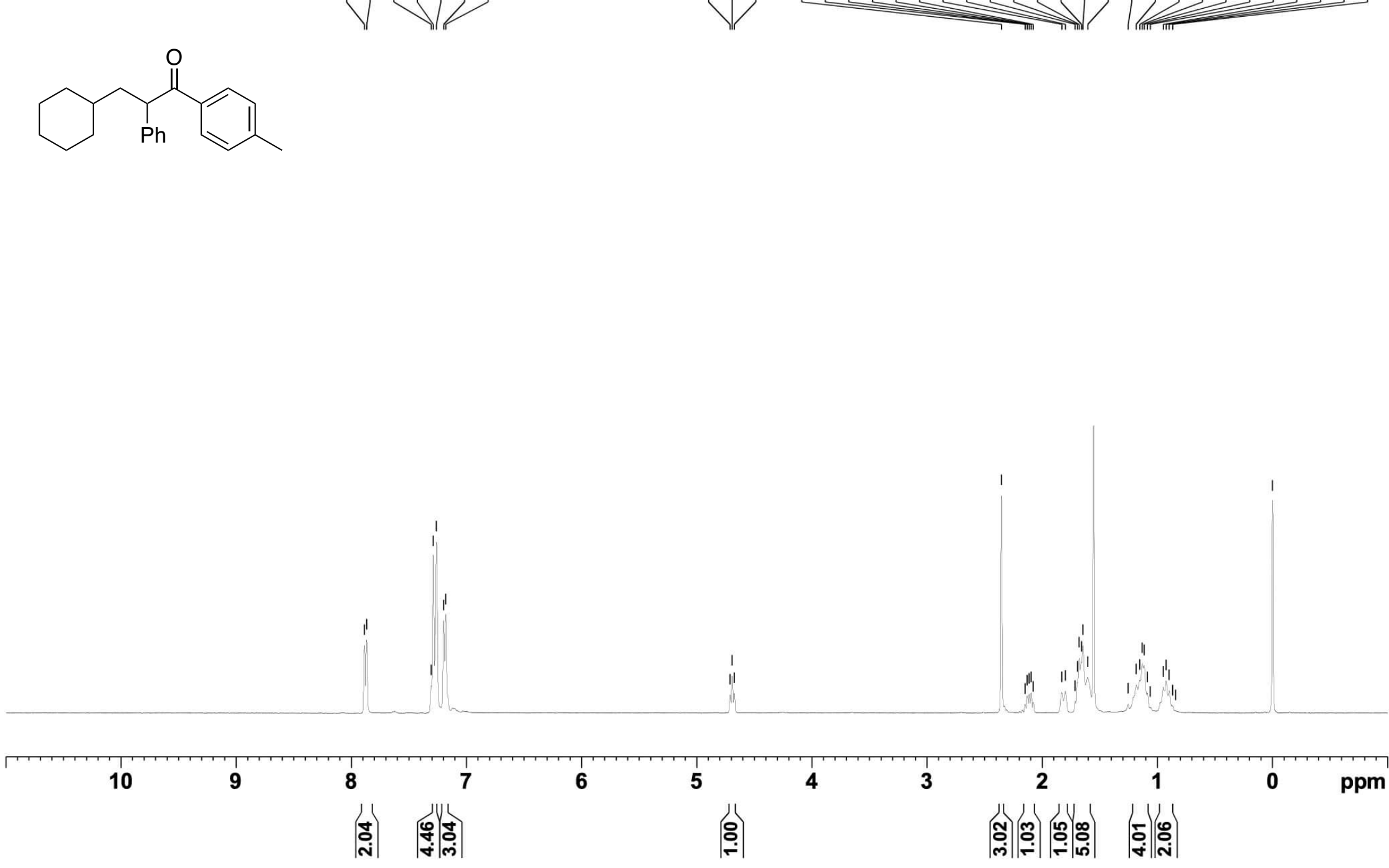


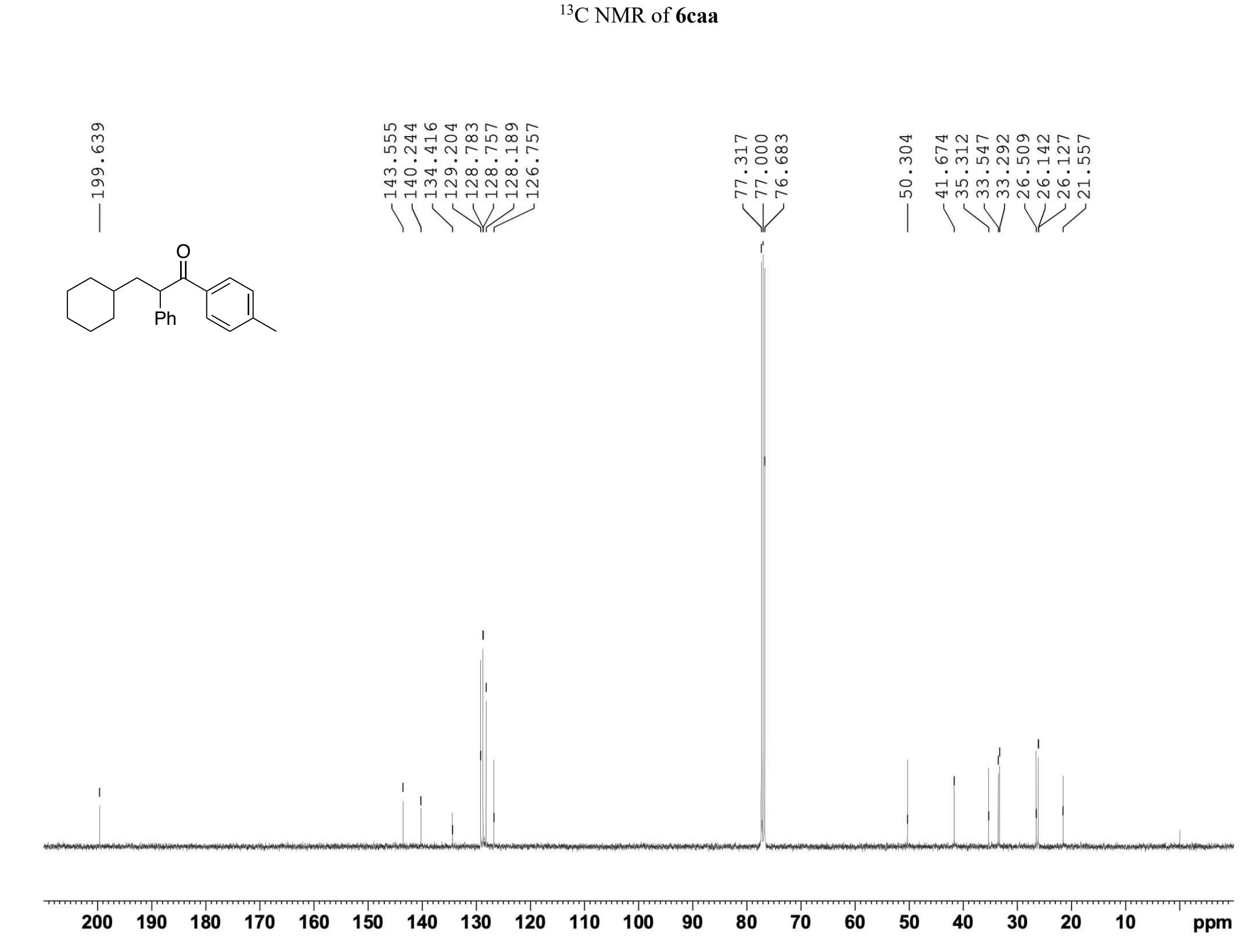




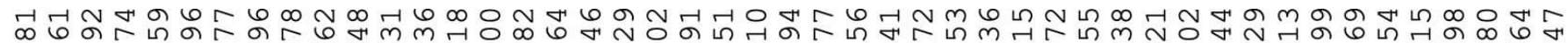

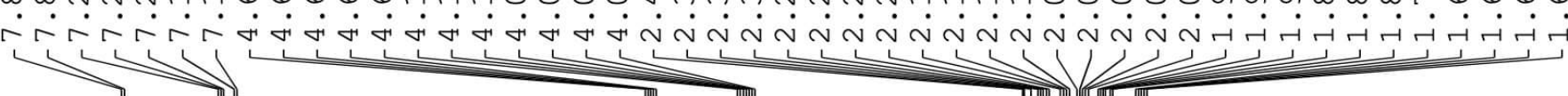
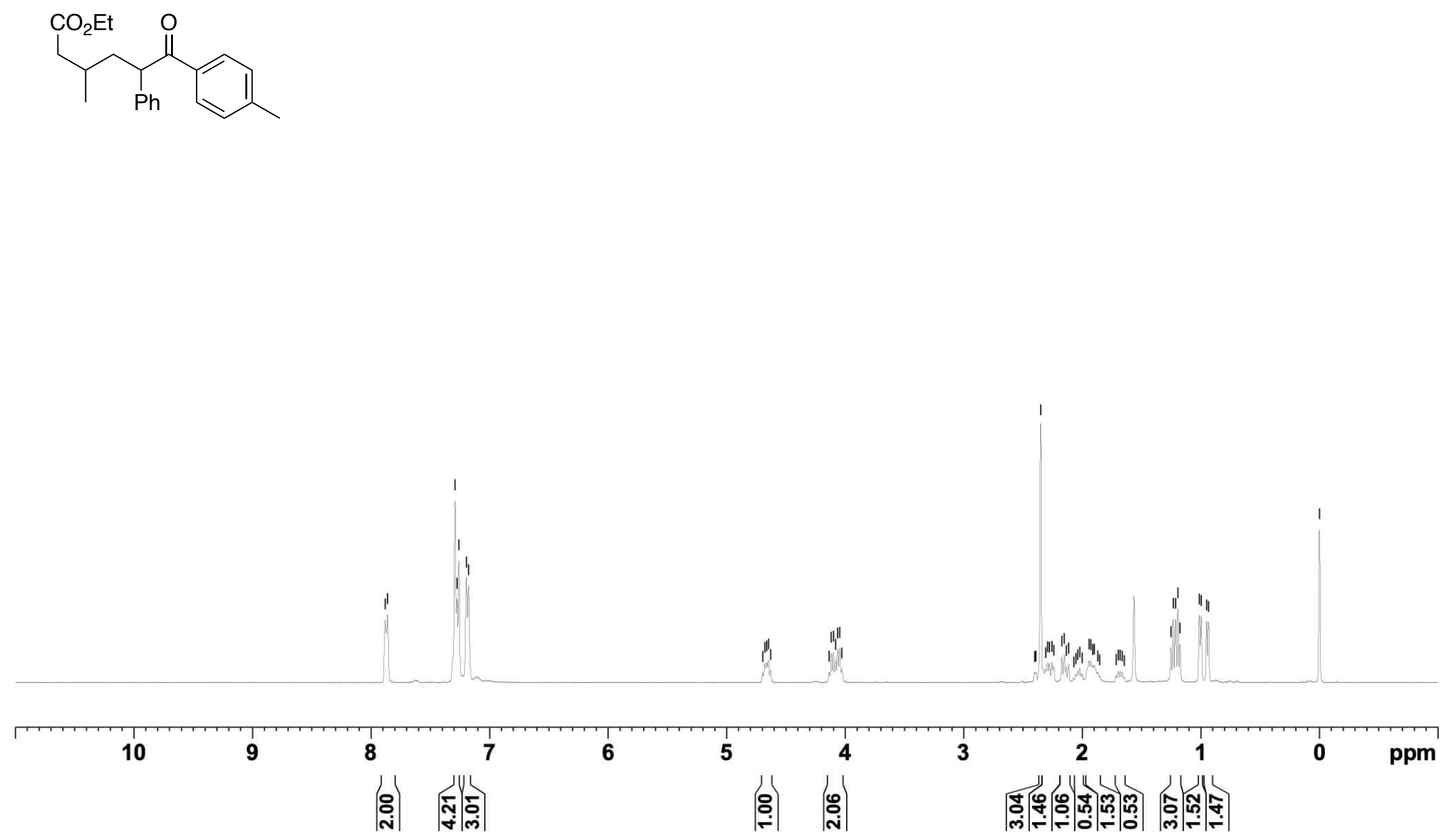

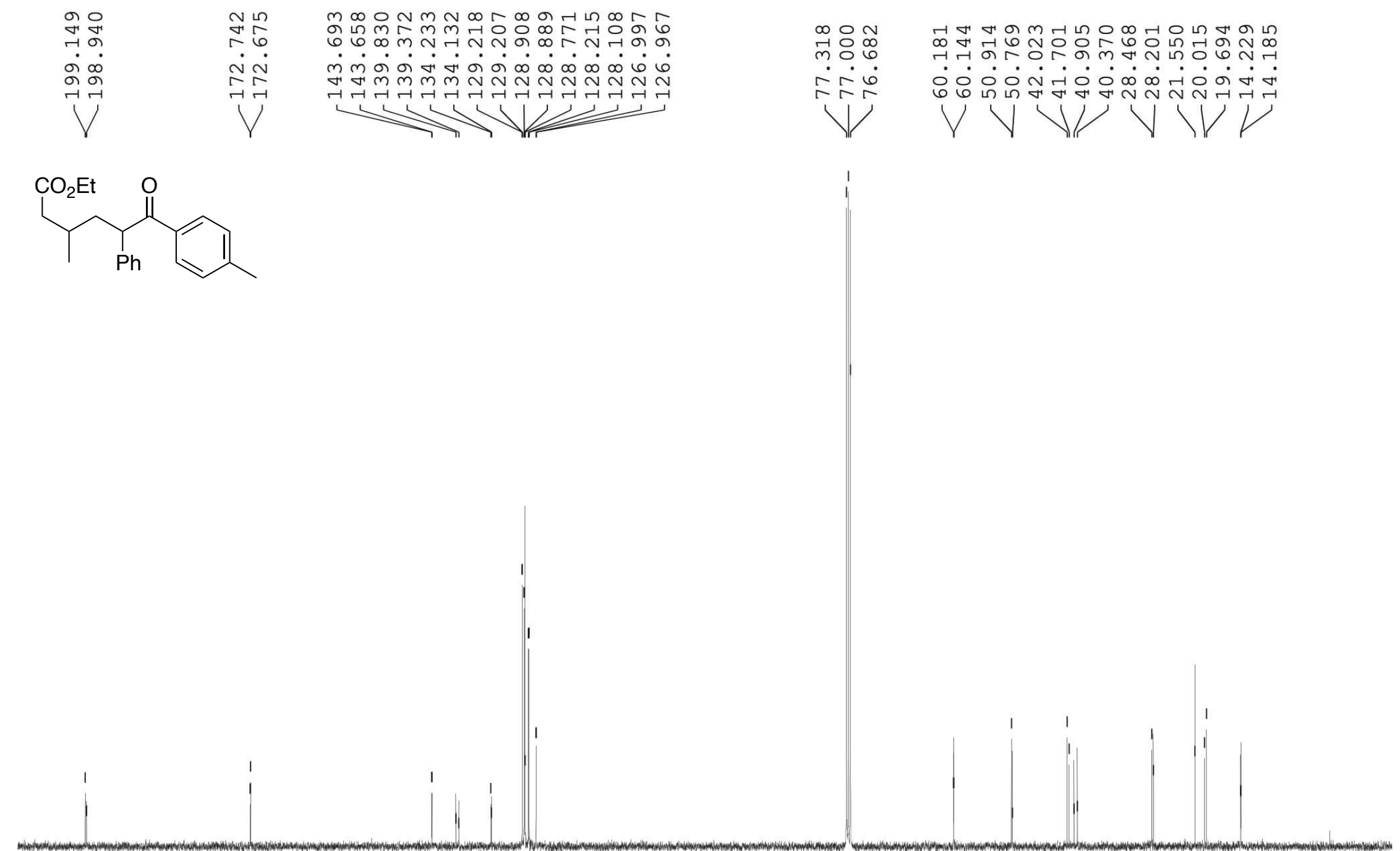

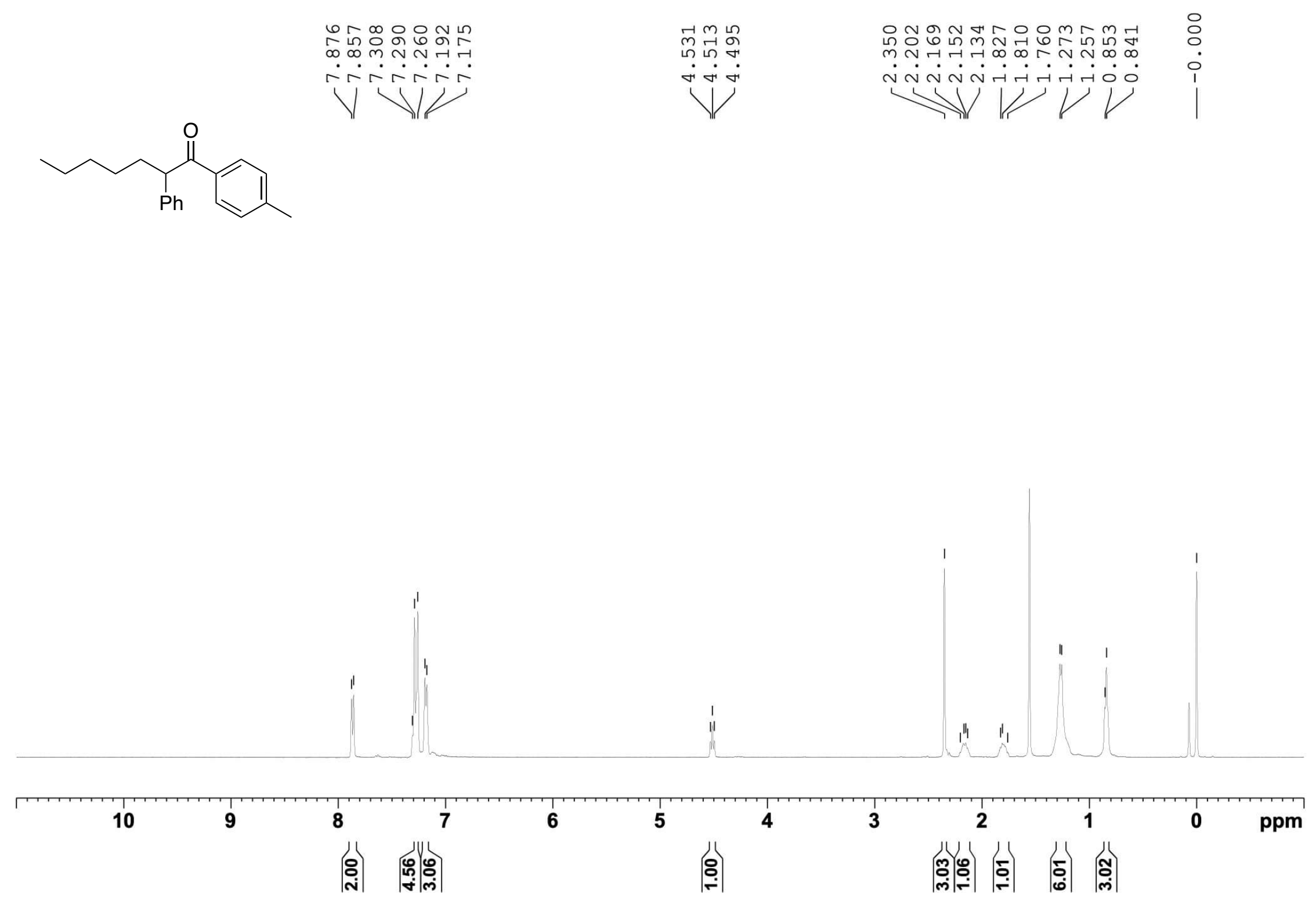

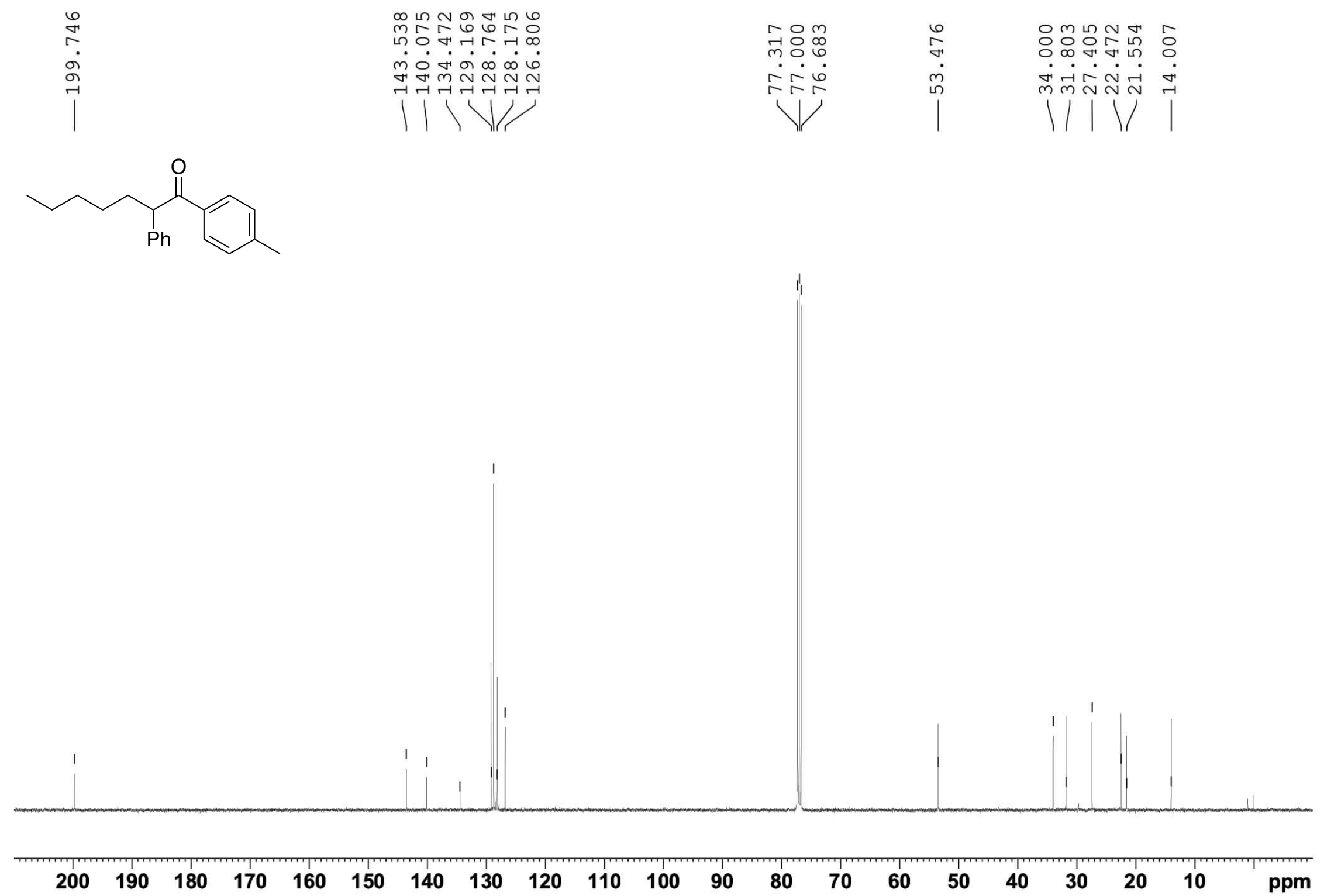


\section{${ }^{1} \mathrm{H}$ NMR of $\mathbf{6 f a a}$}

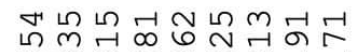
$\infty \infty m \sim N N$ ririsisitr

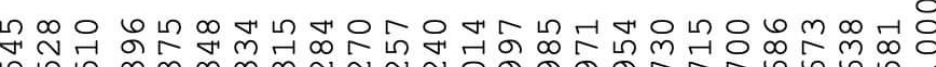
Vi
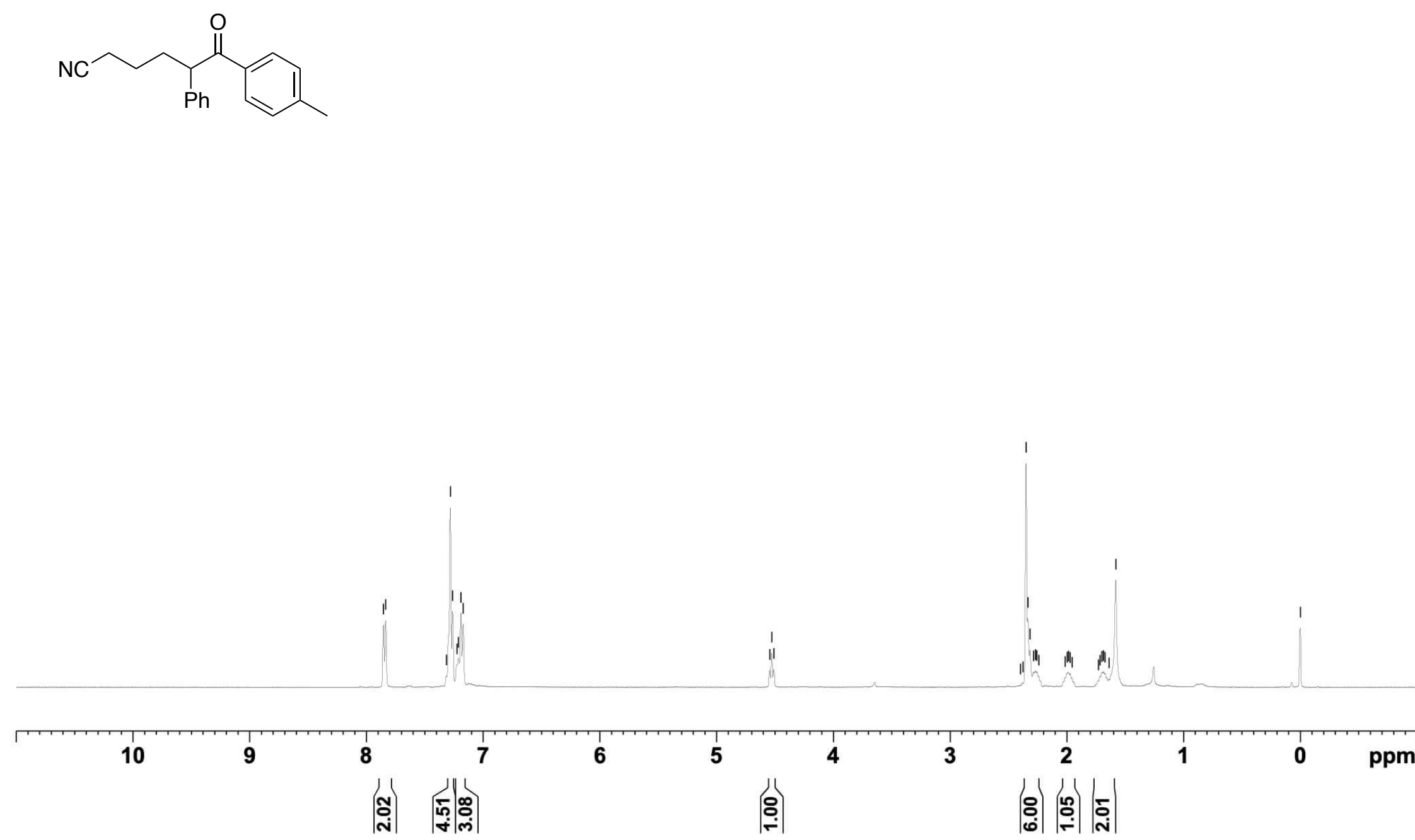

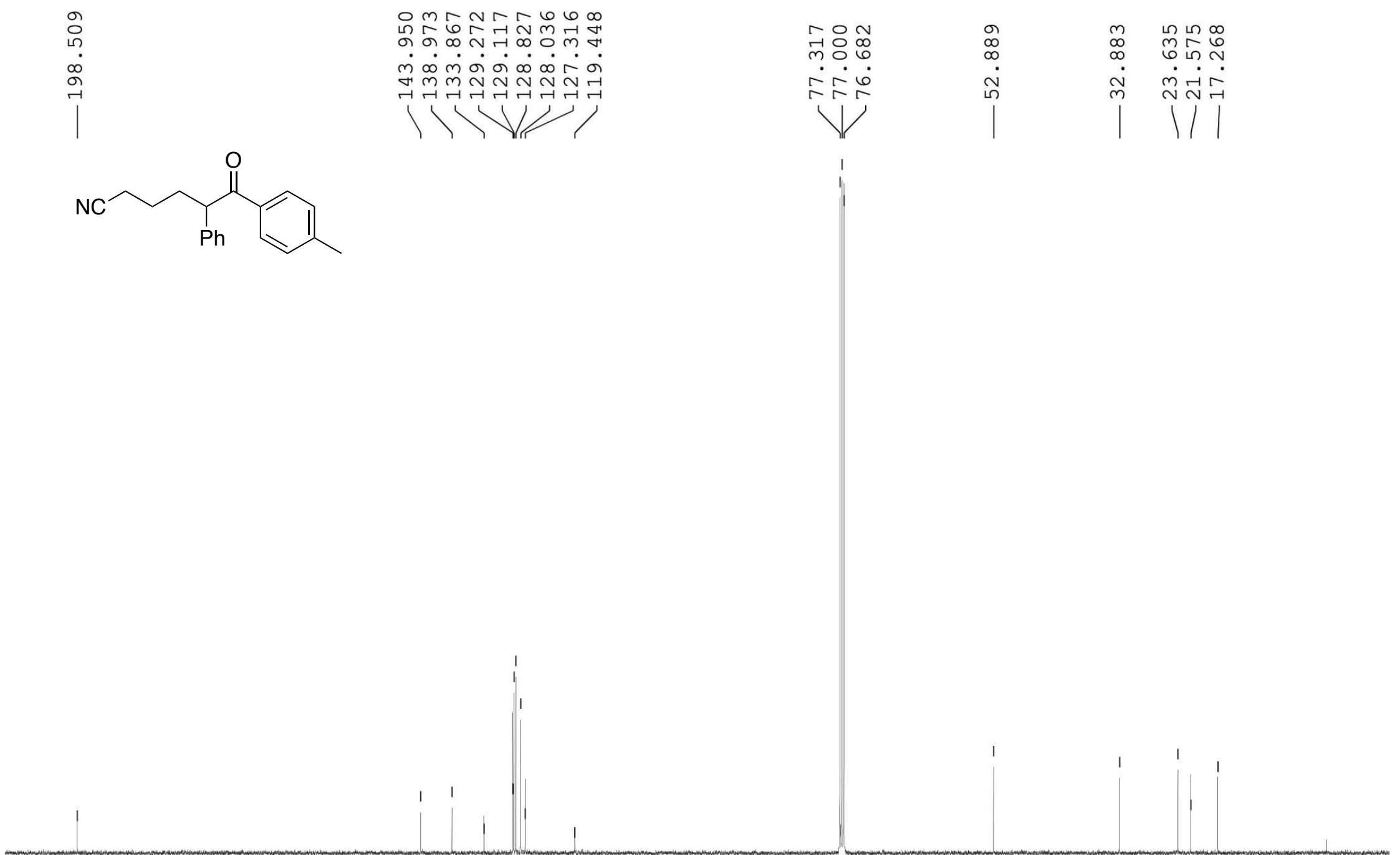

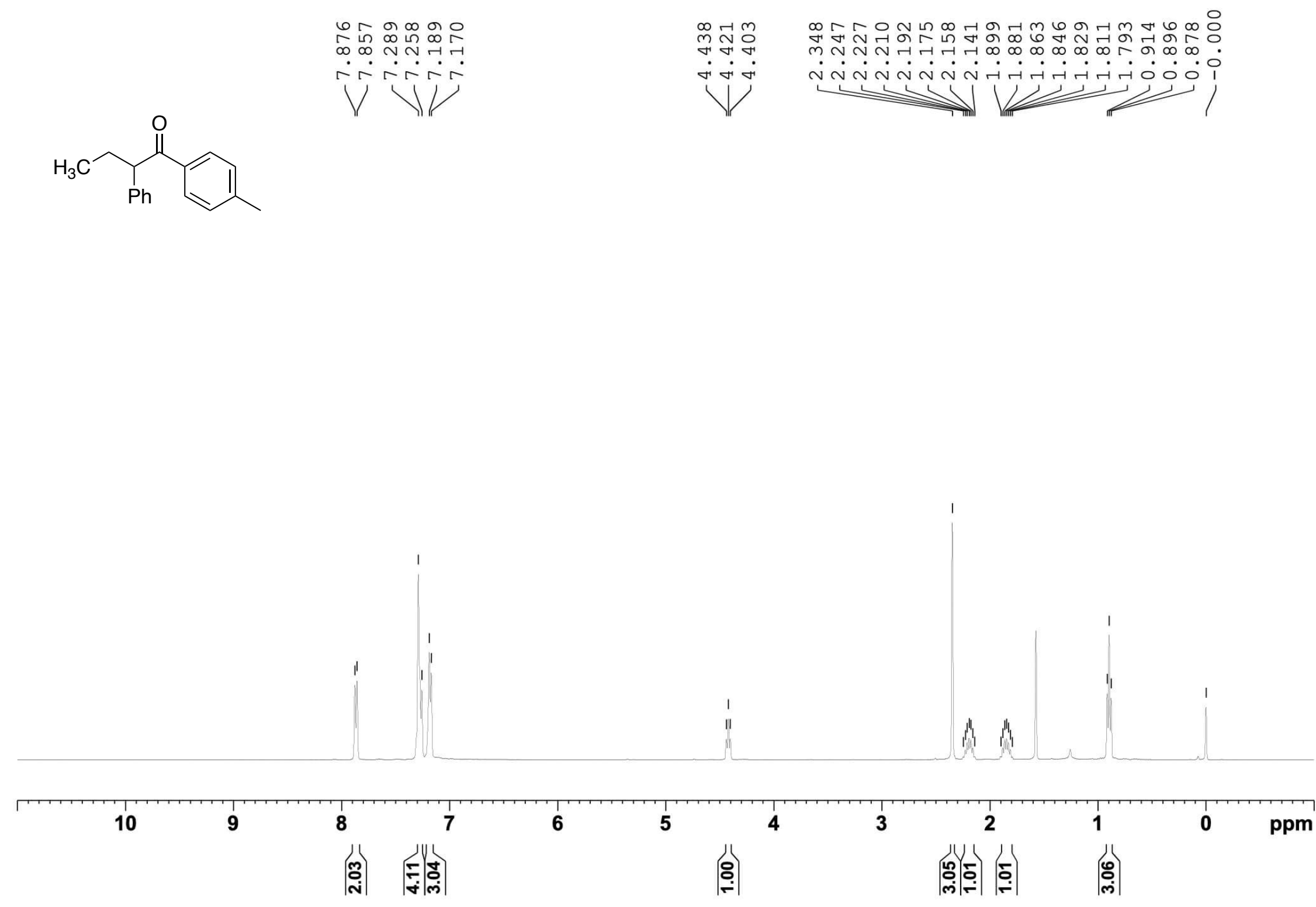

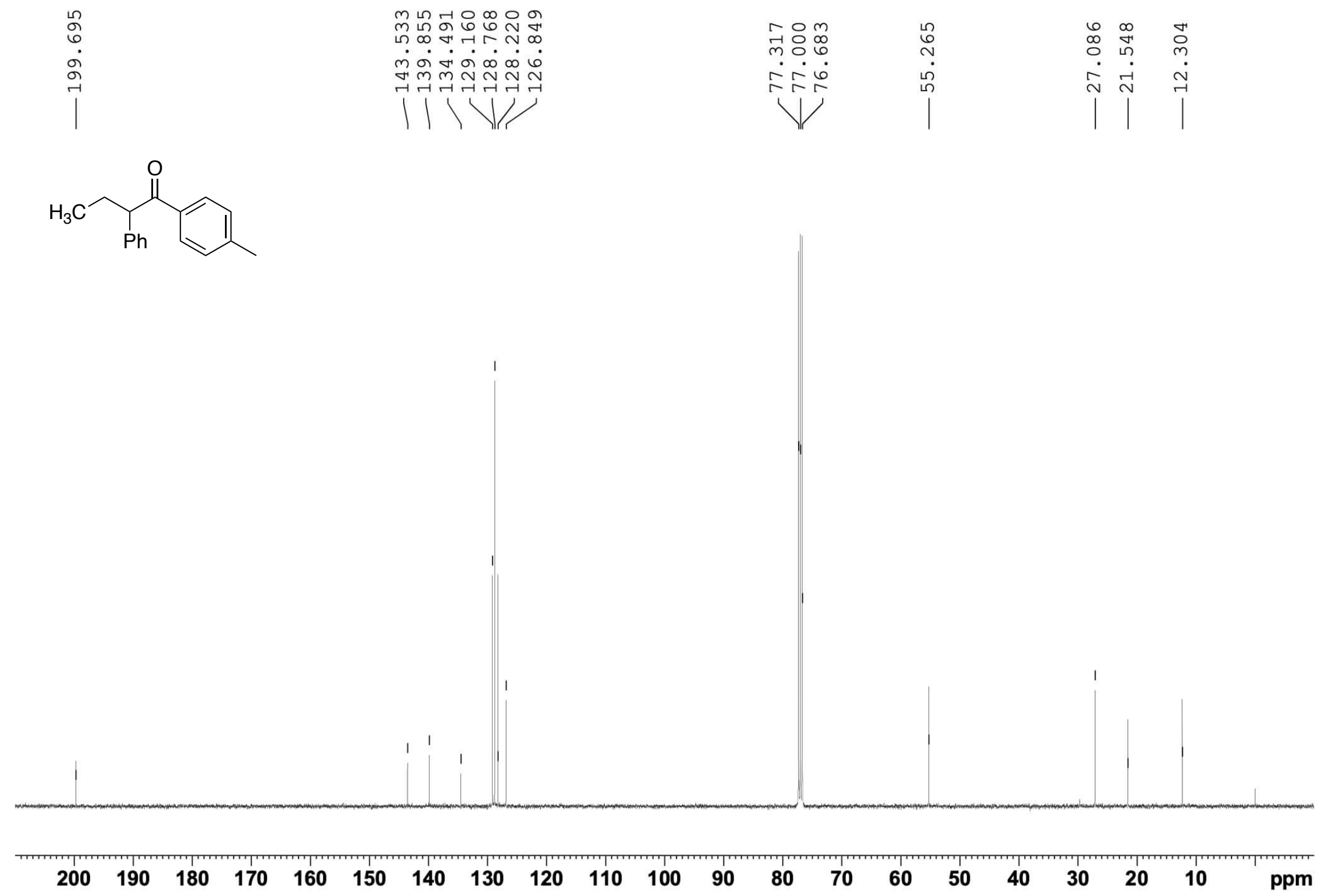

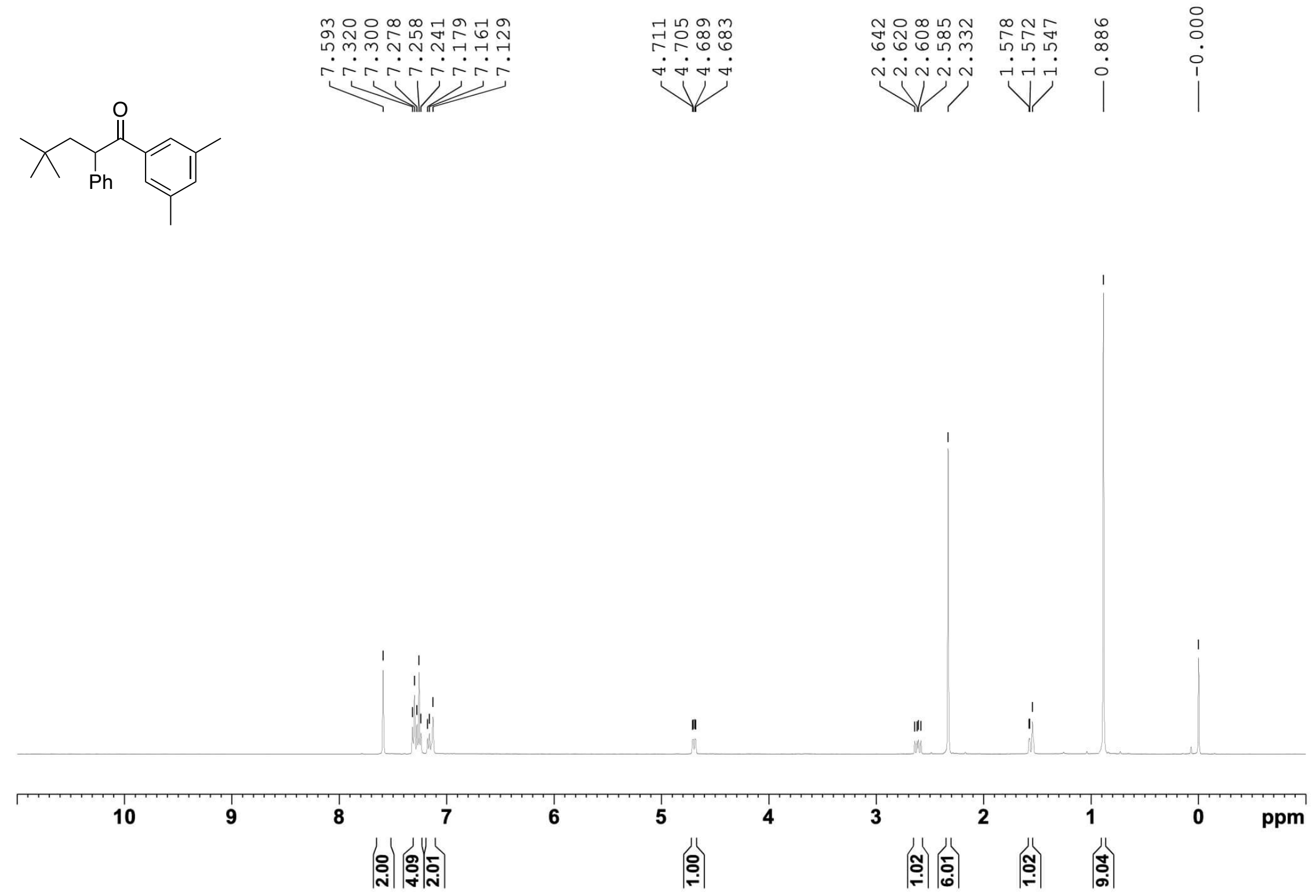

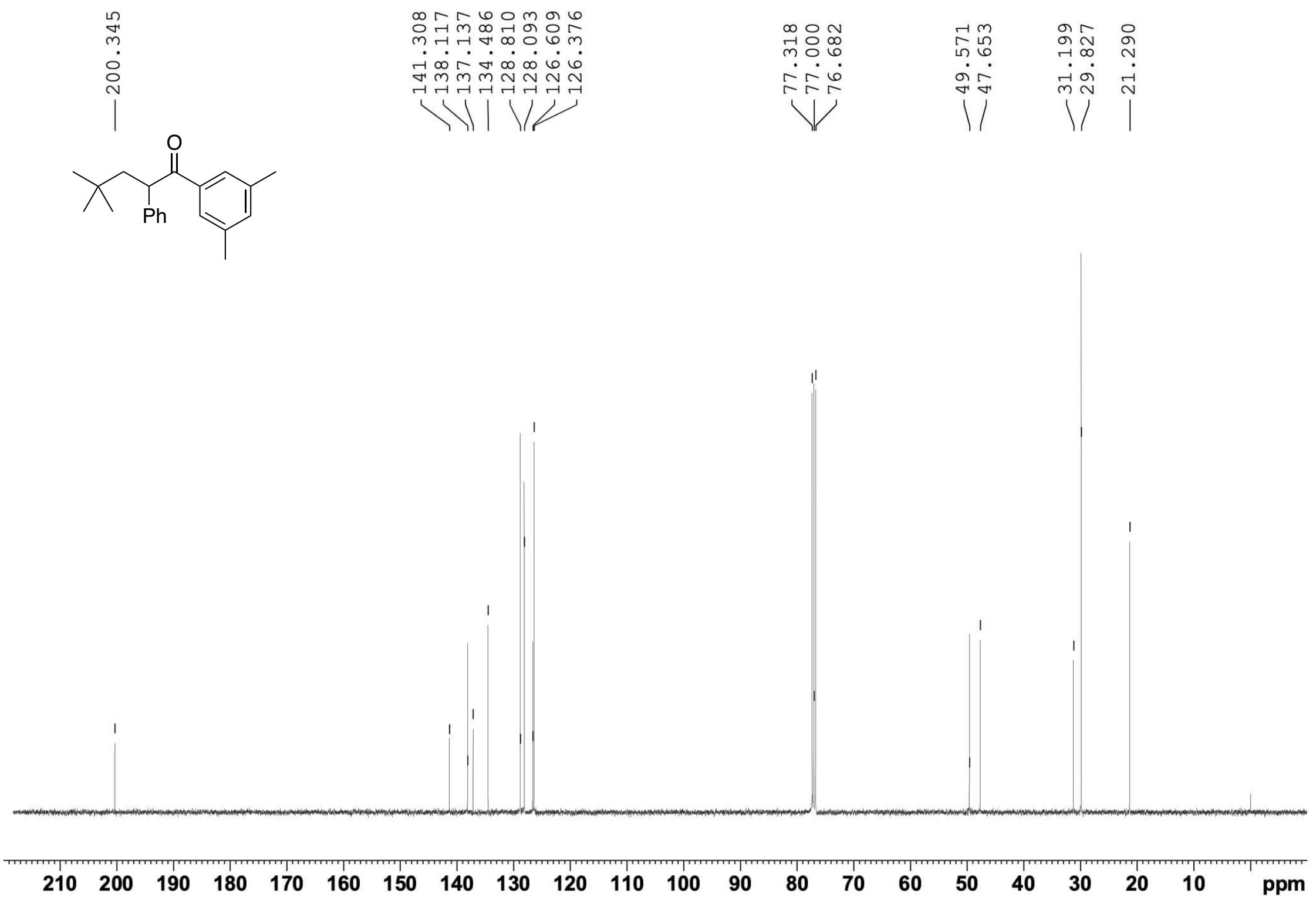
돈

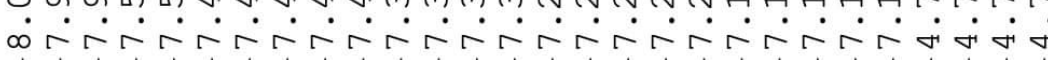

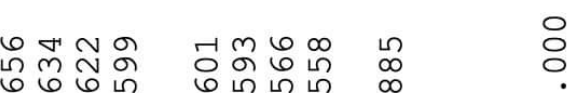

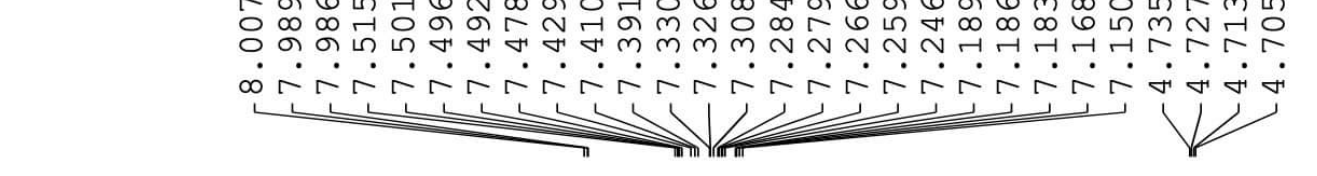
$\overbrace{P h}^{O}$ $\dot{\mathrm{i}} \dot{\mathrm{i}}$ vi
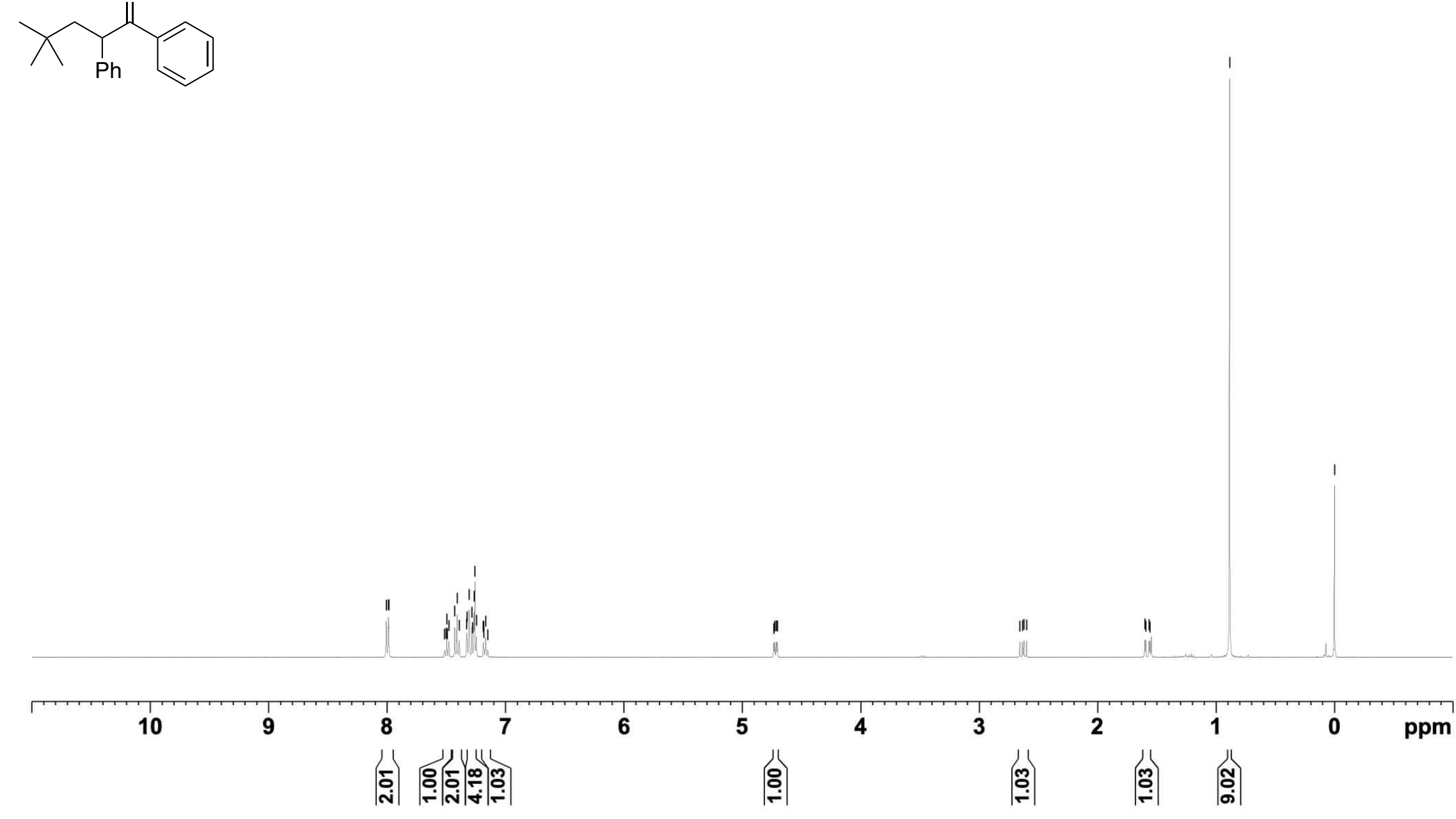
${ }^{13} \mathrm{C}$ NMR of $6 \mathbf{a a j}$

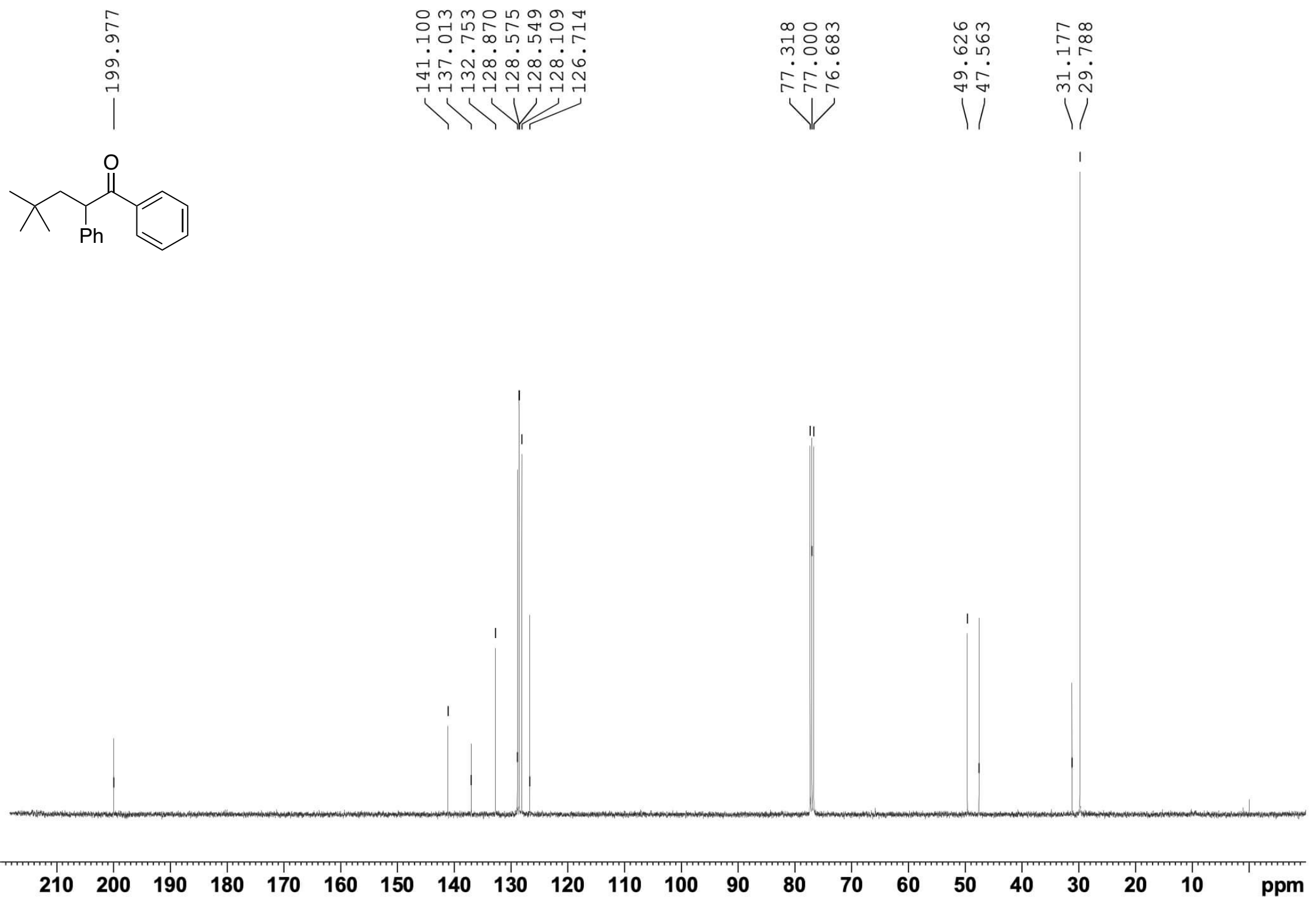




\section{${ }^{1} \mathrm{H}$ NMR of $\mathbf{6 a a g}$}
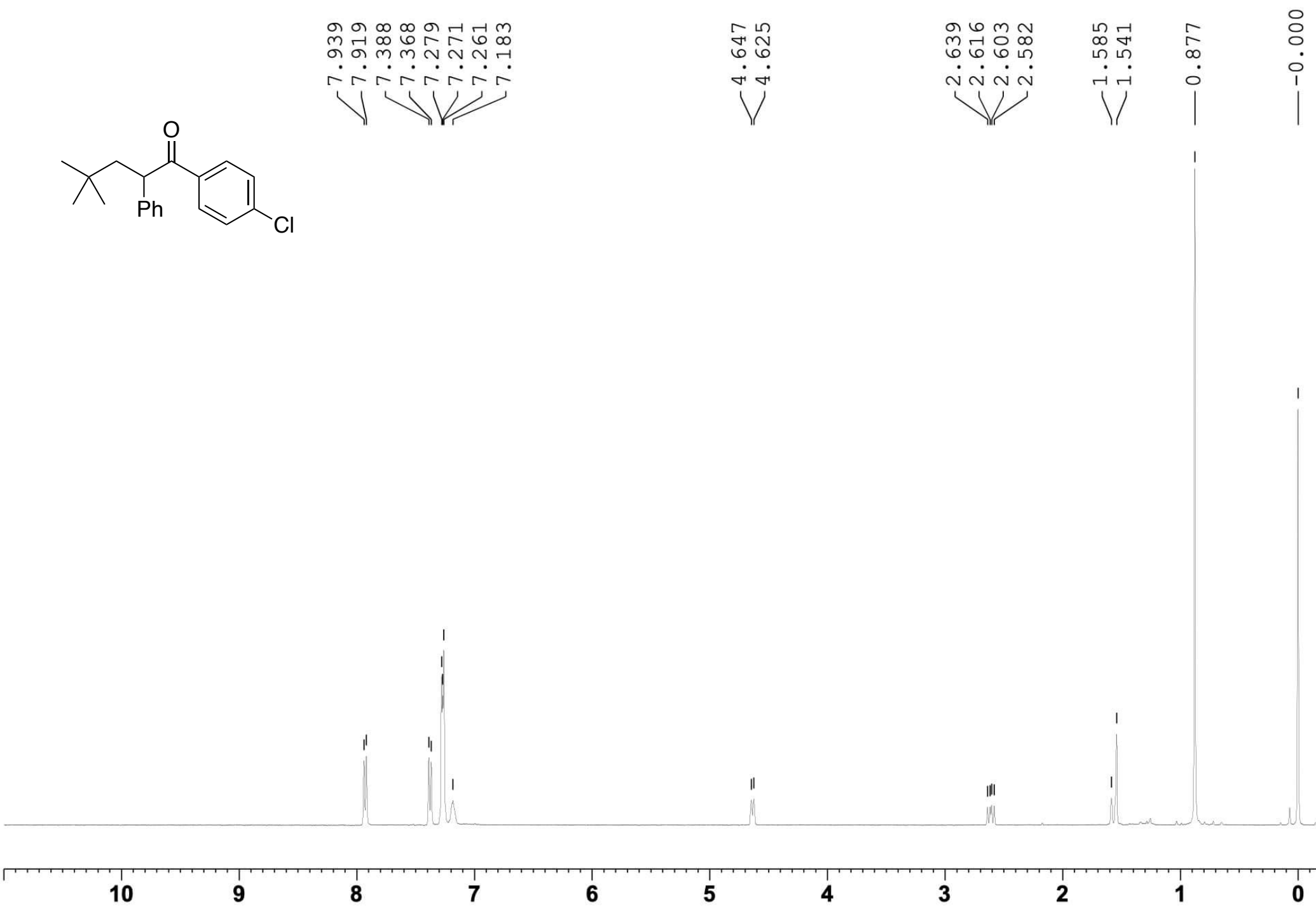

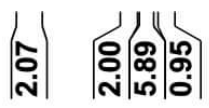

7

6

5

$\mid$

3

$\left|\begin{array}{l}\mid \\ \vdots \\ \hdashline\end{array}\right|$

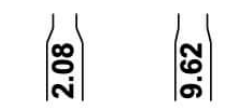

ppm 

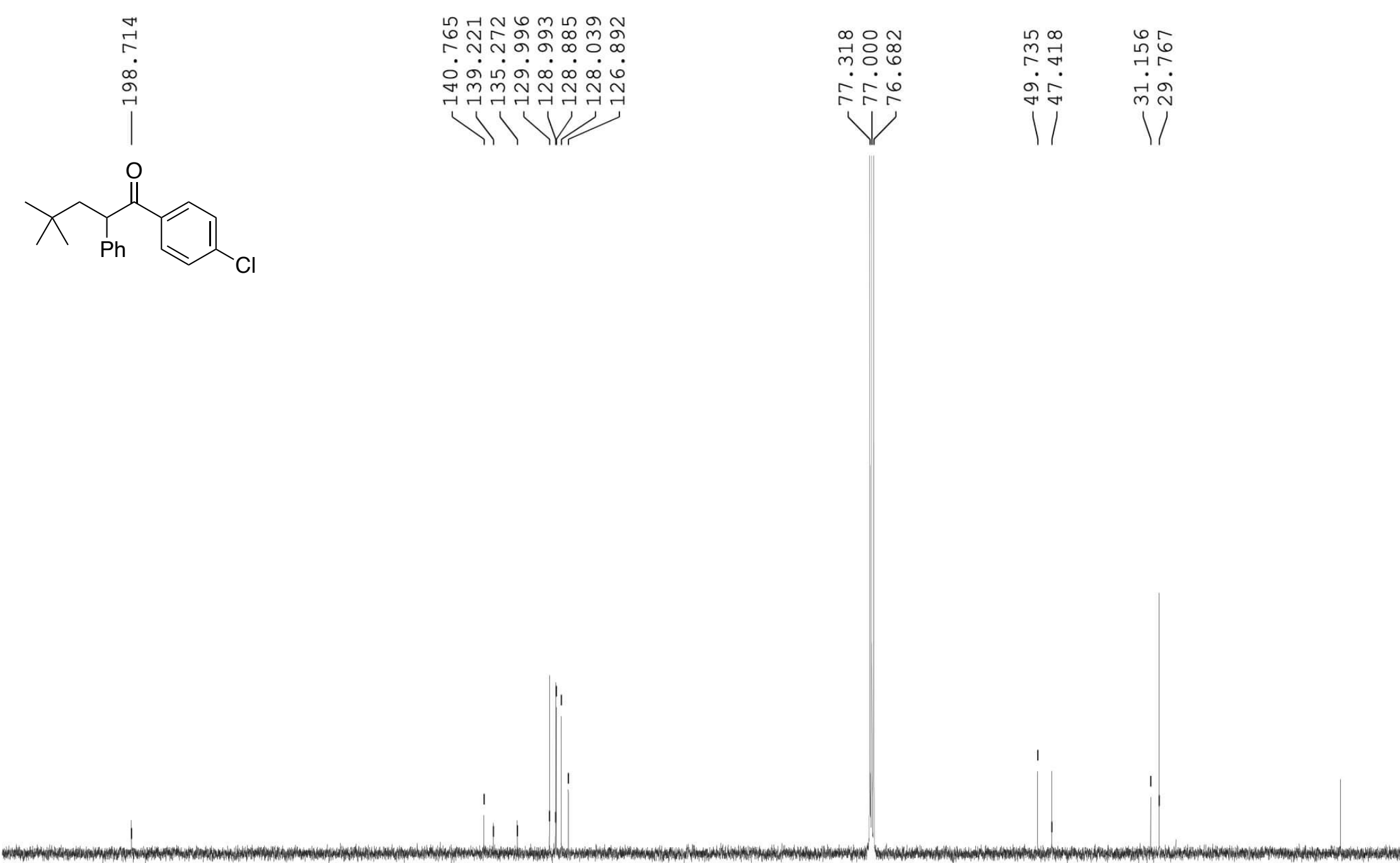

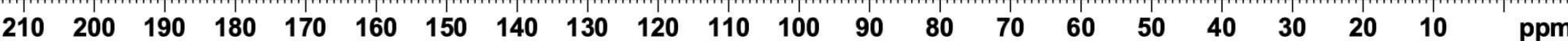


${ }^{1} \mathrm{H}$ NMR of $\mathbf{6 a a k}$
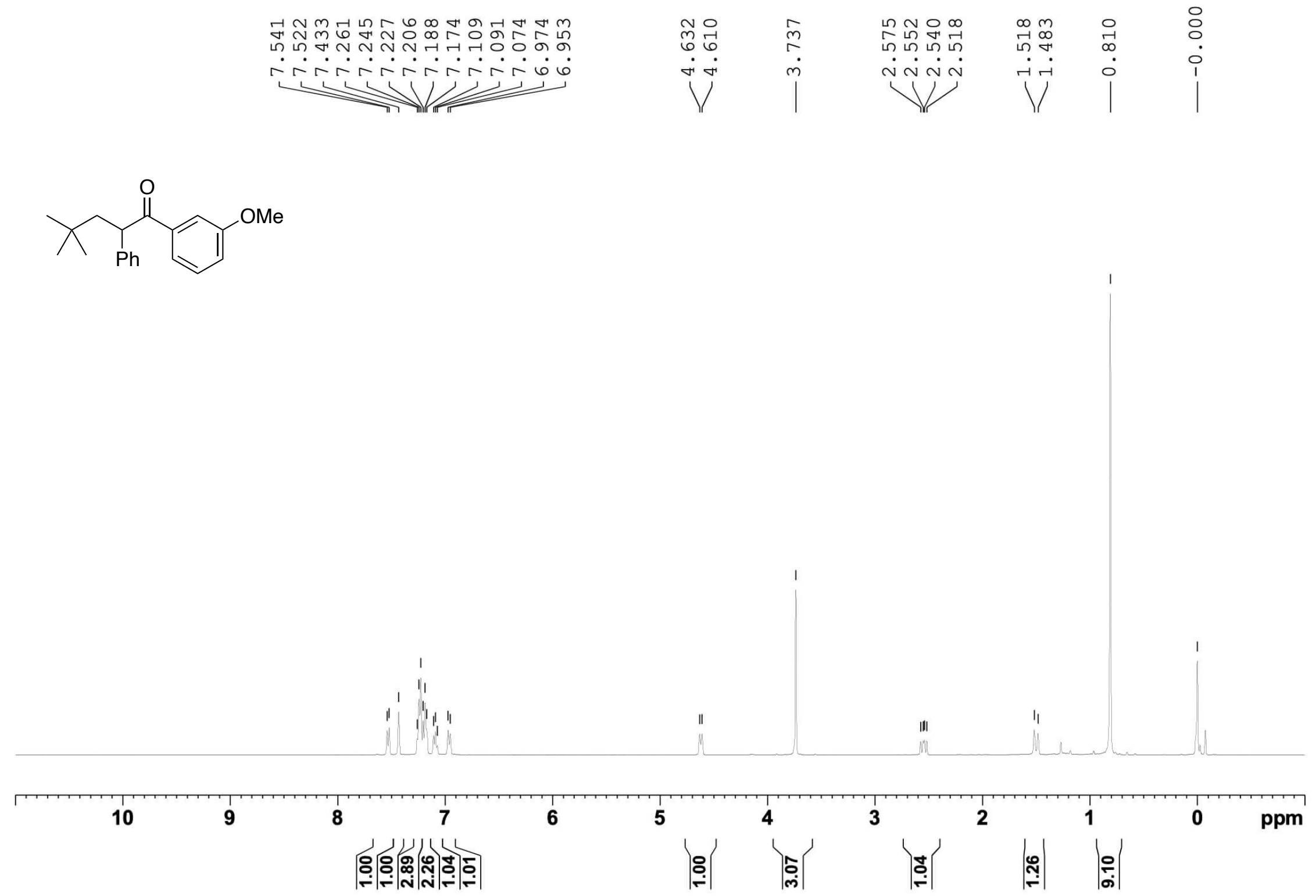


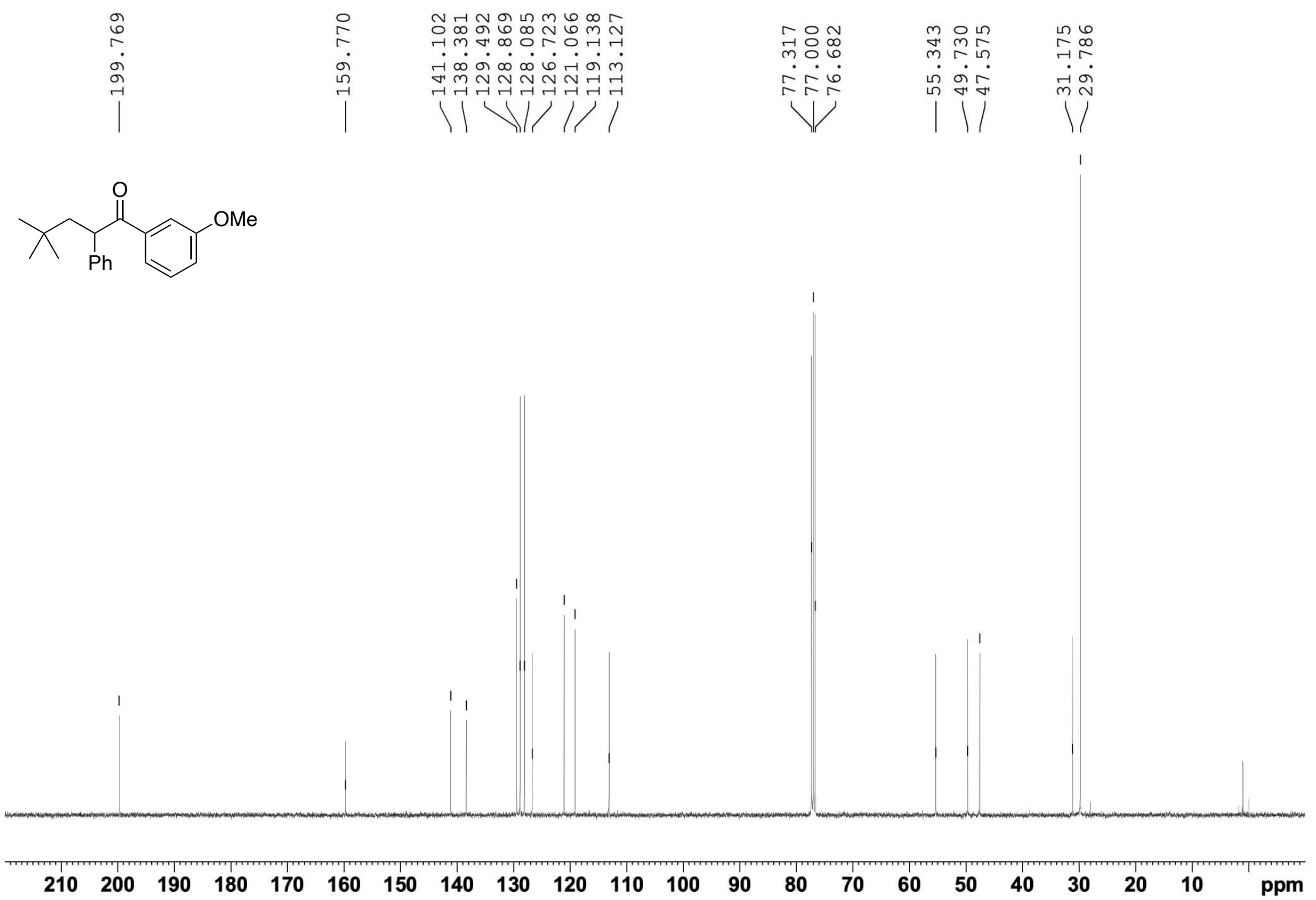




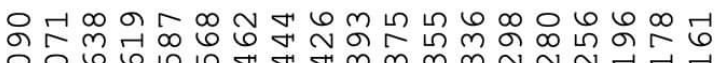

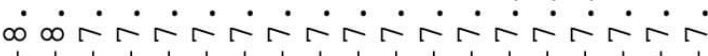

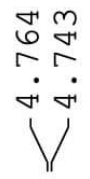

అ

$\dot{\sim} \dot{\sim} \dot{\sim} \dot{N}$

W
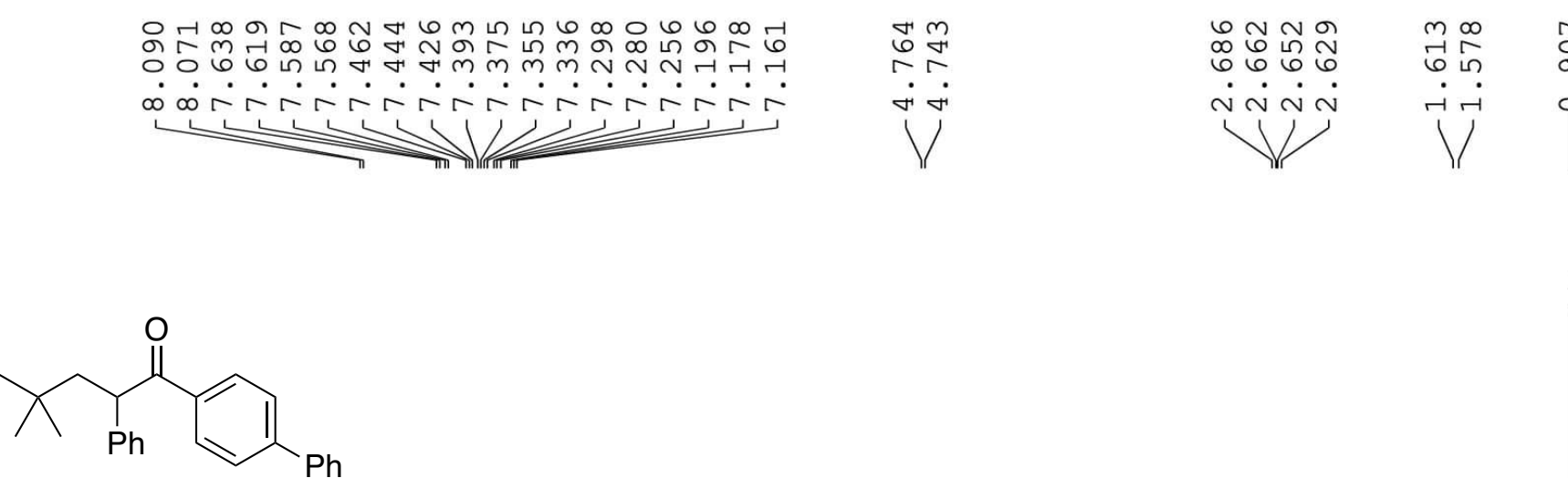

$\mathrm{Ph}$

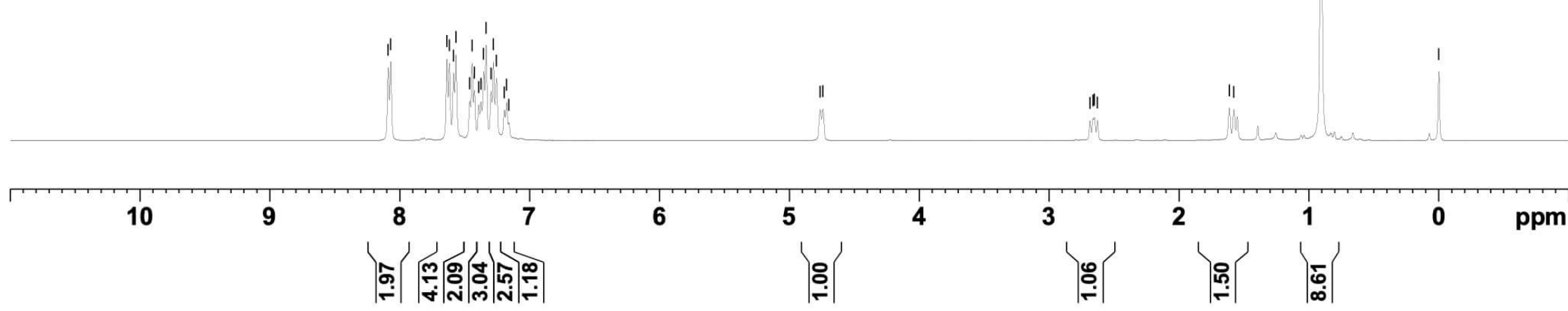




\section{\begin{tabular}{l}
$\infty$ \\
$\stackrel{+}{+}$ \\
$\circ$ \\
$\stackrel{2}{7}$ \\
\multirow{1}{1}{}
\end{tabular}}

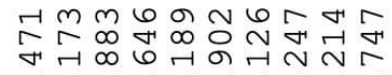

नं

HMm N N N N

1)

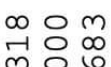

둥.

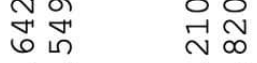

नुं मंब

Vij

$1 / 1$
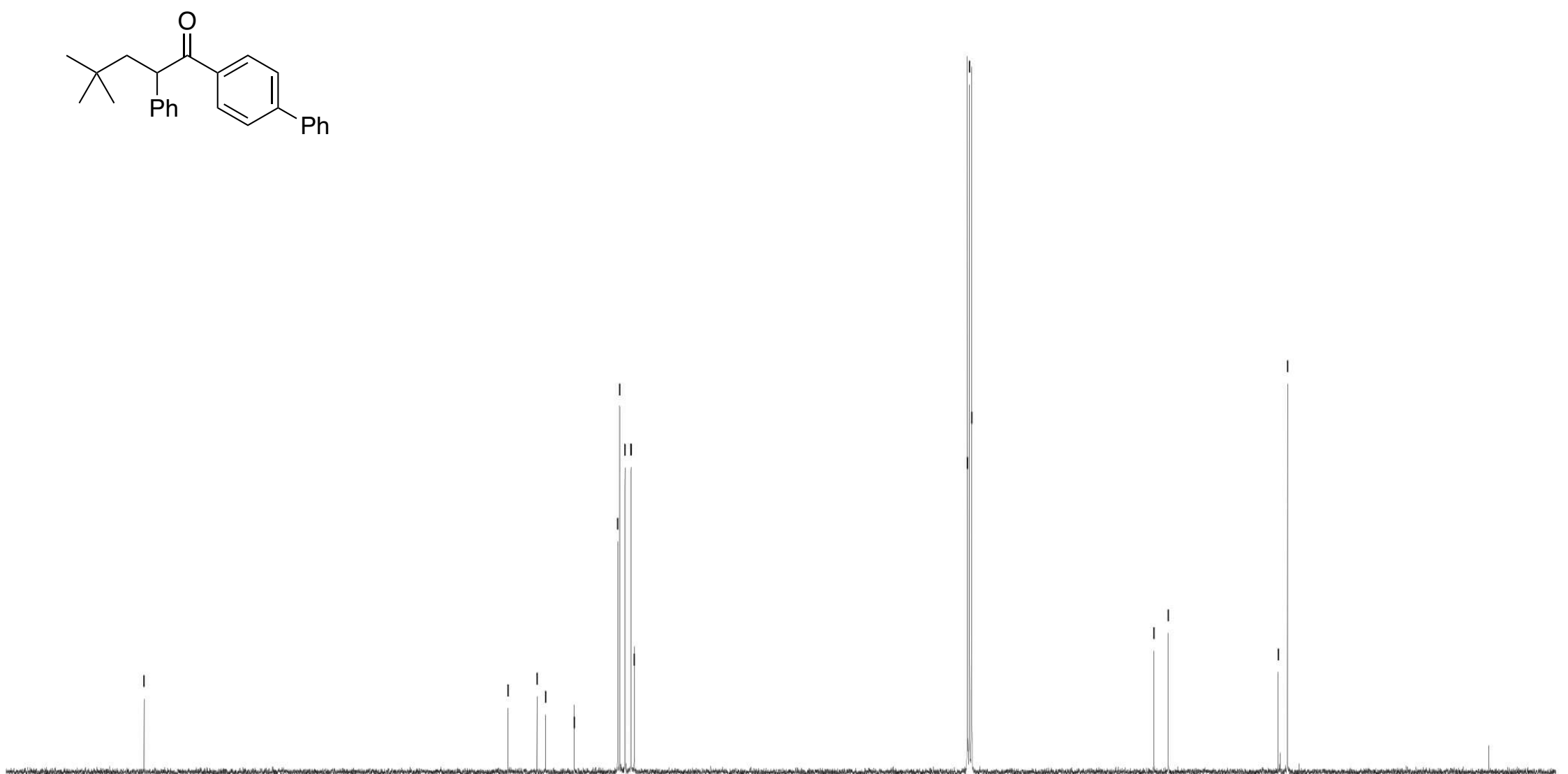

210200190 


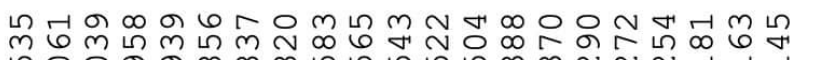

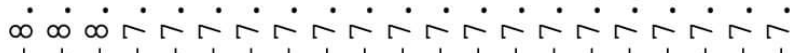

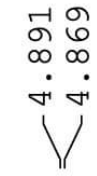

인

$\dot{\sim} \dot{\sim} \dot{j}$

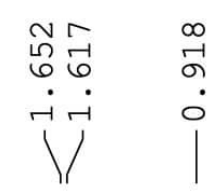

$\circ$
0
0
1
1
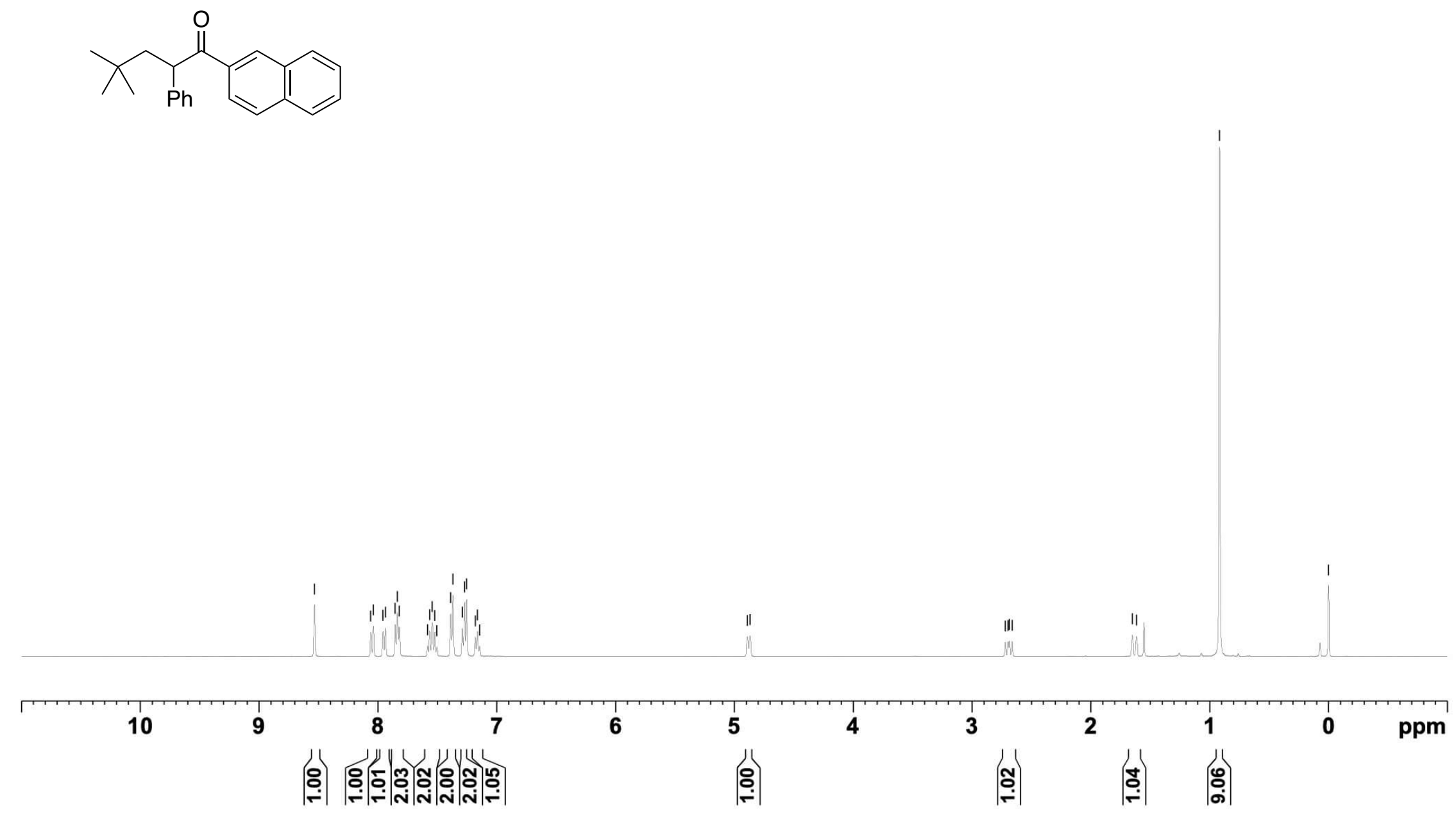

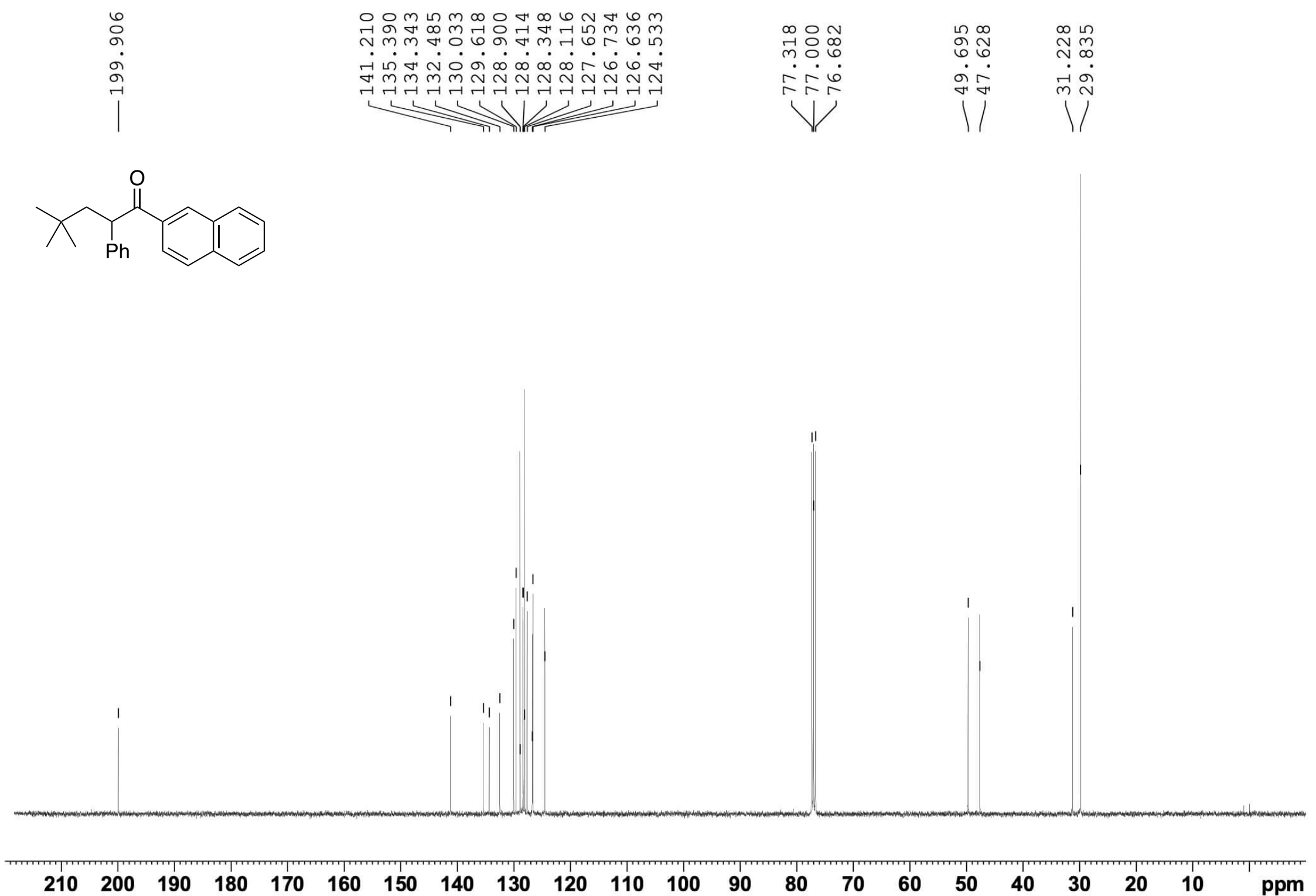

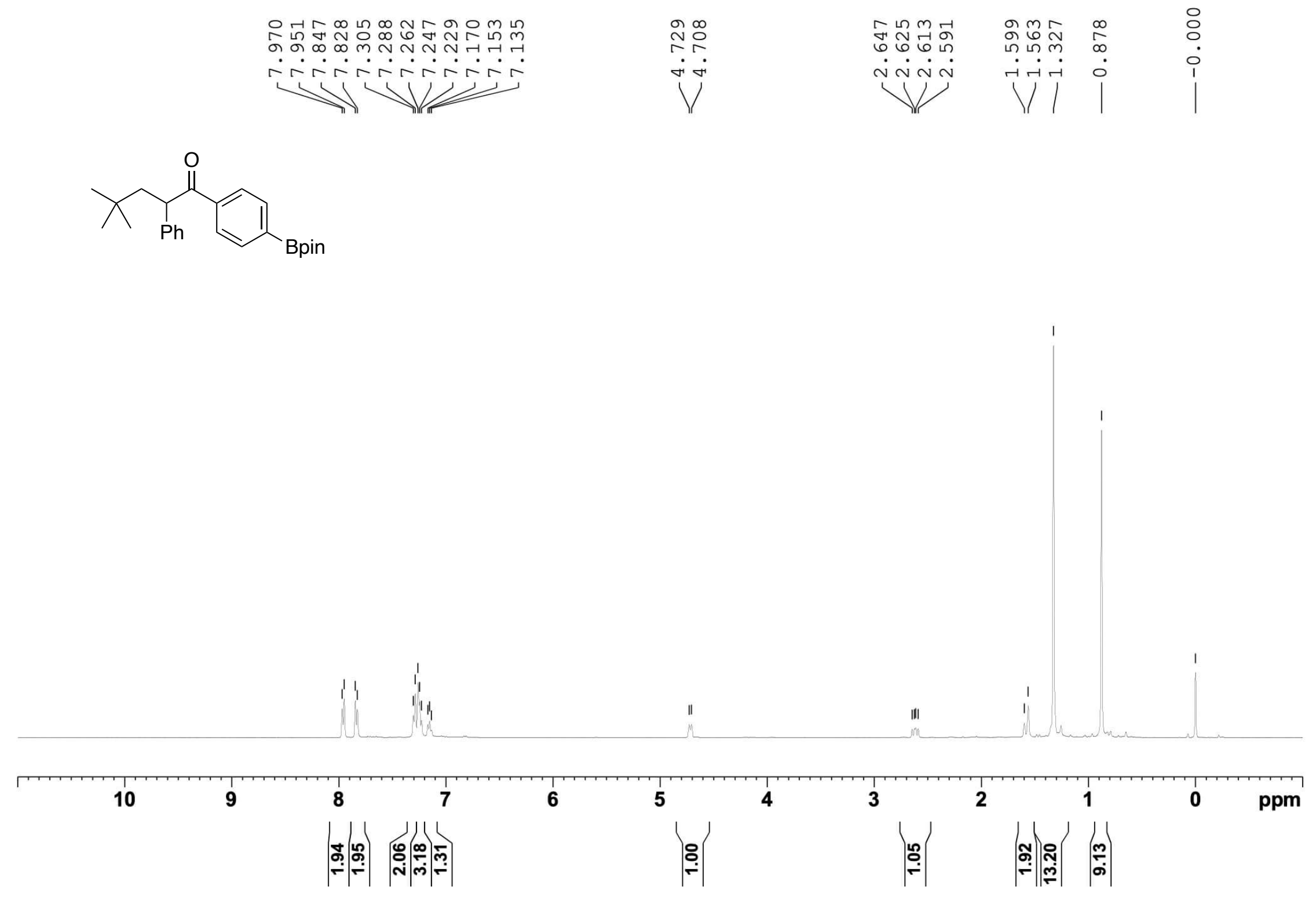

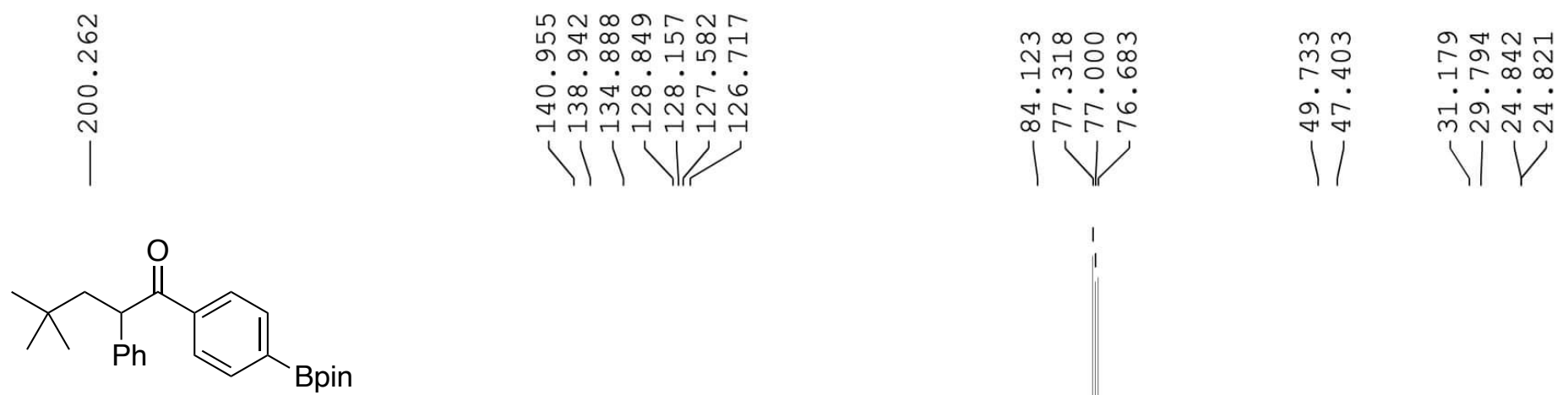

Bpin

$\begin{array}{llllllllllll}210 & 200 & 190 & 180 & 170 & 160 & 150 & 140 & 130 & 120 & 110 & 100\end{array}$ 

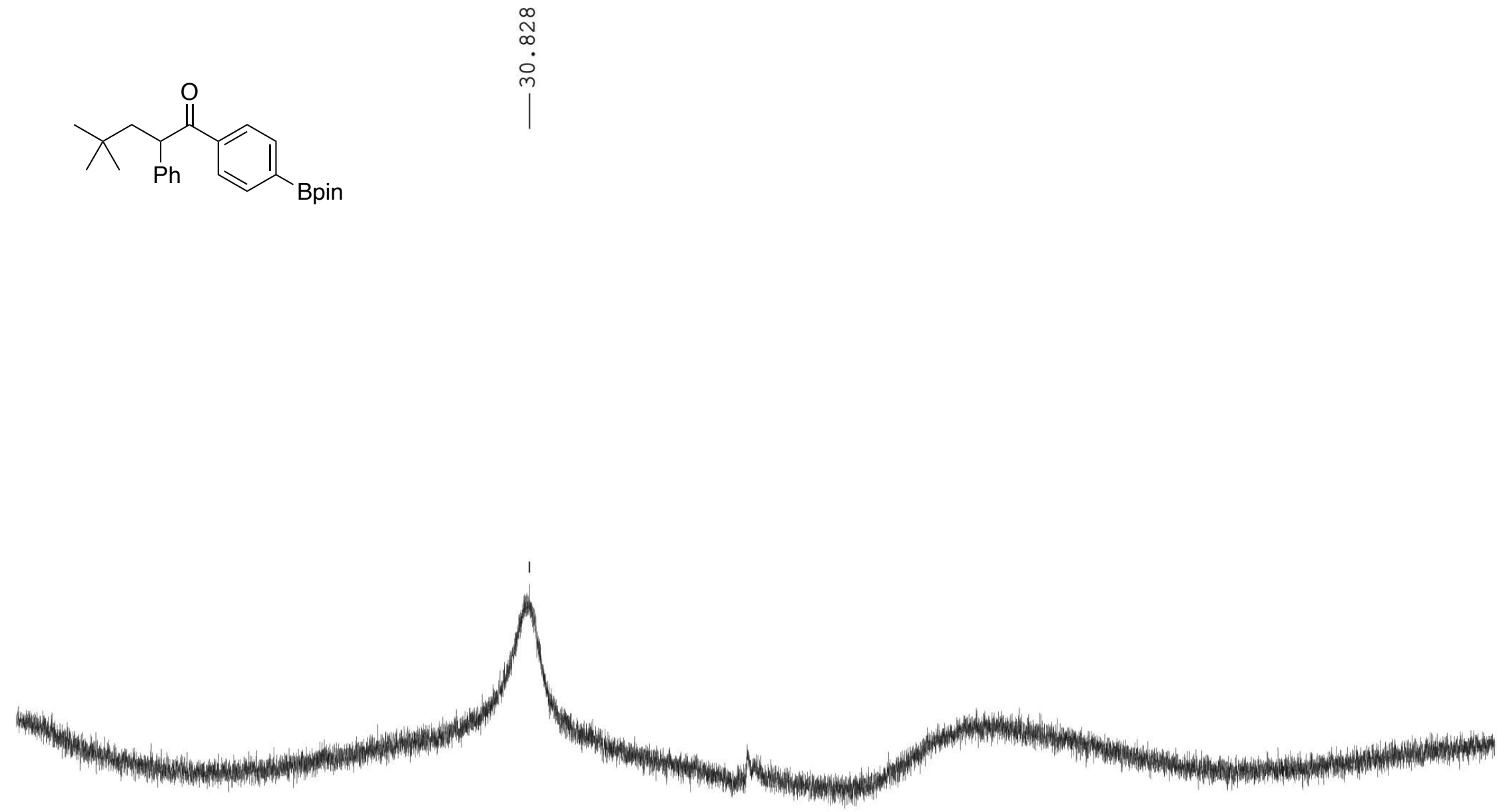


\section{${ }^{1} \mathrm{H}$ NMR of 6 aam}

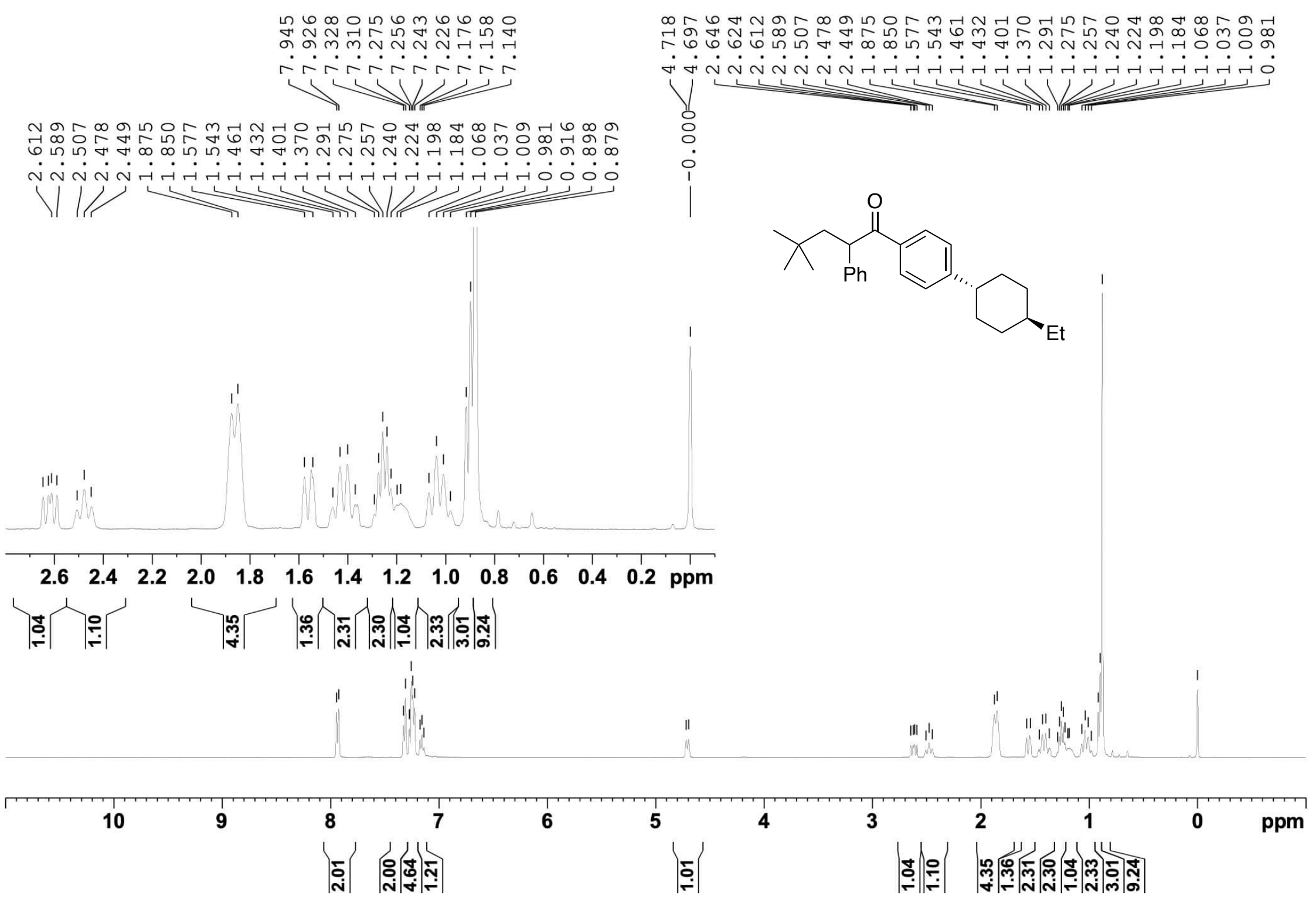



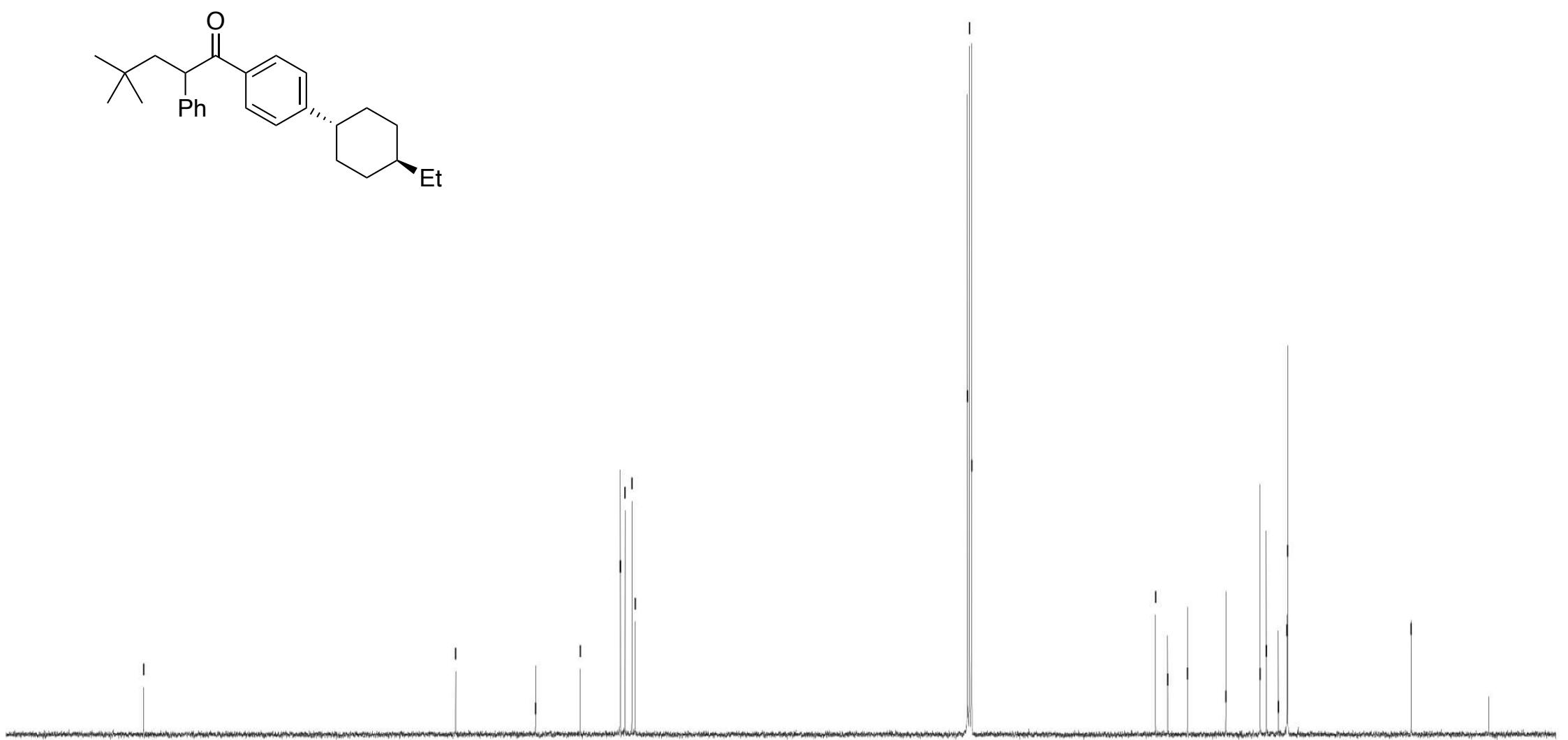


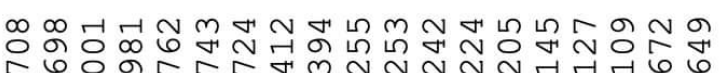

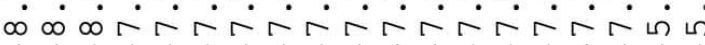

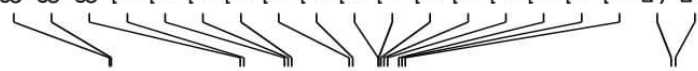

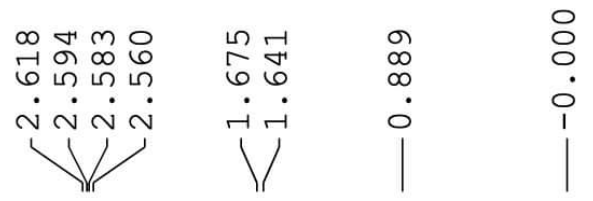
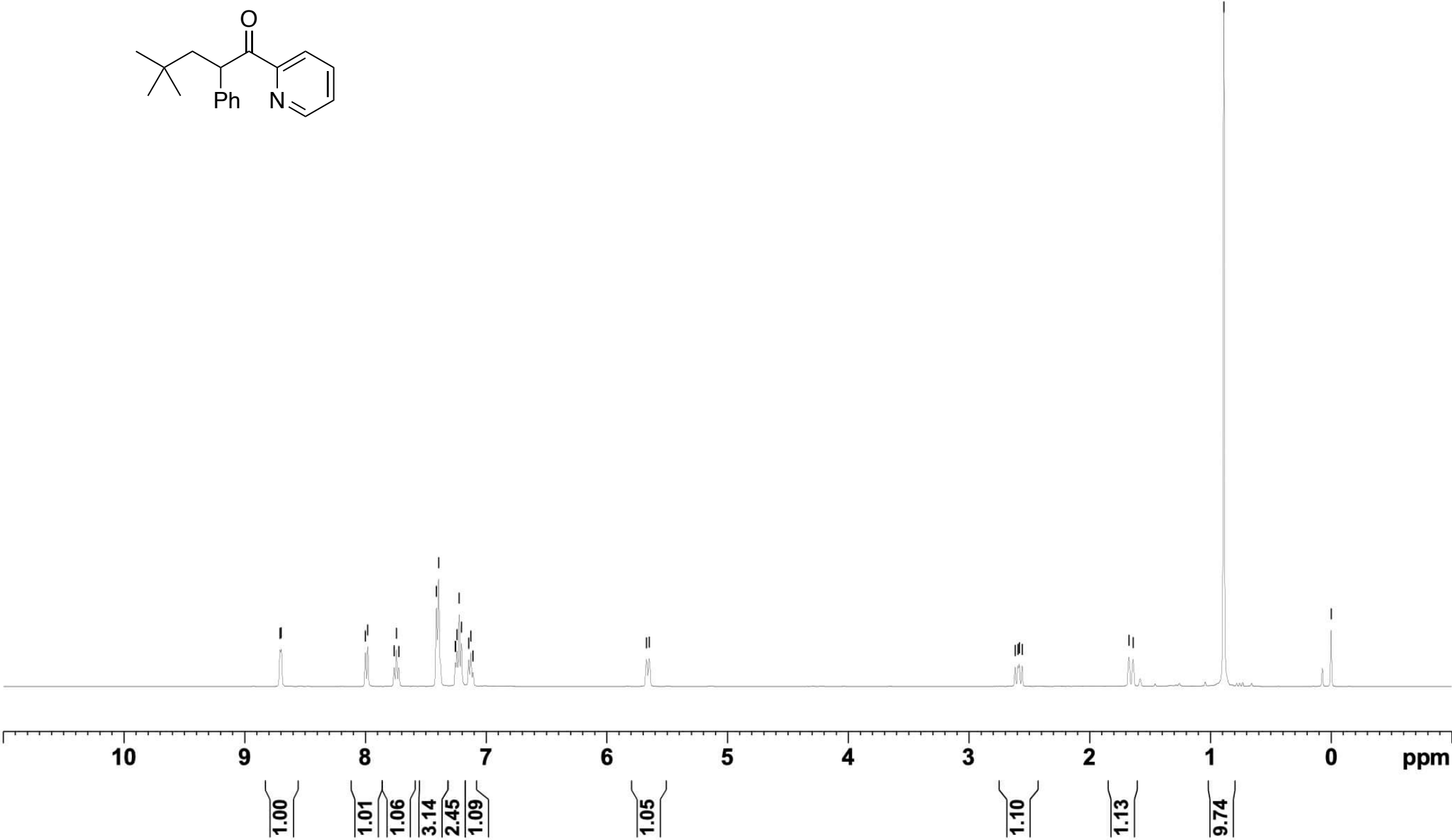

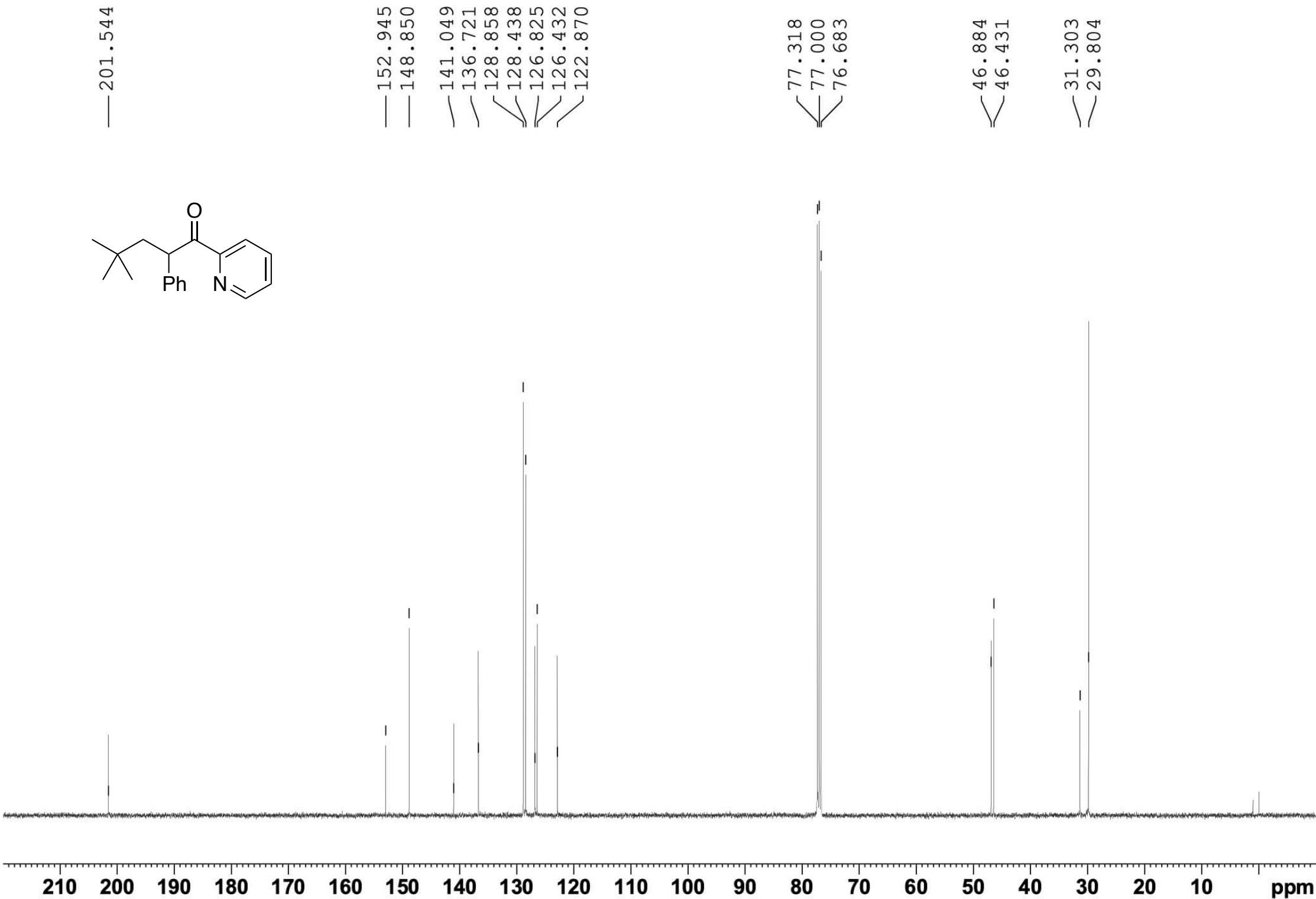


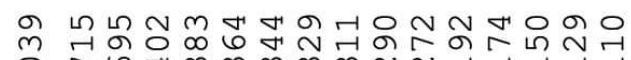

arririrarinariar

$\longrightarrow$

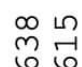

i

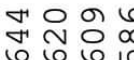

نั่งن

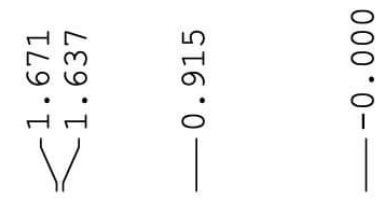
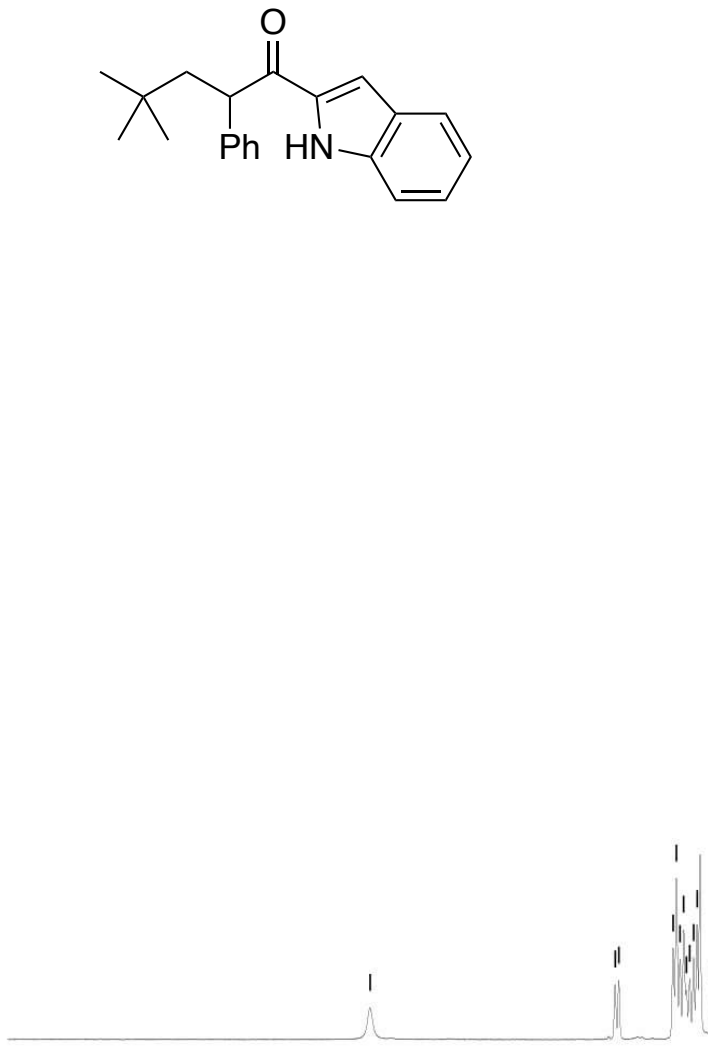

$\left|\begin{array}{c}9 \\ \hdashline \\ 0 \\ 0\end{array}\right|$

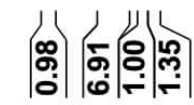

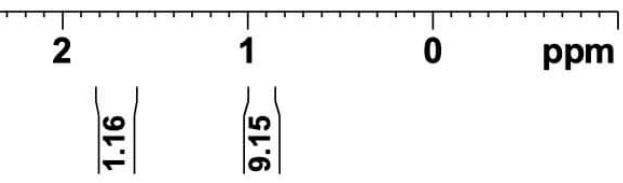




\section{मे
ने
ने
|}

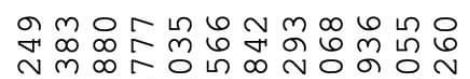

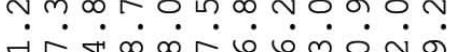

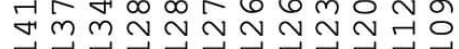

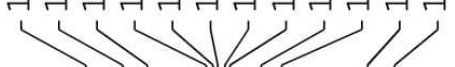
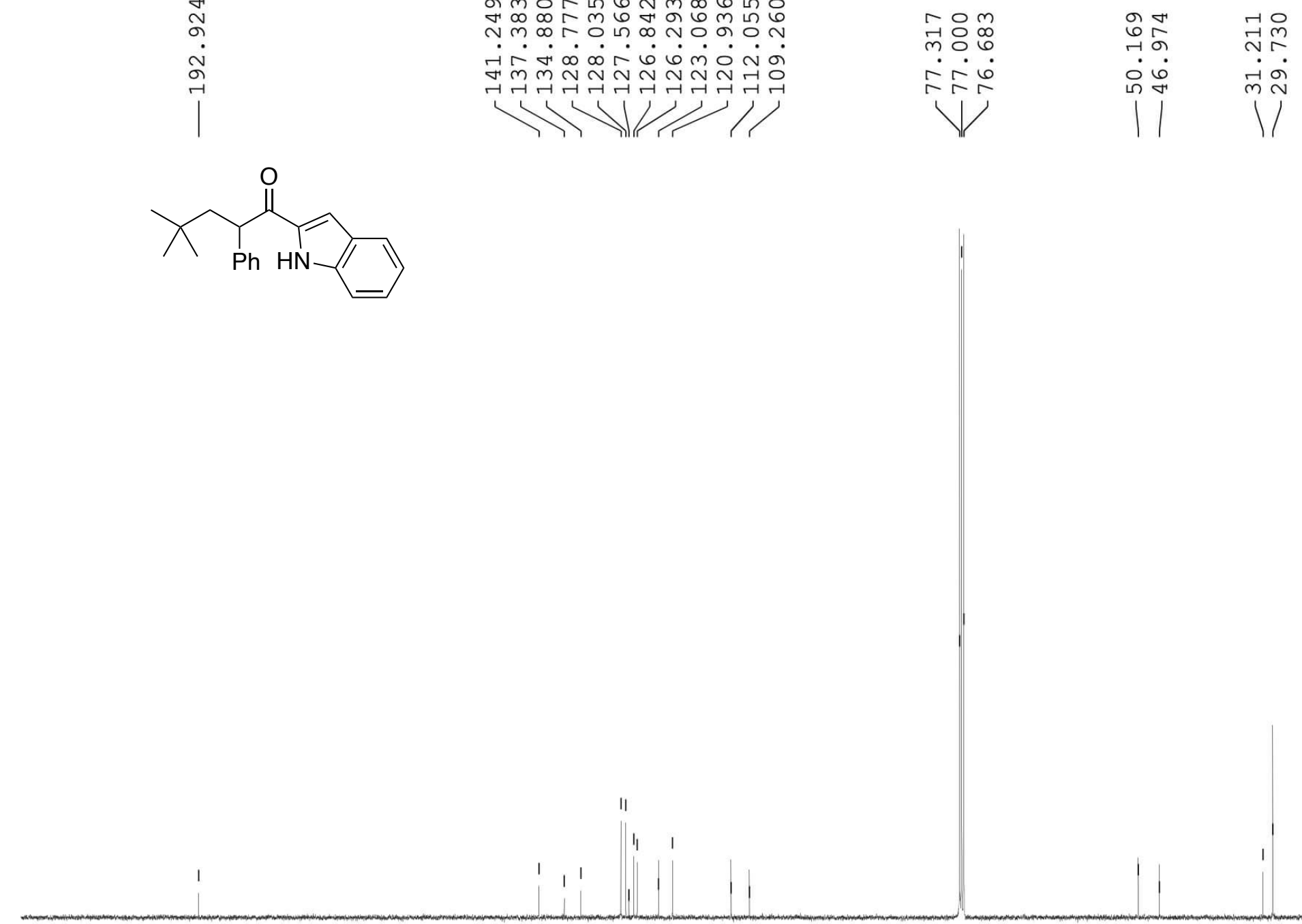

210200190

$\begin{array}{llll}180 & 170 \quad 160 \quad 1\end{array}$

140

$\begin{array}{llll}130 & 120 & 110 & 100\end{array}$

90

80

70

60

40

30 

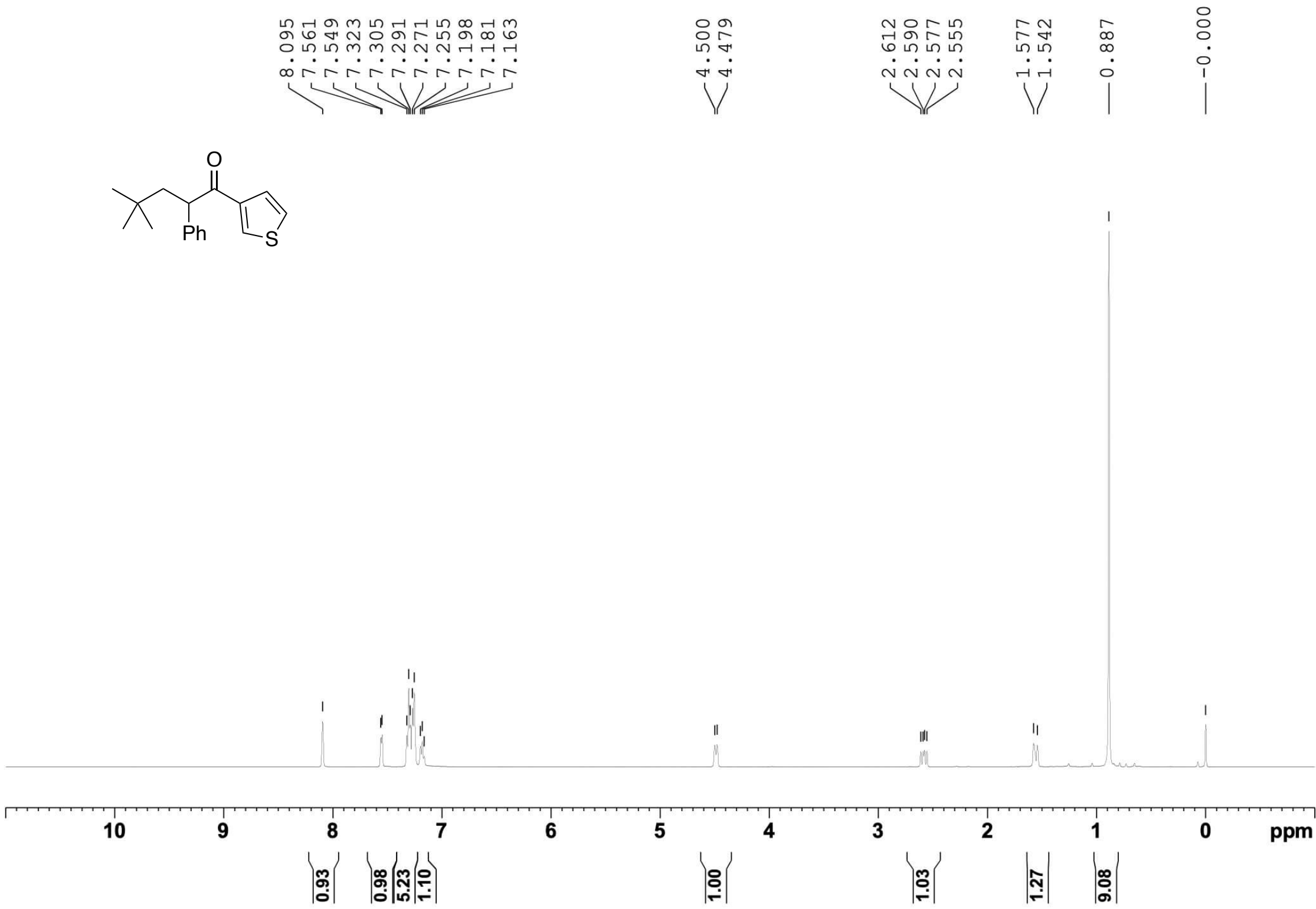

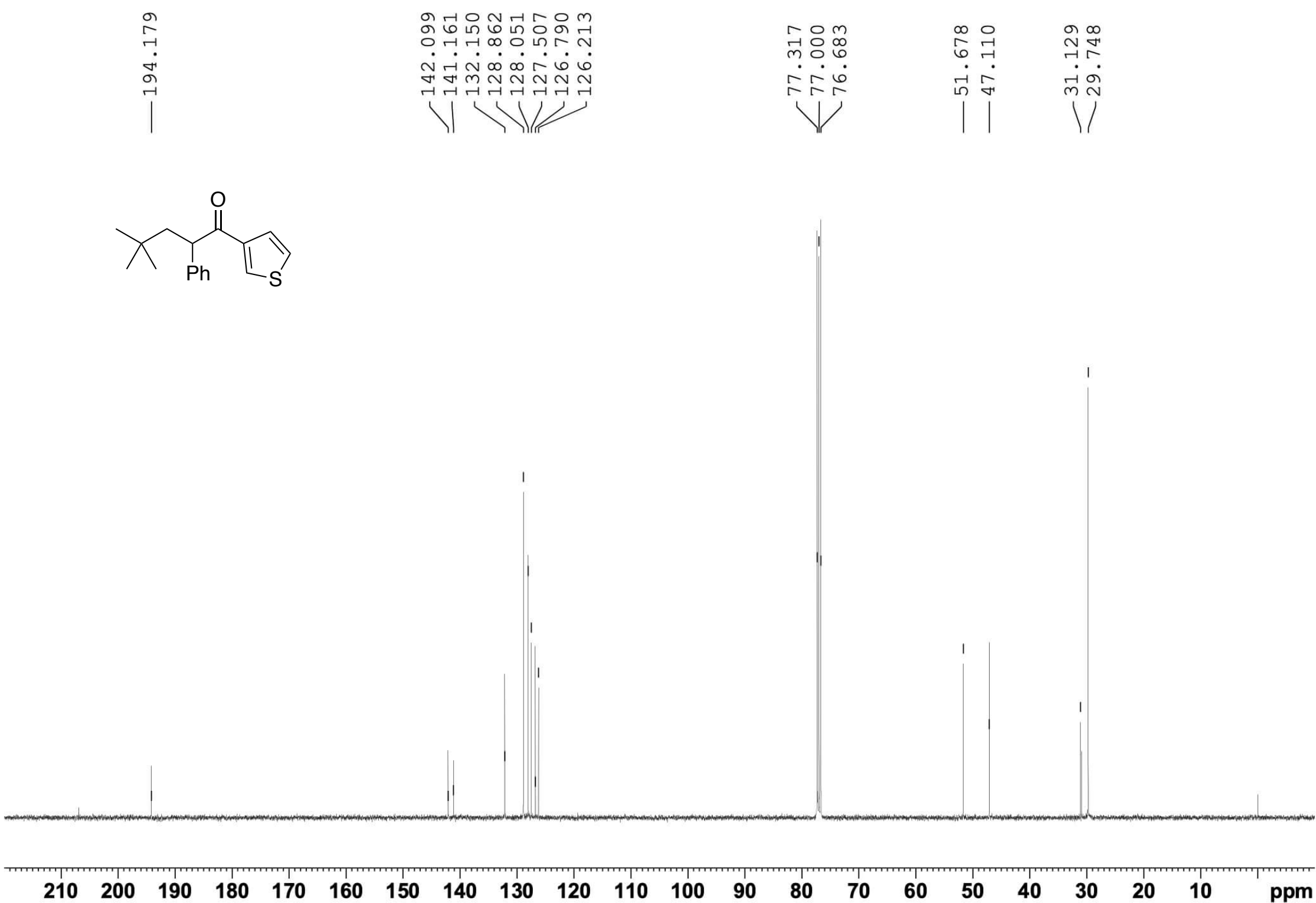

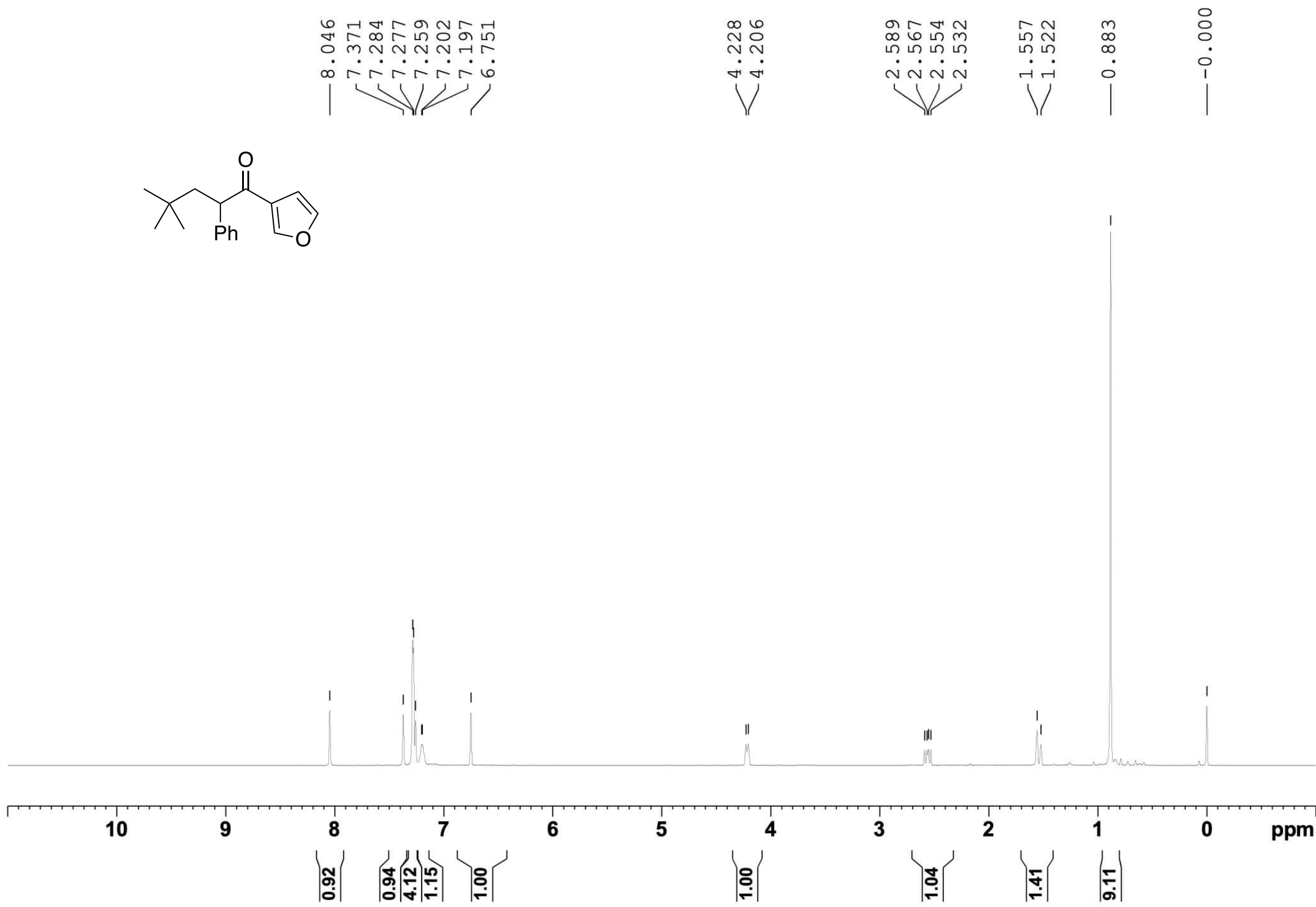


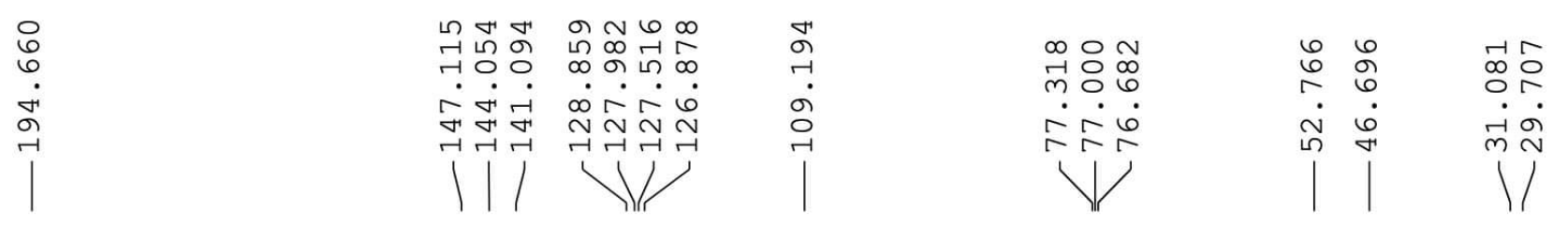
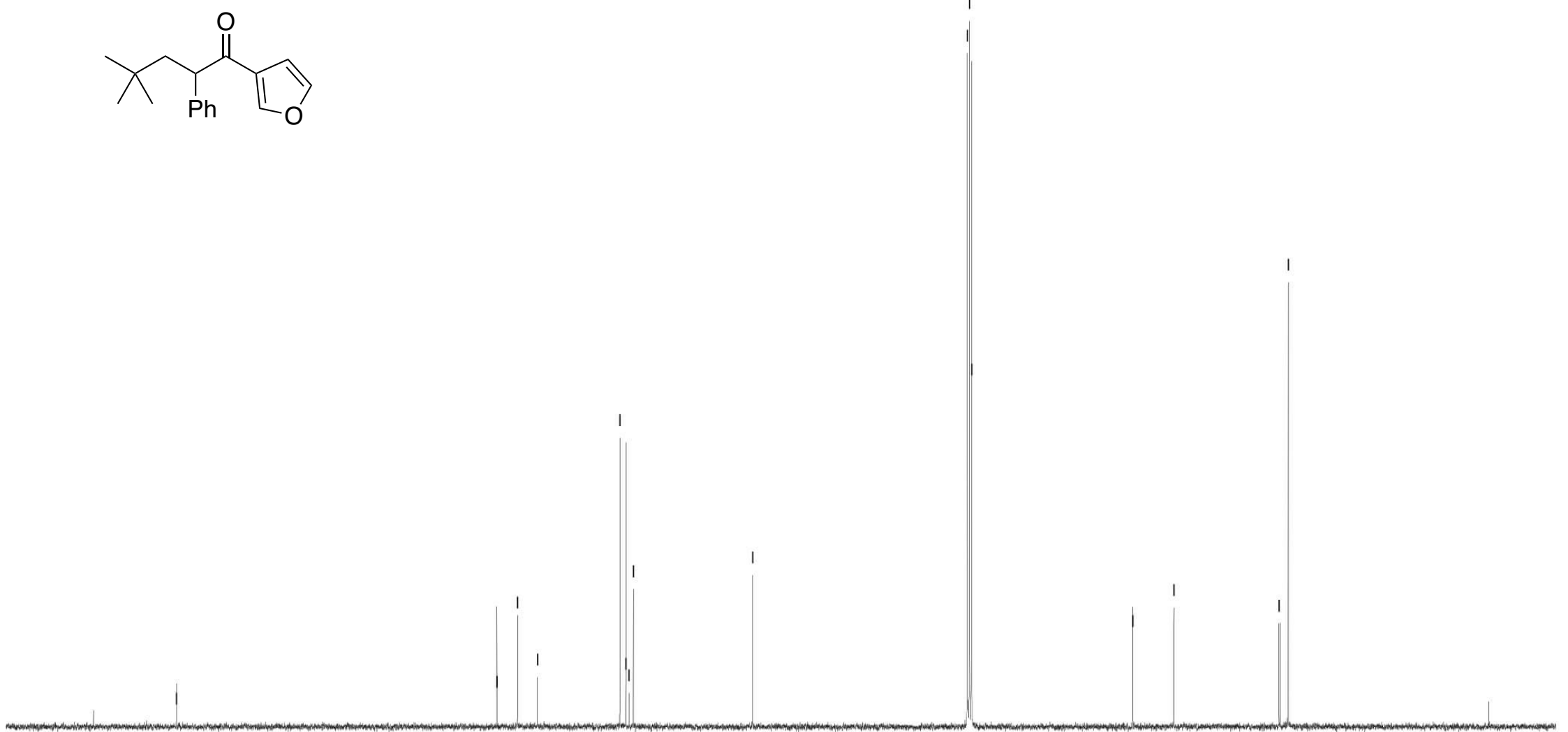

$\begin{array}{llllllllllllllllllllll}210 & 200 & 190 & 180 & 170 & 160 & 150 & 140 & 130 & 120 & 110 & 100 & 90 & 80 & 70 & 60 & 50 & 40 & 30 & 20 & 10 & \text { ppm }\end{array}$ 


\section{${ }^{1} \mathrm{H}$ NMR of 6aai}

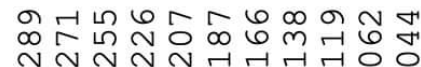

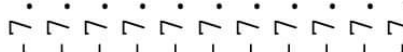

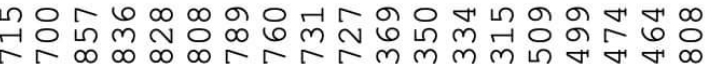

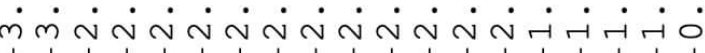

(<smiles>CC(C)(C)CC(C(=O)CCc1ccccc1)c1ccccc1</smiles>

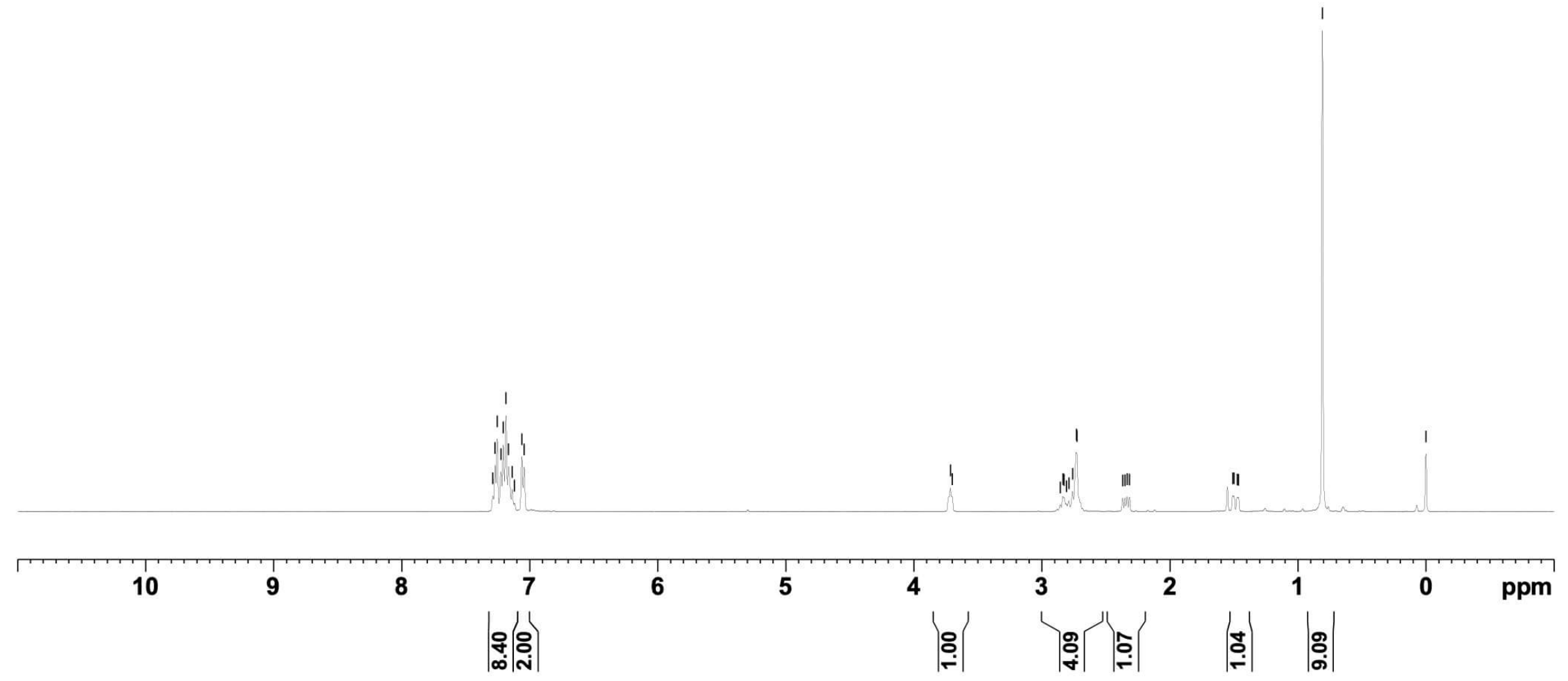



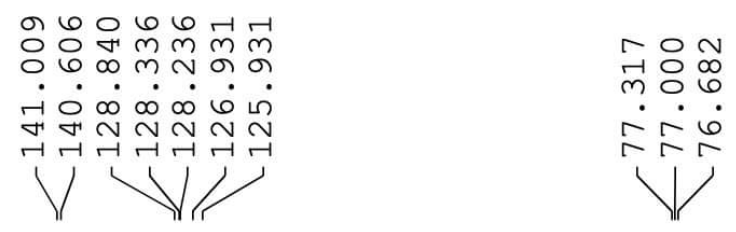

范

ம் ம்ं $\dot{\varnothing} \dot{\sim}$

(n)

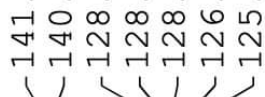<smiles>C[C@@H](CC(C)(C)C)[C@H](C)CCc1ccccc1</smiles>

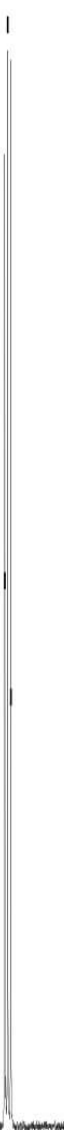

21020019 


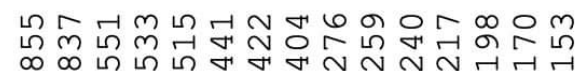

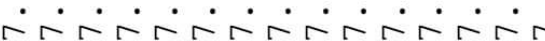

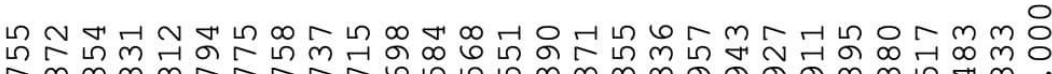

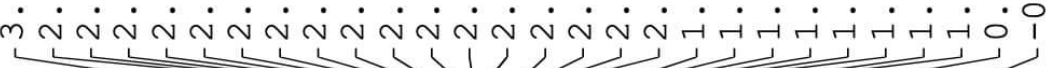<smiles>CC(C)(C)CC(C(=O)CCCC(=O)c1ccccc1)c1ccccc1</smiles>

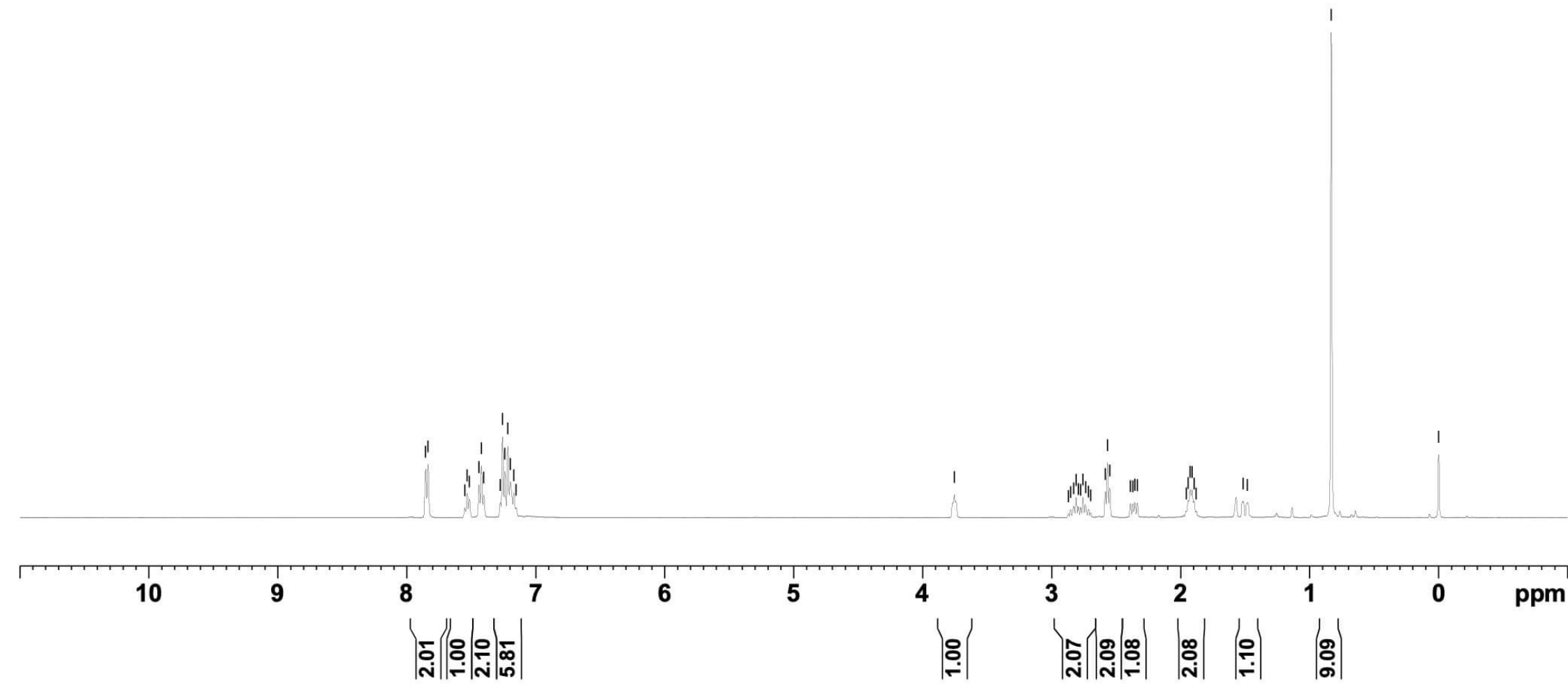



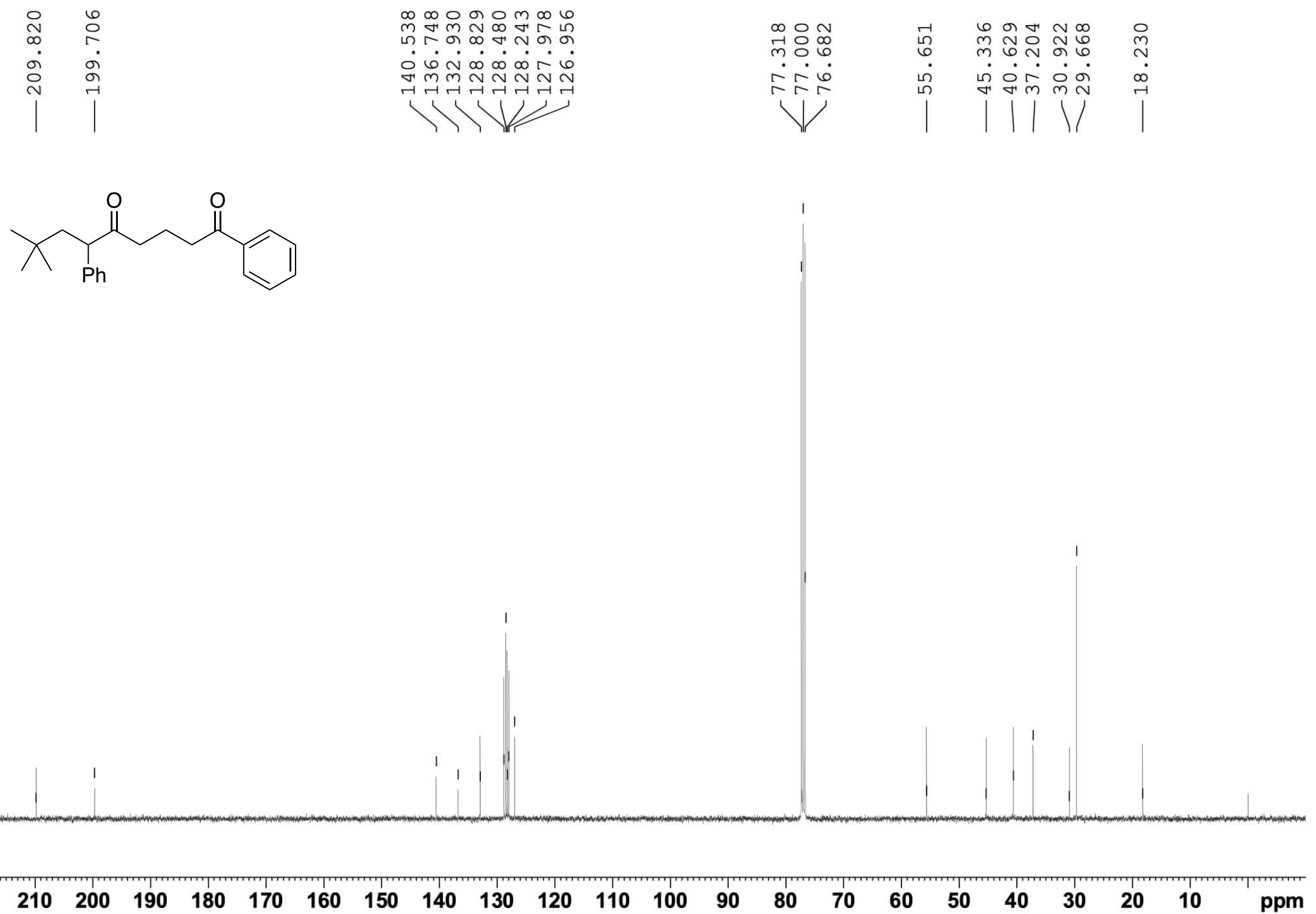


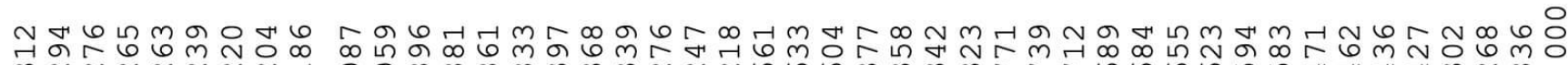

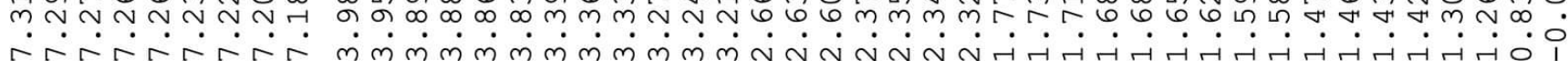
수숫
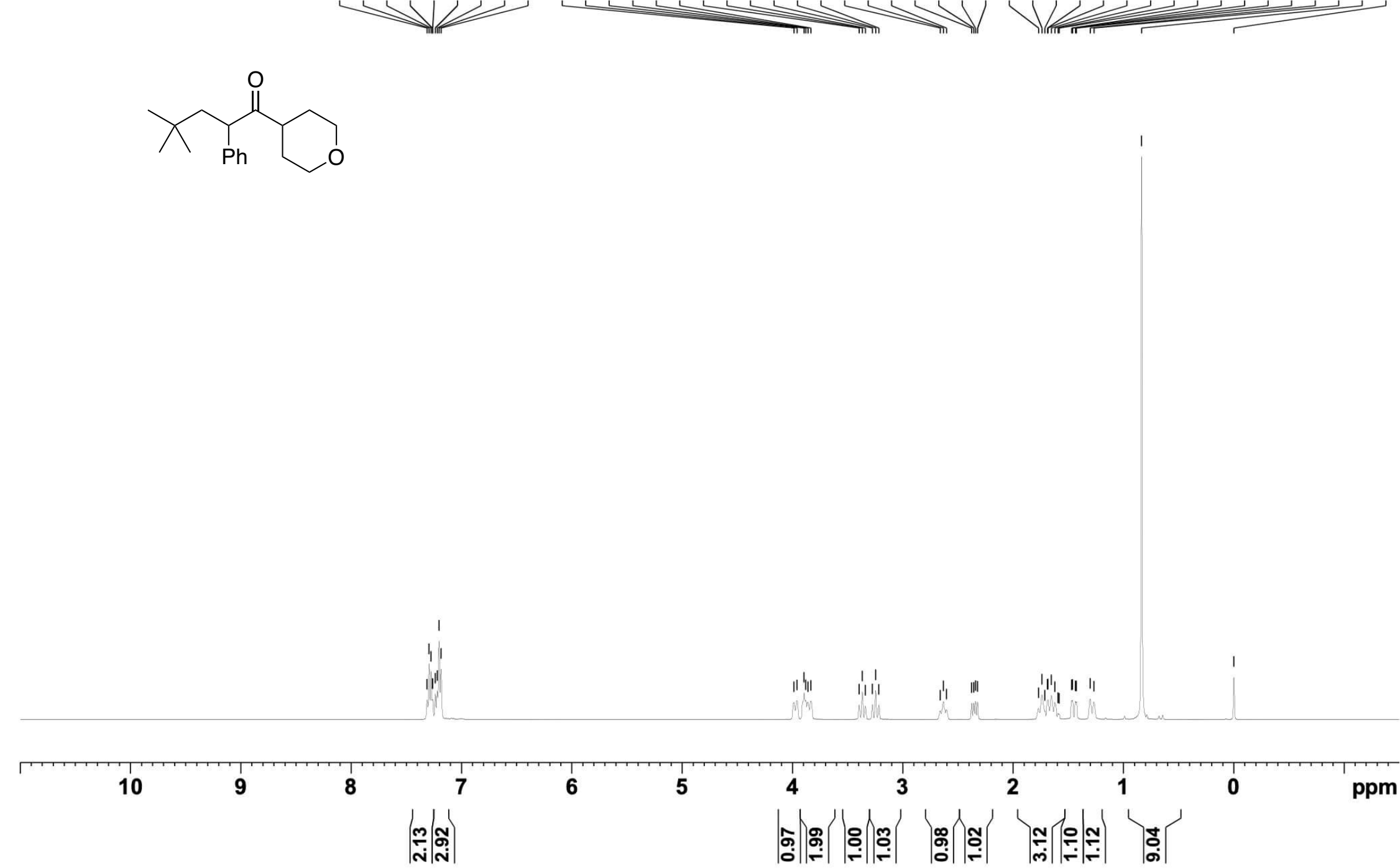

(1) 
$\dot{0}$

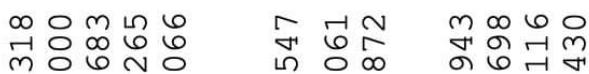

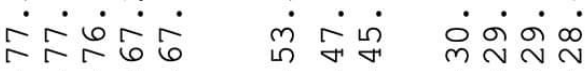

W $1 / 1 /$
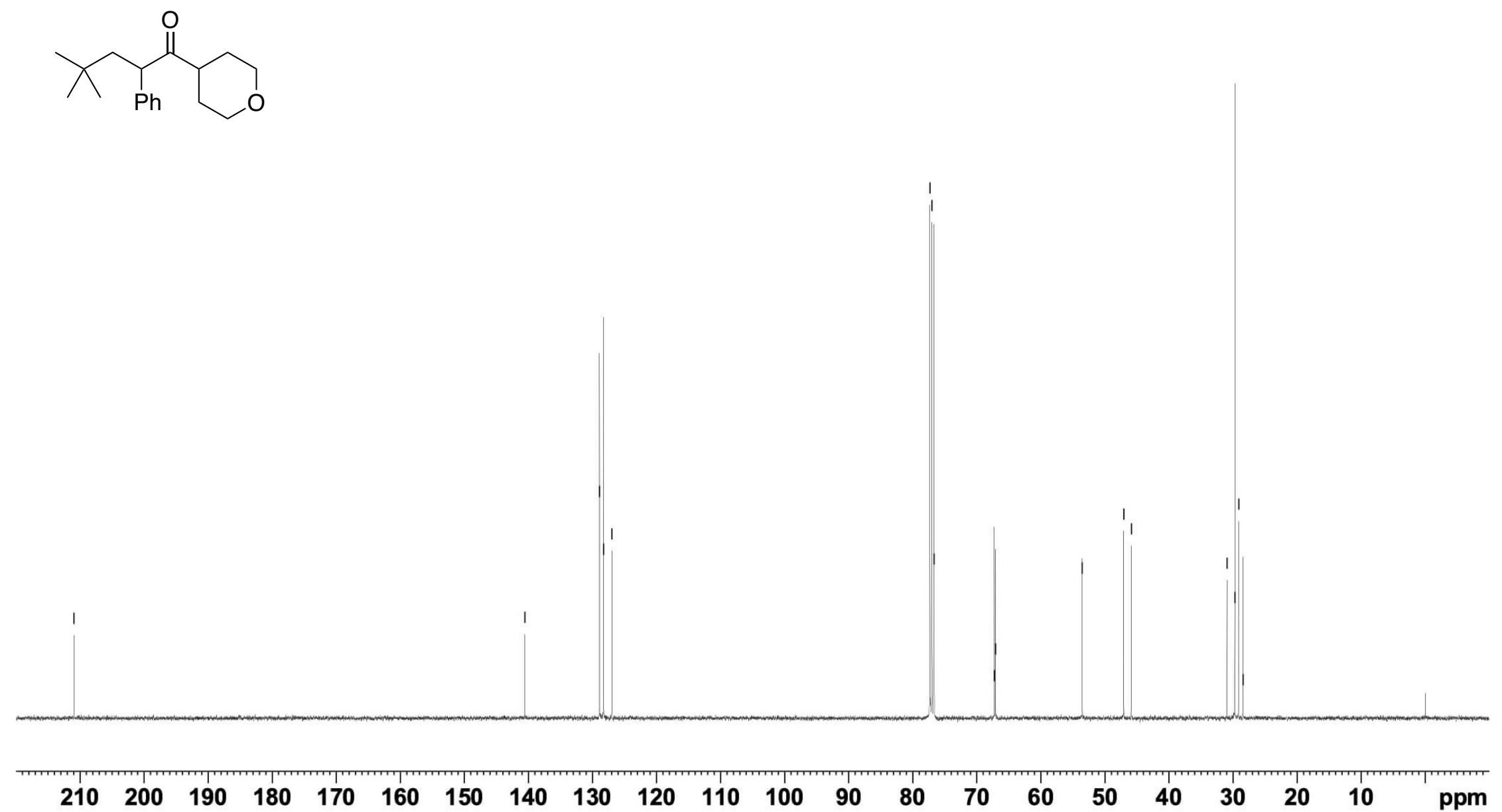


\section{${ }^{1} \mathrm{H}$ NMR of 6aas}

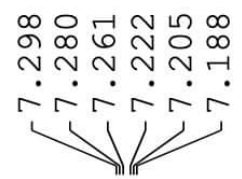

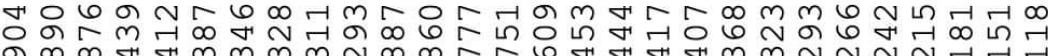
$\dot{m} \dot{m} \dot{\sim} \dot{\sim} \dot{\sim} \dot{\sim} \dot{\sim} \dot{-} \dot{-} \dot{-} \dot{-} \dot{-} \cdot \dot{-} \cdot \dot{-} \dot{-}$

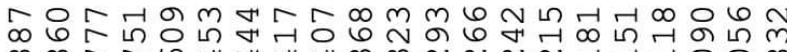

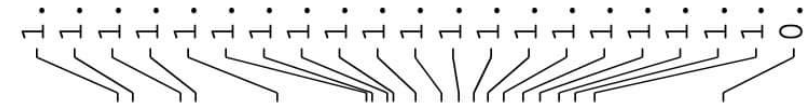

웅
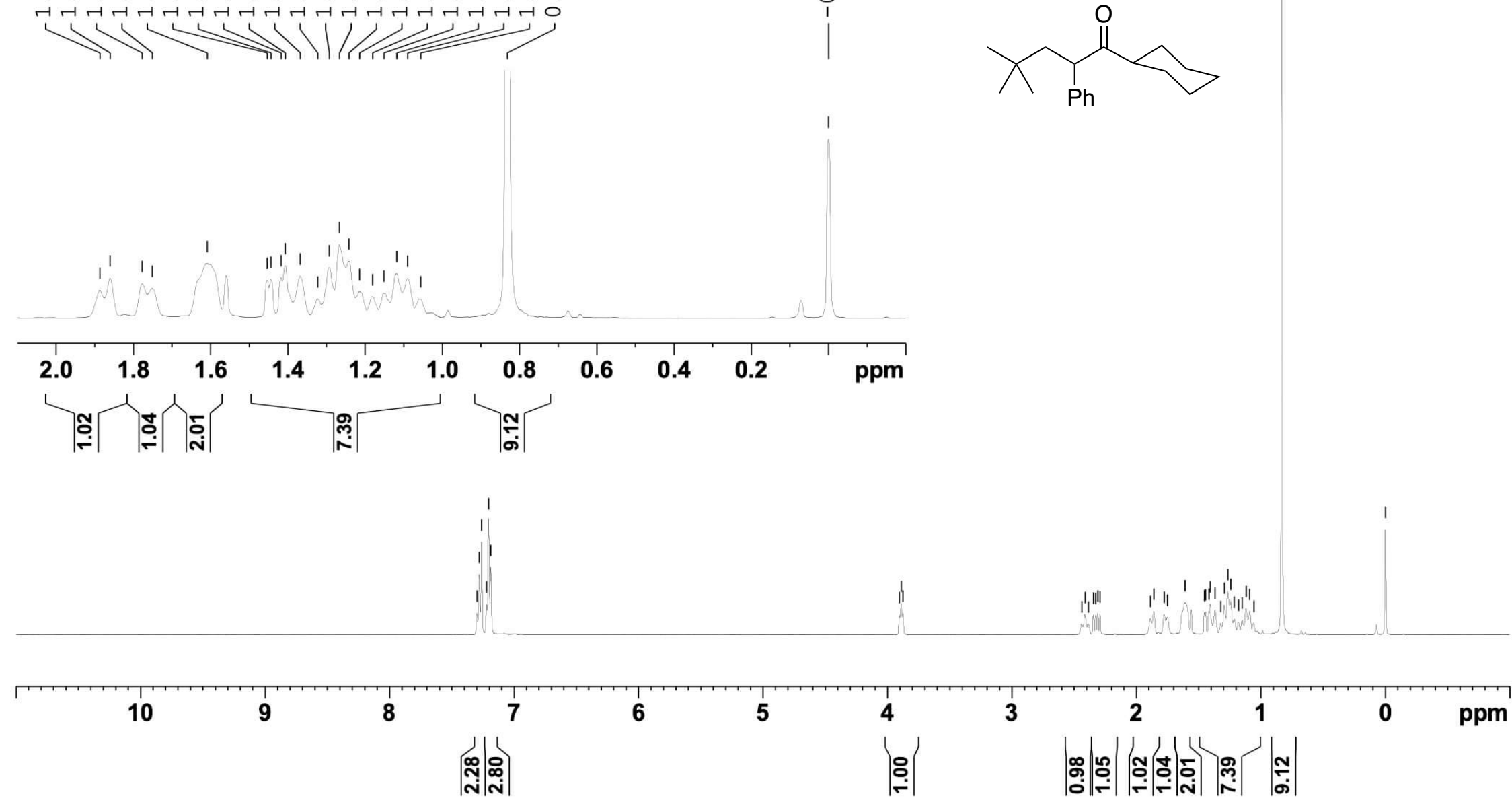

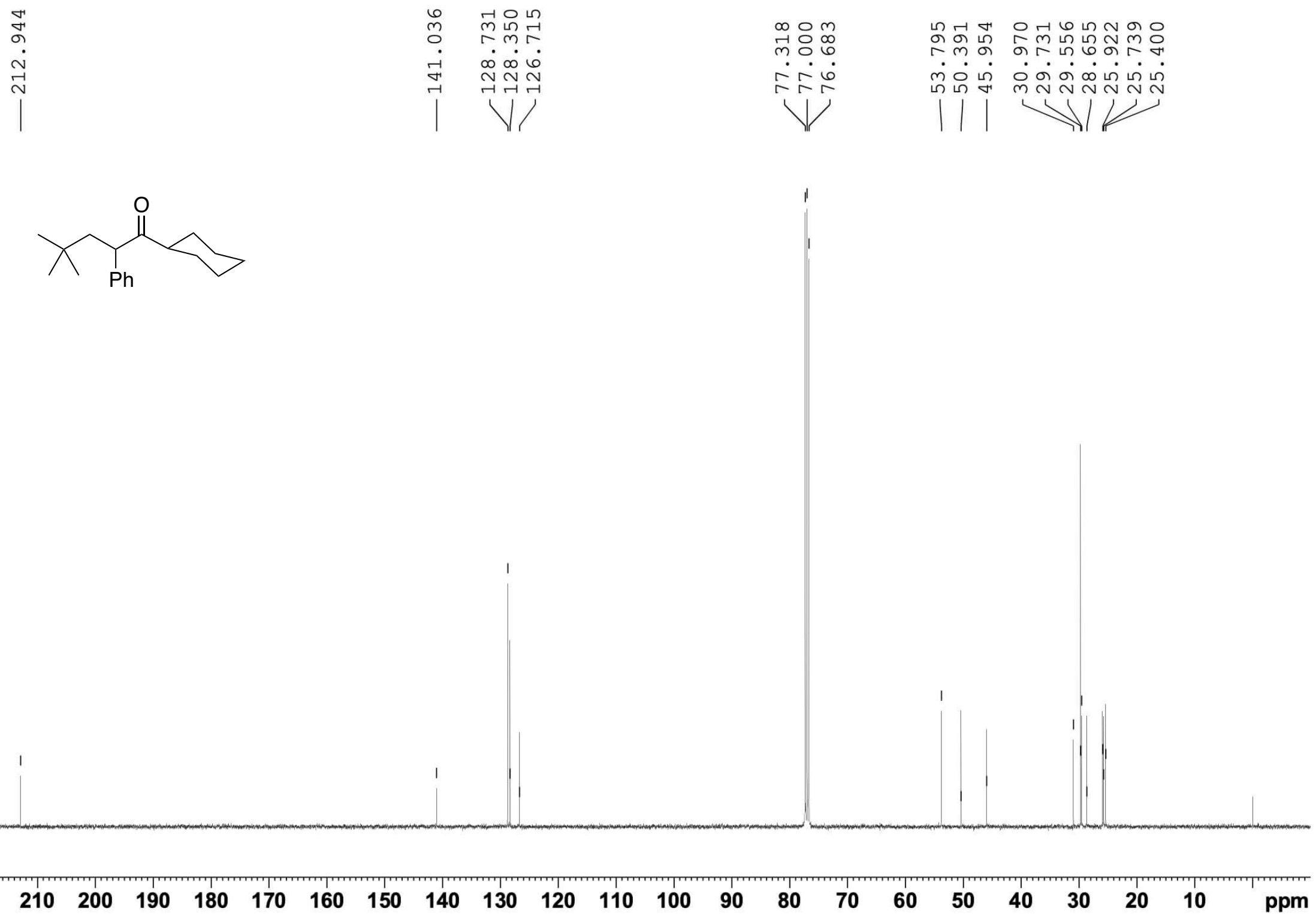


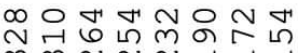

irisiris

1

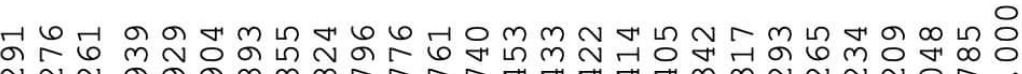
सं y
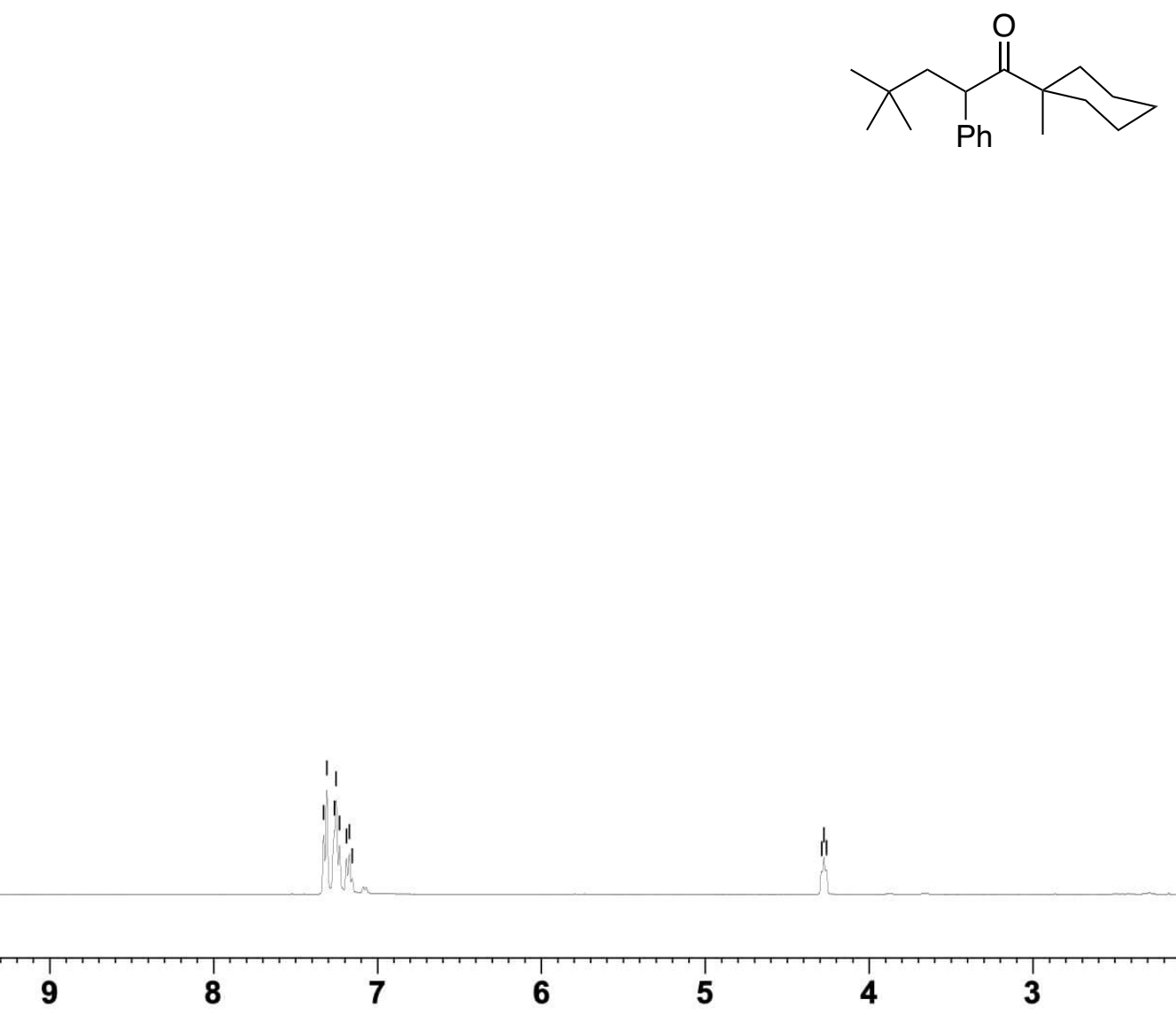

9

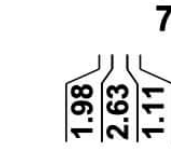

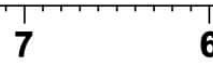

6

5

|

2

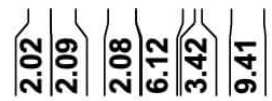



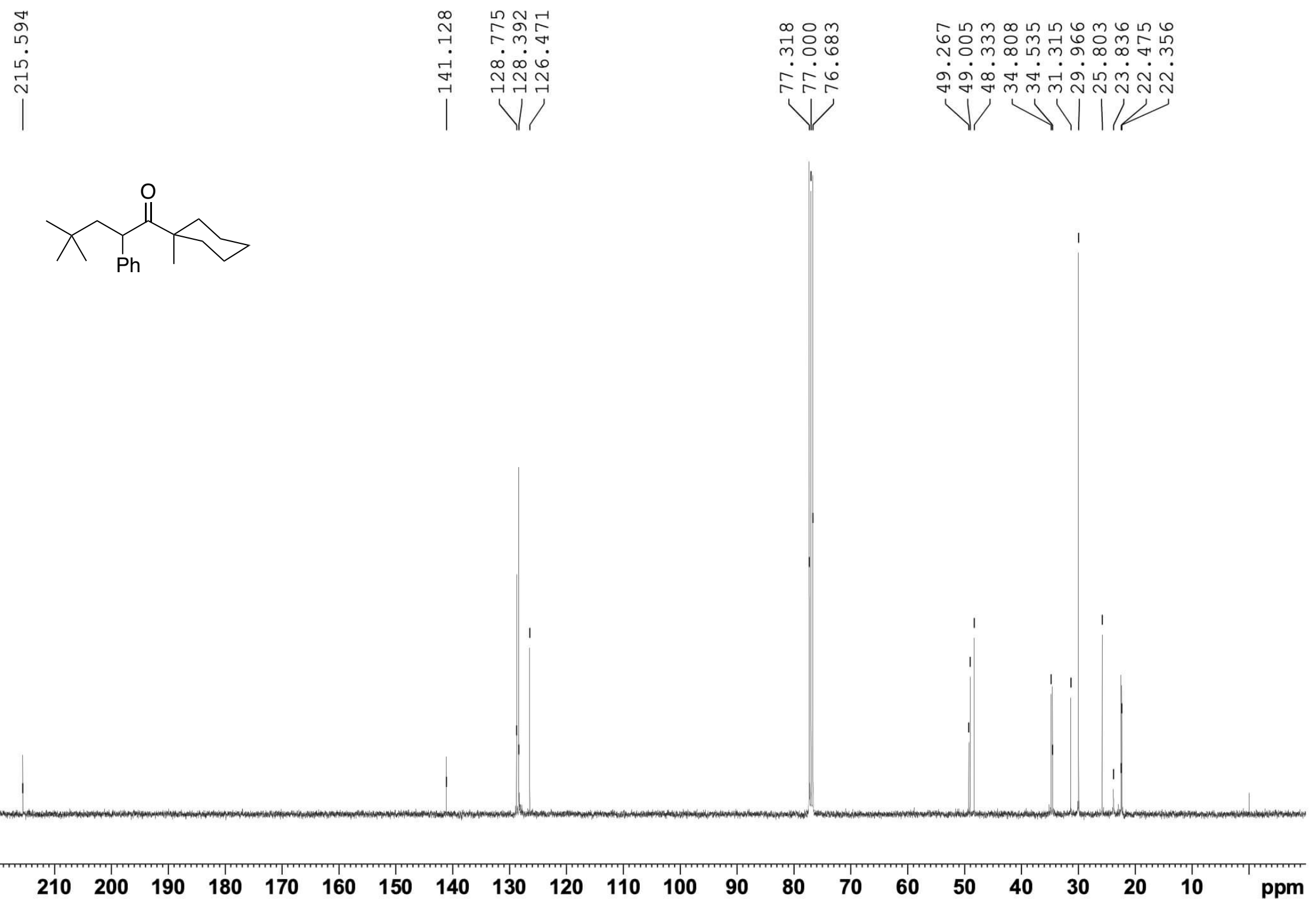


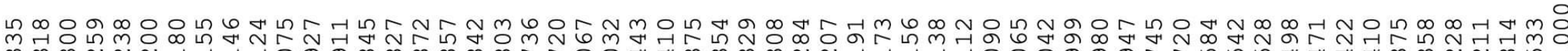

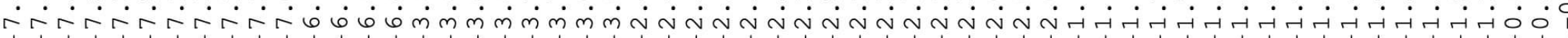
Tा111111 1 กा
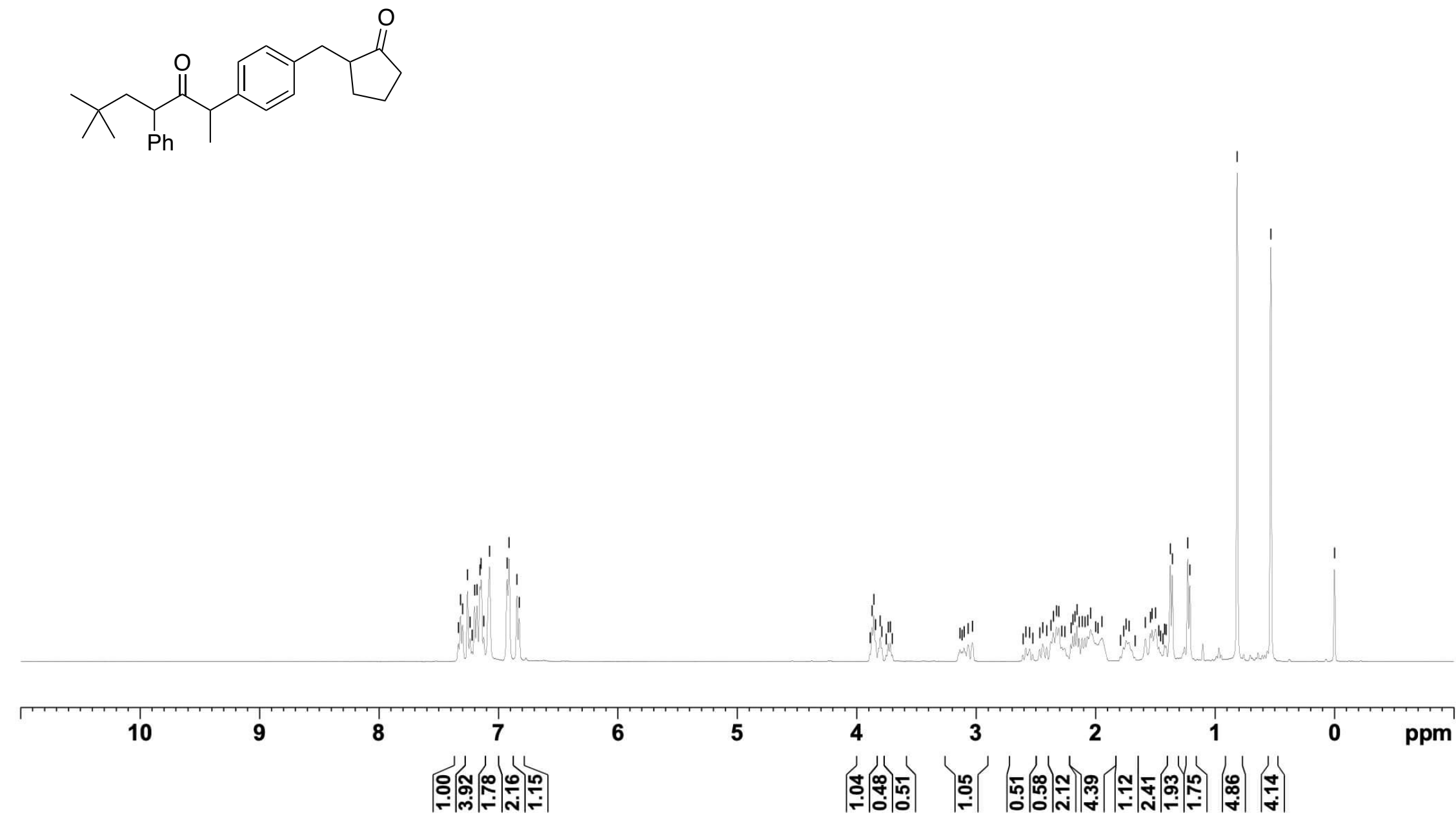


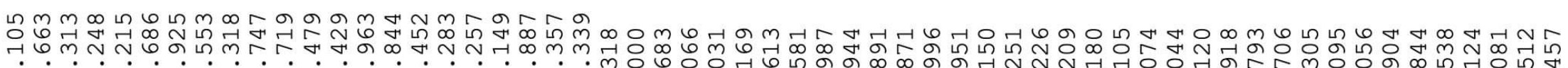

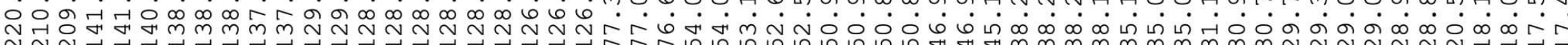
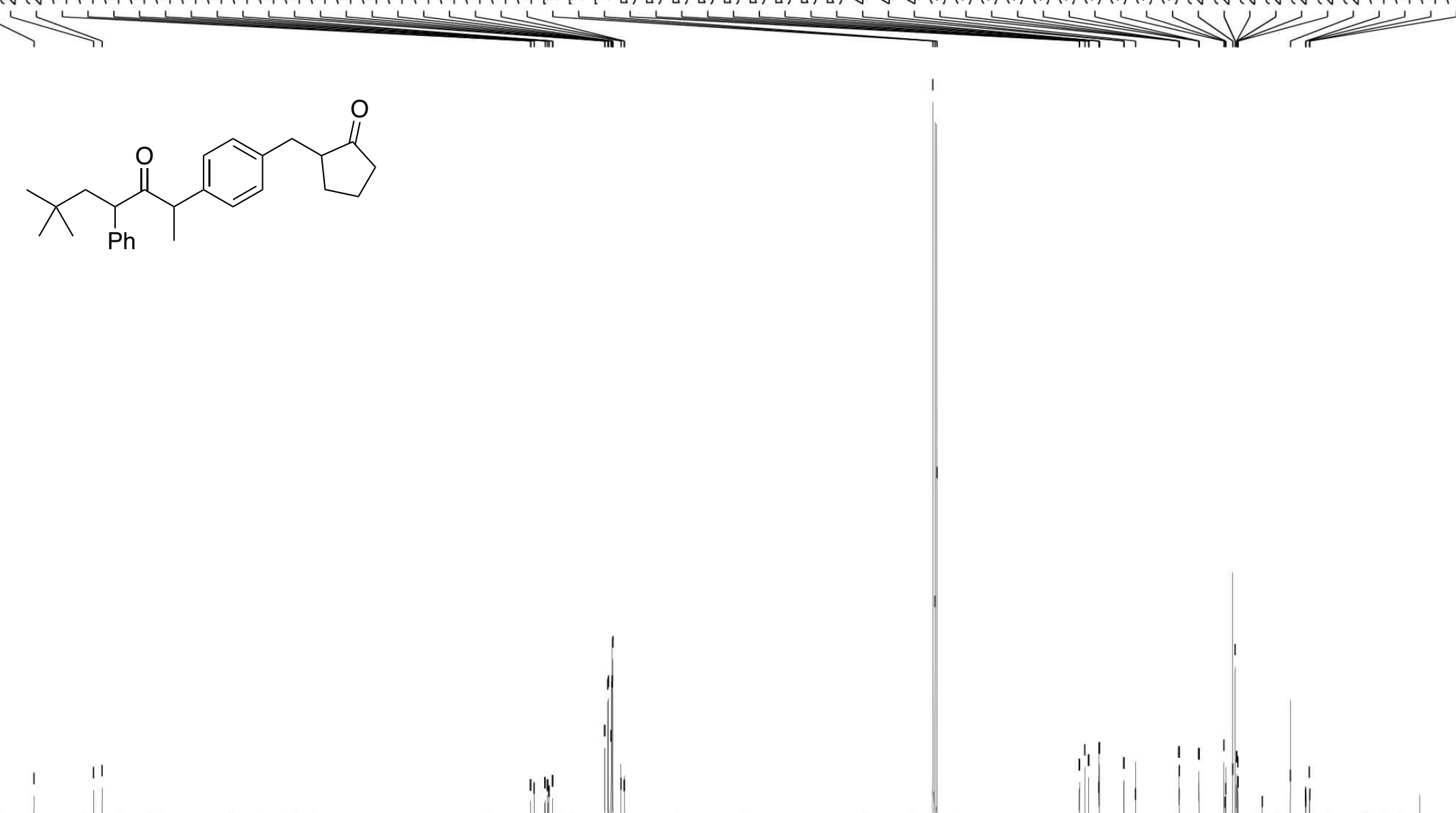

$50140130120110100 \quad 90$ 


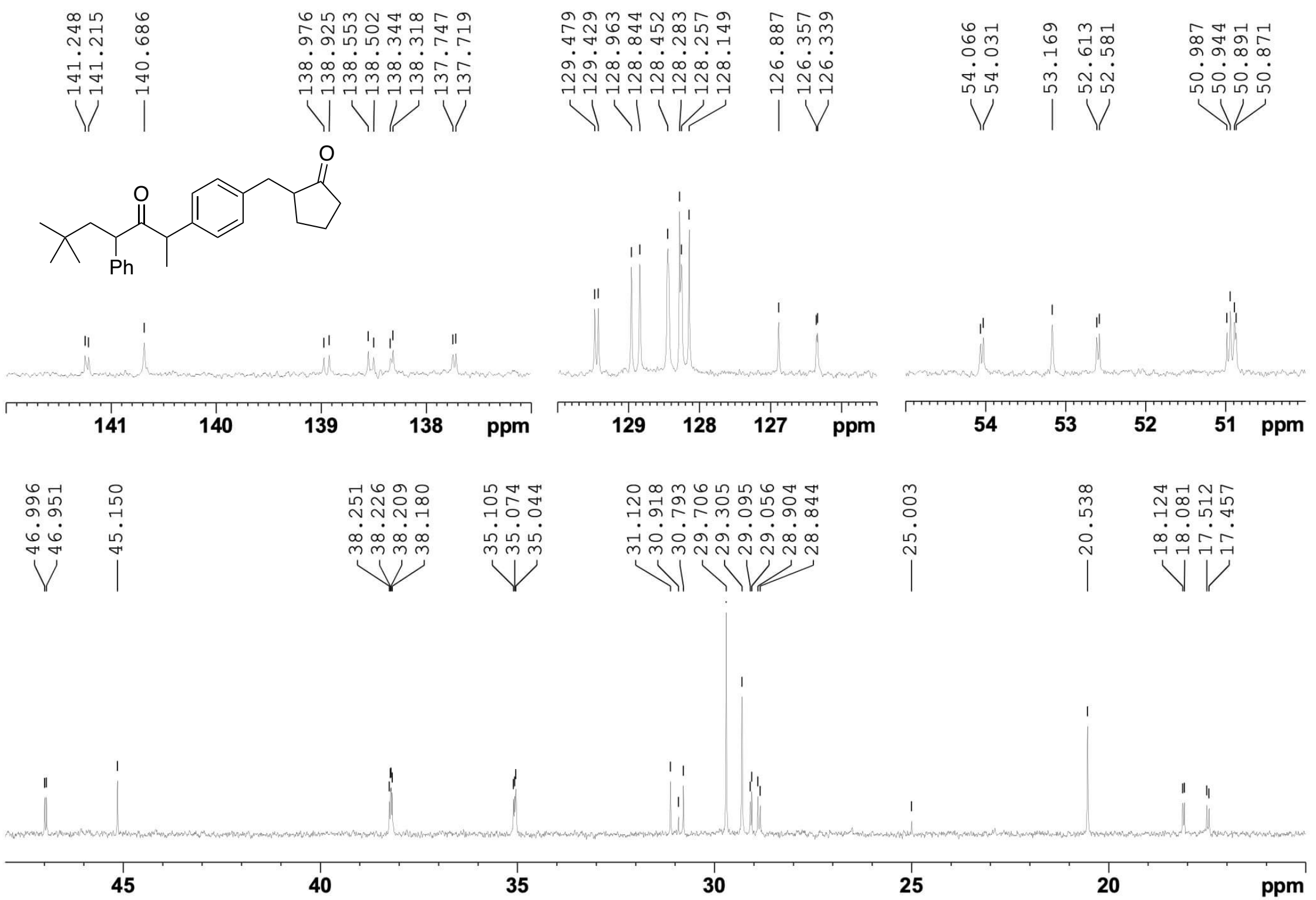




\section{${ }^{1} \mathrm{H}$ NMR of $\mathbf{6 a a v}$}
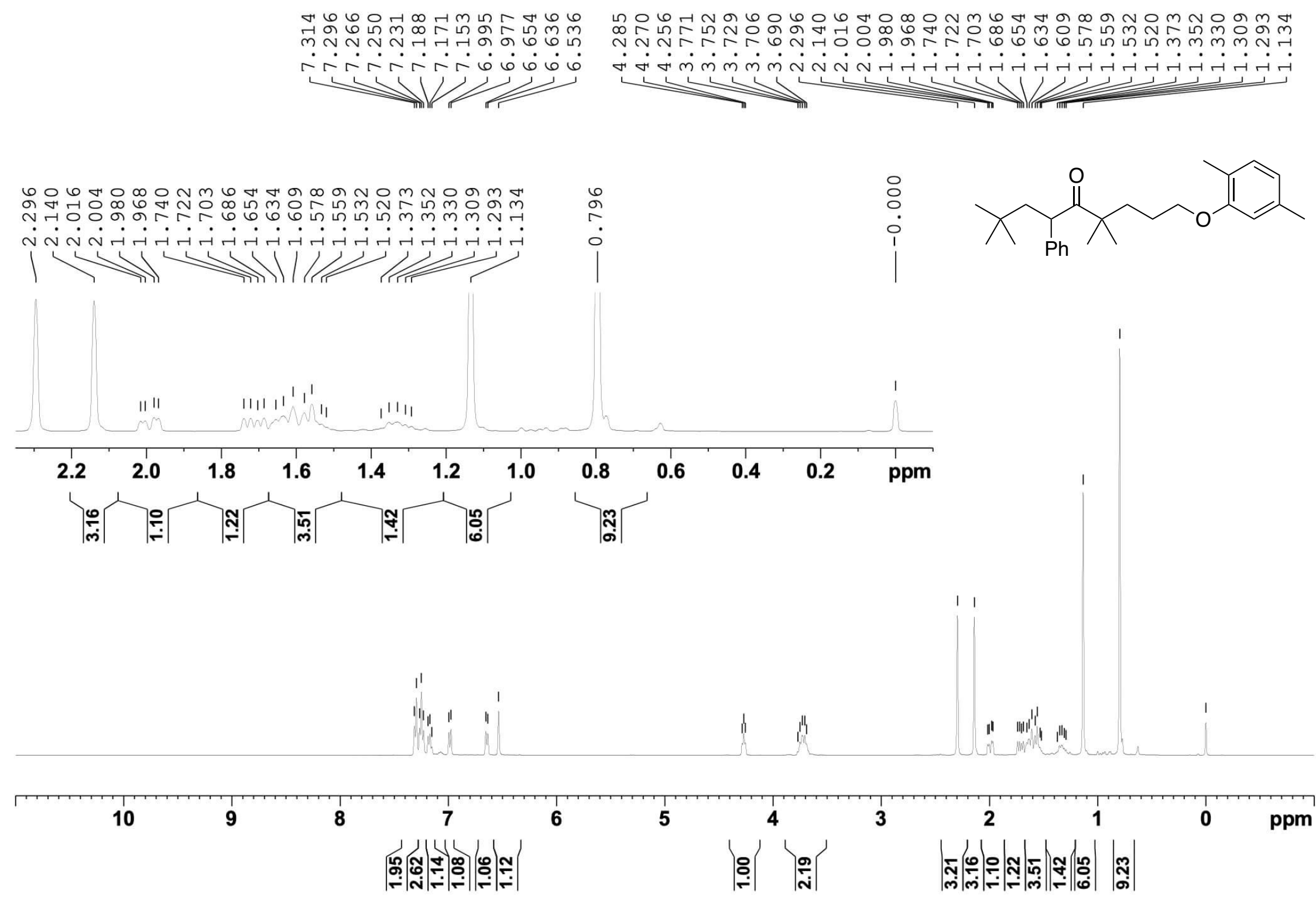

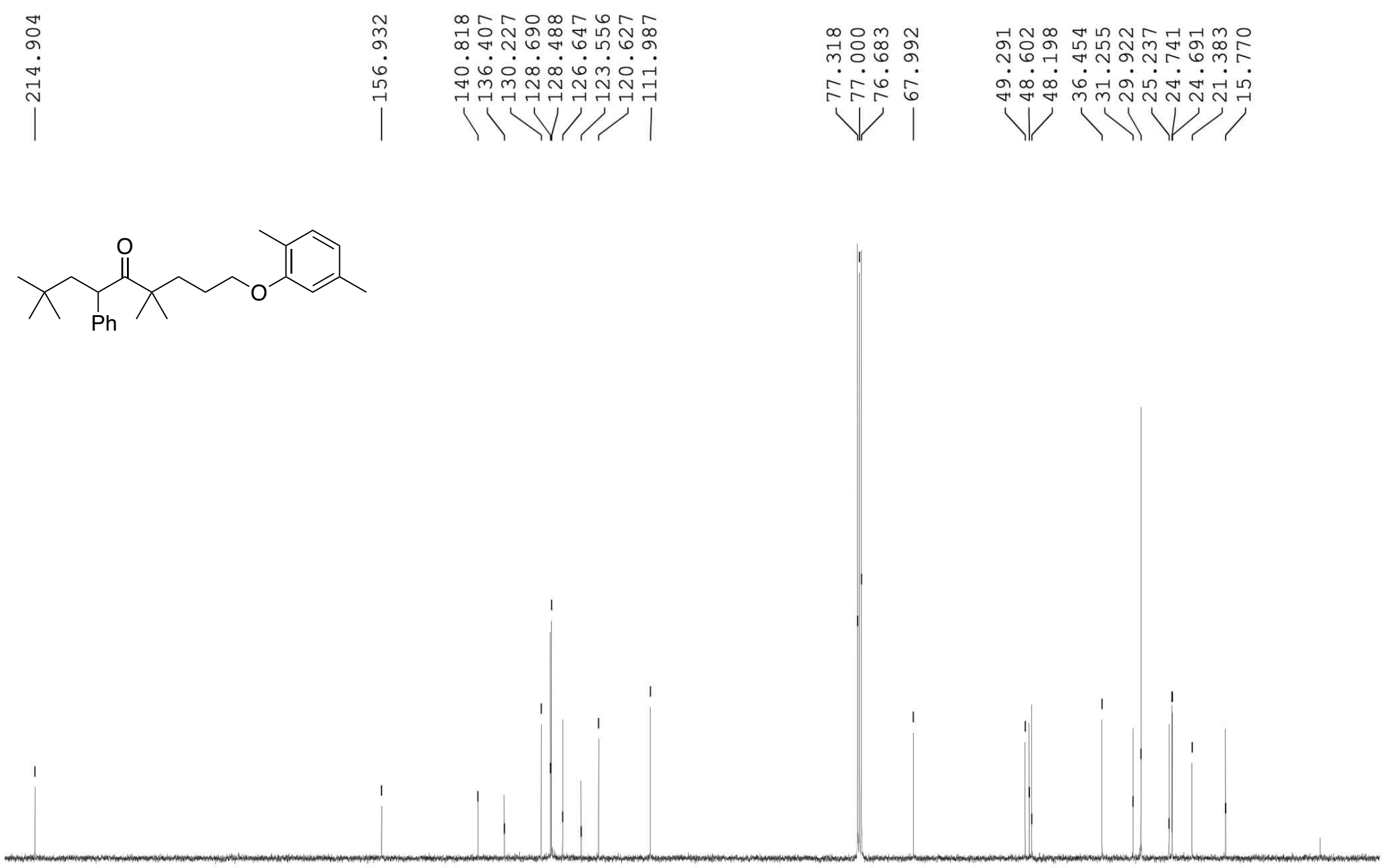

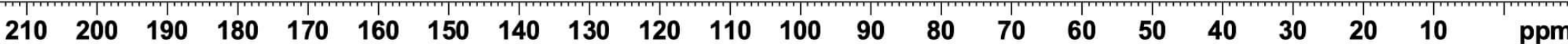


${ }^{1} \mathrm{H}$ NMR of $\mathbf{6 a b a}$

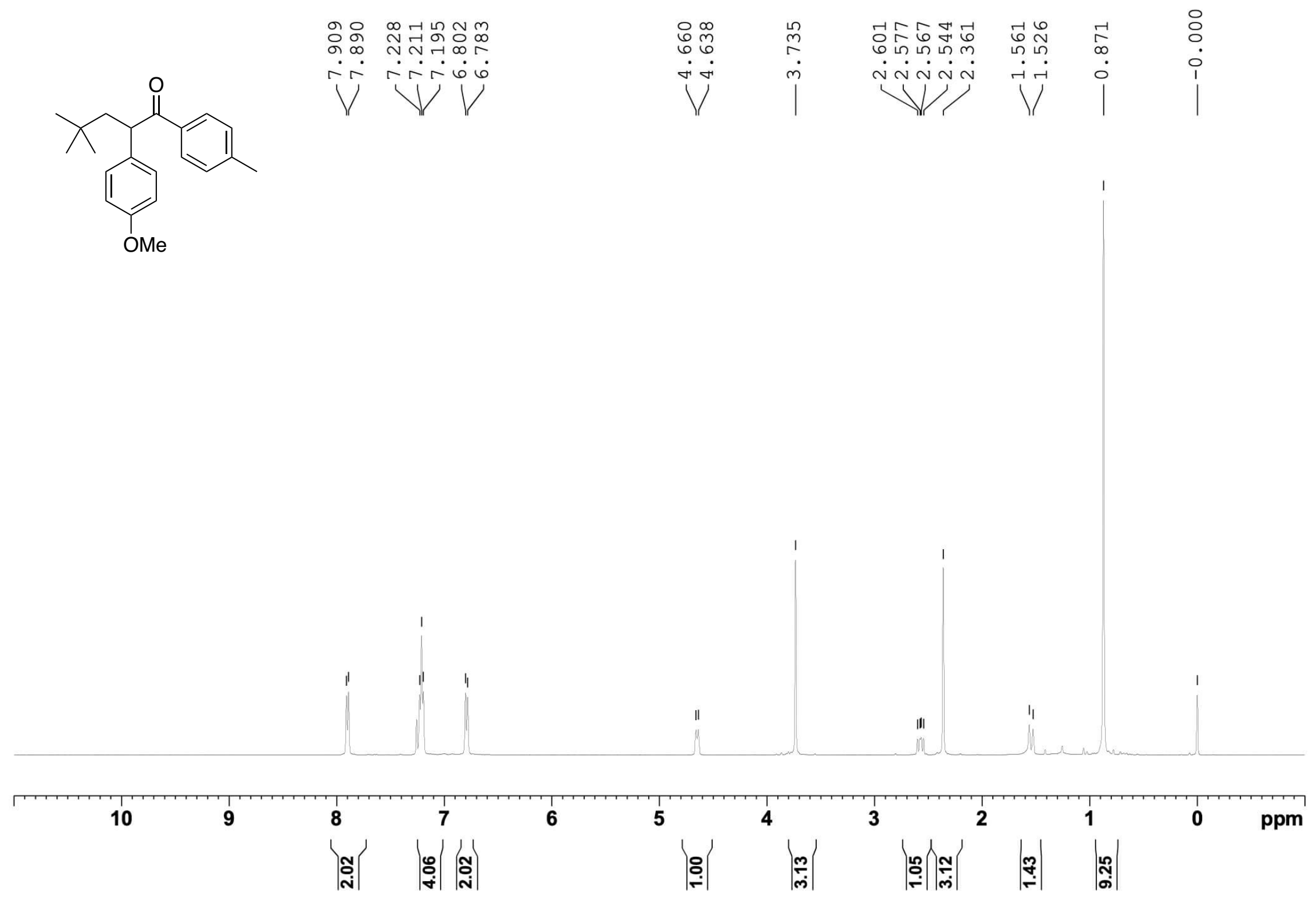




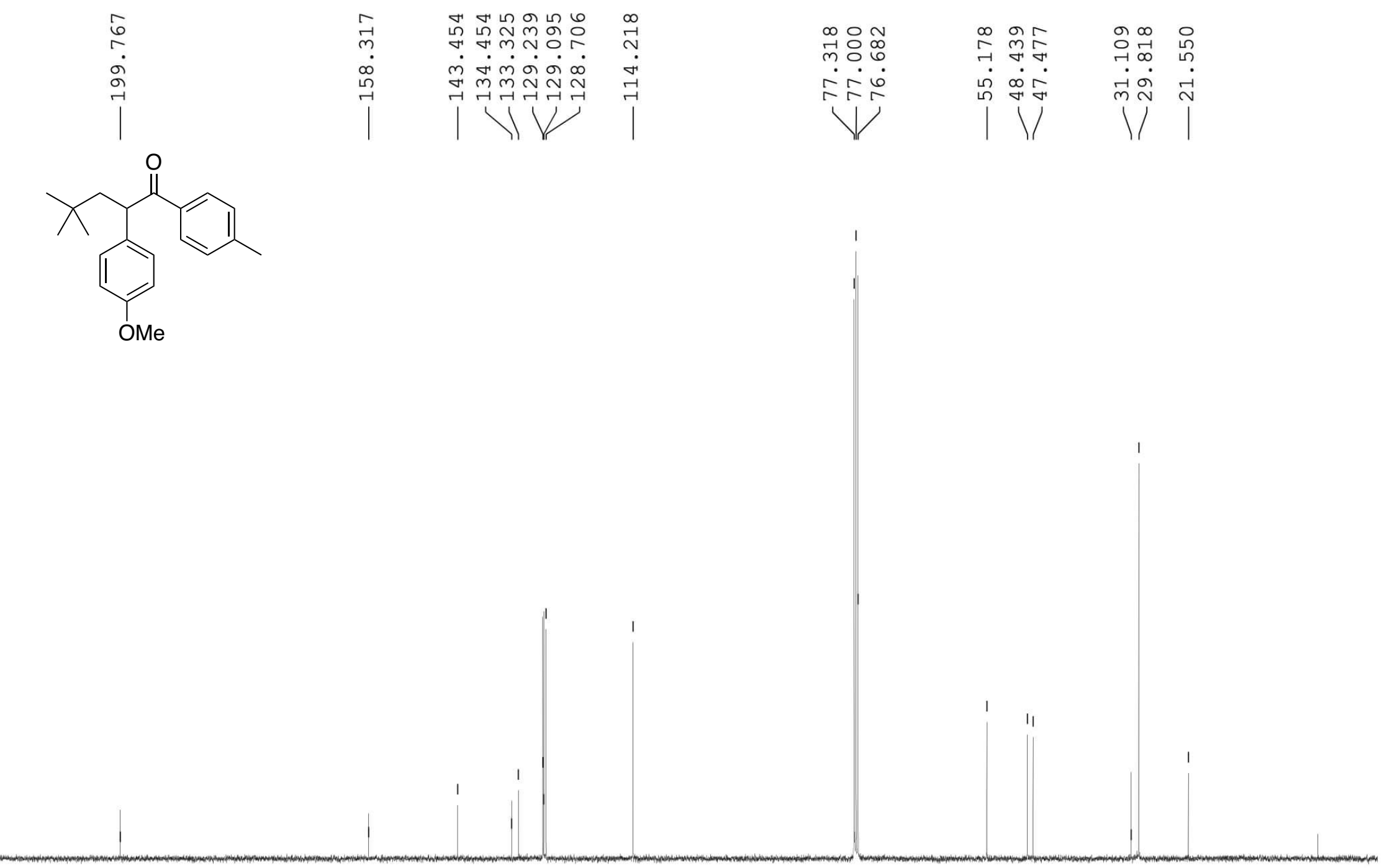

$\begin{array}{llllllllllll}210 & 200 & 190 & 180 & 170 & 160 & 150 & 140 & 130 & 120 & 110 & 100\end{array}$

$\begin{array}{lllllll}50 & 40 & 30 & 20 & 10 & \mathrm{ppm}\end{array}$ 


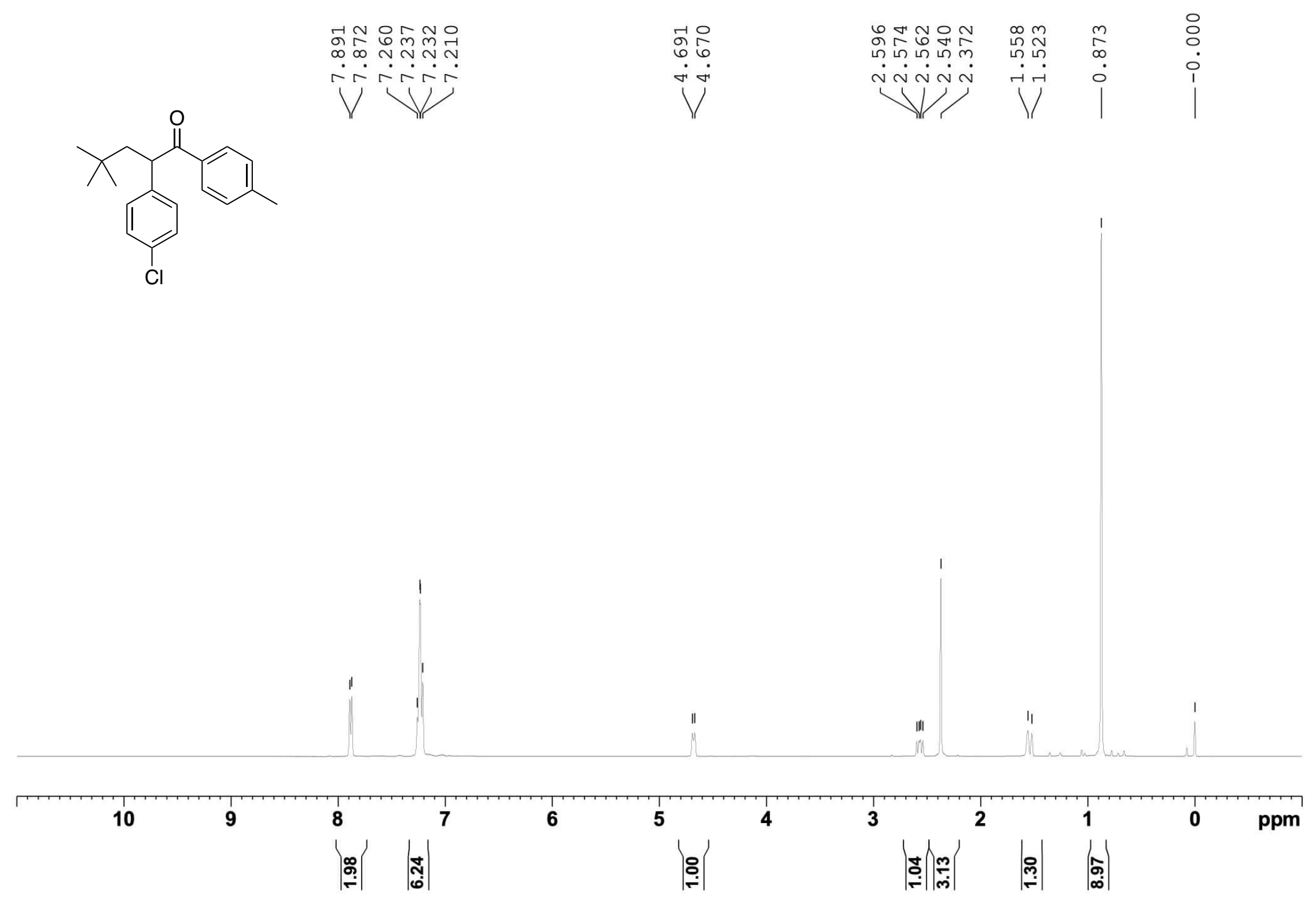


${ }^{13} \mathrm{C}$ NMR of 6aca
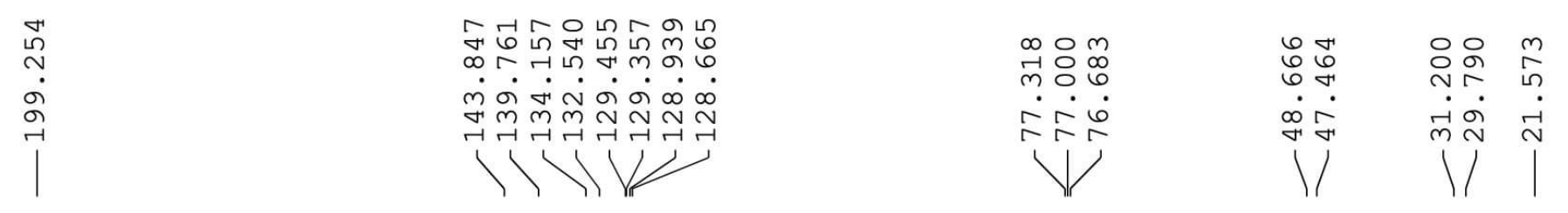<smiles>Cc1ccc(C(=O)C(CC(C)(C)C)c2ccc(Cl)cc2)cc1</smiles>
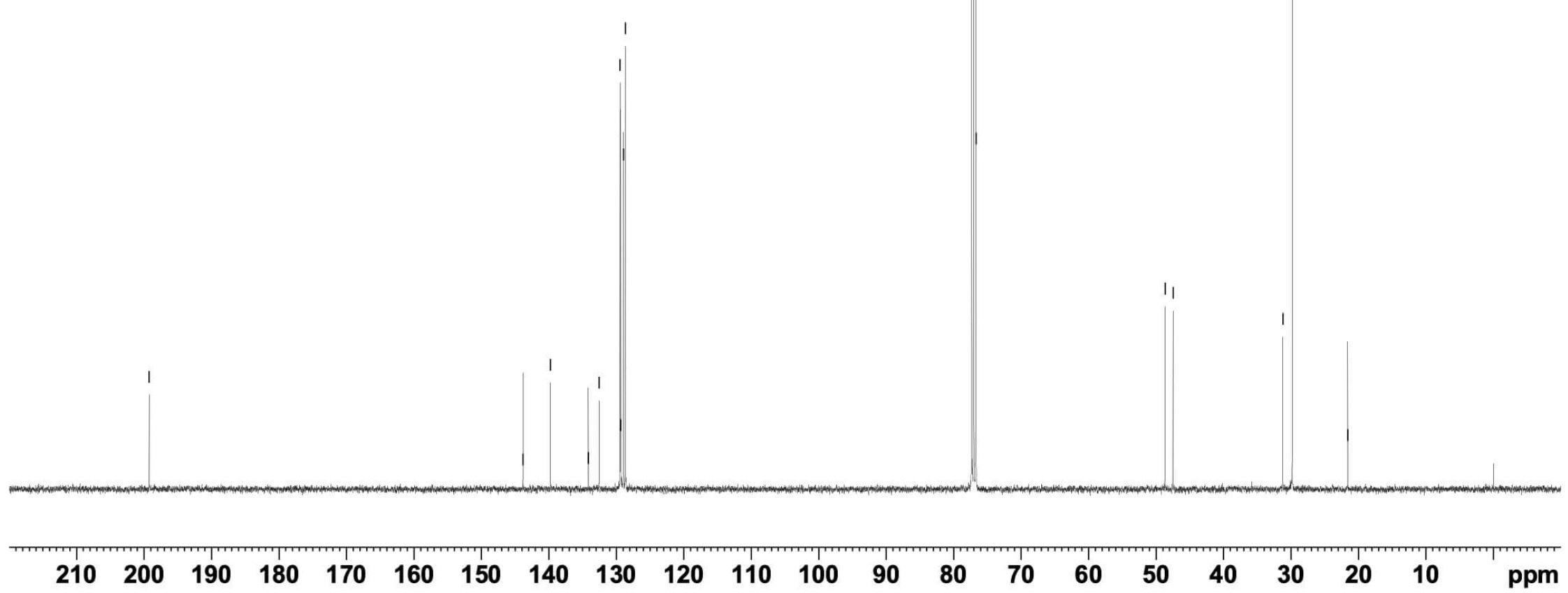

S155 

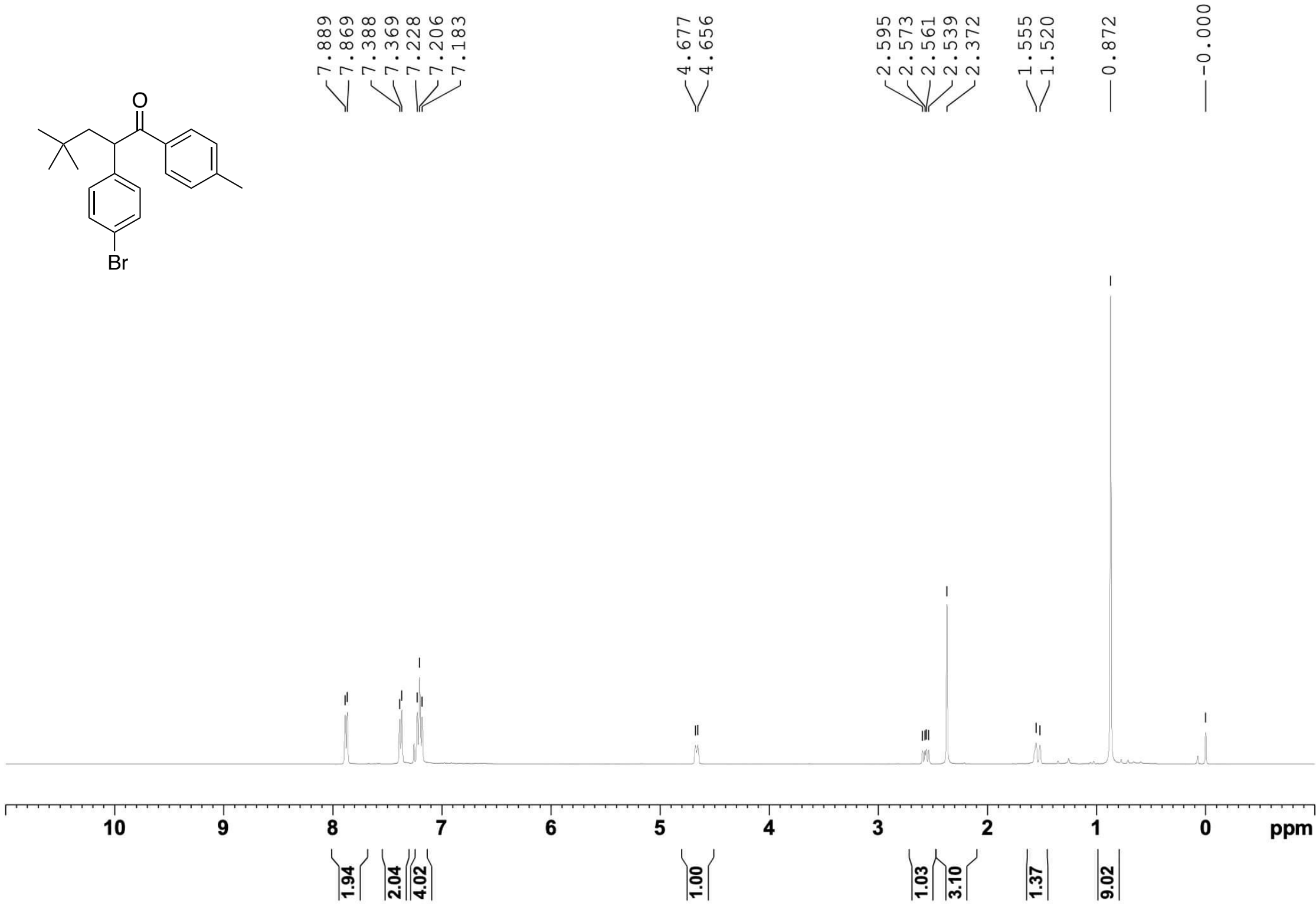

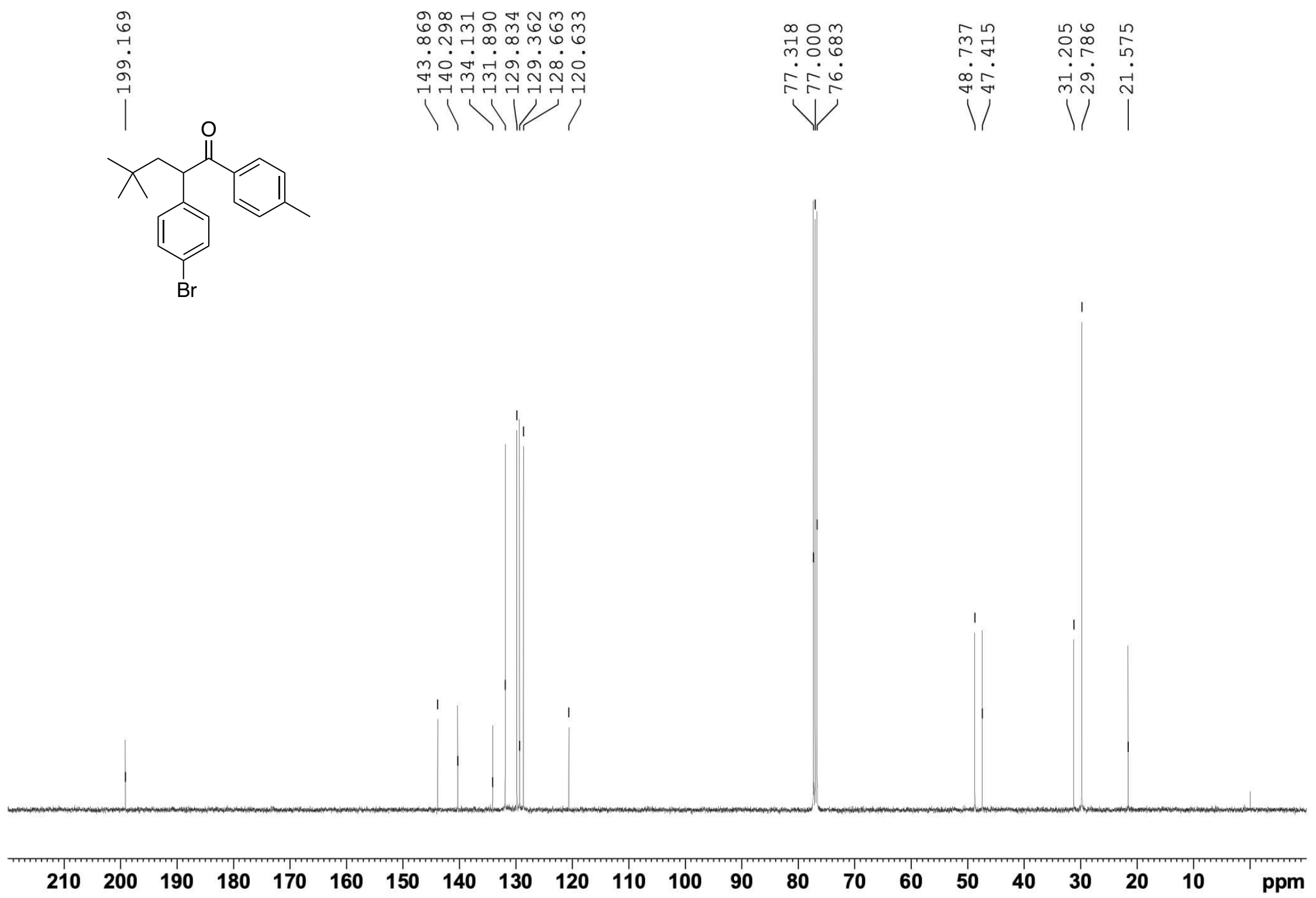


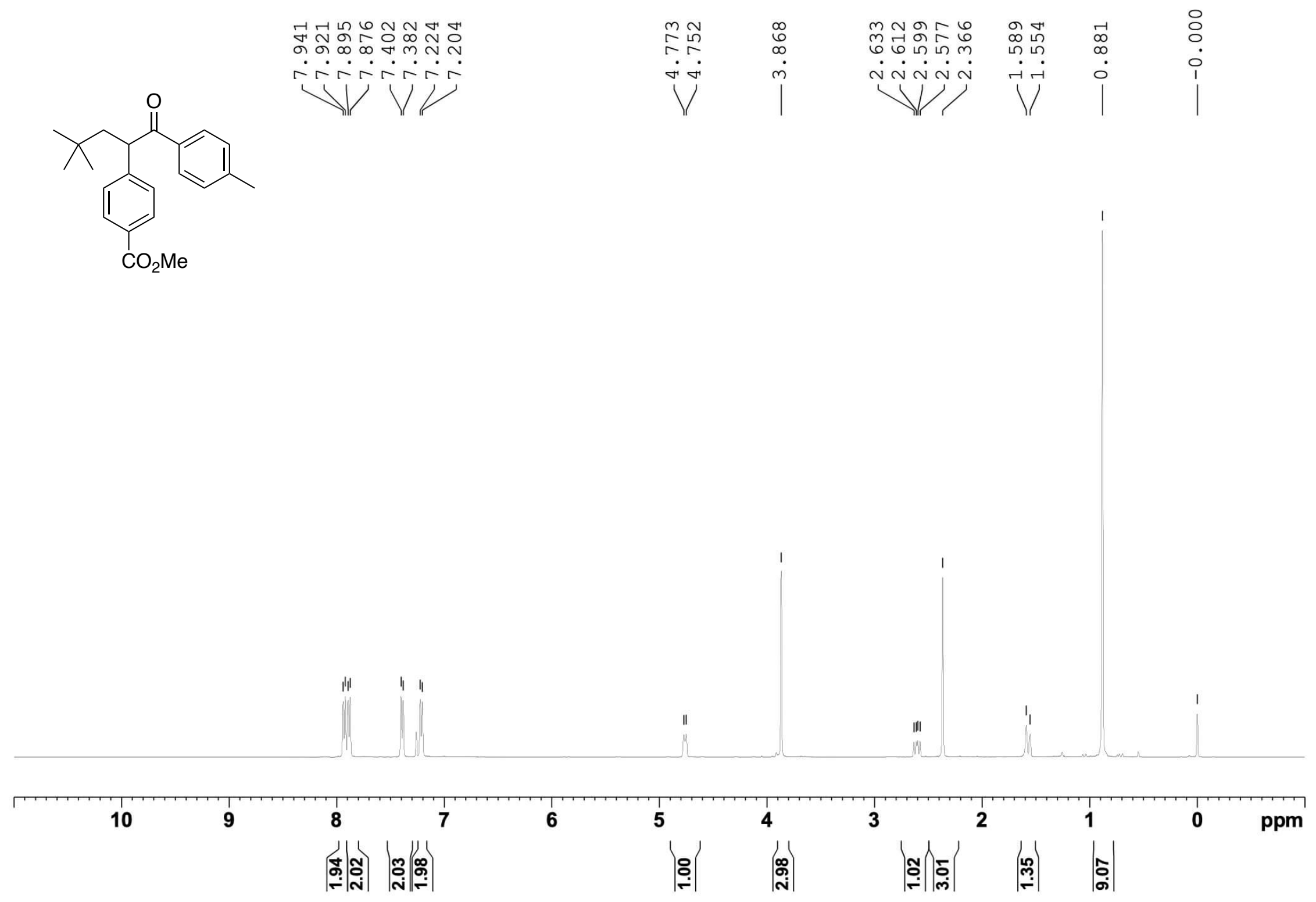



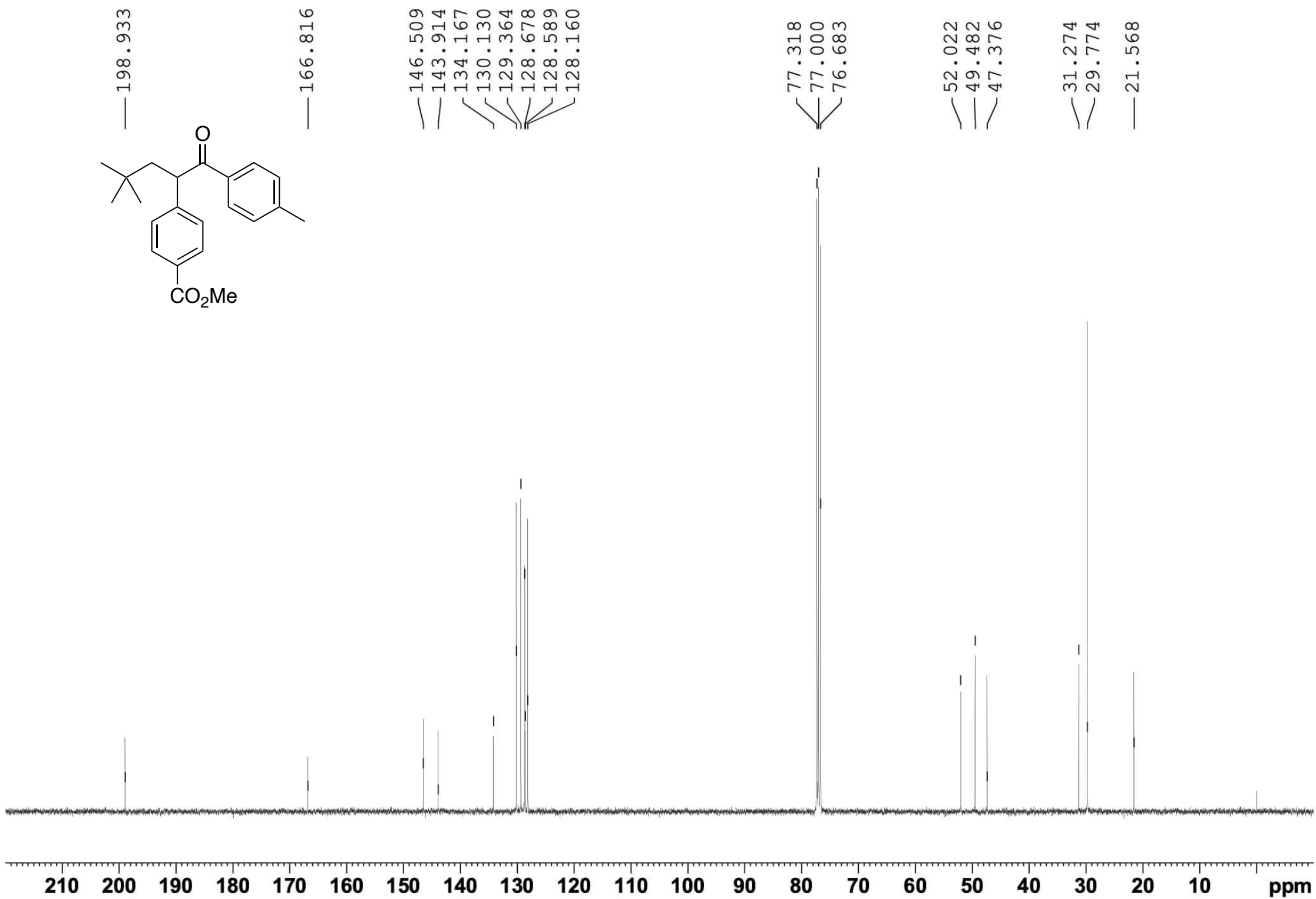


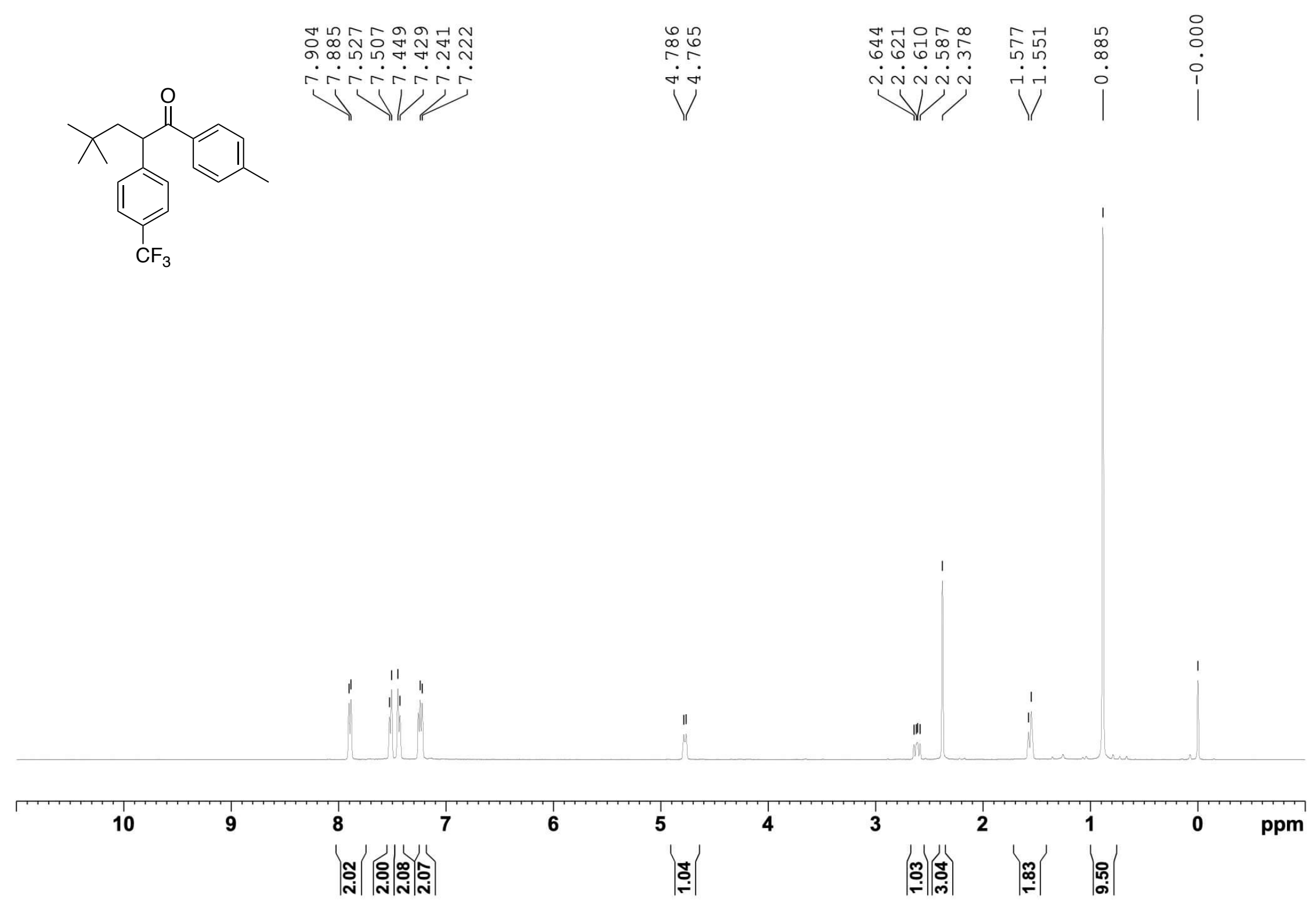




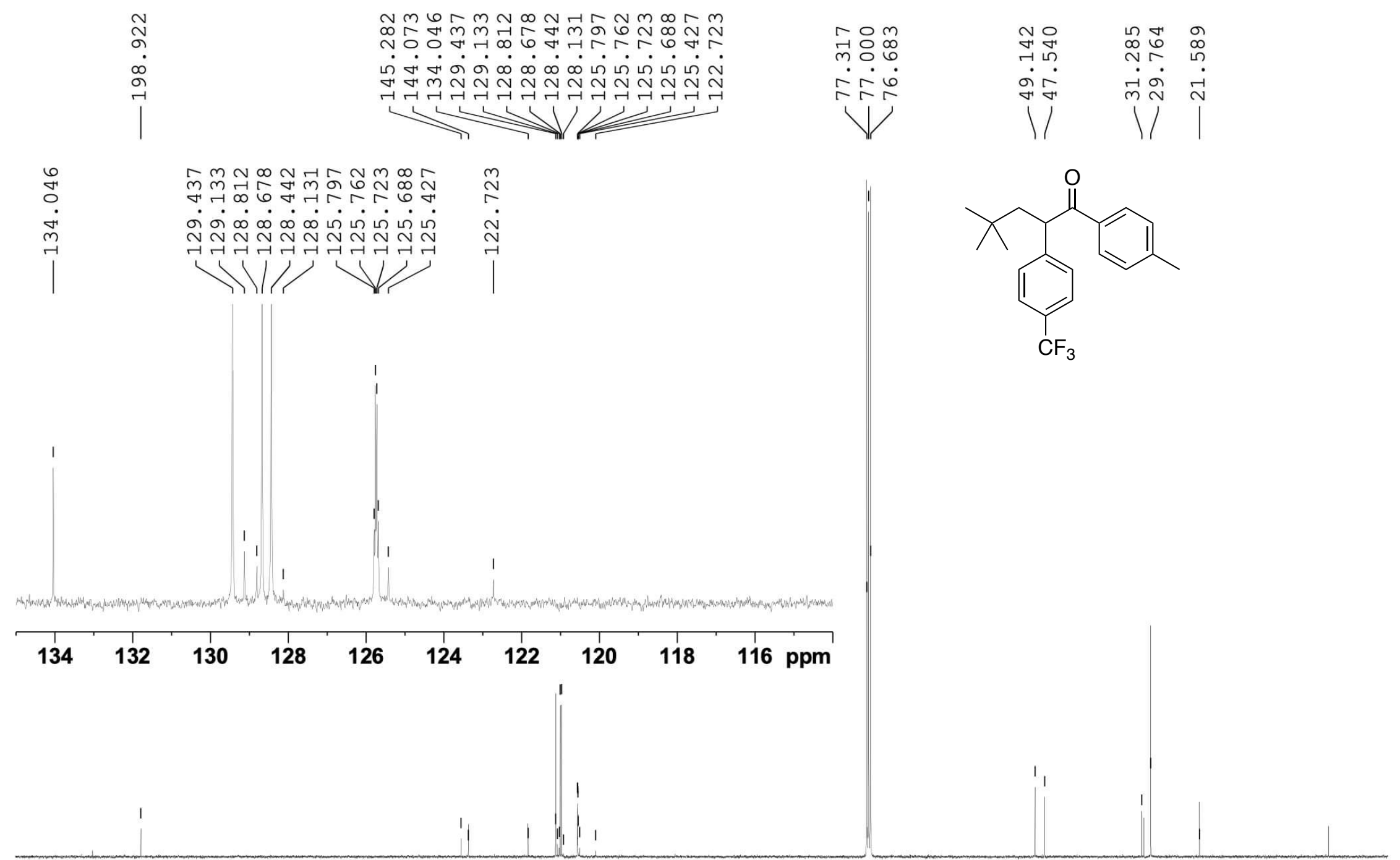

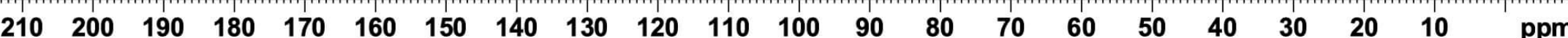


${ }^{19} \mathrm{~F}$ NMR of $\mathbf{6 a f a}$

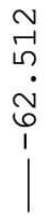
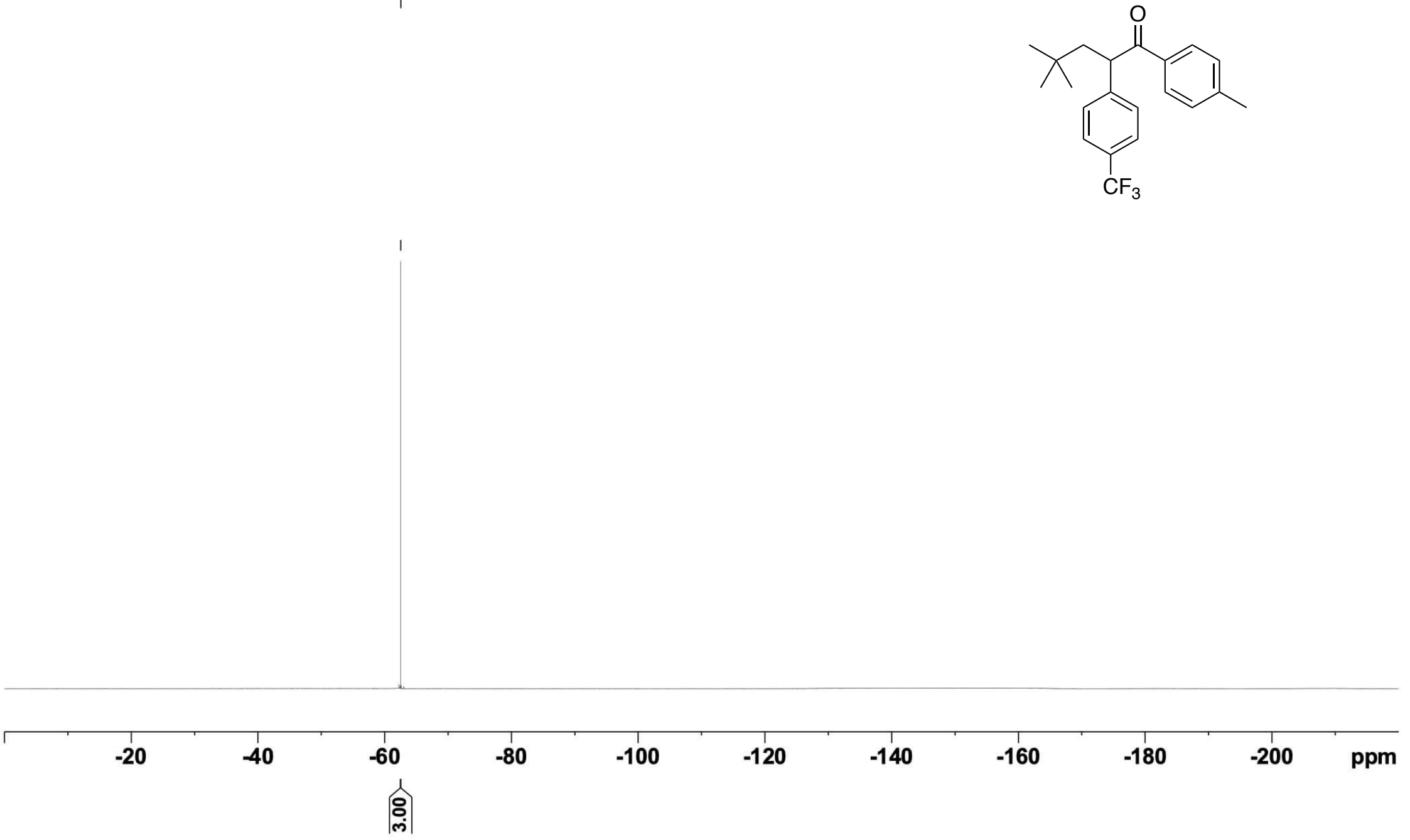


\section{${ }^{1} \mathrm{H}$ NMR of 6aga}

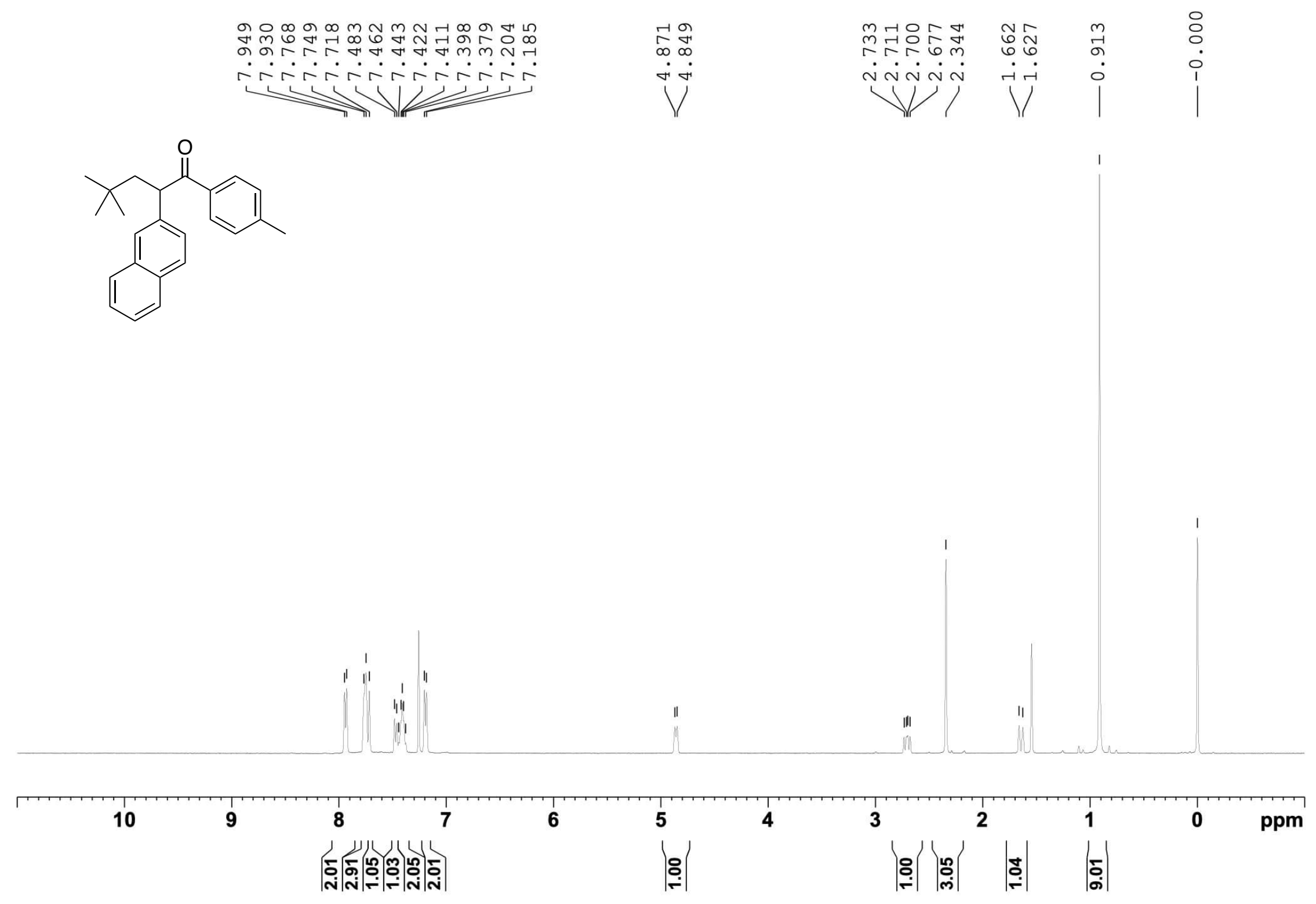




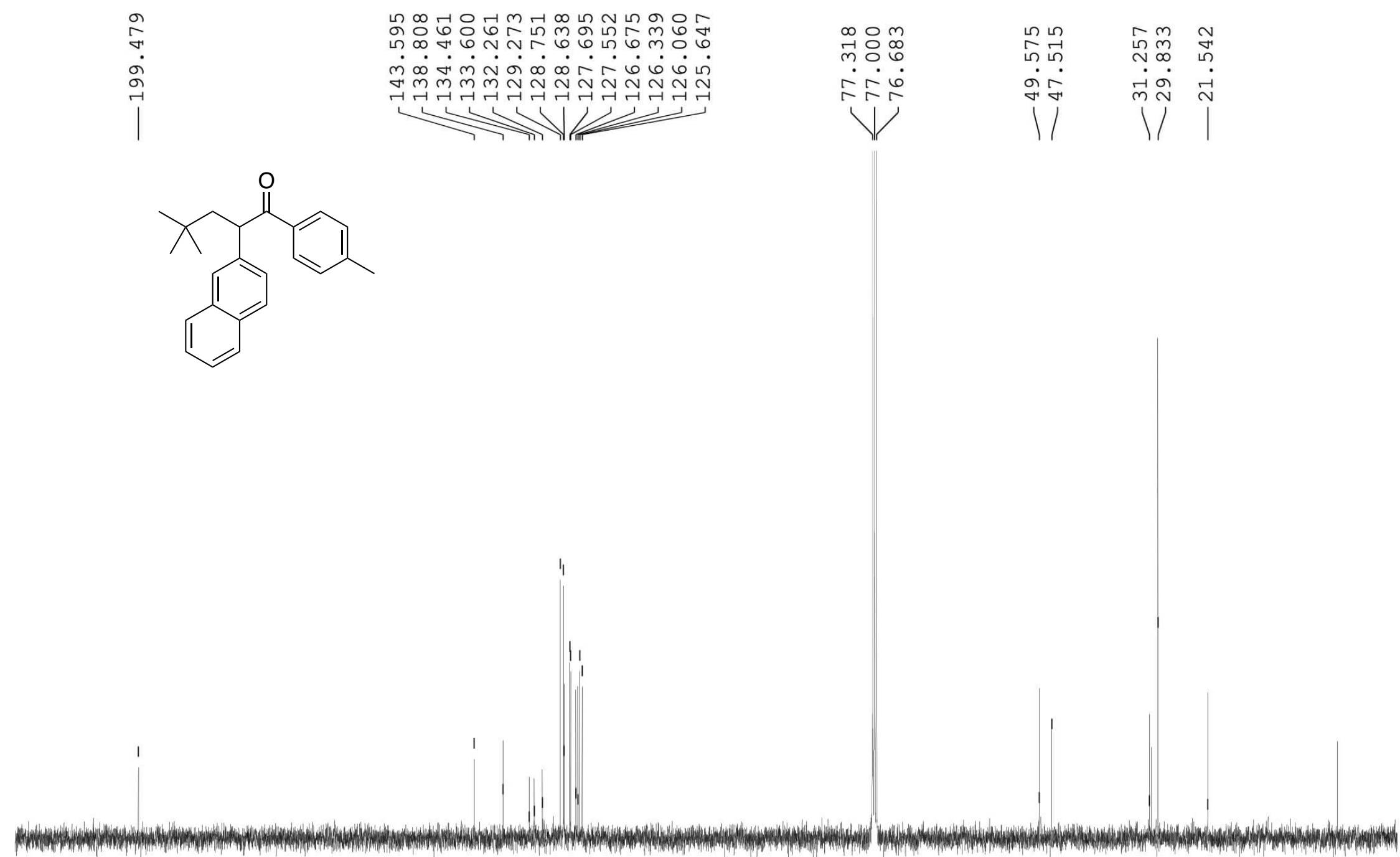

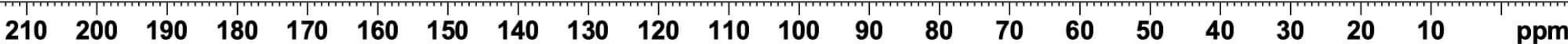



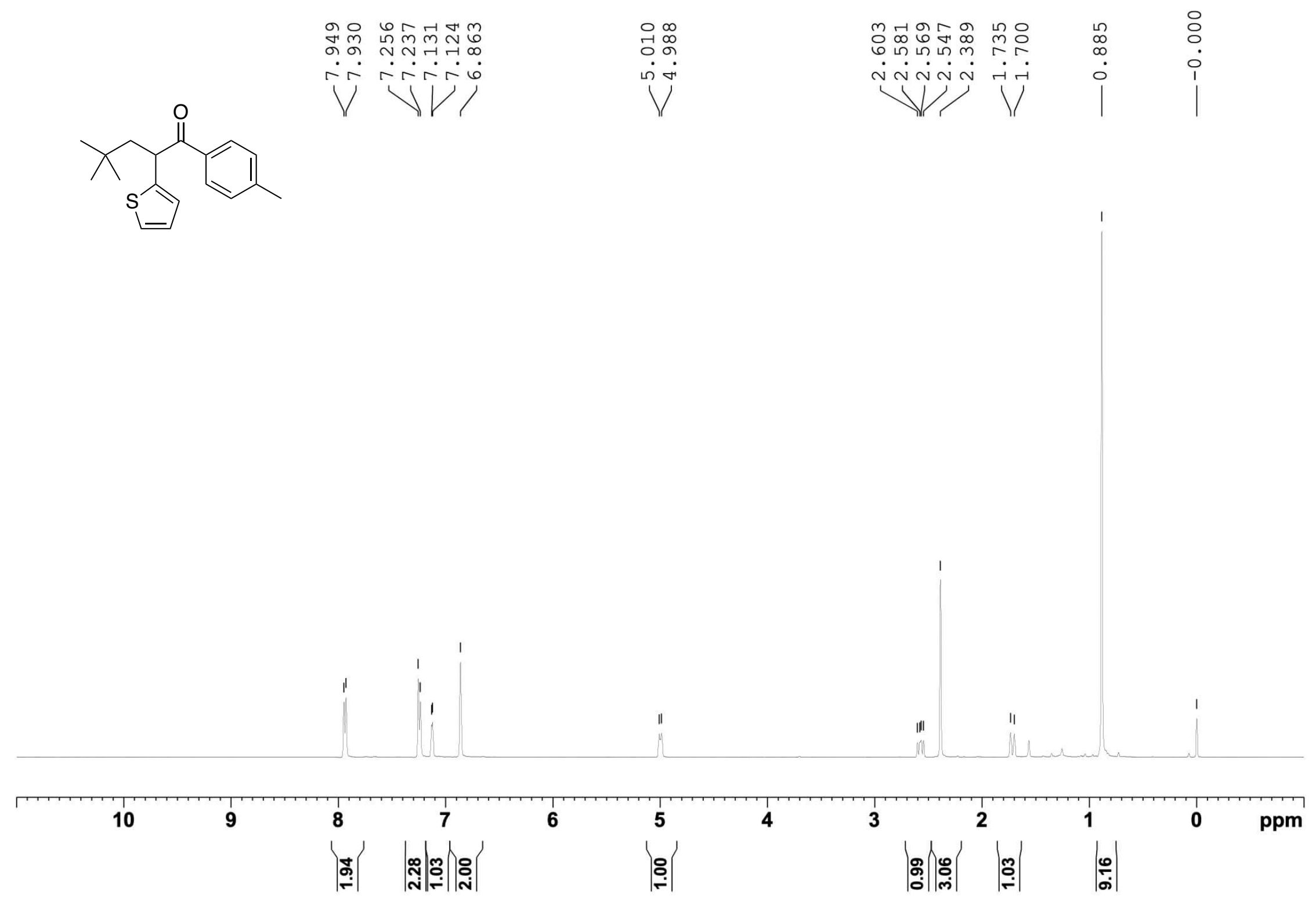
$m$
0
+
$\infty$
$\stackrel{0}{न}$
$\stackrel{1}{r}$

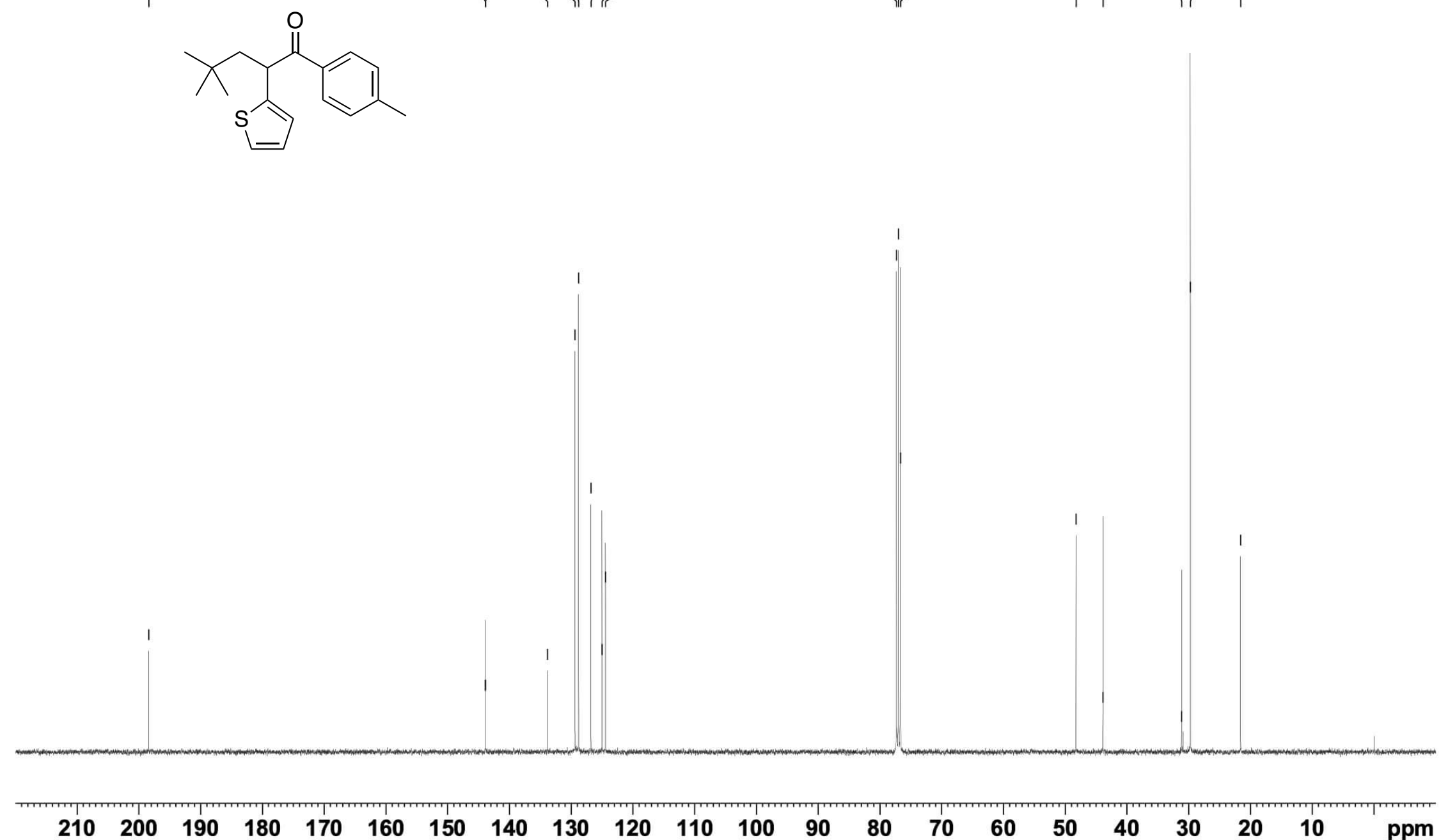

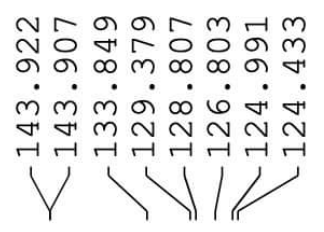

mim

둥

VI

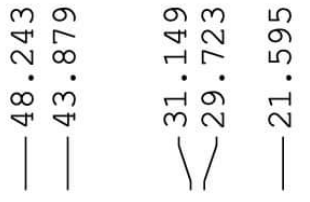



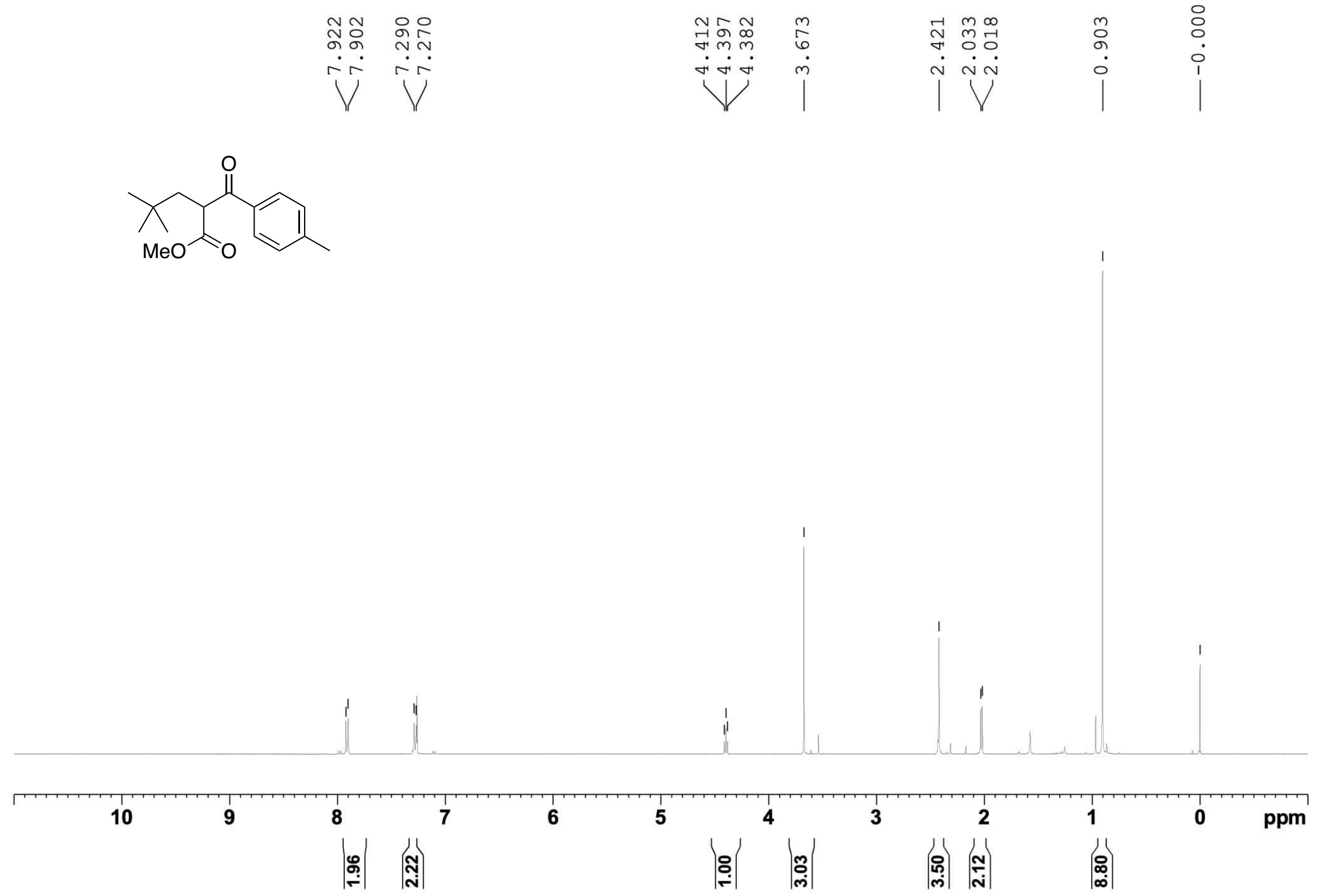

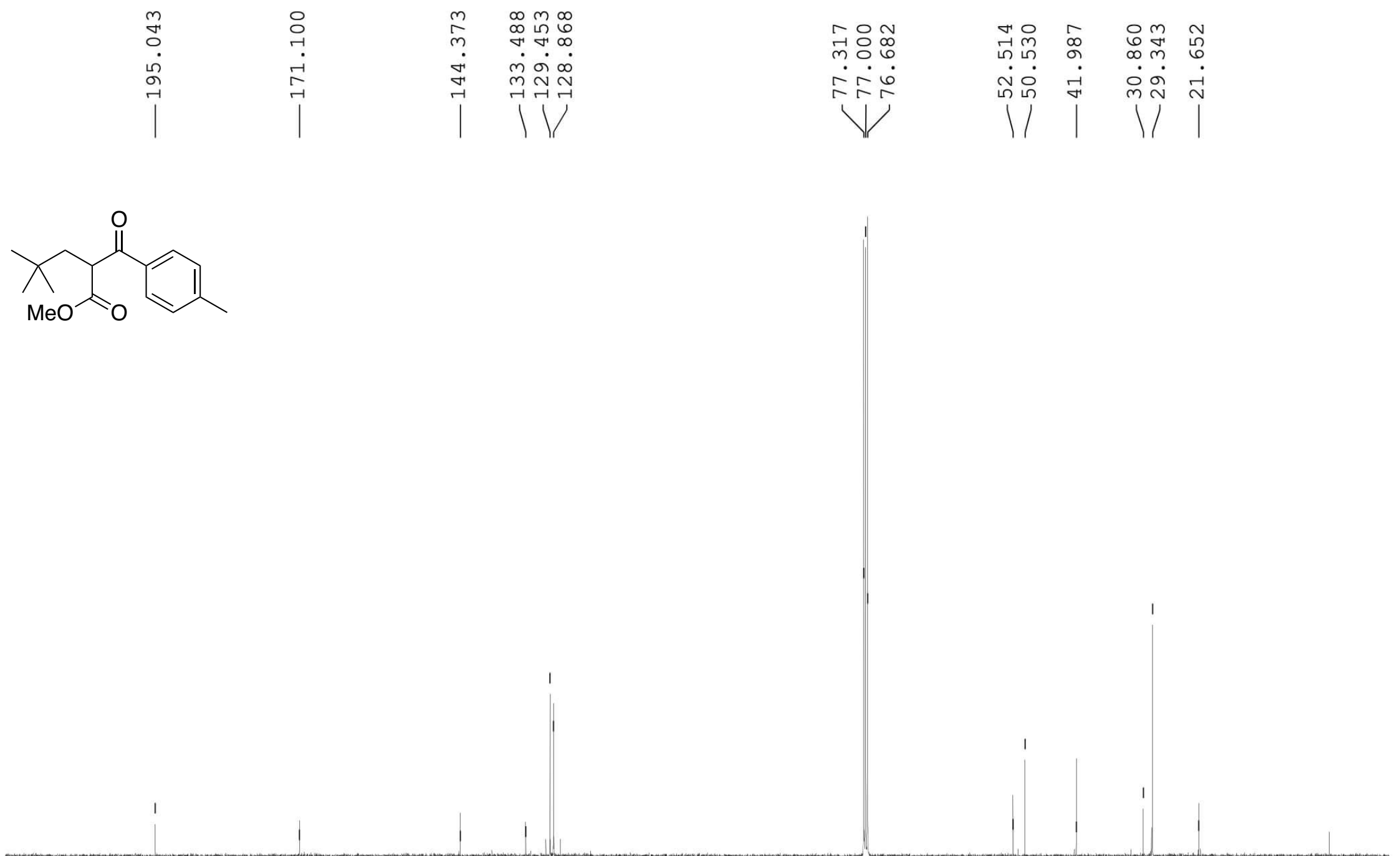

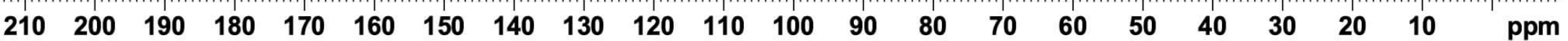

